Aus dem Institut für Ethik und Geschichte der Medizin

(Prof. Dr. C. Wiesemann)

im Zentrum Psychosoziale Medizin

der Medizinischen Fakultät der Universität Göttingen

\title{
Das Konzept der Vulnerabilität im Kontext transnationaler Biomedizin:
}

Eine ethische Analyse am Beispiel von Forschung mit Frauen in Indien

\author{
INAUGURAL - DISSERTATION \\ zur Erlangung des Doktorgrades \\ der Medizinischen Fakultät der \\ Georg-August-Universität zu Göttingen
}

\author{
vorgelegt von \\ Helen Grete Orth
}

aus

Neustadt an der Weinstraße

Göttingen 2014 
Dekan:

I. Berichterstatterin:

II. Berichterstatter:

III. Berichterstatter:

Tag der mündlichen Prüfung:
Prof. Dr. rer. nat. H. K. Kroemer

Prof. Dr. Silke Schicktanz (Betreurin)

Prof. Dr. Uwe Groß (Koreferent)

Prof. Dr. Martin Oppermann (Promotor)

19.06.2015 


\section{Inhaltsverzeichnis}

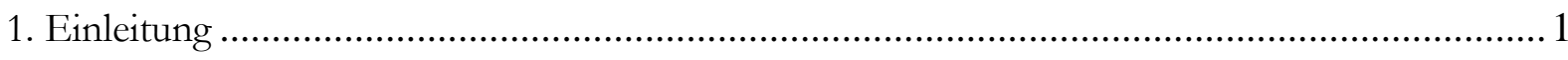

2. Methodisches Vorgehen und Aufbau der Arbeit ................................................................. 3

3. Ethisch-theoretische Kontextualisierung des Vulnerabilitätskonzepts..................................... 8

3.1 Was ist Vulnerabilität? - Verschiedene Definitionen......................................................... 8

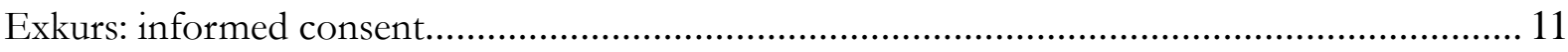

3.2 Vulnerabilität im Kontext medizinischer Forschung mit Frauen ...................................... 17

3.3 Vulnerabilität im Kontext medizinischer Forschung in Entwicklungsländern und der

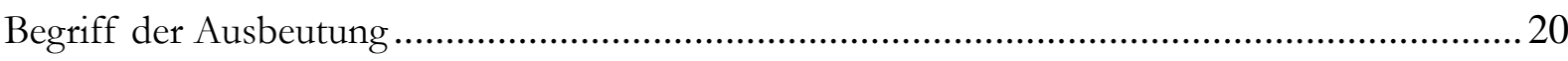

3.4 Vulnerabilitätskriterien für den Kontext medizinischer Forschung mit Frauen in

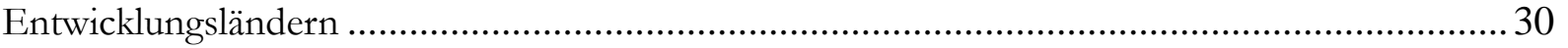

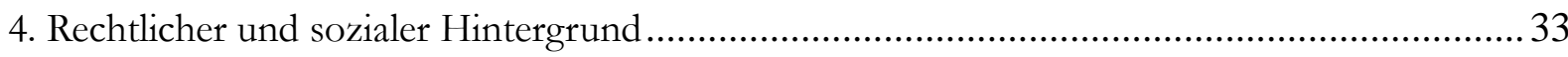

4.1 Vulnerabilität, medizinische Forschung mit Frauen und transnationale Biomedizin in

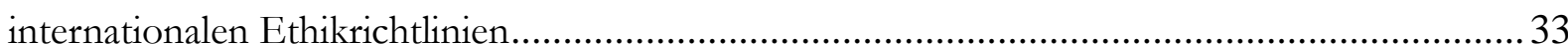

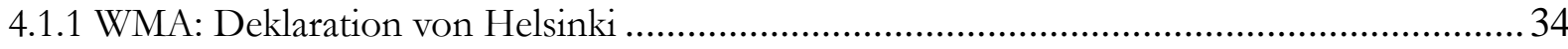

4.1.2 CIOMS: International Ethical Guidelines for Biomedical Research Involving Human

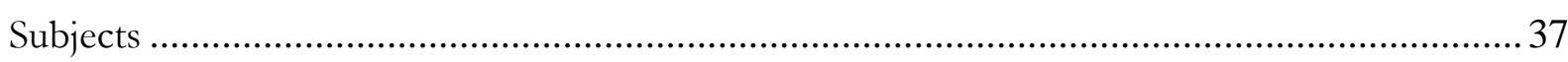

4.1.3 UNESCO: Universal Declaration on Bioethics and Human Rights ................................ 40

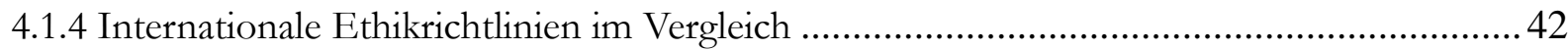

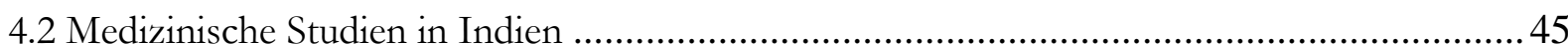

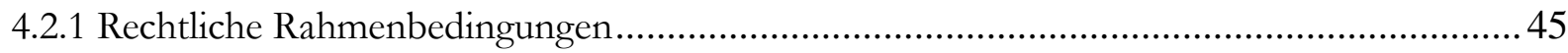

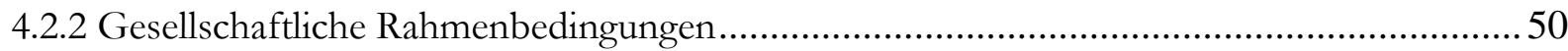


5. Analyse und Interpretation von zwei medizinischen Studien in Indien im Hinblick auf eine mögliche Vulnerabilität der Studienteilnehmerinnen

5.1 Fallstudie A: 'Safety and Immunogenicity Study of tgAAC09, a Gag-PR-RT AAV HIV

Vaccine'

5.1.1 Hintergrundinformationen und aktueller Forschungsstand zu Fallstudie A 61

5.1.2 Zielsetzung und Methodik der Fallstudie A 66

5.1.3 Bedingungen und StudienteilnehmerInnen der Fallstudie A 67

5.1.4 Verlauf und Ergebnisse der Fallstudie A 69

5.2 Interpretation der Fallstudie A im Hinblick auf Vulnerabilität der StudienteilnehmerInnen . 73

5.2.1 Ethische Aspekte bei der Durchführung von Fallstudie A 73

5.2.2 Vulnerabilität der StudienteilnehmerInnen in Fallstudie A 81

5.3 Fallstudie B: 'Evaluation of the Immune and Safety Response of GlaxoSmithKline (GSK) Biologicals' HPV Vaccine in Healthy Indian Women'..... 91

5.3.1 Hintergrundinformationen und aktueller Forschungsstand zu Fallstudie B 92

5.3.2 Zielsetzung und Methodik der Fallstudie B .98

5.3.3 Bedingungen und Studienteilnehmerinnen der Fallstudie B 100

5.3.4 Verlauf und Ergebnisse der Fallstudie B 101

5.4 Interpretation der Fallstudie B im Hinblick auf Vulnerabilität der Studienteilnehmerinnen 105

5.4.1 Ethische Aspekte bei der Durchführung von Fallstudie B 105

5.4.2 Vulnerabilität der Studienteilnehmerinnen in Fallstudie B 108

5.5 Kurzzusammenfassung und Vergleich der Ergebnisse beider Fallstudien 
5.6 Anwendung internationaler Ethikrichtlinien bei der ethischen Bewertung der.

Fallstudien

6. Diskussion und Fazit

6.1 Theoretische Implikationen für das Vulnerabilitätskonzept

6.1.1 Bedeutung der Ergebnisse für die Anwendung der erarbeiteten Vulnerabilitätskriterien .. 117

6.1.2 Vulnerabilität im Kontext transnationaler Biomedizin im Vergleich mit....

Vulnerabilität von Kindern

6.1.3 Kritik an der Praktikabilität und Notwendigkeit des Vulnerabilitätskonzepts

6.2 Praktische Implikationen für die Forschungsethik

6.2.1 Frauen in Indien als vulnerable Gruppe im Kontext transnationaler Biomedizin 126

6.2.2 Praktische Anwendung des Vulnerabilitätskonzepts: Schutzmaßnahmen versus Respekt für Autonomie

6.3 Ethische Konflikte im Kontext transnationaler Biomedizin: Ein Ausblick.....

7. Zusammenfassung

8. Anhang

8.1 Abbildungsverzeichnis 140

8.2 Tabellenverzeichnis

9. Literaturverzeichnis

10. Abkürzungsverzeichnis

Lebenslauf

Danksagung. 


\section{Einleitung}

Für Pharmafirmen aus Industriestaaten bringt das Verlegen medizinischer Forschung in Entwicklungsländer ${ }^{1}$, sogenannte transnationale Biomedizin, einige Vorteile mit sich (Mitra 2013). Diese sind unter anderem bis zu 60\% geringere Kosten für die Durchführung von Studien und eine schnellere Rekrutierung von ProbandInnen (Ballantyne 2010). Hierdurch verzeichnet transnationale Biomedizin in den letzten Jahren einen enormen Zuwachs (Ballantyne 2010). Transnationale Biomedizin birgt allerdings reichlich ethisches Konfliktpotenzial (Emanuel et al. 2004). Das wohl bekannteste Beispiel hierfür sind Placebo-kontrollierte HIV-Studien, die in den 90er Jahren mit Frauen in Entwicklungsländern (unter anderem in Uganda, Burkina Faso und Thailand) durchgeführt wurden (Lurie und Wolfe 1997). Ziel dieser Studien war es, ein geeignetes Medikament zur Verhinderung der HIV-Übertragung von Schwangeren auf deren Neugeborene zu finden. Probandinnen der Kontrollgruppe erhielten bei manchen dieser Studien ein Placebo, obwohl bereits ein Medikament existierte, das die Übertragung nachweislich senkt und in westlichen Ländern zu dieser Zeit bereits als Standard verwendet wurde. Mehr als 17.000 Frauen nahmen an diesen Studien teil, die vermutlich zu unzähligen verhinderbaren HIV-Infektionen bei Neugeborenen führten (Lurie und Wolfe 1997). Im Kontext transnationaler Biomedizin diskutieren viele AutorInnen, inwiefern ProbandInnen in Entwicklungsländern eine vulnerable Gruppe darstellen (Macklin 2003; Kottow 2003). Eine mögliche Vulnerabilität spezifisch von Frauen wird hingegen meist nur im Rahmen medizinischer Forschung mit Schwangeren erörtert (Wild 2007; Kopelman 2004, S. 2366f). Mit Blick auf die oben genannten HIV-Studien könnte es jedoch nicht unerheblich sein, dass diese gerade mit Frauen in Entwicklungsländern durchgeführt wurden. Da sozioökonomische Nachteile und patriarchalische Gesellschaftsstrukturen zu Menschenrechtsverletzungen von Frauen führen (Bunch 1990), stellt sich also zunächst die Frage, ob insbesondere Frauen im Kontext transnationaler Biomedizin bedingt durch soziale Faktoren eine vulnerable Gruppe darstellen könnten. In der Bioethik ist das Konzept der Vulnerabilität und dessen Anwendung allerdings nicht einheitlich definiert. Dies zeigt sich beispielsweise auch daran, dass Vulnerabilität zwar in wichtigen internationalen Ethikrichtlinien verwendet wird, jedoch jeweils mit einer unterschiedlichen Herangehensweise (Vgl. WMA: World Medical Association 2013a; CIOMS 2002). In dieser Arbeit soll nun untersucht werden, ob und inwiefern das Konzept der Vulnerabilität für die Betrachtung und normative Bewertung ethischer Aspekte im Kontext transnationaler Biomedizin mit Frauen angewendet werden kann. Dies soll exemplarisch anhand transnationaler Forschung mit Frauen in

\footnotetext{
${ }^{1}$ In dieser Arbeit gilt die Definition von Entwicklungsland des Bundesministeriums für wirtschaftliche Zusammenarbeit und Entwicklung und die Liste für Entwicklungsländer des Entwicklungsausschusses der OECD (DAC). Siehe hierzu: Bundesministerium für wirtschaftliche Zusammenarbeit und Entwicklung (2013); Entwicklungsausschuss der OECD (DAC) (2013)
} 
Indien geschehen. Für transnationale Biomedizin stellt Indien ein besonders beliebtes Ziel dar. Im Jahre 2011 waren über 1.800 Studien in Indien registriert (Pandey et al. 2011). Hierfür gibt es zahlreiche Gründe wie geringere Kosten, vergleichsweise hoher technischer Standard, Kommunikation auf Englisch und eine hohe genetische Diversität der Bevölkerung (Sengupta 2009). Allerdings gibt es auch hier einige Beispiele für unethische Vorgehensweisen (Sengupta 2009). Innerhalb von sieben Jahren (2005-2012) traten in 475 Studien 2.644 Todesfälle auf. Nur 3\% dieser Todesfälle wurden offiziell als mit der Studie in Zusammenhang stehend bewertet und hiervon erhielten wiederum nur die Hälfte der Familien eine Entschädigung (Jesani 2013). Die Veröffentlichung dieser Zahlen führte Anfang des Jahres 2013 zu einer Gesetzesänderung bezüglich klinischer Studien in Indien, die unter anderem die Entschädigung bei Verletzung oder Todesfällen von StudienteilnehmerInnen regelt und Ethikkomitees zur Registrierung verpflichtet (Jesani 2013).

Diese Arbeit soll einen wichtigen Beitrag zur Auseinandersetzung einer möglichen Vulnerabilität von Frauen im Kontext transnationaler Biomedizin aufgrund sozialer Aspekte, unabhängig von Schwangerschaft, leisten. Hierzu gilt es, exemplarisch die Situation von Frauen in Indien als Probandinnen genauer zu beleuchten. Demnach sollen Studien, die mit Frauen in Indien durchgeführt wurden, als Fallstudien mit Blick auf eine mögliche Vulnerabilität der Probandinnen analysiert werden. Diese empirisch orientierten Analysen erlauben eine ethisch-normative Bewertung bezüglich des Konzepts der Vulnerabilität und Schlussfolgerungen für dessen Anwendung für transnationale Biomedizin. Im nächsten Kapitel sollen nach der Erläuterung der zentralen Fragestellung die Methodik und der Aufbau der Arbeit vorgestellt werden. 


\section{Methodisches Vorgehen und Aufbau der Arbeit}

Diese Arbeit widmet sich der Frage, inwiefern das Konzept der Vulnerabilität für die Bearbeitung ethischer Aspekte im Kontext transnationaler Biomedizin sinnvoll anwendbar ist und dies insbesondere mit Blick auf Frauen in Indien als Studienteilnehmerinnen. Zu Beginn der Arbeit wird in Kapitel 3 Ethisch-theoretische Kontextualisierung des Vulnerabilitätskonzepts das Konzept der Vulnerabilität anhand verschiedener Definitionen und Herangehensweisen aus ethisch-theoretischer Sicht beleuchtet und in den Kontext transnationaler Biomedizin und Forschung mit Frauen gestellt. In Kapitel 4 Rechtlicher und sozialer Hintergrund folgt die Darlegung unterschiedlicher Herangehensweisen bezüglich Vulnerabilität, Forschung in Entwicklungsländern und Forschung mit Frauen in folgenden internationalen Ethikrichtlinien:

- Deklaration von Helsinki der World Medical Association (WMA)

- International Ethical Guidelines for Biomedical Research Involving Human Subjects des Council for International Organizations of Medical Sciences (CIOMS)

- Universal Declaration on Bioethics and Human Rights der United Nations Educational, Scientific and Cultural Organization (UNESCO)

Im Anschluss werden die Richtlinien bezüglich der oben genannten Themen miteinander verglichen. Des Weiteren beinhaltet Kapitel 4 theoretische Grundlagen hinsichtlich klinischer Studien in Indien. Diesbezüglich werden sowohl rechtliche als auch gesellschaftliche Rahmenbedingungen dargestellt, die ethische Aspekte im Kontext transnationaler Forschung in Indien beeinflussen können. Als Grundlage für diese Arbeit wurden sowohl im Vorfeld als auch im Laufe des Schreibprozesses zu den Themenschwerpunkten der jeweiligen Kapitel ausführliche Literaturrecherchen durchgeführt. Es handelt sich allerdings nicht um systematische Reviews, sondern vielmehr um eine Auswahl. Faktoren dabei sind zum einen die Aktualität der Literatur und zum anderen die jeweiligen Themenbereiche, wie beispielsweise Grundlagen zum Konzept der Vulnerabilität, ethische Konflikte im Rahmen von Forschung in Entwicklungsländern, internationale Ethikrichtlinien und Rahmenbedingungen von medizinischen Studien in Indien. Für die Literaturrecherchen wurden folgende Datenbanken verwendet:

- Pubmed: (http://www.ncbi.nlm.nih.gov/pubmed)

- Belit: (http://www.drze.de/belit/recherche/schnellsuche/recherche.html)

- Google Scholar: (http://scholar.google.de/) 
Folgende Schlüsselwörter und Kombinationen wurden sowohl auf Deutsch als auch auf Englisch hierbei angewendet: Vulnerabilität, vulnerable Gruppen, Entwicklungsländer, Indien, Frauen, klinische Studien/Forschung, Ethik, Bioethik, transnationale/internationale Biomedizin/Forschung, Ausbeutung, informed consent und Richtlinien.

Da die methodischen Vorgehensweisen von Kapitel 3 und 4 eine gänzlich theoretische Herangehensweise an das Konzept der Vulnerabilität verfolgen, soll im folgenden Abschnitt erläutert werden, warum bei der Bearbeitung bioethischer Fragestellungen auch empirische Mittel wichtig sein können und wie dies in dieser Arbeit umgesetzt wurde. In den letzten Jahren gibt es in der Medizinethik die Tendenz, vermehrt empirische Methoden anzuwenden (Schicktanz 2009). Dies bringt den Vorteil mit sich, dass eine erhöhte Kontextsensitivität und ein vermehrtes Sichtbarmachen von sozialen Rollen möglich werden. Zudem kann mithilfe empirischer Forschung in der Bioethik die Perspektive von Betroffenen verbessert abgebildet werden (Schicktanz 2009). Um die Auswirkungen ethischer Prinzipien in der Realität beurteilen zu können, benötigt die Medizinethik sozialwissenschaftliche Methoden (Birnbacher 1999). Das Konzept der Vulnerabilität ist in erster Linie ein ethischtheoretisches Konzept und es gibt bisher weder eine einheitliche Definition noch einen Konsens, ob und wie das Konzept in der Bioethik eingesetzt werden könnte. Somit soll in dieser Arbeit eine Brücke zwischen Theorie und Praxis geschlagen werden. Anhand von Fallstudien soll beleuchtet werden, inwiefern das Konzept der Vulnerabilität in der Praxis einsetzbar ist und dies insbesondere für den Kontext transnationaler Biomedizin mit Frauen. Folglich dienen die Fallstudien dazu, das Konzept der Vulnerabilität mit empirischen Mitteln zu prüfen und hierdurch eine erhöhte Kontextsensitivität herzustellen. Hierzu werden zunächst im theoretischen Teil dieser Arbeit mithilfe verschiedener Definitionen und Herangehensweisen spezifische Vulnerabilitätskriterien für den Kontext transnationale Forschung mit Frauen erarbeitet.

In Kapitel 5 Analyse und Interpretation von zwei medizinischen Studien in Indien im Hinblick auf eine mögliche Vulnerabilität der Studienteilnehmerinnen werden diese Vulnerabilitätskriterien auf die Fallstudien angewendet. Die analysierten Fallstudien sind zwei transnationale klinische Studien, die mit Frauen in Indien durchgeführt wurden. Bei der ersten handelt es sich um eine HIV-Impfstudie (Fallstudie A) und bei der zweiten um eine HPV-Impfstudie (Fallstudie B). Erstere wurde sowohl mit männlichen als auch weiblichen StudienteilnehmerInnen durchgeführt.

Für die Auswahl dieser beiden Fallstudien wurde im Zeitraum Mai 2012 bis Oktober 2012 mithilfe folgender Webseiten nach klinischen Studien recherchiert, die in Indien mit Frauen durchgeführt wurden:

- ClinicalTrials.gov: (http://clinicaltrials.gov/)

- The Cochrane Library: (http://www.thecochranelibrary.com/view/0/index.html)

- Clinical Trials Registry India (CTRI): (http://ctri.nic.in/Clinicaltrials/login.php) 
Bei ClincialTrials.gov ergaben sich im September 2012 anhand folgender Suchkriterien 209 mögliche Fallstudien:

- abgeschlossener Rekrutierungsstatus

- Studien mit Studienergebnissen

- Interventionsstudien

- Ort: Indien

- Geschlecht: weibliche Studienteilnehmer

- Alter: 18-65 Jahre

- Finanzierung durch Industrie

Mithilfe der Cochrane Librabry konnten anhand der Suchkriterien women und India im Abstract, Titel und Schlagwortverzeichnis 71 mögliche Fallstudien gefunden werden. Die Registrierungsplattform für medizinische Studien in Indien, CTRI, wurde im Oktober 2012 gezielt nach Studien durchsucht, die HIV als beforschtes Gesundheitsproblem angaben. Hierbei ergaben sich 38 Treffer. In der Vergangenheit wurden bereits unethische HIV-Studien in Entwicklungsländern durchgeführt (siehe hierzu Kapitel 1) und HIV stellt vor allem auch für Frauen in Indien ein großes Problem dar (siehe hierzu Kapitel 5.1.1). Darüber hinaus ist die Frage nach Vulnerabilität von Frauen im Kontext von HIV-Studien aufgrund gesellschaftlicher Aspekte, wie beispielsweise Stigmatisierung, besonders interessant. Aus diesen Gründen wurde bei CTRI gezielt nach HIV-Studien gesucht.

Bei der Auswahl der Fallstudien wurden aufgrund mangelhafter Informationslage Studien ausgeschlossen, die zum Zeitpunkt der Recherche weder abgeschlossen noch publiziert waren oder vorzeitig beendet wurden. Um eine mögliche Vulnerabilität von erwachsenen Frauen im Kontext transnationaler Biomedizin überprüfen zu können, wurden zudem Studien von der Auswahl ausgeschlossen, die mit Schwangeren, psychisch erkrankten Frauen oder ProbandInnen unter 18 Jahren durchgeführt wurden, da diese Personengruppen in der Bioethik bereits meist als vulnerable Gruppe bezeichnet werden. Außerdem galt eine Studienfinanzierung aus Indien als ein weiteres Ausschlusskriterium für eine Fallstudie, da hierdurch der Kontext transnationaler Biomedizin nicht gegeben ist. Die Schwierigkeit bei der Auswahl einer geeigneten Fallstudie lag darin, dass die Mehrzahl der registrierten Studien in vielen verschiedenen Ländern gleichzeitig durchgeführt wurden. Eine Zuordnung, wie viele Personen in Indien an der Studie teilnahmen oder welche Zusatzinformationen auf die indischen StudienteilnehmerInnen zutreffen, ist meist nicht veröffentlicht.

Nach Anwendung aller Auschlusskriterien kamen schließlich von insgesamt 318 Studien, die mithilfe der drei Suchportale gefunden wurden, nur fünf Studien tatsächlich als Fallstudien infrage. Die letztendliche Auswahl der HIV-Impfstudie als eine der Fallstudien begründet sich dadurch, dass in der 
dazugehörigen Publikation vergleichsweise viele und vor allem für die Analyse wichtige Informationen über die ProbandInnen, wie beispielsweise der Bildungsstatus, veröffentlicht wurden. Des Weiteren konnte speziell zu ethischen Aspekten bei der Planung und Durchführung der ausgewählten HIVImpfstudie Sekundärliteratur gefunden werden. Die Entscheidung, die HPV-Impfstudie als zweite Fallstudie auszuwählen, begründet sich unter anderem durch die bessere Vergleichbarkeit mit der ersten Fallstudie, da es sich bei beiden um Impfstudien handelt. Zudem stellt HPV bzw. Gebärmutterhalskrebs ein enormes Problem für die Gesundheit von Frauen in Indien dar (siehe hierzu Kapitel 5.3.1). Darüber hinaus wurden in Indien bereits HPV-Impfstudien durchgeführt, die aus ethischer Sicht als skandalös bezeichnet wurden (siehe hierzu Kapitel 5.3.1).

An dieser Stelle soll klargestellt werden, dass sich die Analyse und Interpretation der oben genannten Impfstudien auf Informationen gründen, die aus den jeweiligen Publikationen zu den Studien sowie aus Sekundärliteratur zur Verfügung standen. Folglich handelt es sich um eine retrospektive ethische Bewertung der Studien, da die Durchführung beider Studien abgeschlossen und die jeweiligen Ergebnisse bereits publiziert wurden. Eine retrospektive ethische Analyse von Studien ist insofern sinnvoll, da hierdurch bestimmte ethische Probleme nachträglich sichtbar gemacht werden. Die ethische Bewertung der in Kapitel 1 erwähnten HIV-Studien trugen erheblich zur Sensibiliserung von ethischen Konflikten im Kontext transnationaler Biomedizin bei und initiierten im Fachdiskurs zahlreiche Debatten zu diesem Thema (vgl. hierzu Lurie und Wolfe 1997).

Für die Analyse beider Fallstudien wurden zunächst Hintergrundinformationen und der aktuelle Forschungsstand bezüglich HPV- bzw. HIV-Impfungen recherchiert. Es folgt jeweils eine Beschreibung der Zielstellung und Methodik der Fallstudien. Die jeweiligen Ein- und Ausschlusskriterien für eine Studienteilnahme sowie Informationen über die StudienteilnehmerInnen und deren Lebenssituation werden ebenfalls dargelegt. Bezüglich der StudienteilnehmerInnen der HPV-Impfstudie sind im Gegensatz zur HIV-Impfstudie leider nur wenige Informationen zu deren Lebenssituation veröffentlicht. Daraufhin wurden sowohl die AutorInnen als auch das veröffentlichende Journal schriftlich per Email um mehr Informationen gebeten. Die Erstautorin der Studie stellte hierauf einen groben Überblick über den sozioökonomischen Status und Bildungsstand der Studienteilnehmerinnen zur Verfügung. Die Bitte um mehr Details und einige Rückfragen blieb jedoch unbeantwortet. Der letzte Abschnitt beinhaltet Erläuterungen zum Verlauf und zu den Ergebnissen der jeweiligen Fallstudie. Der Interpretationsteil der Fallstudien diskutiert zunächst offene Fragen bezüglich ethischer Aspekte der Studiendurchführung. Hier werden Diskussionen über die Wirksamkeit der jeweiligen Impfungen aufgegriffen. Im Anschluss werden die in Kapitel 3 erarbeiteten Vulnerabilitätskriterien mithilfe der in den Analysen gewonnen Informationen auf die Fallstudien angewendet. Darauf folgt eine normative Bewertung, ob die StudienteilnehmerInnen im Rahmen ihrer Studienteilnahme 
vulnerabel waren. Nach einem Zwischenfazit wird diskutiert, inwiefern die in Kapitel 4 vorgestellten internationalen Ethikrichtlinien hilfreich bei der Analyse und Interpretation der Fallstudien waren.

In Kapitel 6 Diskussion und Farit wird anhand der Ergebnisse der Fallstudien die Anwendung des eigens entwickelten Vulnerabilitätskonzepts diskutiert und erläutert, inwiefern es praxisrelevant ist. Hinsichtlich theoretischer Implikationen für das Vulnerabilitätskonzept werden unter anderem mögliche Vulnerabilitätskriterien von Kindern, die üblicherweise als vulnerable Gruppe gelten, mit den in dieser Arbeit entwickelten Vulnerabilitätskriterien verglichen. Damit soll die Frage beantwortet werden, ob die verwendeten Vulnerabilitätskriterien universal für die Identifizierung und den Schutz von vulnerablen Personen oder Gruppen einsetzbar wären. Zudem werden verschiedene Kritikpunkte und die Notwendigkeit des Vulnerabilitätskonzepts für die Forschungsethik im Allgemeinen diskutiert. Im Zuge der Diskussion über eine mögliche praktische Umsetzung des Vulnerabilitätskonzepts werden die Ergebnisse der Fallstudien unter anderem mit Blick auf zukünftige medizinische Forschung mit Frauen in Indien erörtert. Zusätzlich wird diskutiert, inwiefern mögliche Schutzmaßnahmen zur Verhinderung von Vulnerabilität die Autonomie von StudienteilnehmerInnen einschränken könnten. Daraufhin werden den Vulnerabilitätskriterien jeweils konkrete Schutzmaßnahmen zugeordnet. Im abschließenden Ausblick werden verschiedene Vorschläge unterbreitet, deren Umsetzung zukünftig Vulnerabilität von ProbanInnen im Kontext transnationaler Biomedizin minimieren beziehungsweise bereits vor Beginn einer Studie verhindern soll, dass Vulnerabilität entsteht. 


\section{$\underline{\text { 3. Ethisch-theoretische Kontextualisierung des Vulnerabilitätskonzepts }}$}

\section{$\underline{3.1 \text { Was ist Vulnerabilität? - Verschiedene Definitionen }}$}

Das Konzept der Vulnerabilität wird in der Bioethik meist dann verwendet, wenn Kinder oder psychisch Kranke an medizinischen Studien teilnehmen. Trotzdem ist das Konzept an sich wenig untersucht (Levine et al. 2004). Dies zeigt sich beispielsweise dadurch, dass es in der Bioethik keine einheitlich verwendete Definition von Vulnerabilität gibt (DeBruin 2004; Hurst 2008).

Das Wort Vulnerabilität stammt von vulnerabilis (lateinisch) und bedeutet verwundbar oder verletzlich (Brockhaus Enzyklopädie, 21. Auflage, S. 282). Aufgrund der wörtlichen Bedeutung würde man somit Personen als vulnerabel bezeichnen, die verletzlich oder verwundbar sind. Dies trifft jedoch im weitesten Sinne auf alle Menschen zu. Folglich lässt sich nicht sofort erklären, warum gerade Kinder oder psychisch Kranke im Kontext medizinischer Forschung häufig als vulnerabel bezeichnet werden. In der Bioethik besteht der Konsens, dass vulnerable Individuen oder Personengruppen im Kontext medizinischer Forschung besondere Beachtung oder besonderen Schutz benötigen (Hurst 2008).

Zunächst sollen einige in der Diskussion befindliche Definitionen von Vulnerabilität genauer beleuchtet werden, um sinnvolle Kriterien für Vulnerabilität für den spezifischen Kontext dieser Arbeit aufzustellen. Die verschiedenen Definitionen werden in dieser Arbeit in kontextbezogene und personenbezogene Herangehensweisen eingeteilt. Kontextbezogen soll hier bedeuten, dass der Kontext der jeweiligen Studie bestimmt, ob und inwiefern ProbandInnen als vulnerabel gelten. Der Kontext der Studie beinhaltet Einflussfaktoren, die von der Studie selbst oder vom Forscherteam ausgehen. Hierzu zählt unter anderem die Konzipierung der Studie, zum Beispiel das Risiko, das mit einer Studienteilnahme einhergeht oder auch ein Vorenthalten relevanter Informationen von Seiten des Forscherteams. Personenbezogene Herangehensweisen stellen bestimmte Eigenschaften einer Person oder Personengruppe in den Vordergrund. In dieser Arbeit sollen alle Aspekte, die eine mögliche Vulnerabilität im Rahmen einer Studienteilnahme von Seiten der ProbandInnen beeinflussen als personenbezogene Aspekte zusammengefasst werden. Hierzu zählen sowohl Eigenschaften als auch der Kontext im Sinne der aktuellen Lebenssituation dieser Person oder Personengruppe. Solche Aspekte können zum Beispiel sowohl kognitive Fähigkeiten als auch die sozioökonomische Situation von Personen sein.

Hurst beleuchtet verschiedene Definitionen von Vulnerabilität und kommt jeweils zu der Schlussfolgerung, dass diese entweder zu weit oder zu eng gefasst seien. Um dies zu verhindern, schlägt sie folgende Herangehensweise vor: „I propose that vulnerability as a claim to special protection should be understood as an identifiably increased likelihood of incurring additional or greater wrong” (Hurst 
2008, S. 195). Der Fokus müsse hierbei auf den Gegebenheiten der Studie und des Umfeldes liegen, anstatt auf den Eigenschaften möglicher ProbandInnen, fordert Hurst (Hurst 2008). Folglich beschreibt sie eine in erster Linie kontextbezogene Definition. Als Beispiel für ein mögliches Unrecht im Kontext einer Studienteilnahme nennt sie ein unausgewogenes Nutzen-Risiko-Verhältnis. Hursts Herangehensweise bietet eine wichtige Grundlage zum Verständnis von Vulnerabilität, denn Personen, die mit einer erhöhten Wahrscheinlichkeit zusätzliches oder erhebliches Unrecht erleiden, haben ein Anrecht auf besonderen Schutz und können somit als vulnerabel bezeichnet werden. Um Aussagen über eine mögliche Vulnerabilität von Frauen in Indien im Kontext transnationaler Biomedizin machen zu können, ist die Definition von Hurst jedoch zu abstrakt. Es wäre beispielsweise schwierig zu klären, ob Frauen in Indien, die an transnationalen medizinischen Studien teilnehmen, eine erhöhte Wahrscheinlichkeit aufweisen, Unrecht zu erfahren. Hierbei müsste nämlich zunächst erläutert werden, wie sich eine erhöhte Wahrscheinlichkeit, Unrecht zu erfahren, auszeichnet und wer dies festlegt. Hurst schreibt selbst, dass diese Definition nicht frei sei von subjektiven Beurteilungen und Bewertungen und in einer Grauzone zu Unstimmigkeiten führen könnte (Hurst 2008). Meist sei jedoch die Grenze zwischen erhöhter und nicht erhöhter Wahrscheinlichkeit, Unrecht zu erfahren, ausreichend gekennzeichnet (Hurst 2008). Darüber hinaus gibt es Gründe, bestimmte Eigenschaften von ProbandInnen in die Definition von Vulnerabilität miteinzubeziehen, was Hurst jedoch nicht berücksichtigt. Die Rechte von PatientInnen, die im Koma liegen und als potenzielle ProbandInnen für eine medizinische Studie in Frage kommen, müssten beispielsweise besonders geschützt werden. Bedingt durch die momentane Eigenschaft, nicht kommunizieren zu können, können sich diese PatientInnen nämlich nicht selbst schützen. Dies würde bedeuten, sie sind vulnerabel. Die Konzipierung einer Studie könnte diese PatientInnen allerdings kontextabhängig mehr oder weniger vulnerabel machen. Eine Studie, die beispielsweise mit einem hohen Risiko einhergeht, Schaden zu erleiden, würde zu einem höheren Maß an Vulnerabilitat führen als eine Studie, die mit einem minimalen Risiko einhergeht. Die Eigenschaft dieser PatientInnen, nicht kommunizieren zu können, bleibt jedoch die Hauptursache für deren Vulnerabilität. Deswegen ist es sinnvoll, eine verstärkt kontextbezogene Definition von Hurst mit einer personenbezogenen Herangehensweise an das Konzept der Vulnerabilität zu ergänzen. Zudem wird die Definition von Hurst durch zusätzliche Kriterien intersubjektiv nachvollziehbarer und somit im Einzelfall besser anwendbar sein.

Eine mögliche personenbezogene Herangehensweise zur Ergänzung des Konzepts von Vulnerabilität, wird von Kottow dargestellt. Kottow versteht Vulnerabilität als eine Eigenschaft, die alle Menschen haben: „,...] vulnerability as well as dignity and integrity - are descriptive characteristics of human beings qua humans, which are not normative in themselves [...]“ (Kottow 2003, S. 281). 
Kottow betont, dass man zwischen Vulnerabilität und Anfälligkeit unterscheiden müsse:

„The distinction between vulnerability and susceptibility also marks the difference between being intact but fragile - vulnerable - and being injured and predisposed to compound additional harm susceptible“ (Kottow 2003, S. 460).

Anfällige Individuen seien biologisch geschwächt oder erkrankt und deswegen prädisponiert, zusätzliches Leid zu erfahren. Kottow erläutert, dass Anfälligkeit durch besondere und gezielt ausgerichtete Schutzmaßnahmen reduziert werden könne, wobei Vulnerabilität als Verletzbarkeit, die allen Menschen innewohnt, durch Maßnahmen des Staates in Form von Gesetzgebungen reduziert würde (Kottow 2003). Kottow verwendet hier den Begriff Anfälligkeit, um zu beschreiben, was in der Bioethik üblicherweise als Vulnerabilität bezeichnet wird. Kottow macht diese begriffliche Unterscheidung, um zu verhindern, dass ForscherInnen anfällige Individuen als vulnerabel bezeichnen und sich somit der Verantwortung entziehen, diese verstärkt schützen zu müssen (Kottow 2003). Würden anfällige Individuen, laut Kottow, fälschlicherweise als vulnerabel gelten, bliebe die Aufgabe zur Reduzierung der Vulnerabilität nämlich dem Staat und nicht dem Forscherteam zugesprochen.

Da das Konzept der Vulnerabilität in der Bioethik häufig verwendet wird, ist es jedoch sinnvoll, den Begriff Vulnerabilität beizubehalten. Eine Änderung der Begriffe von Vulnerabilität zu Anfälligkeit, wie Kottow es fordert, könnte unnötige Verwirrung schaffen und die Aussagekraft des Konzepts der Vulnerabilität vermindern, obwohl dieses in der Bioethik ein etabliertes Konzept darstellt. Der Begriff Anfälligkeit suggeriert vielmehr eine körperliche Schwäche. Eine Sensibilisierung für Personen, die zum Beispiel aufgrund ihrer sozioökonomischen Situation besonders verletzbar sind, könnte hierdurch erschwert werden. An dieser Stelle ist es somit wichtig klarzustellen, dass Vulnerabilität, trotz der Anerkennung als eine Eigenschaft, die alle Menschen teilen, in dieser Arbeit ein spezifisches Konzept bezeichnet, das Individuen oder Personengruppen identifizieren kann, die in bestimmten Kontexten besonderen Schutz benötigen. Kottows Verständnis von Anfälligkeit, nämlich die Prädisposition für zusätzliches Leid, soll in dieser Arbeit eine personenbezogene Ergänzung zu Hursts Ansatz von Vulnerabilität sein. Da Kottow jedoch einen kontextbezogenen Ansatz ausblendet, bleibt Hursts Definition als Grundlage notwendig. Denn Individuen, die anfällig sind für zusätzliches Leid, benötigen besonderen Schutz. Allerdings schreibt Kottow nur von zusätə̧lichem Leid. In diesem Fall würden zum Beispiel Kinder nicht als vulnerabel gelten, wenn sie kein zusätzliches, sondern erstmaliges Leid erfahren würden. Es müssten jedoch auch Individuen, die eine erhöhte Prädisposition aufweisen, überhaupt Leid zu erfahren, besonders geschützt werden. Die Prädisposition, zusätzliches Leid zu erfahren, reicht daher als Kriterium für Vulnerabilität als personenbezogene Herangehensweise nicht aus, um vulnerable Personen von nicht vulnerablen Personen zu unterscheiden. Ein weiteres mögliches Kriterium für Vulnerabilität, das beispielsweise auf Kinder zutreffen könnte und unabhängig von zusätzlichem oder erstmaligem Leid geprüft werden könnte, ist die Unfähigkeit, eine informierte 
Zustimmung (engl. informed consent) zu geben. Eine Definition von Vulnerabilität, die den informed consent in den Fokus rückt, könnte somit hilfreich sein, um Kottows Herangehensweise sinnvoll zu ergänzen. Um die Bedeutung des informed consent für Vulnerabilität zu erörtern, soll zunächst das Konzept des informed consent genauer erläutert werden.

\section{Exkurs: informed consent}

Da Principles of Biomedical Ethics von Beauchamp und Childress eines der einflussreichsten Medizinethikbücher darstellt, dient dieses als Grundlage für die Erläuterungen des informed consent (Schöne-Seifert 2005, S. 701). Alle nennenswerten Bioethikrichtlinien, wie zum Beispiel die Deklaration von Helsinki, fordern, dass vor einer medizinischen Intervention oder Studienteilnahme der informed consent der PatientInnen/ProbandInnen eingeholt werden muss (Beauchamp und Childress 2013, S. 121). Da die Vulnerabilität von StudienteilnehmerInnen im Mittelpunkt dieser Arbeit steht, soll die Bedeutung des informed consent im Hinblick auf medizinische Studien erläutert werden. Informed consent bedeutet die selbstbestimmte Einwilligung in eine Studienteilnahme und dient dem Schutz der Autonomie der ProbandInnen (Beauchamp und Childress 2013, S. 121). Beauchamp und Childress nennen sieben dem informed consent zugrundeliegende Elemente. Diese lauten:

1. Kompetenz (zu verstehen und zu entscheiden)

2. Freiwilligkeit (der Entscheidung)

3. Aufklärung (Offenlegen relevanter Information)

4. (Empfehlung)

5. Verstehen (von Punkt 3 und 4)

6. Entscheidungsfindung

7. Zustimmung (Beauchamp und Childress 2013, S. 124f)

Kompetenz stellt in erster Linie eine Voraussetzung für die darauffolgenden Elemente dar (Beauchamp und Childress 2013, S. 124). Die Ermittlung der Kompetenz von ProbandInnen bezieht sich auf die psychologische oder juristische Fähigkeit, eine adäquate Entscheidung treffen zu können (Beauchamp und Childress 2013, S. 114). Durch die Beurteilung der Kompetenz wird unterschieden zwischen Personen, deren Entscheidungen akzeptiert werden sollten, und Personen, deren Entscheidungen nicht akzeptiert werden müssen. Die Kompetenz einer Person kann variieren und steht jeweils in Bezug zu der zu treffenden Entscheidung (Beauchamp und Childress 2013, S. 114f). Eine Person könnte zum Beispiel entscheidungsfähig sein, wenn es sich um die Zustimmung für eine Blutentnahme handelt, jedoch gleichzeitig nicht fähig sein, die Entscheidung für oder wider eine Chemotherapie zu treffen. Es wäre möglich, dass diese Person, beispielsweise aufgrund eines niedrigen Bildungsstands, die medizinische Notwendigkeit im Verhältnis zu den damit verbunden Risiken im Falle einer Blutentnahme, nicht jedoch im Falle einer Chemotherapie abwägen kann, da diese komplexer und 
schwerwiegender sind. Im Kontext medizinischer Forschung wird eine Person üblicherweise als kompetent bezeichnet, wenn sie fähig ist, studienrelevante Informationen $\mathrm{zu}$ verstehen, sie zu beurteilen, über die Hauptrisiken und -nutzen zu reflektieren und daraufhin eine Entscheidung zu treffen (Beauchamp und Childress 2013, S. 117).

Freiwilligkeit bedeutet, eine Person beabsichtigt eine Handlung, ohne dabei unter kontrollierendem Einfluss zu stehen. Hierbei steht bei Beauchamp und Childress externe Beeinflussung durch andere Individuen im Vordergrund (Beauchamp und Childress 2013, S. 138). Nicht jegliche Form der Beeinflussung durch andere sei gleichzeitig kontrollierend. Entscheiden sich beispielsweise PatientInnen aufgrund der Beratung durch behandelnde ÄrztInnen für eine bestimmte Therapiemöglichkeit, obwohl sie dieser ursprünglich skeptisch gegenüber standen, sprechen Beauchamp und Childress zwar von Beeinflussung, jedoch nicht von kontrollierendem Einfluss. Hätten die PatientInnen jedoch keine andere Wahl als der angebotenen Therapieoption zuzustimmen, da ÄrztInnen sonst weitere Behandlung verweigern, handele es sich um kontrollierenden Einfluss (Beauchamp und Childress 2013, S. 138).

Das dritte Element, die Aufklärung, soll als Grundlage für die Entscheidungsfindung dienen (Beauchamp und Childress 2013, S. 125). Beauchamp und Childress zählen hierzu Informationen, die ForscherInnen als wesentlich erachten, die Empfehlung der ForscherInnen, das Ziel der Zustimmung und die Bedeutung und Grenzen des informed consent als Akt der Einwilligung. Zusätzlich sollten Ziele und Methoden der Studie, Nutzen und Risiken sowie begleitende Unannehmlichkeiten erläutert werden. Hinzu kommt die Aufklärung der ProbandInnen über ihr Recht, die Zustimmung ohne dadurch zu erwartende Nachteile jederzeit widerrufen zu können (Beauchamp und Childress 2013, S. 125).

Das vierte Element Empfehlung ist im Rahmen des informed consent-Prozesses bei medizinischen Studien nicht zwingend notwendig und wird deswegen in dieser Arbeit nicht genauer erläutert (Beauchamp und Childress 2013, S. 124).

Das fünfte Element des informed consent ist das Verstehen relevanter Informationen. Laut Beauchamp und Childress gibt es keinen Konsens darüber, was Verstehen genau bedeutet. Sie schlagen vor, dass eine Person dann versteht, wenn sie sich sachdienliche Informationen angeeignet hat und eine konkrete Vorstellung von der Art und Tragweite ihrer Handlungen hat (Beauchamp und Childress 2013, S. 131). ProbandInnen sollten zumindest die Informationen verstehen, die gemäß sorgfältiger ForscherInnen verstanden werden müssten, um einer Studienteilnahme zustimmen zu können (Beauchamp und Childress 2013, S. 132). Hierzu zählen Diagnose und Prognose im Fall erkrankter ProbandInnen, das Ziel einer medizinischen Intervention, Alternativen, Risiken und Nutzen sowie eine Empfehlung der ForscherInnen, wobei letzteres im Kontext medizinischer Studien nicht unbedingt notwendig sei (Beauchamp und Childress 2013, S. 132). Beauchamp und Childress erläutern hierzu, dass Forderungen 
nach vollkommener Aufklärung und völligem Verstehen zu einem falschen Ideal führen würden, denn es sei ausreichend, wenn relevante Informationen verstanden würden (Beauchamp und Childress 2013, S. 132). Beauchamp und Childress ergänzen, dass der Begriff des informed consent auch stets die Möglichkeit des informed refusal, nämlich die Ablehnung einer medizinischen Intervention oder Studienteilnahme, beinhalten müsse.

\section{Vulnerabilität und informed consent}

Der informed consent spielt eine wichtige Rolle bei der Prüfung verschiedener Definitionsmöglichkeiten von Vulnerabilität. International anerkannte Richtlinien in der Bioethik, wie zum Beispiel die Deklaration von Helsinki aus dem Jahre 2008 (aktuell 2013) und die Richtlinien des CIOMS verknüpfen Vulnerabilität mit informed consent (siehe hierzu Kapitel 4.1).

Zusätzlich wird in der Encyclopedia of Bioethics, einem wichtigen Nachschlagewerk in der Bioethik, Vulnerabilität mithilfe von informed consent erläutert. Zum einen gelten hier Personen als vulnerabel, die nicht fähig sind, informed consent zu erteilen, weil sie vom Schutz anderer abhängig seien. Hierzu würden zum Beispiel Kinder und Erwachsene zählen, die durch ein Trauma, eine Erkrankung, durch geistige Behinderung oder Demenz beeinträchtigt sind (Kopelman 2004, S. 2365). Zum anderen seien Personen vulnerabel, die mit einer erhöhten Wahrscheinlichkeit unter Zwang stehen oder manipuliert werden. Gefängnisinsassen, StudentInnen und Krankenhauspersonal werden hier als Beispiele genannt (Kopelman 2004, S. 2365). Darüber hinaus werden Personen als vulnerabel benannt, die aufgrund einer prekären finanziellen Situation versucht sein könnten, einer Studienteilnahme zuzustimmen, die sie unter anderen Umständen abgelehnt hätten. Folglich zeigt sich in der Encyclopedia of Bioethics jeweils eine direkte oder indirekte Verknüpfung von Vulnerabilität und informed consent. Denn eine zweifelhafte Freiwilligkeit von StudienteilnehmerInnen aufgrund von Zwang oder Manipulation stellt letztlich die ethische Gültigkeit des informed consent infrage und verbindet somit Vulnerabilität indirekt mit informed consent. Sowohl die Unfähigkeit, einen informed consent zu geben als auch die erhöhte Wahrscheinlichkeit, unter Zwang oder durch Manipulation zuzustimmen, also eine unfreiwillige Zustimmung, sind personenbezogene Herangehensweisen an das Konzept der Vulnerabilität. Sie sollen in dieser Arbeit neben Hursts und Kottows Herangehensweisen als weitere Kriterien für Vulnerabilität miteinbezogen werden.

Es gibt AutorInnen, die erläutern, dass das Konzept des informed consent jedoch für eine ethisch vertretbare klinische Forschung nicht ausreicht. Emanuel et al. kritisieren: „While informed consent is necessary in most but not all cases, in no case is it sufficient for ethical clinical research“ (Emanuel et al. 2000, S. 2701). Einige stark umstrittene ethische Konflikte, wie beispielsweise klinische Forschung in Entwicklungsländern, die Verwendung von Placebos, Phase I-Studien, der Schutz von Gemeinschaften und das Einbeziehen von Kindern in Forschung können nicht gänzlich mit informed consent gelöst 
werden (Emanuel et al. 2000). Deswegen schlagen Emanuel et al. neben dem informed consent weitere sechs Voraussetzungen für ethische Forschung vor. Diese Voraussetzungen lauten: gesellschaftlicher oder wissenschaftlicher Nutzen, wissenschaftliche Validität, faire Rekrutierung von ProbandInnen, günstige Nutzen-Risiko-Verhältnisse, unabhängige Überprüfung und Bewertung, informed consent und Respekt für potenzielle und rekrutierte ProbandInnen (Emanuel et al. 2000). Für diese Arbeit ist der Aspekt des angemessenen Nutzen-Risiko-Verhältnisses von besonderer Bedeutung. Als zugrundeliegendes ethisches Prinzip geben Emanuel et al. Nicht-Ausbeutung an, was insbesondere im Kontext transnationaler Forschung wichtig ist (siehe hierzu Kapitel 3.3). Da weitere Voraussetzungen unabhängig von informed consent notwendig sind, um ethische Forschung gewährleisten zu können (Emanuel et al. 2000), bedeutet dies, dass informed consent nicht ausreicht, um ProbandInnen hinreichend zu schützen. Folglich sind weitere Schutzmaßnahmen notwendig. Im Umkehrschluss bedeutet dies jedoch auch, dass ein Fehlen von informed consent nicht der einzige Grund für unzureichenden Schutz darstellt. Folglich könnten Personen, die zum Beispiel anfällig dafür sind, bei Forschung mit unangemessenem Nutzen-Risiko-Verhältnis teilzunehmen, besonderen Schutz benötigen, obwohl der Prozess des informed consent gültig abgelaufen ist. Diese Personen müssten daher trotz des gültigen informed consent als vulnerabel gelten, da sie besonderen Schutz benötigen und dies die Grundlage für Vulnerabilität in dieser Arbeit darstellt. Somit kann Vulnerabilität nicht ausreichend mit der Unfähigkeit, informed consent zu geben oder mit der Ungültigkeit des informed consent, zum Beispiel durch Zwang, geklärt werden. Daher müssen die Definitionen von Vulnerabilität, die informed consent in den Fokus rücken, ergänzt werden. Da aufgezeigt wurde, dass weder die Prädisposition, zusätzliches Leid zu erfahren, noch informed consent als personenbezogene Kriterien für Vulnerabilität im Kontext von Forschung mit Frauen in Entwicklungsländern ausreichen, muss ein weiterer personenbezogener Ansatz folgen.

Hilfreich ist hier die Betrachtungsweise von Macklin. Sie bezieht sich hauptsächlich auf Vulnerabilität im Kontext internationaler Biomedizin und auf Vulnerabilität von Frauen bedingt durch kulturelle Gegebenheiten (Macklin 2003). Deswegen ist ihre Sichtweise gerade für die Beantwortung der Fragestellung dieser Arbeit besonders wichtig. Macklin gibt keine eigene Definition von Vulnerabilität. Sie bezieht sich auf die Beschreibungen der Deklaration von Helsinki (2008) und der International Ethical Guidelines for Biomedical Research der CIOMS, die in Kapitel 4.1 genauer erläutert werden und Vulnerabilität mit informed consent verknüpfen. An dieser Stelle ist jedoch der von Macklin dargestellte Zusammenhang von Vulnerabilität und Ausbeutung von großer Bedeutung, der von ihr folgendermaßen eingeleitet wird: „What makes individuals, groups, or even entire countries vulnerable? And why is vulnerability a concern in bioethics? A simple answer to both questions is that vulnerable individuals and groups are subject to exploitation, and exploitation is morally wrong" (Macklin 2003, S. 472). Zu dieser Aussage ergänzt sie jedoch zwei Kritikpunkte. Zum einen hersche starke 
Unstimmigkeit, wie Ausbeutung zu definieren sei. Laut Macklin entstehe Ausbeutung dann, wenn reiche oder mächtige Individuen oder Agenturen die Armut, Machtlosigkeit oder Abhängigkeit anderer ausnutzen, indem diese den Zielen der Reichen oder Mächtigen dienen ohne adäquate nutzbringende Kompensation zu erhalten (Macklin 2003). Eine detailliertere Erläuterung über Ausbeutung im Kontext transnationaler Biomedizin und der Zusammenhang zu Vulnerabilität werden in Kapitel 3.3 folgen. Zum anderen müsse laut Macklin beachtet werden, dass nicht alle unrechten Handlungen ausbeuterisch sind. Ein Beispiel hierfür ist eine Placebo-kontrollierte Studie, die den lokal üblichen statt den international bestmöglichen Standard als Placebo einsetzt. Hierbei könnten ProbandInnen einen vermeidbaren Schaden erleiden, es muss sich jedoch nicht um eine ausbeutende Situation gehandelt haben. Demnach zeigt sich die Notwendigkeit, die zuvor aufgeführten Aspekte Prädisposition für zusätzliches Leid und informed consent als Kriterien für Vulnerabilität im Kontext transnationaler Biomedizin beizubehalten.

Macklin beschreibt, wie Vulnerabilität im Rahmen internationaler Forschung entstehen kann:

„[...] research subjects in the host country might be vulnerable by virtue of their low educational level or lack of familiarity with modern scientific concepts, their poverty or powerlessness, and therefore open to exploitation in some manner" (Macklin 2003, S. 474).

Laut Macklin kann Vulnerabilität folglich zu Ausbeutung führen. In dieser Arbeit sollen Individuen und Personengruppen, die anfällig dafür sind, ausgebeutet zu werden, als vulnerabel gelten. Die Anfälligkeit, ausgebeutet zu werden, dient daher als ein weiterer personenbezogener Ansatz von Vulnerabilität.

\section{Kritik am Konzept der Vulnerabilität}

An dieser Stelle ist es wichtig zu erwähnen, dass nicht alle BioethikerInnen das Konzept der Vulnerabilität für sinnvoll halten. Levine zum Beispiel kritisiert die Entwicklung in der Bioethik, dass zu viele Personengruppen als vulnerabel gelten würden. Hierdurch müsse inzwischen fast jeder als vulnerabel gelten und das Konzept verliere somit an Aussagekraft und Bedeutung (Levine et al. 2004, S. 46). Hier nennt sie zum Beispiel Gruppen wie Arbeitslose, Personen, die Sozialhilfe empfangen, Obdachlose, Nomaden oder PatientInnen mit unheilbaren Krankheiten, die als vulnerabel bezeichnet würden (Levine et al. 2004, S. 46). Sie ist der Meinung, dass ein solches Konzept von Vulnerabilität zu weit gefasst sei (Levine et al. 2004, S. 46). Auf der anderen Seite kritisiert sie, dass das Konzept der Vulnerabilität gleichzeitig zu eng gefasst sei. Der Fokus würde fast ausschließlich auf den Eigenschaften einer Personengruppe liegen, die die Fähigkeit, informed consent zu geben, angeblich minimieren oder ganz aufheben würden (Levine et al. 2004, S. 46f). Dies könnte die Aufmerksamkeit von den Gegebenheiten der Studie und dem sozialen oder ökonomischen Kontext ablenken, wodurch die ProbandInnen Schaden erleiden könnten (Levine et al. 2004, S. 46). Als dritten Punkt kritisiert sie, dass das Konzept der Vulnerabilität ganze Personengruppen stereotypisiert. Hierbei gäbe es keine 
Unterscheidung zwischen Individuen innerhalb der Gruppe, die tatsächlich spezielle Eigenschaften haben, die besondere Beachtung finden müssten und Individuen, die nicht sonderlich geschützt werden müssten (Levine et al. 2004, S. 47). Als Beispiele gibt Levine an, dass es fragwürdig sei alle Schwangeren, alle Menschen, die in Armut leben und alle Mitglieder ethnischer Minderheiten zwangsweise als vulnerabel zu bezeichnen (Levine et al. 2004, S. 47). Macklin vertritt die Annahme, dass ganze Länder vulnerabel sein könnten (Macklin 2003). Dies stellt allerdings eine zu weite Auffassung von Vulnerabilität dar. Resnik bringt ein gutes Beispiel, um diesen Konflikt zu verdeutlichen. Würden alle ProbandInnen in Entwicklungsländern als vulnerabel bezeichnet, gäbe es keinen Unterschied zwischen einem weißen Geschäftsmann in Südafrika und einer schwarzen Frau, die in einem kleinen Dorf in Äthiopien lebt (Resnik 2004). Außerdem könnten manche Personen laut Levine nur in gewissen Situationen vulnerabel sein, beispielsweise aufgrund des Zeitpunkts der Studienteilnahme. Dies gilt zum Beispiel für Schwangere in den Wehen. Oder aufgrund emotionaler Beeinflussung im Rahmen einer Studie, die eine Krankheit untersucht, an der vor kurzem eine wichtige Bezugsperson eines/r ProbandIn gestorben ist (Levine et al. 2004, S. 47). Denn nicht alle, die zu einer vulnerablen Gruppe zählen, wie zum Beispiel Schwangere, seien automatisch auch vulnerabel. Zudem müsse die zentrale Frage lauten, welche Art von besonderem Schutz notwendig ist. Stattdessen, kritisiert sie, würde es bisher hauptsächlich darum gehen, welche Gruppe noch zur Kategorie vulnerabel hinzugefügt wird. Daher müsste man in der Bioethik breiter diskutieren, wie ein sinnvolles Konzept der Vulnerabilität aussehen könnte. Und darüber hinaus, wie gezieltere Schutzmaßnahmen dort bereitgestellt werden könnten, wo das Konzept der Vulnerabilität versagt (Levine et al. 2004, S. 48).

In dieser Arbeit soll Levines Kritik aufgegriffen werden und daher Vulnerabilität genauer definiert werden. Auf der einen Seite sollen die in Kapitel 3.4 vorgestellten Vulnerabilitätskriterien ausreichend sein, um zumindest vulnerable Personengruppen im Kontext transnationaler Biomedizin identifizieren zu können, um daraufhin konkrete Schutzmaßnahmen entwickeln zu können. Auf der anderen Seite sollen die zu erarbeitenden Vulnerabilitätskriterien nicht $\mathrm{zu}$ weit gefasst sein, um einer möglichen Stereotypisierung entgegenzuwirken. Zudem ist es zunächst plausibel, dass die Konzipierung der Studie und die äußeren Umstände einen großen Einfluss darauf haben, ob eine Person als vulnerabel gelten sollte oder nicht. Deswegen sollen sowohl der Kontext der Studie als auch der Kontext der Person oder Personengruppe in die Vulnerabilitätskriterien mit einbezogen werden. Es ist jedoch unwahrscheinlich, dass eine genaue und individuelle Berücksichtigung aller Lebensumstände für die Beurteilung der Vulnerabilität potenzieller ProbandInnen praktisch umsetzbar wäre. Deswegen erscheint es wichtig, vulnerable Individuen in Personengruppen einzuteilen, falls sie durch eine bestimmte Eigenschaft, die sie teilen, vulnerabel sind. Hierfür sind die Eigenschaften einer Person für die Aufstellung der Vulnerabilitätskriterien von Bedeutung, wie zum Beispiel die Unfähigkeit, informed consent zu geben bei komatösen PatientInnen. Abschließend verfolgt auch diese Arbeit das Ziel, angemessene 
Schutzmaßnahmen mehr in den Vordergrund zu stellen. Aus dem Grund, warum jemand als vulnerabel gilt, sollte ein Lösungsansatz entwickelt werden. Deswegen folgt in Kapitel 6.2, nach der Anwendung der Vulnerabilitätskriterien anhand der Fallstudien, die genauere Betrachtung möglicher Schutzmaßnahmen mit Blick auf die jeweiligen Vulnerabilitätskriterien in Kapitel 3.4. Beispiele für mögliche gezielte Schutzmaßnahmen für vulnerable Personengruppen sind: Die Verbesserung des Prozesses des informed consent bei Personen, die Schwierigkeiten haben, studienrelevante Informationen zu verstehen und gegebenenfalls der Ausschluss vulnerabler Gruppen von Studien.

Nach der Betrachtung verschiedener Herangehensweisen an das Konzept der Vulnerabilität und einer kritischen Auseinandersetzung mit diesen, sollen nun spezifische Aspekte für Vulnerabilität im Rahmen medizinischer Studien mit Frauen erläutert werden.

\section{$\underline{3.2 \text { Vulnerabilität im Kontext medizinischer Forschung mit Frauen }}$}

Eine mögliche Vulnerabilität kompetenter Erwachsener wird häufig im Zusammenhang mit gebärfähigen oder schwangeren Frauen debattiert (Wild 2007; Kopelman 2004, S. 2366f). Viele Richtlinien in der Bioethik bezeichnen gebärfähige und vor allem schwangere Frauen als vulnerabel und fordern mehr regulierenden Schutz, selbst wenn dieser die Entscheidungsmöglichkeiten dieser Frauen einschränkt. Dadurch sollen Föten, Neugeborene, schwangere und stillende Frauen vor den Risiken, die eine Studienteilnahme mit sich bringen, geschützt werden. Es gibt jedoch keine Einigung darüber, welchen Stellenwert das Recht der Selbstbestimmung dieser Frauen einnimmt (Kopelman 2004, S. 2367). Ein kategorischer Ausschluss Schwangerer von der Forschung würde diesen Frauen nämlich das Recht absprechen, sich selbstbestimmt für oder gegen eine Studienteilnahme entscheiden zu können. Der Ausschluss vieler Frauen von medizinischer Forschung bringt weitere Nachteile mit sich. Cook erläutert, dass Frauen im Vergleich zu Männern biologische Unterschiede aufweisen, wie zum Beispiel Körperform, Größe der Organe und Verteilung des Fettgewebes. Hierdurch könnten zum Beispiel Medikamente verschiedene Wirkungen bei Frauen und Männern auslösen (Cook 1994). Zusätzlich weisen beispielsweise manche Erkrankungen bei Frauen eine erhöhte Prävalenz auf oder gehen mit einem schwerwiegenderen Verlauf einher (Cook 1994). Seit den 80er Jahren gibt es das Forschungsfeld der sogenannten Gender Medicine, das das Ziel verfolgt sex, gender ${ }^{2}$ und Gesundheit vermehrt in den Fokus biomedizinischer Forschung zu rücken. Hierbei sollen geschlechtsspezifische Gesundheitsbedürfnisse von Frauen und Männern in klinischer Forschung und Praxis beachtet werden (Klinge

\footnotetext{
${ }^{2}$ Man unterscheidet das biologische, anatomische Geschlecht (sex) und die damit verbundenen gesellschaftlichen Erwartungen und Zuschreibungen, nämlich das soziale Geschlecht (gender). (Vgl. zum Beispiel Degele 2008, S. 67)
} 
2010). Ein weiterer Grund Frauen nicht von medizinsicher Forschung auszuschließen ist, dass eine Forschungseilnahme der einzige Zugang zu subventionierten Medikamenten und Therapiemöglichkeiten sein könnte. Hiervon könnten diese Frauen also im Gegensatz zu Personen, die nicht als vulnerabel gelten, nicht profitieren.

Da es hier insbesondere um die Vulnerabilität von Frauen in Indien im Kontext transnationaler Biomedizin geht, würde es den Rahmen der Arbeit sprengen, eine mögliche Vulnerabilität schwangerer Frauen generell genau zu erörtern. Dies ist weit komplexer, da mögliche Risiken einer Studie nicht nur die Frauen, sondern auch den Fötus und die Person, die der Fötus eines Tages werden könnte, betreffen. An dieser Stelle ist es vielmehr von Bedeutung, eine mögliche Vulnerabilität von Frauen unabhängig von Schwangerschaft oder Gebärfähigkeit zu erläutern. Dies baut auf der Vermutung auf, dass auch soziale Gegebenheiten dazu führen könnten, dass Frauen in bestimmten Kontexten als vulnerabel gelten können. Jenseits von Schwangerschaft und Gebärfähigkeit müssen also personenbezogene und den Studienkontext betreffende Kriterien für Vulnerabilität gesucht werden, die jedoch auch durch bestimmte Aspekte des Frauseins bedingt sein können. Inwiefern sozioökonomische Gegebenheiten und weitere Faktoren dazu führen könnten, dass Frauen als vulnerabel gelten sollten, wird daher im folgenden Abschnitt erläutert.

Bunch beschreibt beispielsweise, dass die Menschenrechte von Frauen auf unterschiedliche Arten missachtet werden und meist sei dies im Frausein begründet (Bunch 1990). Frauen werden in vielen Bereichen benachteiligt, diskriminiert und gar lebensbedrohlichen Situationen ausgesetzt. Hierzu einige Beispiele: Gewalt gegenüber Frauen ist ein weltweites Problem. Je nach Region erreicht der Prozentsatz der Frauen bezüglich aller Frauen in der jeweiligen Region, die mindestens einmal in ihrem Leben Opfer physischer Gewalt werden, teilweise bis zu 59\%. (United Nations Statistics Division 2010c). Diese Frauen werden aufgrund ihres Geschlechts als Opfer ausgewählt (Bunch 1990). Ein weiteres eindrückliches Beispiel ist der gezielte Schwangerschaftsabbruch weiblicher Föten aufgrund des Geschlechts. Vor allem in Indien stieg die Zahl der Abtreibungen aufgrund des Geschlechts in den letzten Jahren dramatisch an (Dennerstein 2000). Bedingt durch kulturelle, sozioökonomische und religiöse Faktoren bevorzugen viele Familien in Indien die Geburt eines Sohnes (Dennerstein 2000). Sozioökonomische Nachteile und patriarchalische Gesellschaftsstrukturen sind unter anderem Gründe für die Verletzung der Menschenrechte von Frauen (Bunch 1990). Dies sollte gerade für den Kontext dieser Arbeit bei der Aufstellung der Kriterien für Vulnerabilität mit in Betracht gezogen werden.

Betrachtet man die Gesundheit von Frauen weltweit und Faktoren, die sie beeinflussen, zeigt sich auch in diesem Bereich eine Diskriminierung von Frauen basierend auf ihrem sozialen Geschlecht (Cook 1994). Die Auswirkungen vielfältiger Benachteiligungen und Abwertungen von Frauen innerhalb rechtlicher, religiöser und kultureller Traditionen sowie sozioökonomische Systeme führen dazu, dass vielen Frauen Gesundheit vorenthalten wird (Cook 1994). Ein Beispiel hierfür ist die hohe 
Müttersterblichkeit, die 2005 über eine halbe Million betrug, hiervon die Mehrzahl der Fälle in Entwicklungsländern (United Nations Statistics Division 2010b). Direkte Gründe sind das Fehlen der medizinischen Versorgung für Schwangere und Gebärende. Weitere zugrundeliegende Ursachen sind körperliche Erschöpfung und Blutarmut bedingt durch viele Geburten und kurze Zeitintervalle zwischen diesen. Außerdem sind strukturelle Gegebenheiten, wie zum Beispiel Armut, ursächlich für die hohe Müttersterblichkeit (Cook 1994). Hierzu einige Beispiele aus den Statistiken der UN von 2010 (United Nations Statistics Division 2010a): Alleinerziehende Mütter leben häufiger in Armut als alleinerziehende Väter. In westlichen Regionen sind ältere Menschen, die in Armut leben, häufiger Frauen. In vielen Ländern Afrikas und Asiens gibt es für Frauen rechtliche Beschränkungen Eigentum zu besitzen. In vielen Entwicklungsländern haben Frauen seltener finanzielles Einkommen als Männer und viele verheiratete Frauen haben kein Mitspracherecht über die Ausgaben ihres Einkommens. Da Armut häufig mit einem schlechten Gesundheitszustand einhergeht und Frauen überproportional häufiger in Armut leben, zeigt sich ein Zusammenhang zwischen der Gesundheit von Frauen und deren Lebensumständen (Jacobson 1993). Menschen, die in Armut leben, haben weniger finanzielle Mittel zur Verfügung um Nahrung, sauberes Wasser, geeignete Kleidung und eine sichere Unterkunft zu erwerben. Des Weiteren haben sie weniger Zugang zu Bildung und politischer Macht, was jedoch wichtig ist, um den eigenen Gesundheitszustand zu verbessern und zu schützen (Jacobson 1993).

Zieht man die oben genannten Beispiele für die Nichtbeachtung der Menschenrechte von Frauen und für die Nachteile von Frauen im Gesundheitsbereich in Betracht, stellt sich die Frage nach der ethischen Bedeutung im Kontext medizinischer Forschung mit Frauen. Auf der einen Seite ist medizinische Forschung mit Frauen notwendig, um den Gesundheitsbedürfnissen von Frauen besser gerecht zu werden. Die Nichtbeachtung dieser Notwendigkeit könnte eine studienkontextbezogene Ursache für Vulnerabilität sein. Auf der anderen Seite könnten Frauen jedoch durch sozioökonomische Nachteile und patriarchale Gesellschaftsstrukturen im Kontext medizinischer Forschung eine vulnerable Gruppe darstellen. Würde man Frauen aufgrund der oben genannten Nachteile und Diskriminierungen als vulnerabel bezeichnen, würde dies nicht automatisch bedeuten, dass man für den Ausschluss von Frauen in medizinischen Studien plädieren müsste. Dies wäre nämlich nur eine der möglichen Schutzmaßnahmen für vulnerable Gruppen. Weitere mögliche Schutzmaßnahmen werden in Kapitel 6.2.2 erläutert.

Personenbezogene Faktoren, die zu einer möglichen Vulnerabilität von Frauen führen könnten, lassen sich in soziale oder körperliche Aspekte einteilen: Soziale Aspekte beinhalten beispielsweise, dass Frauen häufiger in Armut leben und dadurch im Kontext medizinischer Forschung ausgebeutet werden könnten. Außerdem könnte es sein, dass Frauen, die keinen Zugang zu Bildung haben, die Risiken und Nutzen einer Studienteilnahme nicht vollständig verstehen. Hier wäre folglich ein unzureichender informed consent die Ursache für eine mögliche Vulnerabilität. 
Körperliche Aspekte umfassen hingegen Faktoren, die eine erhöhte körperliche Verletzbarkeit, im Sinne einer potenziellen körperlichen Verletzung, bedingen. Hierbei meine ich keine (vermeintliche) körperliche Schwäche aufgrund des Geschlechts, sondern eine erhöhte Verletzbarkeit durch beispielsweise eine Schwangerschaft. Außerdem könnten soziale Gegebenheiten dazu führen, dass sich körperliche Verletzbarkeit manifestiert. In manchen Regionen der Welt ist es zum Beispiel üblich, dass Mädchen und Frauen weniger Nahrung erhalten als Jungen und Männer. Die Mangelernährung schwächt den Körper (Merchant und Kurz 1993). Zusätzlich wird der Körper durch häufige Geburten und zu kurze Intervalle zwischen diesen geschwächt. Eine erhöhte körperliche Verletzlichkeit könnte also dazu führen, dass diese Frauen während einer Studienteilnahme ein erhöhtes Risiko aufweisen, körperlichen Schaden zu erleiden. Um dies zu verhindern, benötigen sie besondere Schutzmaßnahmen im Rahmen der Studienteilnahme und könnten demnach als vulnerabel bezeichnet werden.

Abschließend gilt es jedoch klarzustellen, dass Frauen nicht per se als vulnerabel gelten sollten, da Geschlecht kein Kriterium für Vulnerabilität darstellt (Macklin 2003). Aspekte, die Frauen vulnerabel machen könnten, treffen nicht auf alle Frauen der Welt zu, sondern sind Kriterien, wie bisher deutlich geworden sein sollte, die spezifische Merkmale von bestimmten Frauen berücksichtigen, eben abhängig von Studienkontext und Person.

Um angemessene Kriterien für Vulnerabilität hinsichtlich Forschung mit Frauen in Entwicklungsländern aufstellen zu können, müssen nach der Beleuchtung spezifischer Aspekte bezüglich Forschung mit Frauen nun zusätzlich Faktoren untersucht werden, die ethische Konflikte im Kontext medizinischer Forschung mit Menschen in Entwicklungsländern betreffen und möglicherweise zu Vulnerabilität der ProbandInnen führen könnten.

\section{$\underline{3.3 \text { Vulnerabilität im Kontext medizinischer Forschung in Entwicklungsländern und der }}$ Begriff der Ausbeutung}

Klinische Studien, die mit ProbandInnen in Entwicklungsländern durchgeführt werden, werfen zahlreiche ethische Konflikte auf. Einige dieser Konflikte, die eine mögliche Vulnerabilität der StudienteilnehmerInnen bedingen oder beeinflussen könnten, sollen im Folgenden erörtert werden:

\section{1) Ausbeutung}

Zunächst gilt es, die mögliche Ausbeutung von StudienteilnehmerInnen, die aus ethischer Sicht eines der Hauptprobleme im Kontext transnationaler Forschung darstellt, genauer zu untersuchen und im Anschluss an den bereits in Kapitel 3.1 vorgestellten Zusammenhang von Ausbeutung und Vulnerabilität zu erläutern (Macklin 2003; Ballantyne 2010). Zu Beginn muss jedoch genauer geklärt 
werden, was Ausbeutung bedeutet. Exploitation aus dem Jahre 1996 von Alan Wertheimer gilt als Standardwerk zu Ausbeutung und viele AutorInnen beziehen sich im Kontext medizinischer Forschung in Entwicklungsländern darauf. Als Gemeinsamkeit verschiedener Betrachtungsweisen von Ausbeutung fasst Wertheimer zusammen, dass Ausbeutung eine Interaktion zwischen A und B sei, wobei B von A unfair ausgenutzt wird. Er schreibt: „Let us start with the claim that A exploits B when A takes unfair advantage of B“ (Wertheimer 1996, S. 16). Ein unfaires Ausnutzen zeige sich entweder durch das Resultat oder den eigentlichen Ablauf einer Interaktion. Das Ergebnis der Interaktion könne entweder unfair sein, weil A von der Interaktion profitiert und B Schaden nimmt oder As Nutzen vergleichsweise übermäßig erscheint. Der Ablauf könne zu einem unfairen Ausnutzen von B führen, wenn dieser zum Beispiel unter Zwang steht oder von A manipuliert wurde (Wertheimer 1996, S. 16). Wertheimer unterscheidet zwischen Ausbeutung, die B ausschließlich schadet, und Ausbeutung, die beiden Parteien einen Nutzen bringt. Hierbei ist der Effekt der Interaktion für B entscheidend. Eine Interaktion, von der beide profitieren, ist dann ausbeutend, wenn das Ergebnis der Interaktion für B unfair ist (Wertheimer 1996, S. 20). Dies bedeutet, B profitiert durch die Interaktion verglichen zu einem Nichtstattfinden der Interaktion, aber das Fairness Level betrachtend bedeutet die Interaktion trotzdem einen Verlust für B (Wertheimer 1996, S. 22f). Ausbeutung findet laut Wertheimer auf einem Mikrolevel statt, also zwischen zwei individuellen Parteien. Daher könne es selbst in einer gerechten Gesellschaft, die laut Wertheimer ein Makrolevel darstellt, Ausbeutung geben, nämlich auf dem Mikrolevel (Wertheimer 1996, S. 9).

Ob medizinische Studien in Entwicklungsländern, gesponsert von Pharmafirmen aus Industriestaaten, ausbeutend sind, hängt somit von der jeweiligen Studie ab. Die Tatsache, dass Firmen aus Industriestaaten, Studien in Entwicklungsländern durchführen, deren Gesetzgebung und Gesellschaft Ungerechtigkeiten möglicherweise eher zulassen, reicht per se nicht aus, um von Ausbeutung sprechen zu können. Im Rahmen von Studien in Entwicklungsländern kann davon ausgegangen werden, dass es sich in den meisten Fällen um gegenseitig nutzenbringende Transaktionen handelt, da sowohl Pharmafirmen als auch ProbandInnen, beispielsweise in Form von kostenfreier medizinischer Versorgung, von der Transaktion profitieren (Ballantyne 2010). Es handelt sich jedoch um eine ausbeuterische Interaktion, wenn die ProbandInnen für ihre Studienteilnahme keinen fairen Nutzen erhalten (Gbadegesin und Wendler 2006). Ein fairer Nutzen für ProbandInnen hängt sowohl von dem Nutzen für die Pharmafirma im Vergleich zum Nutzen der ProbandInnen, als auch von den Nachteilen einer Studienteilnahme ab (Gbadegesin und Wendler 2006). Hierbei gilt, dass bei einer Studie, die mit vielen oder schwerwiegenden Nachteilen einhergeht, auch ein sehr hoher Nutzen bereitgestellt werden sollte. Es ist wichtig klarzustellen, dass verschiedene Arten von Nutzen möglich sind. Beispiele für mögliche Formen von Nutzen für eine Studienteilnahme sind: Das Bereitstellen eines als erfolgreich getesteten Medikaments, der Ausbau von Infrastruktur hinsichtlich der Gesundheitsversorgung, 
kostenfreier Zugang zu medizinischer Versorgung oder eine angemessene Aufwandsentschädigung. Ausbeutung im Kontext transnationaler Biomedizin könnte durch das Bereitstellen eines fairen Nutzens verhindert werden (Gbadegesin und Wendler 2006).

An dieser Stelle drängt sich selbstverständlich die Frage auf, wer die Fairness des Nutzens für ProbandInnen beurteilt und anhand welcher Kriterien. Hierzu gibt es verschiedene Lösungsansätze:

\section{Free market}

Ein erster möglicher Lösungsansatz wäre, dass die Fairness des Nutzens durch StudienteilnehmerInnen selbst bestimmt werden müsste. Demnach würden potenzielle ProbandInnen frei entscheiden, ob sie an einer Studie teilnehmen und ob der Nutzen fair im Vergleich zu den gegebenen Risiken sei (Participants in the Conference on Ethical Aspects of Research in Developing Countries 2004). Diesen Lösungsansatz bezeichnet Ballantyne als free market, da hierbei die an einer Transaktion beteiligten Parteien den Nutzen im Vergleich zu anderen verfügbaren Transaktionen frei festlegen können. Zunächst ist es einleuchtend, dass potenzielle StudienteilnehmerInnen bei der Festlegung eines fairen Nutzens beteiligt werden müssen. Allerdings kann durch beidseitige Zustimmung weder die Fairness des Nutzens garantiert werden, noch Ausbeutung verhindert werden (Ballantyne 2010). Befinden sich potenzielle StudienteilnehmerInnen in einer verzweifelten Situation, wie dies besonders im Kontext internationaler Forschung der Fall sein könnte, stimmen sie möglicherweise aus rationalen Gründen einer Studienteilnahme zu, unabhängig davon, ob sie den Nutzen als fair einschätzen (Ballantyne 2010). Zudem könnte dieser Lösungsansatz dazu führen, dass potenzielle Forschungspopulationen, die um extern gesponserte Studien konkurrieren, ihre Ansprüche an einen fairen Nutzen jeweils unterbieten (Ballantyne 2010).

\section{Hypothetical market}

Ein zweiter möglicher Lösungsansatz zu Fairness im Kontext transnationaler Biomedizin wäre der des bypothetical market. Hierbei wird ein fairer Nutzen anhand eines hypothetischen, idealen und wettbewerbsfähigen Marktes ermittelt. Eine ausbeutende Transaktion sähe hier wie folgt aus:

„In exploitative transactions, the powerful party uses an inequality of bargaining power to achieve more than they would have fairly achieved in a competitive market and the vulnerable party receives comparatively less than they would have achieved in a competitive market" (Ballantyne 2010, S. 28).

Demnach ist der Nutzen dann fair, wenn die beteiligten Parteien keinen größeren beziehungsweise geringeren Nutzen im Vergleich zu einer Transaktion mit einer anderen Partei erhalten (Wertheimer 1996, S. 232). Als Beispiel hierzu nennt Ballantyne den Verkauf eines Hauses unter Verwandten. Liege der Preis deutlich unter dem Preis, den ein anonymer Käufer für das Haus gezahlt hätte, könnte die 
verkaufende Partei hierbei ausgebeutet worden sein. Hinsichtlich der Verhinderung von Ausbeutung im Rahmen transnationaler Biomedizin würde dies bedeuten, dass Pharmafirmen keinen größeren Nutzen aus Forschung mit ProbandInnen in Entwicklungsländern im Vergleich zu Forschung in Industriestaaten ziehen dürften. Ballantyne kritisiert jedoch, dass es für das Abschätzen eines fairen Nutzens im Rahmen von transnationaler Forschung keine klaren Referenzpunkte gäbe, wie dies zum Beispiel bei dem Verkauf von Häusern der Fall wäre (Ballantyne 2010). Zum einen könne Forschung in Industriestaaten nicht sinnvoll mit Forschung in Entwicklungsländern verglichen werden, da ProbandInnen in Industriestaaten nicht an Studien teilnehmen, um Zugang zu grundlegender medizinischer Versorgung zu erhalten, die sonst nicht verfügbar wäre. Zum anderen gibt es keine empirischen Daten, die den hypothetischen Wert von möglichen Formen von Nutzen im Rahmen internationaler Forschung abschätzen könnten und somit als Referenzpunkte für fairen Nutzen dienen könnten (Ballantyne 2010).

\section{Maximin theory}

Ein dritter Lösungsansatz, um Fairness im Kontext internationaler Forschung zu erreichen, wäre die sogenannte Maximin theory. Diese lautet wie folgt: „Maximin requires that the benefits of cooperative activity be distributed in a manner that is to the greatest advantage of the worst-off party to the transaction“ (Ballantyne 2010, S. 29). Dieses Prinzip sei besonders geeignet für eine faire Verteilung im Falle von besonderer Vulnerabilität und enormer Machtasymmetrie der beteiligten Parteien. Ballantyne erläutert, dass dann eine moralische Asymmetrie vorherrsche, wenn eine Partei aufgrund sozialer Umstände gezwungen wäre für sogenannte basic goods eine Transaktion einzugehen, wobei die andere Partei die Transaktion primär für wirtschaftlichen Gewinn eingeht. Hier lägen dann ungleiche Ausgangspositionen der jeweiligen Parteien vor. Als Beispiele für sogenannte basic goods gibt sie Gesundheit, Bildung und Sicherheit an (Ballantyne 2010). Betrachtet man die Situation transnationaler Biomedizin, ist es plausibel, dass es sich in den meisten Fällen um eine Transaktion handelt bei der beide Parteien mit sehr unterschiedlichen Ausgangsituationen starten und demnach die Maximin theory greifen könnte. Pharmafirmen versprechen sich einen wirtschaftlichen Erfolg aus der Forschung, wobei ProbandInnen in Entwicklungsländern möglicherweise nur teilnehmen, um grundlegende medizinische Versorgung, also basic goods, zu erhalten. Folglich müssten laut der Maximin theory ProbandInnen in Entwicklungsländern bei der Verteilung des Nutzens, der aus der Forschung resultiert, im Vergleich zu den Pharmafirmen bevorzugt werden. Als praktische Umsetzung für die Maximin theory hinsichtlich transnationaler Biomedizin schlägt Ballantyne vor, eine Steuer für transnationale Forschung einzurichten. Hierbei müssten Pharmafirmen, die Forschung in Entwicklungsländer auslagern, einen gewissen Prozentsatz der Studienkosten, gemessen an einer Studiendurchführung in dem jeweiligen Entwicklungsland, an die Forschungspopulation zahlen (Ballantyne 2010). Als Beispiel errechnet 
Ballantyne die zu zahlende Steuer für eine Studie in Uganda und wendet verschiedene Prozentsätze an (10\% bis zu 90\%). Sie zeigt hierbei, dass eine Pharmafirma bei einer Studiendurchführung in Uganda im Vergleich zu einer Studiendurchführung in den USA, trotz einer Steuer in Höhe von 90\% der Studienkosten (in Uganda), circa ein Drittel der Kosten einsparen würde. Die Steuer könnte im Gastland beispielsweise für den Aufbau medizinischer Infrastruktur genutzt werden (Ballantyne 2010). Jedoch auch dieser Lösungsansatz wirft viele Fragen und Kritikpunkte auf. Wertheimer et al. fordern, dass ein Lösungsansatz zur Verhinderung von Ausbeutung für alle ausbeutenden Transaktionen anwendbar sein solle (Wertheimer et al. 2010). Ballantynes Ansatz bezieht sich jedoch nur auf Transaktionen, die Parteien mit großer Machtasymmetrie betreffen. Des Weiteren fragen Wertheimer et al., ob die Forschungssteuer nicht in erste Linie an die Personen gehen sollte, die sich in der misslichsten Lage befinden, anstatt primär an Forschungsteilnehmer (Wertheimer et al. 2010). Zusätzlich ist die praktische Umsetzung der Forschungssteuer nicht eindeutig. Macklin wirft beispielsweise die Frage auf, wer die Höhe der Steuer festlegt und verteilt und wie dies wiederum überwacht wird (Macklin 2010).

Die Frage nach der idealen Lösung, um einen fairen Nutzen für ProbandInnen in Entwicklungsländer zu garantieren und hierdurch Ausbeutung im Rahmen transnationaler Biomedizin zu verhindern, kann im Rahmen dieser Arbeit nicht gänzlich beantwortet werden. Es könnte jedoch eine Kombination verschiedener Lösungsansätze sinnvoll sein (Macklin 2010).

Hinsichtlich Vulnerabilität und Ausbeutung muss an dieser Stelle noch erwähnt werden, dass Forschung mit einer vulnerablen Gruppe nicht zwingend darauf schließen lässt, dass diese Gruppe im Rahmen der Studienteilnahme ausgebeutet wird. Es ist nämlich möglich, dass ProbandInnen, die sich in einer misslichen Lage befinden und folglich vulnerabel sein könnten, trotzdem einen fairen Nutzen für ihre Studienteilnahme erhalten. Folglich würden diese ProbandInnen trotz ihrer Vulnerabilität nicht ausgebeutet (Gbadegesin und Wendler 2006). Es ist jedoch anzunehmen, dass Personen, die sich in einer misslichen Lage befinden, mit erhöhter Wahrscheinlichkeit einer Studienteilnahme mit unfairem Nutzen für sie zustimmen könnten. Demnach würden sie dann ausgebeutet. Folglich könnte die Anfälligkeit aufgrund bestimmter Lebensumstände einer Studienteilnahme mit unfairem Nutzen zuzustimmen, im Sinne einer Anfälligkeit ausgebeutet zu werden, als ein Kriterium für Vulnerabilität dienen.

\section{2) Behandlungsstandard bei Placebo-kontrollierten Studien}

Die Konzipierung Placebo-kontrollierter Studien stellt einen weiteren ethischen Konflikt transnationaler Biomedizin dar. Hierbei könnte die international bestmögliche oder die lokal übliche Behandlung als Standard für die Kontrollgruppe dienen (Emanuel et al. 2005). Dies bedeutet, dass 
ProbandInnen, die der Kontrollgruppe einer Studie zugeteilt werden, möglicherweise gar nicht behandelt werden, weil lokal keine Behandlung existiert, also Nicht-Behandlung als Standard gilt. Allerdings existiert international eine etablierte Therapie als Vergleich. Ein vielfach diskutiertes Beispiel sind HIV-Studien, die in Entwicklungsländern durchgeführt wurden (siehe hierzu Kapitel 1). Hierbei war das Ziel der Studien, ein geeignetes und bezahlbares Medikament zur Verhinderung der Übertragung von HIV von schwangeren Frauen auf deren Föten, zu finden. Probandinnen der Kontrollgruppe erhielten bei manchen dieser Studien ein Placebo, obwohl bereits ein Medikament existierte, das die Übertragung nachweislich senkt und in westlichen Ländern auch eingesetzt wird (Lurie und Wolfe 1997). Dies wird auch als ethisches Problem sogenannter doppelter Standards in der Forschungsethik beschrieben (Kottow 2003). Hiermit ist gemeint, dass die ethischen Standards für Studien in westlichen Ländern höher angesetzt und strenger bewertet werden im Vergleich zu den Standards bei Forschung mit Menschen in Entwicklungsländern.

\section{3) Zusätzliche medizinische Versorgung}

Ein weiterer ethischer Konflikt entsteht durch die Frage, ob man während einer Studie den ProbandInnen zusätzliche medizinische Versorgung bereitstellen sollte (Emanuel et al. 2005). Erkranken ProbandInnen beispielsweise während einer Studie zu HIV an Tuberkulose, stellt sich die Frage, wer für die Behandlung aufkommen sollte.

\section{4) Fremdnützige Forschung}

Studien, die in Entwicklungsländern durchgeführt werden, sollten auch für die dortigen Bedürfnisse relevant sein. Hinter dieser Forderung steht die Sorge, dass Medikamente, die vorrangig in Industriestaaten zur Behandlung von dort häufigen (z.B. Lifestyle-) Erkrankungen eingesetzt werden, mithilfe von ProbandInnen in Entwicklungsländern erforscht werden, obwohl die StudienteilnehmerInnen selbst nur kaum vom Ergebnis der Studie profitieren werden. Ein Beispiel hierfür könnte Forschung in Entwicklungsländern sein, um neue Therapieoptionen für Adipositas zu finden. Die Behandlung von Adipositas ist jedoch hauptsächlich für Bevölkerungen in Industriestaaten von Interesse und für die Gesundheitsprobleme in vielen Entwicklungsländern weniger relevant. Gleiches gilt für Medikamente, die so teuer sind, dass sie nur für PatientInnen in Industriestaaten zugängig sind. Hier stellt sich die Frage, ob es gerechtfertigt ist, dass die ProbandInnen nur durch eine Aufwandsentschädigung oder kurzzeitige medizinische Überwachung von der Studie profitieren, aber langfristig nur PatientInnen in Industriestaaten einen Nutzen daraus ziehen können. 


\section{5) Bereitstellung der Studienergebnisse}

Es gibt Unstimmigkeiten darüber, ob und in welcher Form ProbandInnen nach Abschluss einer Studie zu dem mit ihrer Hilfe entwickelten Medikament Zugang erhalten sollten. Die Bereitstellung eines neu entwickelten Medikaments für die StudienteilnehmerInnen könnte eine mögliche Form von fairem Nutzen sein. Folglich könnte mit dieser Form von Nutzen je nach Studienkontext Ausbeutung verhindert werden. Sehouty et al. beziehen sich auf Wertheimers Definition von Ausbeutung und erläutern, dass die Bereitstellung eines neuen Produktes jedoch nicht ausreicht, um den ProbandInnen einen fairen Nutzen per se zu garantieren. Somit könne Ausbeutung hierdurch nicht ausgeschlossen werden (Participants in the Conference on Ethical Aspects of Research in Developing Countries 2004). Dies erklärt sich wie folgt: Geht eine Studie beispielsweise mit einem sehr hohen Risiko einher, könnte nämlich zusätzlich zur Bereitstellung des neu entwickelten Medikaments eine weitere Form von Nutzen notwendig sein, um einen fairen Nutzen sicherzustellen. Außerdem könnte je nach Studienkontext und vor allem in Absprache mit potenziellen ProbandInnen auch eine andere Form von Nutzen, wie beispielsweise lebenslange Krankenversicherung oder eine angemessene Aufwandsentschadigung, sinnvoller sein, um einen fairen Nutzen zu garantieren. Zudem stellt sich in diesem Zusammenhang die Frage, ob der Zugang zu dem neu entwickelten Medikament für die ehemaligen StudienteilnehmerInnen aus ethischer Sicht zeitlich limitiert sein darf.

\section{6) Qualität des informed consent}

Abschließend ist die Qualität des informed consent $\mathrm{zu}$ hinterfragen, wenn medizinische Studien in Entwicklungsländern stattfinden. Hier stehen vorherrschende Armut, ein niedriger Bildungsstand, fehlender Zugang zu medizinischer Versorgung, kulturelle Unterschiede und damit zusammenhängende Zweifel bezüglich Kompetenz, Verstehen und Freiwilligkeit der ProbandInnen im Vordergrund sowie eine ungenügende Aufklärung von Seiten der ForscherInnen.

Potenzielle ProbandInnen, die sich in einer schwierigen finanziellen Lage befinden, könnten durch einen unangemessen hohen finanziellen Anreiz die Risiken einer Studie irrational gering einschätzen (Denny und Grady 2006). Emanuel et al. beschreiben, welche Bedingungen für einen unangemessenen Anreiz erfüllt sein müssen (Emanuel et al. 2005, S. 337). Zum einen müsse ein Anreiz angeboten werden, der wertvoll oder wünschenswert genug ist, um zum Beispiel in die Teilnahme einer Studie einzuwilligen. Das Angebot müsse außerdem übermäßig sein, sodass es unmöglich erscheint dieses abzulehnen. In einem weiteren Schritt führe der unangemessene Anreiz dazu, dass ein normales Urteilsvermögen eingeschränkt ist und dadurch eine Entscheidung getroffen wird, die ernsthaftes Leid hervorruft und entgegen der eigentlichen Interessen einer Person steht (Emanuel et al. 2005, S. 337). Das Anbieten von 1 Million Euro für die Teilnahme an einer Studie würde beispielsweise einen unangemessen Anreiz darstellen. Viele Menschen in Entwicklungsländern haben jedoch nicht mal 
Zugang zu medizinischer Versorgung und somit könnte die Teilnahme an einer Studie die einzige Behandlungsmöglichkeit darstellen. Selbst hier entsteht also der Konflikt, ob ein Anreiz, nämlich Zugang zu medizinischer Versorgung, unangemessen sein könnte. Emanuel et al. behaupten, dass die Sorge, ein unangemessen hoher Anreiz könnte bei transnationaler Forschung eine Rolle spielen, unbegründet sei (Emanuel et al. 2005, S. 338). Ein unangemessen hoher Anreiz sei nur dann gegeben, wenn die Risiken einer Studienteilnahme sehr hoch liegen, da nur so den ProbandInnen ernsthaftes Leid widerfahren könnte, was eine der Bedingungen für einen unangemessenen Anreiz darstellt. Also könne man durch ein Ausschließen von Studien, die ein zu hohes Risiko für die ProbandInnen bedeuten, auch ausschließen, dass StudienteilnehmerInnen in Entwicklungsländern durch einen unangemessen hohen Anreiz einer Studie zustimmen würden (Emanuel et al. 2005, S. 338). Hierbei muss jedoch beachtet werden, dass in Entwicklungsländern nicht ausgeschlossen werden kann, dass Studien mit einem erhöhten Risiko für die ProbandInnen durchgeführt werden. Zudem stellen sowohl die Einschätzung des Risikos als auch des Nutzens subjektive Komponenten dar und somit auch die Beurteilung, ob ein Anreiz unangemessen ist. Eine Person, die in einem Industrieland sozialisiert ist, kann außerdem schwer nachvollziehen, welche Prioritäten eine Person in einem Entwicklungsland bei der Entscheidungsfindung für oder wider eine Studienteilnahme setzt. Somit ist die Sorge, um einen ungültigen informed consent bedingt durch einen unangemessenen Anreiz, durchaus berechtigt.

Ein weiterer Grund für einen ethisch zweifelhaften informed consent im Kontext transnationaler Biomedizin könnte eine Zustimmung unter Zwang und somit eine unfreiwillige Zustimmung sein. Emanuel et al. verstehen unter Zwang, dass eine Person nur aufgrund einer Bedrohung einwilligt und hierdurch in eine schlechtere Position verglichen mit ihrer Ausgangssituation gebracht wird (Emanuel et al. 2005). Ein Beispiel hierfür wäre ein Raubüberfall, wobei eine Person nur einwilligt ihr Geld abzugeben, weil ihr Leben bedroht ist (Emanuel et al. 2005). Ein mögliches Beispiel für Zwang bei einer Studienteilnahme wäre eine Zustimmung aufgrund der Angst, bestimmte Vorteile medizinischer Versorgung zu verlieren oder für ein Verweigern der Studienteilnahme bestraft zu werden (Nelson und Merz 2002). Im Kontext transnationaler Forschung könnten ProbandInnen dann unter Zwang stehen, wenn sie möglicherweise durch den fehlenden Zugang zu medizinischer Versorgung nicht die Wahl haben, behandelt zu werden, ohne dies an eine Studienteilnahme zu knüpfen. Dies könnte zum Beispiel der Fall sein, wenn ProbandInnen nicht krankenversichert sind und sich medizinische Versorgung, die privat bezahlt werden müsste, nicht leisten können.

Manipulation von StudienteilnehmerInnen stellt ein weiteres Problem bezüglich der Qualität des informed consent dar. Hier stehen die Gültigkeit der Aufklärung sowie die Freiwilligkeit der Zustimmung zur Diskussion. Manipulation bedeutet, dass die Entscheidungsfindung einer Person durch Verändern der gegebenen Wahlmöglichkeiten oder durch Verändern der Wahrnehmung der selbigen beeinflusst wird (Nelson und Merz 2002). Hierbei können sich das Angebot einer Aufwandsentschädigung, freien 
Zugang zu medizinischer Versorgung oder das Darstellen mangelnder Alternativen auf die Entscheidung potenzieller ProbandInnen auswirken. Außerdem kann die Entscheidung für eine Studienteilnahme dadurch beeinflusst werden, welche Informationen bereitgestellt werden und wie diese dargeboten werden (Nelson und Merz 2002). Ein in erster Linie informeller Kommunikationsstil im Rahmen der Aufklärung führt beispielsweise häufiger zur Zustimmung an einer Studie. Bei einer Scheinstudie wurde hingegen gezeigt, dass die Bereitschaft an der Studie weiter teilzunehmen sinkt, wenn über erste Studienergebnisse aufgeklärt wird, die den Vorteil einer der Therapieoptionen gegenüber der anderen aufzeigen. ProbandInnen in Entwicklungsländern könnten anfälliger für Manipulation sein, da sie möglicherweise den ForscherInnen und ÄrztInnen, die sie rekrutieren, mehr Vertrauen schenken.

Darüber hinaus gibt es die Sorge, dass ProbandInnen in Entwicklungsländern möglicherweise studienrelevante Informationen nicht vollständig verstehen, was wiederum zu einem ethisch zweifelhaften informed consent führen würde. Diese Sorge ist darin begründet, dass ein niedriger sozioökonomischer Status häufig mit einem niedrigen Bildungsstand einhergeht (Denny und Grady 2006). 2009 gingen laut der Statistiken der UNESCO 89\% der Kinder in Entwicklungsländern in die Grundschule. In entwickelten Ländern waren es 97\%. In den meisten afrikanischen Ländern brechen über 30\% der Kinder die Grundschule vorzeitig ab (Unesco Institute for Statistics 2011). In Ghana wurde eine Studie durchgeführt, um das Verstehen der ProbandInnen besser einschätzen zu können. Hierbei wurden Interviews mit 1971 Frauen im Alter von 15 bis 45 durchgeführt, die an einer Placebokontrollierten Vitamin A Ergänzungs-Studie teilnahmen. Nur 13\% der Probandinnen wussten, dass nicht alle Kapseln der Studie gleich waren (Hill et al. 2008). Das bedeutet, dass nur ein sehr geringer Teil der Studienteilnehmerinnen verstanden hatte, wie eine Placebo-kontrollierte Studie abläuft und dass manche einen Wirkstoff und andere ein Placebo erhalten würden. Die Frauen hatten entweder keine Schulbildung oder hatten nur die Grundschule abgeschlossen (Hill et al. 2008). Flory erläutert, dass mehrere Studien nachweisen, dass StudienteilnehmerInnen mit einem höheren Bildungsstand oder Leselevel studienrelevante Informationen signifikant besser verstehen (Flory und Emanuel 2004).

Zudem gibt es einhergehend mit der Zunahme transnationaler Forschung Tendenzen ProbandInnen weniger Informationen bereitzustellen, da die Annahme besteht, diese können nicht vollständig verstanden werden (Kottow 2004). Kottow erläutert, dies sei bedingt durch die Vermutung, dass Armut und fehlender Zugang zu Bildung die Unfähigkeit autonom zu handeln und Entscheidungen zu treffen mit sich bringen würden (Kottow 2004). Hierdurch kommen Zweifel an der Qualität des informed consent auf, denn ProbandInnen, die ungenügend informiert werden, würden eventuell, wären sie vollständig aufgeklärt, eine andere Entscheidung treffen.

Außerdem kann die Zustimmung von ProbandInnen durch kulturelle Gegebenheiten beeinflusst werden. In vielen Entwicklungsländern besteht kulturell bedingt ein starkes Vertrauen in 
Forschungsinstitutionen und eine Tradition, das Treffen von Entscheidungen bevorzugt auf andere zu verlagern (Hill et al. 2008). Zusätzlich gibt es kulturell bedingt unterschiedliche Auffassungen des informed consent. Zum einen steht die Zustimmung des Individuums im Vordergrund (sog. individual informed consent). Zum anderen wird die Zustimmung der Familie oder Gemeinschaft in den Fokus gerückt (sog. family oder community informed consent) (Braune et al. 2008). Auf der einen Seite gibt es die Sorge, dass der informed consent als Instrument der Selbstbestimmung der ProbandInnen unzureichend sein könnte, wenn die Familie oder Gemeinschaft zu stark in die Entscheidungsfindung miteinbezogen wird. Dies könnte beispielsweise in Kulturen der Fall sein, die der Familie einen größeren Stellenwert zuschreiben als dem Individuum. Auf der anderen Seite kann ein family informed consent aus kulturellen Gründen auch als notwendig erachtet werden (Braune et al. 2008).

Da Ausbeutung als eine der Hauptsorgen im Rahmen medizinischer Studien in Entwicklungsländern gilt, ist es an dieser Stelle wichtig, den Zusammenhang zwischen Ausbeutung und informed consent genauer zu beleuchten. Ist es möglich, dass alle Elemente des informed consent hinreichend sind und ProbandInnen trotzdem ausgebeutet werden? Ein ungültiger informed consent, zum Beispiel durch Manipulation, stellt laut Wertheimer keine Bedingung für Ausbeutung dar (Wertheimer 1996, S. 247ff). Deswegen sei es wichtig zwischen Ausbeutung zu unterscheiden, die consensual und nonconsensual ist (Wertheimer 1996, S. 253). Hierbei bedeute consensual, dass der Ausgebeutete der Transaktion freiwillig zustimmt und nonconsensual, dass die Zustimmung beispielsweise durch Zwang oder Manipulation unzureichend ist. In beiden Fällen handele es sich um Ausbeutung (Wertheimer 1996). Dies bedeutet, ein gültiger informed consent kann Ausbeutung nicht verhindern, da es möglich ist, dass eine Person freiwillig in eine ausbeutende Transaktion einwilligt. Dies kann der Fall sein, wenn eine Transaktion für den Ausgebeuteten von Nutzen ist und es, rational betrachtet, sinnvoll ist zuzustimmen (Wertheimer 1996). Es wäre zum Beispiel möglich, dass ProbandInnen in Entwicklungsländern freiwillig in eine Studienteilnahme einwilligen, weil dies in ihrer Situation eine gute Option darstellt, um Zugang zu medizinischer Versorgung zu erhalten. Falls der Nutzen jedoch unfair ist, zum Beispiel aufgrund eines wesentlich höheren Nutzens auf Seiten der Pharmafirma und eines relativ hohen Risikos auf Seiten der ProbandInnen, würden die ProbandInnen trotz des hinreichenden informed consent ausgebeutet. Hierdurch zeigt sich abermals, dass Vulnerabilität im Kontext transnationaler Biomedizin nicht ausschließlich mit informed consent verknüpft werden sollte, sondern unter anderem die Anfälligkeit für Ausbeutung als ein Kriterium für Vulnerabilität notwendig ist.

Betrachtet man die oben aufgezeigten Konflikte, die bei Forschung in Entwicklungsländern auftreten können, wird deutlich, dass diese für die Aufstellung der Kriterien von Vulnerabilität mitberücksichtigt werden müssen. Im folgenden Kapitel sollen daher die aus den Konflikten herausgearbeiteten ethisch relevanten Aspekte im Zuge der Aufstellung der Kriterien für Vulnerabilität angewendet werden. 


\subsection{Vulnerabilitätskriterien für den Kontext medizinischer Forschung mit Frauen in}

Entwicklungsländern

Nach der Betrachtung verschiedener Definitionsmöglichkeiten für Vulnerabilität und zentraler ethischer Konflikte im Rahmen von Forschung mit Frauen und Forschung mit Menschen in Entwicklungsländern komme ich zu der Arbeitshypothese, dass für diese Arbeit eine Kombination der in Kapitel 3.1 bearbeiteten Definitionen sinnvoll ist. Da zuvor aufgezeigt wurde, dass weder eine rein kontextbezogene noch eine rein personenbezogene Definition ausreicht, sollen sowohl der Kontext der Studie als auch die Eigenschaften und die Lebenssituation der ProbandInnen als Vulnerabilitätskriterien berücksichtigt werden.

Für die noch folgende Analyse von zwei Fallstudien, möchte ich daher folgende Vulnerabilitätskriterien, die im folgenden Abschnitt noch genauer erläutert werden, anwenden und auf Plausibilität prüfen:

\section{Abb. 1: Vulnerabilitätskriterien}

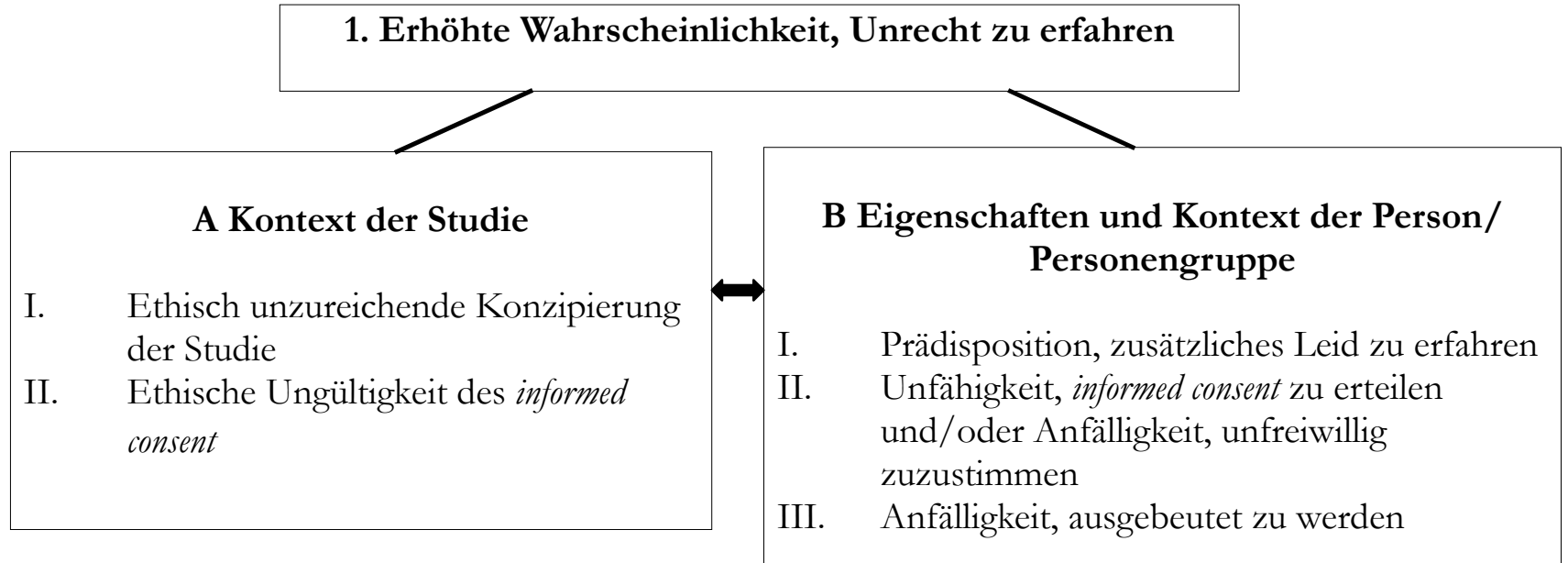

Als Grundlage für diese Vulnerabilitätskriterien dient Hursts Ansatz (Hurst 2008). Folglich ist das erste Vulnerabilitätskriterium, das gegeben sein muss, eine erböbte Wabrscheinlichkeit, Unrecht zu erfahren. Hieraus ergibt sich das Anrecht auf besonderen Schutz für vulnerable Personen oder Personengruppen. Um konkretisieren zu können, unter welchen Umständen und bei wem eine erhöhte Wahrscheinlichkeit Unrecht zu erfahren, zutreffend ist, sollen zusätzliche Vulnerabilitätskriterien angewendet werden. Diese sind aufgeteilt in Vulnerabilitätskriterien, die aus dem Kontext der Studie resultieren oder solche, die durch Eigenschaften und Kontext der Person/Personengruppe bedingt sind.

Vulnerabilitätskriterien, die unter den Kontext der Studie fallen, beinhalten Aspekte, die durch die Studie oder die ForscherInnen eine mögliche Vulnerabilität der ProbandInnen bedingen. Hierzu zählt unter 
anderem, ob die Studie aus ethischer Sicht sinnvoll konzipiert ist. Rein Fremdnützige Forschung in Entwicklungsländern stellt ein Beispiel für eine ethisch unzureichende Konzipierung einer Studie dar, und dies gilt es, im Rahmen der Analyse der Fallstudien zu überprüfen. Außerdem führt ein ungültiger informed consent von Seiten der ForscherInnen, zum Beispiel durch Manipulation, zu Vulnerabilität. Manche Individuen können je nach Rahmenbedingung einer Studie vulnerabel sein oder eben nicht (Levine et al. 2004). Deswegen kann Vulnerabilität auch durch das ausschließliche Zutreffen eines der Vulnerabilitätskriterien, die zu Kontext der Studie zählen, gegeben sein.

Die Vulnerabilitätskriterien, die zu Eigenschaften und Kontext der Person/Personengruppe zählen, fassen nicht nur Eigenschaften einer Person im engeren Sinne zusammen, sondern berücksichtigen auch die Lebenssituation von ProbandInnen. Die Unfähigkeit bestimmte Informationen zu verstehen, ist ein Beispiel für eine Eigenschaft von Personen, die zu Vulnerabilität führen könnte. Aspekte, die die Lebenssituation von ProbandInnen betrifft und ursächlich für Vulnerabilität sein könnten, sind beispielsweise eine prekäre finanzielle Lage oder ein niedriger Bildungsstand. Das erste zu Eigenschaften und Kontext der Person/Personengruppe gehörige Vulnerabilitätskriterium, gründet sich auf die Herangehensweise von Kottow. Vulnerable, laut Kottow als anfällig bezeichnete, Individuen haben schon Leid erfahren und sind somit prädisponiert zusätzliches Leid zu erfahren (Kottow 2003). Hierzu zählen Personen, die physisches oder psychisches Leid erfahren haben sowie Personen, die zum Beispiel aufgrund einer schwierigen finanziellen Lage prädisponiert sind, zusätzliches Leid zu erfahren. Das Vulnerabilitätskriterium Prädisposition, zusätəliches Leid zu erfahren ist für den Kontext transnationaler Studien mit Frauen von großer Bedeutung, da, wie bereits in Kapitel 3.2. und 3.3 dargestellt, die Wahrscheinlichkeit erhöht ist, dass Probandinnen in Entwicklungsländern bereits Leid in irgendeiner Form erfahren haben. Es muss jedoch für die jeweilige Studie stets kontextabhängig überprüft werden, ob dies auch der Fall ist, um voreilige Schlüsse zu vermeiden.

Die Unfähigkeit, informed consent zu erteilen und/oder Anfälligkeit, unfreinillig zuzustimmen ist das zweite Vulnerabilitätskriterium, das zu Eigenschaften und Kontext einer Person/Personengruppe zählt. Im Gegensatz zum ungültigen informed consent bedingt durch die ForscherInnen, wie bereits bei Kontext der Studie erläutert, bezieht sich dieses Vulnerabilitätskriterium auf einen ethisch unzureichenden informed consent bedingt durch die ProbandInnen und deren Lebenssituation. Hierzu zählen Personen, die nicht kompetent sind, die Entscheidung für oder wider eine Studienteilnahme zu treffen, die studienrelevante Informationen nicht verstehen können sowie Personen, die mit einer erhöhten Wahrscheinlichkeit unfreiwillig zustimmen. Im Kontext internationaler Forschung mit Frauen ist dies insofern von großer Relevanz, da Probandinnen möglicherweise kaum mit dem Konzept einer medizinischen Studie vertraut sind. Hierdurch kann ihre Verständniskompetenz eingeschränkt sein. Des Weiteren könnte das Verstehen studienrelevanter Informationen aufgrund eines niedrigen Bildungsstands begrenzt sein. Zusätzlich besteht die Sorge Probandinnen in Entwicklungsländern könnten unfreiwillig an einer Studie 
teilnehmen, da sie möglicherweise nur zustimmen, um Zugang zu medizinischer Versorgung zu erhalten. Das dritte Vulnerabilitätskriterium, das zu Eigenschaften und Kontext einer Person/Personengruppe zählt, ist die Anfälligkeit, ausgebeutet zu werden. Hier ist die Herangehensweise von Macklin besonders fruchtbar. Personen, die in Armut leben, sich in einer machtlosen Position befinden und einen niedrigen Bildungsstand haben, sind anfällig dafür, ausgebeutet zu werden und deswegen vulnerabel (Macklin 2003). Folglich spielt die Lebenssituation von ProbandInnen für die Überprüfung dieses Vulnerabilitätskriterium eine große Rolle. Dies ist wohl für die Betrachtung einer möglichen Vulnerabilität von Frauen in Indien im Kontext transnationaler Biomedizin eines der wichtigsten Vulnerabilitätskriterien. Sie begründet die verbreitete bioethische Sorge einer möglichen Ausbeutung von ProbandInnen bei Forschung in Entwicklungsländern. Allerdings hängt die schlussendliche Beurteilung, ob Ausbeutung im Rahmen einer Studienteilnahme stattfindet, davon ab, ob ein fairer Nutzen für die ProbandInnen besteht. Ein fairer Nutzen wiederum hängt von der Konzipierung der Studie ab und zählt folglich zu Kontext der Studie. Das Vulnerabilitätskriterium Anfälligkeit, ausgebeutet zu werden dient demnach zur Einschätzung, ob bestimmte ProbandInnen aufgrund ihrer aktuellen Lebensbedingungen mit einer erhöhten Wahrscheinlichkeit einem unfairen Nutzen zustimmen könnten. Es bedeutet jedoch nicht gleichzeitig, dass Ausbeutung tatsächlich stattfindet.

Es ist wichtig zu erwähnen, dass sich die oben genannten Vulnerabilitätskriterien teilweise überlappen können. Eine erhöhte Anfälligkeit, ausgebeutet zu werden oder zusätzliches Leid zu erfahren, könnte zum Beispiel in beiden Fällen durch Armut entstehen. Auch ist es möglich, dass eine Kombination von Vulnerabilitätskriterien aus Kontext der Studie und Eigenschaften und Kontext der Person/Personengruppen vorliegt. Eine Person könnte zum Beispiel bei einer Studie mit einem relativ hohen Risiko nicht kompetent sein zuzustimmen, bei einer Studie mit minimalem Risiko jedoch durchaus. Eine mögliche Kombination oder gar Potenzierung der verschiedenen Vulnerabilitätskriterien zeigt, dass Personen nicht immer einfach nur so vulnerabel sind, sondern dass Vulnerabilität durch bestimmte Kontexte entstehen kann, entweder bezüglich der Studie oder äußerer Umstände, die die Person selbst betreffen. Die Anwendung der Vulnerabilitätskriterien erlaubt zunächst vulnerable von nicht vulnerablen Personen zu unterschieden. Wenn ein oder mehrere Vulnerabilitätskriterien beim Überprüfen einer Studie beispielsweise zutreffen, dann ist eine Person oder Personengruppe vulnerabel. Trifft keines der Vulnerabilitätskriterien zu, ist die Person oder Personengruppe hingegen nicht vulnerabel. Lässt sich bei einer Person oder Personengruppe im Rahmen einer Studienteilnahme Vulnerabilität feststellen, kann das Zutreffen vieler Vulnerabilitätskriterien Aussagen über eine stark ausgeprägte Vulnerabilität zulassen. Folglich beruht die Anwendung der Vulnerabilitätskriterien in dieser Arbeit auf dem Verständnis eines graduellen Konzepts von Vulnerabilität. 


\section{Rechtlicher und sozialer Hintergrund}

Auf die Kontextualisierung des Vulnerabilitätskonzepts bezüglich transnationaler Forschung mit Frauen und der Erarbeitung der Vulnerabilitätskriterien für eigens diesen Kontext folgen in diesem Kapitel theoretische Hintergrundinformationen, die für die Analyse und Interpretation der Fallstudien notwendig sind.

Zum einen sollen internationale Ethikrichtlinien bezüglich Vulnerabilität im Kontext transnationaler Biomedizin mit Frauen untersucht werden, um hierdurch eine theoretische Grundlage der rechtlichen Aspekte für die Fallstudien zu schaffen (Kapitel 4.1). Zum anderen gilt es die Fallstudien in den rechtlichen und sozialen Kontext von medizinischen Studien in Indien einzubetten (Kapitel 4.2).

\subsection{Vulnerabilität, medizinische Forschung mit Frauen und transnationale Biomedizin in} internationalen Ethikrichtlinien

Da transnationale medizinische Forschung enormes ethisches Konfliktpotenzial birgt und es in der Vergangenheit Kontroversen über Verstöße ethischer Richtlinien im Zuge medizinischer Forschung in Entwicklungsländern gab, ist es für diese Arbeit unabdingbar internationale Ethikrichtlinien genauer zu beleuchten (Lurie und Wolfe 1997). Im folgenden Unterkapitel sollen daher die drei wichtigsten internationalen Ethikrichtlinien im Hinblick auf Vulnerabilität, Forschung mit Frauen und ethische Konflikte im Kontext transnationaler Biomedizin exemplarisch analysiert und abschließend verglichen werden. Die zu untersuchenden Richtlinien sind: Die Deklaration von Helsinki des Weltärztebundes (World Medical Association: WMA), die International Ethical Guidelines for Biomedical Research Involving Human Subjects des Council for International Organizations of Medical Sciences (CIOMS) und die Universal Declaration on Bioethics and Human Rights der United Nations Educational, Scientific and Cultural Organization (UNESCO). Die Deklaration von Helsinki und die Richtlinien der CIOMS wenden sich hauptsächlich an ÄrztInnen und ForscherInnen. Die Grundsätze der UNESCO hingegen adressieren vor allem Staaten, insbesondere auch Entwicklungsländer, um eine universelle Bioethik auf juristischer und politischer Ebene weiterzuentwickeln. Hierdurch begründet sich auch die Notwendigkeit der Grundsätze der UNESCO zusätzlich zur Deklaration von Helsinki und den Richtlinien der CIOMS (Langlois 2008). Zudem ist festzuhalten, dass alle drei Richtlinien sogenanntes soft law darstellen und somit keine unmittelbar rechtlich bindende Funktion haben (Langlois 2008; WMA 2013c). 


\subsubsection{WMA: Deklaration von Helsinki}

Der Weltärztebund wurde nach dem zweiten Weltkrieg 1947 mit den Zielen gegründet, die berufliche Unabhängigkeit von ÄrztInnen und ethische Standards in der Medizin sicherzustellen (WMA 2013b). Die Deklaration von Helsinki wurde vom Weltärztebund erstmals 1964 veröffentlicht und enthält ethische Grundsätze für medizinische Forschung am Menschen. Sie ist eines der wichtigsten Dokumente der Forschungsethik (Eckenwiler et al. 2008). In Deutschland prägte vor allem die Fassung aus dem Jahre 1975 entscheidend das Arzneimittelgesetz (Bundesärztekammer 2013). Trotz des weitreichenden Einflusses der Deklaration, stellt diese jedoch ein soft law dar und dient somit als Empfehlung (WMA 2013c). Die Deklaration wurde mehrfach verändert, zuletzt im Jahr 2013.

\section{$\underline{\text { Vulnerabilität }}$}

Im folgenden Abschnitt soll die Deklaration im Hinblick auf ihre Herangehensweise an das Konzept der Vulnerabilität genauer beleuchtet werden. Diesbezüglich gab es in der aktuellen Version der Deklaration von Helsinki (2013) bedeutende Änderungen im Vergleich zur vorherigen aus dem Jahre 2008. Wie bereits im Kapitel 3.1.1 erwähnt, verknüpft die Fassung von 2008 Vulnerabilität mit informed consent. So hieß es in Paragraph 9:

„[...] Some research populations are particularly vulnerable and need special protection. These include those who cannot give or refuse consent for themselves and those who may be vulnerable to coercion or undue influence" (WMA 2008, S. 2).

Somit stützte die Deklaration von Helsinki aus dem Jahre 2008 ihre Definition von Vulnerabilität auf die ethische Gültigkeit des informed consent. Auf der einen Seite stand die Kompetenz einer Person im Vordergrund und auf der anderen Seite die Freiwilligkeit des informed consent. Die aktuelle Version der Deklaration stellt das Thema Vulnerabilität insgesamt mehr in den Vordergrund, sie führt Vulnerable Groups and Individuals erstmals als eigene Überschrift auf. Des Weiteren rückt die Deklaration von Helsinki davon ab, Vulnerabilität mit informed consent zu verknüpfen. Die Änderungen der Deklaration von Helsinki bezüglich vulnerabler Gruppen zeigen die Aktualität und Wichtigkeit sich mit dem Konzept der Vulnerabilität in der Medizinethik auseinanderzusetzen. Die aktuelle Herangehensweise an Vulnerabilität im Paragraph 19 der Deklaration von Helsinki lautet folgendermaßen:

„Some groups and individuals are particularly vulnerable and may have an increased likelihood of being wronged or of incurring additional harm. All vulnerable groups and individuals should receive specifically considered protection" (WMA 2013a, S. 3). 
Zu Forschung mit vulnerablen Gruppen fordert die Deklaration in Paragraph 20 zudem folgendes:

„Medical research with a vulnerable group is only justified if the research is responsive to the health needs or priorities of this group and the research cannot be carried out in a non-vulnerable group. In addition, this group should stand to benefit from the knowledge, practices or interventions that result from the research" (WMA 2013a, S. 3).

Im Vergleich zur vorherigen Fassung steht nun eine erhöhte Wahrscheinlichkeit, Unrecht oder zusätzliches Leid zu erfahren im Vordergrund. Die Definition von Vulnerabilität über eine mögliche Unfähigkeit informed consent zu erteilen bleibt in der aktuellen Deklaration vollkommen unerwähnt. Hierdurch zeigt sich, dass die Deklaration von einer rein personenbezogenen Herangehensweise an Vulnerabilität Abstand nimmt und eine kontextbezogene Betrachtung ermöglicht. Eine Person oder Gruppe könnte nämlich in bestimmten Situationen eine erhöhte Wahrscheinlichkeit für Unrecht oder Leid aufweisen, in einer anderen Situation jedoch nicht. Zusätzlich wird der normative Aspekt des Konzepts der Vulnerabilität in der aktuellen Fassung der Deklaration verstärkt. Dies zeigt sich durch die Forderung, dass vulnerable Gruppen oder Personen spezifischen Schutz erhalten sollten. In der vorherigen Fassung wird lediglich erwähnt, dass vulnerable Personen besonderen Schutz benötigen, die Bereitstellung von Schutz jedoch nicht explizit gefordert. Darüber hinaus fordert die aktuelle Deklaration von Helsinki, dass nur mit vulnerablen Gruppen geforscht werden dürfe, wenn hierbei die Gesundheitsbedürfnisse dieser Gruppe adressiert würden und wenn das Forschungsprojekt nicht auch mit einer nicht-vulnerablen Gruppe realisierbar wäre. Die zuletzt genannte Bedingung ist neu in der Deklaration und könnte vor allem im Kontext transnationaler Biomedizin von Bedeutung sein. Der Paragraph 20 könnte nämlich als Versuch gewertet werden, sogenannte fremdnützige Forschung (siehe hierzu Kapitel 3.3) in Entwicklungsländern zu verhindern. Des Weiteren bestärkt die aktuelle Version der Deklaration, dass vulnerable Gruppen von Forschung, die mit ihnen durchgeführt wird, in irgendeiner Form profitieren muss. Die vorherige Fassung forderte lediglich, dass es wahrscheinlich sein solle, dass vulnerable Gruppen von Forschung profitieren.

\section{Forschung mit Frauen}

Ethische Konflikte oder mögliche Besonderheiten hinsichtlich medizinischer Forschung mit Frauen werden in der Deklaration von Helsinki nicht erwähnt.

\section{Transnationale Biomedizin}

Ethische Konflikte, die vor allem im Zusammenhang mit internationaler Forschung entstehen, werden in der Deklaration von Helsinki erst seit den Überarbeitungen im Jahr 2000 aufgegriffen (Macklin 2004, S. 2348). Diese sind: Paragraph 10, 16, 17, 18, 20 (bereits im Abschnitt Vulnerabilität erläutert), 23, 25 und 33 . 
Der Paragraph 10 in der aktuellen Version der Deklaration (2013) stellt einen Bezug zu transnationaler Biomedizin her. Dieser lautet:

„Physicians must consider the ethical, legal and regulatory norms and standards for research involving
human subjects in their own countries as well as applicable international norms and standards. No
national or international ethical, legal or regulatory requirement should reduce or eliminate any of the
protections for research subjects set forth in this Declaration” (WMA 2013a, S. 2). Hinsichtlich transnationaler Biomedizin zeigt sich, dass die Deklaration das Vorhandensein unterschiedlicher ethischer und rechtlicher Normen in unterschiedlichen Ländern zwar anerkennt, den Schutz von ProbandInnen mithilfe der Deklaration jedoch über diese Normen stellt und damit einen hohen normativen, da universellen Anspruch der Deklaration vertritt.

Paragraph 16, 17 und 18 greifen das Thema Risiken, Lasten und Nutzen im Rahmen medizinischer Forschung auf (WMA 2013a). Die Forderung die Risiken und Lasten auf der einen und den Nutzen eines Forschungsprojektes auf der anderen Seite gründlich abzuwägen, könnte als Versuch verstanden werden, Ausbeutung zu verhindern (WMA 2013a). Dies ist wiederum gerade bei internationaler Forschung von großer Bedeutung.

Hinsichtlich der Arbeit von Ethikkomitees legt die Deklaration in Paragraph 23 folgendes fest:

„[...] It (ethics committee) must take into consideration the laws and regulations of the country or countries in which the research is to be performed as well as applicable international norms and standards [...]" (WMA 2013a, S. 3).

Dieser Paragraph stellt zusätzlich einen Bezug zu transnationaler und vor allem auch multinationaler Forschung her und erkennt hierbei die wichtige Bedeutung der Ethikkomitees.

In Paragraph 25 geht die Deklaration indirekt auf mögliche kulturelle Unterschiede bezüglich des informed consent ein. Dieser lautet:

„Participation by individuals capable of giving informed consent as subjects in medical research must be voluntary. Although it may be appropriate to consult family members or community leaders, no individual capable of giving informed consent may be enrolled in a research study unless he or she freely agrees" (WMA 2013a, S. 4).

Demnach zeigt Paragraph 25, dass die Deklaration von Helsinki die Notwendigkeit eines sogenannten family/community informed consent für bestimmte Situationen anerkennt. Inwiefern je nach kulturellem Kontext ein family/community informed consent mehr oder weniger sinnvoll sein könnte, wird hingegen nicht explizit erwähnt (siehe hierzu auch Kapitel 3.3). Darüber hinaus fordert dieser Paragraph jedoch auch, dass keine Person für eine Studie rekrutiert werden dürfe, wenn diese nicht aus freien Stücken zustimmt (WMA 2013a). 
Ein vielfach diskutierter Konflikt, nämlich der Behandlungsstandard bei Placebo-kontrollierten Studien, wird in Paragraph 33 aufgegriffen. Hierzu heißt es, dass eine neue Maßnahme mit der gegenwärtig besten erwiesenen Maßnahme verglichen werden müsse. Die Verwendung eines Placebos bzw. keine Behandlung in einer Kontrollgruppe sei nur dann zulässig, wenn gegenwärtig keine erwiesene Maßnahme existiere oder die Verwendung eines Placebos bzw. keine Behandlung aus wissenschaftlichen Gründen zwingend notwendig sei und die betroffenen PatientInnen hierdurch kein erhöhtes Risiko für ernsten oder irreversiblen Schaden hätten (WMA 2013a). Insbesondere seit dem Bekanntwerden der bereits erwähnten unethischen Placebo-Studien zur HIV-Übertragung von Mutter auf Kind in Entwicklungsländern, wird diesem Paragraph eine große Bedeutung beigemessen (Macklin 2009).

Der Zugang zu nutzenbringenden Studieninterventionen für ProbandInnen nach Abschluss von Studien stellt einen weiteren Gegenstand für Debatten im Kontext transnationaler Biomedizin dar, der in der Deklaration von Helsinki in Paragraph 34 folgendermaßen aufgegriffen wird:

„In advance of a clinical trial, sponsors, researchers and host country governments should make provisions for post-trial access for all participants who still need an intervention identified as beneficial in the trial" (WMA 2013a, S. 5).

Die vorgestellten Paragraphen der Deklaration werden in Kapitel 4.1.4 mit den noch nachfolgenden Richtlinien der CIOMS und UNESCO verglichen. Im folgenden Kapitel sollen jedoch zunächst einzelne Punkte der Guidelines der CIOMS genauer erläutert werden.

\subsubsection{CIOMS: International Ethical Guidelines for Biomedical Research Involving Human Subjects}

Der Council for International Organizations of Medical Sciences (CIOMS) ist eine internationale nichtstaatliche Organisation, die eng mit der World Health Organization (WHO) und der UNESCO zusammenarbeitet. Die CIOMS veröffentlichte erstmals 1993 die International Ethical Guidelines for Biomedical Research Involving Human Subjects und zuletzt im Jahr 2002 eine aktualisierte Version (Macrae 2007; CIOMS 2002, S. 9). Ziel der Richtlinien der CIOMS ist es aufzuzeigen, wie die ethischen Standards der Deklaration von Helsinki insbesondere auch im Kontext medizinischer Forschung in Entwicklungsländern angewendet werden können (CIOMS 2002, S. 7ff). In Deutschland wird die CIOMS durch die Arbeitsgemeinschaft der Wissenschaftlichen Medizinischen Fachgesellschaften vertreten (Arbeitsgemeinschaft der Wissenschaftlichen Medizinischen Fachgesellschaften). 


\section{$\underline{\text { Vulnerabilität }}$}

Im folgenden Abschnitt soll die Verwendungsweise der CIOMS bezüglich Vulnerabilität dargelegt werden. Die Definition vulnerabler Personen der CIOMS lautet:

„Vulnerable persons are those who are relatively (or absolutely) incapable of protecting their own interests. More formally, they may have insufficient power, intelligence, education, resources, strength, or other needed attributes to protect their own interests" (CIOMS 2002, S. 64).

Diese Herangehensweise stützt sich insofern auf informed consent, als Personen durch eine selbstbestimmte Zustimmung oder Ablehnung ihre eigenen Interessen schützen könnten. Somit ist es plausibel, dass Personen, die dies nicht können, besondere Beachtung und besonderen Schutz benötigen. Die CIOMS fordert in Punkt 13, dass Forschung mit vulnerablen Individuen besonderer Rechtfertigung bedarf und die Rechte und das Wohl dieser Individuen besonders geschützt werden müssten. Ergänzend zu Punkt 13 zählt die CIOMS einige Beispiele vulnerabler Gruppen auf und nennt hierbei unter anderem Kinder, Medizinstudenten, ältere Menschen und schwer erkrankte Personen (CIOMS 2002, S. 64).

\section{Forschung mit Frauen}

Im Gegensatz zur Deklaration von Helsinki greift die CIOMS Besonderheiten von medizinischer Forschung mit Frauen auf. Die CIOMS legt in Punkt 16 der Richtlinien fest, dass Frauen im gebärfähigem Alter nicht von Studien ausgeschlossen werden dürften, da die Möglichkeit während einer Studie schwanger zu werden, nicht als Begründung dienen sollte eine Studienteilnahme einzuschränken. Sollte im Vorfeld schon bekannt sein, dass eine Studienteilnahme für eine schwangere Frau und ihren Fötus gefährlich sein könnte, müssten die ForscherInnen einen Schwangerschaftstest vor der Studie und sichere Verhütungsmittel während der Studienteilnahme bereitstellen. Könne dies jedoch aus rechtlichen oder religiösen Gründen nicht garantiert werden, sollten diese Frauen nicht rekrutiert werden (CIOMS 2002, S. 72). Im Kommentar zu Punkt 16 ergänzt die CIOMS, dass Frauen in den meisten Gesellschaften bezüglich einer Studienteilnahme diskriminiert würden und folglich das fehlende Wissen über die Sicherheit und Wirkung von Medikamenten für Frauen gefährlich sein könnte. Zusätzlich stelle der Ausschluss gebärfähiger Frauen von medizinischen Studien eine Einschränkung der Selbstbestimmung dieser Frauen dar. Die CIOMS fügt jedoch hinzu:

„,...] it must be acknowledged that in some parts of the world women are vulnerable to neglect or harm in research because of their social conditioning to submit to authority, to ask no questions, and to tolerate pain and suffering" (CIOMS 2002, S. 73).

Hier müssten ForscherInnen den Prozess des informed consent mit besonderer Vorsicht durchführen.

In keinem Fall dürfe das Einverständnis des Ehemanns oder Partners den informed consent der Studienteilnehmerin ersetzen (CIOMS 2002, S. 73). In Punkt 17 der Richtlinien geht die CIOMS auf 
Forschung mit Schwangeren ein und fordert, dass auch diese als potenzielle Studienteilnehmerinnen anerkannt werden. Allerdings sollten Schwangere nur an Studien teilnehmen, die für sie selbst oder ihren Fötus oder generell für schwangere Frauen von Nutzen sind (CIOMS 2002, S. 74). Die CIOMS ergänzt, dass die Entscheidung, ob die Risiken einer Studienteilnahme akzeptabel sind, bei der Mutter liege (CIOMS 2002, S. 74).

\section{Transnationale Biomedizin}

Da nahezu jeder Punkt der Richtlinien der CIOMS direkt oder indirekt Bezug auf ethische Konflikte im Rahmen internationaler Forschung nimmt, ist es im Rahmen dieser Arbeit nicht möglich alle diesbezüglichen Punkte detailliert zu erläutern.

Folgende Themenbereiche werden bezüglich transnationaler Biomedizin in den Richtlinien aufgegriffen (CIOMS 2002): Sogenannte doppelte ethische Standards (Punkt 3, S. 30), mögliche Notwendigkeit eines family/community informed consent aufgrund kultureller Gegebenheiten (Kommentar zu Punkt 4, S. 35), unangemessene Beeinflussung im Rahmen des informed consent (Punkt 7, S. 45), Nutzen-RisikoVerhältnis unter Einbeziehung des zukünftigen Nutzens für die Gesellschaft (Punkt 8, S. 47), Behandlungsstandard bei Placebo-kontrollierten Studien (Punkt 11, S. 54), Kompensation möglicher Schäden (Punkt 19, S. 78), Stärken lokaler wissenschaftlicher Infrastruktur und Ethikkomitees (Punkt 20, S. 80) und die Verpflichtung externer Sponsoren Behandlungsmöglichkeiten zur Verfügung zu stellen (Punkt 21, S. 81). Exemplarisch soll an dieser Stelle Punkt 10 Research in populations and communities with limited resources vorgestellt werden, da sich dieser Punkt direkt auf transnationale Forschung bezieht. Hier fordert die CIOMS, dass bei Forschungspopulationen mit eingeschränkten Ressourcen sichergestellt werden müsse, dass die Forschung auf die Gesundheitsbedürfnisse dieser Population ausgerichtet ist. Zudem müsse diese Forschungspopulation Zugang zu den durch die Forschung entstandenen Interventionen, Produkten oder erlangtem Wissen erhalten.

Im Kommentar zu Punkt 10 erläutert die CIOMS:

„This guideline is concerned with countries or communities in which resources are limited to extent that they are, or may be, vulnerable to exploitation by sponsors and investigators from the relatively wealthy countries and communities" (CIOMS 2002, S. 51).

Hierdurch zeigt sich, dass im Kontext transnationaler Biomedizin für die CIOMS die Hauptsorge die tatsächliche oder drohende Ausbeutung von ProbandInnen darstellt. 


\subsubsection{UNESCO: Universal Declaration on Bioethics and Human Rights}

Die United Nations Educational, Scientific and Cultural Organization (UNESCO) ist eine von 16 Sonderorganisationen der Vereinten Nationen und wurde 1945 mit dem Ziel gegründet die Zusammenarbeit zwischen den Völkern hinsichtlich Bildung, Wissenschaft und Kultur zu fördern und hiermit einen Beitrag zur Wahrung des Friedens zu leisten (UNESCO). Im Jahre 1993 gründete die UNESCO das International Bioethics Committee (IBC) und 1998 das Intergovernmental Bioethics Committee (IGBC) (German Commission for UNESCO). Auf der Grundlage eines Entwurfs des IBC wurde im Oktober 2005 die Universal Declaration on Bioethics and Human Rights fertiggestellt und von der Generalkonferenz der UNESCO angenommen (Andorno 2008). Alle Mitgliederstaaten der UNESCO, hierzu zählt auch Indien, verpflichteten sich die Grundsätze dieser Erklärung anzuwenden (UNESCO 2005a, S. 5). Wie bereits am Anfang des Kapitels erwähnt, stellt die Erklärung selbst ein rechtlich nicht verbindliches Instrument dar. Die angenommenen Grundsätze werden erst mithilfe nationaler Gesetze oder Regulierungen innerhalb der jeweiligen Mitgliedsstaaten verwirklicht (Langlois 2008). Deutschland, sowohl im ICB als auch IGB vertreten, war aktiv an der Erarbeitung der Bioethik-Erklärungen der UNESCO ${ }^{3}$ beteiligt (German Commission for UNESCO).

\section{$\underline{\text { Vulnerabilität }}$}

In der Erklärung wird Vulnerabilität an mehreren Stellen erwähnt. Es folgt allerdings keine Definition von Vulnerabilität. In der Einleitung zu den einzelnen Artikeln wird darauf hingewiesen, dass internationale Bioethik vor allem die besonderen Bedürfnisse der Entwicklungsländer, indigener Gemeinschaften und vulnerabler Bevölkerungsgruppen berücksichtigen müsse (UNESCO 2005b, S. 82). In Artikel 8 fordert die UNESCO:

„In applying and advancing scientific knowledge, medical practice and associated technologies, human vulnerability should be taken into account. Individuals and groups of special vulnerability should be protected and the personal integrity of such individuals respected“" (UNESCO 2005b, S. 56).

Hier wird zum einen auf eine allgemeine Vulnerabilität aller Menschen verwiesen, in dem die Schutzbedürftigkeit aller Menschen Berücksichtigung finden soll. Zum anderen wird eine besondere Vulnerabilität bestimmter Gruppen hervorgehoben, wobei jedoch zunächst keine Erläuterung folgt, wodurch sich diese besondere Vulnerabilität auszeichnet. Dies geschieht unter anderem indirekt in Artikel 5. Hier wird festgelegt, dass Personen, die nicht fähig sind, frei und selbständig zu entscheiden, besondere Maßnahmen zum Schutz ihrer Rechte und Interessen zustehen (UNESCO 2005b, S. 55).

\footnotetext{
${ }^{3}$ Hierzu zählen: Universal Declaration on the Human Genome and Human Rights (1997) International Declaration on Human Genetic Data (2003) Universal Declaration on Bioethics and Human Rights (2005)
} 
Des Weiteren wird in Artikel 7 besonderer Schutz für Nichteinwilligungsfähige gefordert (UNESCO 2005b, S. 55). Zusätzlich wird in Artikel 24 Solidarität gefordert und dies vor allem für vulnerable Personen oder Gruppen. Hierbei werden folgenden Gründe für Vulnerabilität genannt:

„States should respect and promote solidarity between and among States, as well as individuals, families, groups and communities, with special regard for those rendered vulnerable by disease or disability or other personal, societal or environmental conditions and those with the most limited resources" (UNESCO 2005b, S. 61).

Die Hervorhebung derjenigen, die die wenigsten Mittel zur Verfügung haben, kann als Bezugnahme auf Forschung mit Menschen in Entwicklungsländern interpretiert werden.

\section{Forschung mit Frauen}

In der Präambel der Erklärung wird darauf verwiesen, dass es bei der Beurteilung sozialer Gegebenheiten und Gleichheit wichtig sei auf die Stellung von Frauen zu achten (UNESCO 2005b, S. 53). Zudem wird in Artikel 14 darauf hingewiesen, dass durch den Fortschritt der Wissenschaft der Zugang zu medizinischer Versorgung und hochwertigen Medikamenten besonders für Frauen und Kinder gefördert werden soll. Spezifischere Aspekte hinsichtlich Forschung mit Frauen, beispielsweise der Umgang mit Schwangeren als potenzielle Studienteilnehmerinnen, werden in der Erklärung nicht aufgegriffen (UNESCO 2005b, S. 57).

\section{Transnationale Biomedizin}

Im folgenden Abschnitt gilt es, Artikel hervorzuheben, die ethische Konflikte im Kontext transnationaler Biomedizin betreffen. In Artikel 6 wird beispielsweise festgelegt, dass es in geeigneten Fällen möglich sei, die Zustimmung eines Rechtsvertreters einer Personengruppe oder Gemeinschaft einzuholen. Allerdings solle die Einwilligung einer Einzelperson hierdurch in keinem Fall ersetzt werden (UNESCO 2005b, S. 55). Zudem fordert die UNESCO in Artikel 12 die Berücksichtigung kultureller Vielfalt, wobei dies jedoch nicht dazu dienen dürfe, die Menschenwürde, die Menschenrechte oder die Grundsätze der Erklärung zu verletzen (UNESCO 2005b, S. 57). Des Weiteren soll die gesamte internationale Gemeinschaft, insbesondere die Entwicklungsländer, am Nutzen aus wissenschaftlicher Forschung teilhaben (Artikel 15) (UNESCO 2005b, S. 57f). Die UNESCO nennt hierzu unterschiedliche Formen von Nutzen, wie zum Beispiel Zugang zu hochwertiger medizinischer Versorgung oder Bereitstellen von neuen diagnostischen und therapeutischen Verfahren oder Produkten, die aus der Forschung stammen (UNESCO 2005b, S. 58). Artikel 21 der Erklärung legt Bedingungen für sogenannte Transnational practices fest. Eine dieser Bedingungen legt fest, dass Staaten, Institutionen und Fachleute, die mit grenzüberschreitenden Tätigkeiten befasst sind, diese Tätigkeiten im Einklang mit den Grundsätzen der Erklärung durchführen. Darüber hinaus soll eine angemessene 
ethische Bewertung von Forschung, auf der Grundlage der Grundsätze der Erklärung, von Seiten des Gaststaates und von Seiten des Staates stattfinden, in dem der Geldgeber ansässig ist. Zusätzlich soll gesundheitsbezogene Forschung auf die Bedürfnisse der Gaststaaten ausgerichtet sein (UNESCO 2005b, S. 59f). Ein angemessener Behandlungsstandard bezüglich Placebo-kontrollierter Studien bleibt in der Erklärung der UNESCO unerwähnt. Insgesamt lässt sich jedoch festhalten, dass die Grundsätze der UNESCO in zahlreichen Artikeln explizit oder implizit auf ethische Konflikte von Forschung in Entwicklungsländern eingehen.

\subsubsection{Internationale Ethikrichtlinien im Vergleich}

Nach der Betrachtung der einzelnen Richtlinien sollen diese nun bezüglich der untersuchten Aspekte exemplarisch miteinander verglichen werden. Alle drei Richtlinien zeigen jeweils eine unterschiedliche Herangehensweise an Vulnerabilität. Hierdurch lässt sich erneut folgern, dass keine einheitliche Definition von Vulnerabilität existiert. Als Gemeinsamkeit der Guidelines der CIOMS und der Grundsätze der UNESCO lässt sich jedoch festhalten, dass Vulnerabilität direkt oder indirekt jeweils mit informed consent verknüpft wird. Wie jedoch bereits in Kapitel 3.1.1 erläutert, reicht informed consent nicht aus, um Vulnerabilität definieren zu können. Die Deklaration von Helsinki weist hingegen in ihrer aktuellen Version (2013) einen Ansatz von Vulnerabilität unabhängig von informed consent auf und stellt somit eine neue Herangehensweise im Vergleich zu vorherigen Fassungen dar. In der Deklaration von Helsinki wird Vulnerabilität nämlich mit der erhöhten Wahrscheinlichkeit Unrecht oder zusätzliches Leid zu erfahren definiert. Im Gegensatz zur Deklaration von Helsinki werden in den Richtlinien der CIOMS und der Erklärung der UNESCO mögliche Ursachen für Vulnerabilität genannt und hierdurch ein weiter gefasstes Verständnis von Vulnerabilität festgelegt. Die CIOMS beschreibt zum Beispiel Personen mit eingeschränkter Macht, Intelligenz oder Bildung als vulnerabel. In der Erklärung der UNESCO wird beschrieben, dass Personen durch Krankheit, Behinderung oder beispielsweise gesellschaftliche Bedingungen vulnerabel werden. Die Deklaration von Helsinki und die Richtlinien der CIOMS zeigen eine kontextbezogene Herangehensweise an Vulnerabilität, da Personen je nach Kontext Unrecht oder zusätzliches Leid erfahren könnten beziehungsweise je nach Kontext fähig sind ihre Interessen zu schützen oder nicht. Da die UNESCO keine eigene Definition von Vulnerabilität gibt, kann nicht eindeutig eingeschätzt werden, ob deren Verwendung von Vulnerabilität in erster Linie personen- oder kontextbezogen ist. Die Beispiele, die die UNESCO für vulnerable Personen angibt, sind jedoch sowohl personen- als auch kontextbezogen. Zum einen sind Krankheit, Behinderung und gesellschaftliche Rahmenbedingungen Aspekte, die in dieser Arbeit als personenbezogen eingeteilt 
werden, da sie von der Person selbst oder deren Lebensbedingungen in die Situation einer Studienteilnahme eingebracht werden. Zum anderen zeigt jedoch die Formulierung, dass Personen vulnerabel werden im Gegensatz zu vulnerabel sind, dass die UNESCO Vulnerabilität auch im Sinne eines kontextbezogenen Konzepts versteht. Die Formulierung vulnerabel werden suggeriert, dass Personen in bestimmten Situationen, zum Beispiel im Kontext einer bestimmten Studienteilnahme, vulnerabel sein können, in einer anderen Situation, im Rahmen einer anderen Studie, jedoch nicht zwingend vulnerabel sind.

Hinsichtlich der in Kapitel 3.4 aufgestellten Vulnerabilitätskriterien lassen sich sowohl Gemeinsamkeiten als auch Unterschiede zu den drei erläuterten Richtlinien feststellen. Eine erhöhte Wahrscheinlichkeit Unrecht zu erfahren gilt in dieser Arbeit als Hauptvulnerabilitätskriterium und findet sich auch in der Deklaration von Helsinki wieder. Das zweite Kriterium, das in der Deklaration von Helsinki genannt wird, nämlich die erhöhte Wahrscheinlichkeit zusätzliches Leid zu erfahren, wird auch in dieser Arbeit angewendet, allerdings als Unterkriterium. Die Herangehensweise an Vulnerabilität der CIOMS im Sinne der Unfähigkeit die eigenen Interessen schützen zu können, wird in dieser Arbeit jedoch nicht explizit verwendet. Indirekt findet sich diese Herangehensweise aber bei der Verknüpfung von Vulnerabilität und informed consent wieder, die auch in den Vulnerabilitätskriterien dieser Arbeit aufgegriffen wird. Denn der informed consent ist ein Konzept, das den Schutz der eigenen Interessen ermöglicht. Eine indirekte Verknüpfung von Vulnerabilität und informed consent ist auch eine Gemeinsamkeit der Erklärung der UNESCO (siehe hierzu Kapitel 4.1.3) und der in Kapitel 3.4 vorgestellten Vulnerabilitätskriterien. Ein bedeutender Unterschied zwischen den drei Richtlinien im Vergleich mit den Vulnerabilitätskriterien dieser Arbeit ist der, dass keine der Richtlinien einen Zusammenhang von Vulnerabilität und Ausbeutung herstellt. Die Anfälligkeit, ausgebeutet zu werden, ist jedoch ein wichtiges Kriterium für Vulnerabilität im Kontext transnationaler Biomedizin und ist deswegen eines der Vulnerabilitätskriterien, das es anhand der Fallstudien zu überprüfen gilt.

Hinsichtlich spezifischer Faktoren, die Forschung mit Frauen betreffen, weisen die drei Richtlinien keine Gemeinsamkeiten auf. Die Deklaration von Helsinki bezieht zu Debatten bezüglich besonderer Aspekte bei Forschung mit Frauen keine Stellung. Die CIOMS hingegen nimmt sowohl die besondere Situation schwangerer als auch die gebärfähiger Frauen als Studienteilnehmerinnen in ihre Richtlinien auf und verweist zudem auf besondere soziale Aspekte in diesem Kontext. Die Erklärung der UNESCO fordert zwar die besondere Berücksichtigung der Stellung der Frau bei der Bewertung sozialer Verhältnisse, geht dann jedoch nicht genauer darauf ein und lässt Schwangerschaft oder Gebärfähigkeit im Kontext medizinischer Forschung unerwähnt. Das ist insofern verwunderlich, da bei Debatten um den Einschluss beziehungsweise Ausschluss von Frauen in medizinische Forschung üblicherweise Schwangerschaft oder Gebärfähigkeit im Vordergrund stehen.

Als Gemeinsamkeit lässt sich somit festhalten, dass alle drei Richtlinien direkt oder indirekt auf 
ethische Aspekte im Kontext transnationaler Biomedizin eingehen. Auffällig ist jedoch, dass die für Deutschland besonders relevante Deklaration von Helsinki jeweils nur indirekt auf ethische Konflikte im Rahmen von internationaler Forschung verweist. Entsprechende Paragraphen nennen Menschen in Entwicklungsländern als potenzielle ProbandInnen nicht explizit, sondern verwenden Umschreibungen wie beispielsweise benachteiligte oder vulnerable Bevölkerungsgruppen (Paragraph 17). Diese Umschreibungen könnten sich auf Menschen in Entwicklungsländern beziehen, könnten jedoch auch andere Forschungspopulationen meinen. Die Richtlinien der CIOMS hingegen wurden vor allem veröffentlicht, um die ethischen Standards der Deklaration von Helsinki auch im Kontext biomedizinischer Forschung in Entwicklungsländern anwendbar zu machen (CIOMS 2002, S. 11). Folglich stehen Aspekte, die Forschung in Entwicklungsländer betreffen im Vordergrund. Die CIOMS erläutert hierbei den Konflikt, inwiefern ethische Prinzipien als universal oder als kulturell relativ betrachtet werden können. Hierzu formuliert die CIOMS noch folgende Zielsetzung:

„[...] research involving human subjects must not violate any universally applicable ethical standards, but acknowledge that in superficial aspects, the application of the ethical principles, e.g., in relation to individual autonomy and informed consent, needs to take account of cultural values [...]" (CIOMS 2002, S. 11).

In der Erklärung der UNESCO stehen internationale Forschung und damit verbundene ethische Konflikte ebenfalls im Vordergrund. Der Präsident der Deutschen UNESCO-Kommission fordert in seinem Grußwort zur Erklärung, dass die Globalisierung und somit die Internationalisierung der Forschung eine Internationalisierung der Ethik nach sich ziehen müsse. Außerdem erläutert er, dass die Erklärung die Bioethik auf die Basis des allgemeingültigen Rahmens der Menschenrechte stelle (UNESCO 2005a, S. 7).

Nach der Analyse der Fallstudien soll in Kapitel 5.6 geprüft werden, inwiefern die vorgestellten Richtlinien hilfreich sein können, um Vulnerabilität im Rahmen transnationaler Biomedizin mit Frauen zu bewerten. 


\subsection{Medizinische Studien in Indien}

Die Anzahl klinischer Studien in Indien stieg in den letzten Jahren enorm an. Schätzungen zufolge wird aktuell eine von vier klinischen Studien weltweit in Indien durchgeführt (Sengupta 2009). 2011 waren in Indien über 1800 Studien registriert (Pandey et al. 2011). Gründe hierfür sind unter anderem geringere Kosten im Vergleich zu Forschung in Industriestaaten (bis zu 60\%, Sengupta 2009), hoher technischer Standard, gut ausgebildete und erfahrene ForscherInnen und Kommunikation sowie Daten in englischer Sprache. Zusätzlich bringt die große Population Indiens einige Vorteile für ForscherInnen, nämlich eine große genetische Vielfalt, hohe Einschreiberaten für Studien und viele sogenannte Treatment-naive ProbandInnen, also Menschen, die bisher keine medikamentöse Behandlung erhielten, wodurch sich eine geringe Wahrscheinlichkeit für Arzneimittelinteraktionen ergibt. Des Weiteren ist es möglich, in Indien eine große Spanne verschiedener Erkrankungen zu erforschen, also sowohl Erkrankungen, die mit Armut einhergehen als auch sogenannte lifestyle diseases (Sengupta 2009; George und Srinivasan 2006).

Um in Kapitel 5 Fallstudien im Hinblick auf mögliche Vulnerabilität von Probandinnen in Indien analysieren zu können, sollen zunächst allgemeine Rahmenbedingungen für medizinische Studien in Indien beleuchtet werden. Im folgenden Kapitel werden daher rechtliche Rahmenbedingungen sowie ethische Richtlinien bezüglich medizinischer Forschung in Indien dargestellt. Im darauffolgenden Kapitel folgen Erläuterungen hinsichtlich gesellschaftlicher Faktoren, die ethische Aspekte im Kontext transnationaler Forschung in Indien beeinflussen könnten. Mit Blick auf die zentrale Fragestellung der Arbeit stehen hierbei vor allem Frauen in Indien im Vordergrund.

\subsubsection{Rechtliche Rahmenbedingungen}

In Indien sind viele Institutionen an der Regulierung, Zulassung und Überwachung klinischer Studien beteiligt. Im folgenden Abschnitt sollen einige dieser Institutionen und deren Hauptfunktion vorgestellt werden. Zunächst muss das Ministry of Health and Family Welfare (MOHFW) erwähnt werden, das als Teil der indischen Regierung verschiedene Abteilungen enthält, wie beispielsweise das Department of Health Research (DHR). Dem Ministry of Health untersteht außerdem die Central Drug Standard Control Organization (CDSCO). Die CDSCO kontrolliert den Import von Arzneimitteln, die Zulassung neuer Wirkstoffe sowie die Genehmigung klinischer Studien (CDSCO, S. 1f). Zudem veröffentlichte der CDSCO die Good Clinical Practice Guidelines, die als ethischer und wissenschaftlicher Standard für die Planung und Durchführung von Forschungen am Menschen dienen sollen (CDSCO 2004). An dieser 
Stelle ist es wichtig zu erwähnen, dass die CDSCO laut der Bundeskoordination Internationalismus $(\mathrm{BUKO})^{4}$ personell als zu schwach besetzt gilt, um die hohe und vor allem weiter wachsende Anzahl von Studien in Indien kontrollieren zu können (BUKO 2010b). Jede klinische Studie, die in Indien begonnen wird, muss zuvor vom Drugs Controller General of India (DCGI) als Mitglied der CDSCO genehmigt werden (Srinivasan 2009). Dieser soll außerdem die Einhaltung ethischer Standards bei der Durchführung klinischer Studien überwachen (BUKO 2010b). Im Kontext klinischer Forschung in Indien muss zudem der Indian Council of Medical Research (ICMR) erwähnt werden, der durch das Department of Health Research finanziert wird. Zu den Aufgaben des ICMR zählen unter anderem die Koordination und die Förderung biomedizinischer Forschung (ICMR 2010). 2006 veröffentlichte der ICMR außerdem Ethical Guidelines for Biomedical Research on Human Participants (ICMR 2006). Zusätzlich wurde 2007 das Clinical Trials Registry India (CTRI) gegründet, das zur Registrierung klinischer Studien in Indien dient (Tharyan 2007). Die Richtlinien des ICMR sowie der Ablauf der Registrierung klinischer Studien in Indien werden noch im Laufe dieses Kapitels genauer erläutert.

Klinische Studien in Indien werden rechtlich durch den Schedule $Y$ der Drugs and Cosmetics Rules des Department of Health reguliert. Dieser enthält unter anderem Regulierungen zur Zulassung klinischer Studien, zur Verantwortung der SponsorInnen und ForscherInnen, Voraussetzungen für den informed consent sowie zu den Aufgaben der Ethikkomitees (MOHFW 2005). In den letzten Jahren ergaben sich einige bedeutende Änderungen des Schedule Y. Vor 2005 beispielsweise durften Wirkstoffe, die im Ausland entwickelt wurden, nur in Indien getestet werden, wenn die vorherige Phase einer Studie, die im Ausland bereits abgeschlossen war, in Indien wiederholt wurde. Wurde zum Beispiel eine Studie im Ausland in Phase III abgeschlossen, musste in Indien erneut mit Phase II begonnen werden (Sengupta 2009). Seit 2005 ist es allerdings möglich, Studien, nachdem Phase I im Ausland abgeschlossen wurde, in Indien direkt mit der nächsten Phase fortzuführen (MOHFW 2005, S. 503). Eine Wiederholung vorheriger Studienphasen ist somit nicht mehr zwingend notwendig und dadurch könnte Indien als Zielort für klinische Studien noch mehr favorisiert werden. Eine mögliche Hürde für externe Pharmafirmen, Studien in Indien durchzuführen, nämlich Studienphasen wiederholen zu müssen und somit aus finanzieller und zeitlicher Sicht mehr investieren zu müssen, ist seit 2005 deutlich reduziert. Die Gesetzesänderung könnte allerdings auch aus ethischer Sicht als positiv gewertet werden, da die Wiederholung einer bereits abgeschlossenen Studienphase vermutlich keinen neuen Erkenntniswert bringt und somit die ProbandInnen unnötigen Risiken oder Unannehmlichkeiten aussetzt.

Des Weiteren ist es erst seit 2005 verpflichtend, dass die Dokumente einer Studie durch ein Ethikkomitee geprüft werden. Zuvor war dies nur eine Empfehlung (Srinivasan 2009). Anfang des Jahres 2013 wurden weitere bedeutende Veränderungen des Schedule Y vorgenommen. Dies geschah als

4 Pharma-Kampagne mit dem Ziel die Aktivitäten der deutschen Pharmaindustrie in der Dritten Welt zu untersuchen (http://www.bukopharma.de/) 
Reaktion auf eine eidesstattliche Erklärung des Deputy Drugs Controller beim Obersten Gerichtshof, die das Scheitern der CDSCO beim Schutz der Rechte von StudienteilnehmerInnen aufzeigt (Singh 2013; Jesani 2013). Die Erklärung legt dar, dass von Beginn 2005 bis Mitte 2012 in 475 Studien 14.616 schwerwiegende unerwünschte Ereignisse, sogenannte serious adverse events (SAE), gemeldet wurden davon 2.644 Todesfälle (18\%). Bei nur 80 (3\%) der Todesfälle wurden diese als ursächlich oder in Zusammenhang mit der Studie stehend bewertet und nur der Hälfte der Familien der Opfer wurde eine Entschädigung gezahlt (Singh 2013; Jesani 2013). Eine der Änderungen des Schedule Y besagt demnach, dass im Falle einer Verletzung eines/r Studienteilnehmers/in diese Person kostenfrei medizinisch versorgt werden soll (MOHFW 2013b, S. 9). Hierbei ist bemerkenswert, dass diese Forderung nicht voraussetzt, dass eine Erkrankung mit der Studie oder dem zu testenden Medikament zusammenhängen muss (Jesani 2013). Des Weiteren wird in der aktuellen Fassung des Schedule Y (2013) festgelegt, dass StudienteilnehmerInnen oder von ihnen benannte Personen eine Entschädigung für eine mit der Studienteilnahme assoziierte Verletzung oder Todesfall erhalten, und diese müsse über den Kosten der medizinischen Behandlung liegen (MOHFW 2013b, S. 9). Es werden sieben Kriterien aufgeführt, die festlegen, ob eine Verletzung oder ein Todesfall als mit der Studienteilnahme assoziiert gelten sollte. Hierbei werden unter anderem auch die Wirkung von Placebos oder der fehlende therapeutische Effekt bei einem sich als nicht wirksam herausstellenden Medikament miteinbezogen (MOHFW 2013b, S. 9). Folglich werden mögliche Risiken für alle StudienteilnehmerInnen berücksichtigt, nämlich auch für Personen, die der Kontrollgruppe zugeordnet wurden. Aus ethischer Sicht sind diese Änderungen positiv zu bewerten, da die Rechte der ProbandInnen als Priorität gelten. Eine weitere Änderung des Schedule $Y$ betrifft das Einrichten eines Expertenausschusses zur Beurteilung von studienassoziierten Todesfällen (DCGI 2013). Jesani kritisiert, dass die CDSCO sowohl finanziell, personell als auch fachlich zu wenig Ressourcen aufweise, um den Zusammenhang zwischen Todesfällen und Studienteilnahmen adäquat beurteilen zu können. Demnach würden weitere Ausschüsse oder Komitees solche Beurteilungen nur verzögern (Jesani 2013). Im Gegensatz zum Schedule Y aus dem Jahre 2005 müssen sich zudem seit der Gesetzesänderung 2013 alle Ethikkomitees bei der CDSCO registrieren (MOHFW 2013a, S. 8). Dies stellt einen ersten Schritt für die Überprüfung der Arbeit der Ethikkomitees dar. Seit 2005 fordert der Schedule Y außerdem, dass eine Studie nur nach Zustimmung durch ein Ethikkomitee begonnen werden darf (MOHFW 2005, S. 504). Es wird zudem erläutert, dass Ethikkomitees insbesondere darauf achten sollten, die Rechte, die Sicherheit und das Wohl von vulnerablen Gruppen zu schützen. Hier werden als Beispiele unter anderem folgende Gruppen aufgezählt: Personen innerhalb hierarchischer Strukturen (Gefängnisinsassen, Medizinstudenten usw.), Patienten mit unheilbaren Erkrankungen, arbeitslose oder verarmte Personen, Patienten in Notsituationen, ethnische Minderheiten (MOHFW 2005, S. 506). Frauen als vulnerable Gruppe werden nicht genannt. 
Außerdem solle im Studienbericht dokumentiert werden, dass die Studie in Übereinstimmung mit den ethischen Prinzipien der Deklaration von Helsinki, den Good Clinical Practice Guidelines der CDSCO und den Ethical Guidelines for Biomedical Research on Human Participants des ICMR durchgeführt wurde (Tharyan 2007; MOHFW 2005, S. 516). Letztere wurden 2006 vom ICMR veröffentlicht und versuchen sich der Herausforderung zu stellen, universale ethische Prinzipien im Kontext biomedizinischer Forschung in der multikulturellen Gesellschaft Indiens anwendbar zu machen (ICMR 2006, S. 1). Im Zusammenhang mit der Auswahl besonderer Gruppen als ProbandInnen werden in den Guidelines vulnerable Gruppen erwähnt. Hierbei werden schwangere und stillende Frauen sowie Kinder als besondere Gruppen einzeln aufgeführt, jedoch nicht den vulnerablen Gruppen zugeteilt, wobei dies nicht begründet wird. In den Guidelines findet sich keine Definition von Vulnerabilität, sondern vielmehr Forderungen zum Umgang mit bestimmten Gruppen. Diese lauten folgendermaßen:

„Research on genetics should not lead to racial inequalities; persons who are economically or socially
disadvantaged should not be used to benefit those who are better off than them; rights and welfare of
mentally challenged and mentally differently able persons who are incapable of giving informed consent
or those with behavioral disorders must be protected. [...]; adequate justification is required for the
involvement of participanty such as prisoners, students, subordinates, employees, service personnel etc.
who have reduced autonomy as research participants, since the consent provided may be under duress or
various other compelling reasons“ (ICMR 2006, S. 28f). Es zeigt sich also, dass in den Guidelines Vulnerabilität mit verschiedenen Attributen verknüpft wird. Zum einen stehen ethnische und sozioökonomische Gegebenheiten von Personen im Vordergrund. Zum anderen wird Vulnerabilität mit der Unfähigkeit, informed consent zu erteilen oder unfreiwillig zuzustimmen, verknüpft. Einzelne Abschnitte der Guidelines können als Bezug auf transnationale Forschung verstanden werden. Es werden beispielsweise die Prinzipien der Nicht-Ausbeutung, Principles of non-exploitation, genauer beschrieben. Als generelle Regelung gilt hierbei, dass StudienteilnehmerInnen für ihre Mitwirkung an Forschung entschädigt werden sollen. Zudem fordern diese Prinzipien folgendes: „Such human participants should be selected so that the burdens and benefits of the research are distributed without arbitrariness, discrimination or caprice“ (ICMR 2006, S. 5). Hierbei werden verschiedene Formen des Nutzens für StudienteilnehmerInnen festgelegt, wie zum Beispiel Krankenversicherung oder medizinische Versorgung während einer Studie (ICMR 2006, S. 5). Im Vergleich mit den in Kapitel 4.1 untersuchten Richtlinien zeigt sich, dass die Guidelines des ICMR ein relativ weit gefasstes Konzept von Vulnerabilität vertreten und Vulnerabilität nicht ausschließlich mit informed consent verknüpfen. Des Weiteren ist bemerkenswert, dass es sowohl in der Deklaration von Helsinki als auch in den Richtlinien der CIOMS und UNESCO keine so explizit formulierten Prinzipien zur Nicht-Ausbeutung gibt. Inhaltlich decken sich die Prinzipien der Nicht-Ausbeutung allerdings teilweise mit den Paragraphen 16, 17 und 18 der Deklaration von Helsinki (WMA 2013), Punkt 10 und 12 der Richtlinien der CIOMS (CIOMS 2002) und Artikel 4 der Richtlinien der 
UNESCO (UNESCO 2005).

Nach der Betrachtung theoretischer Aspekte bezüglich der Durchführung klinischer Studien in Indien soll nun auch die praktische Umsetzung der Gesetze genauer beleuchtet werden. Hierbei stellt insbesondere die Regulierung und Kontrolle von Ethikkomitees ein großes Problem dar, denn über die momentane Arbeitsweise der Ethikkomitees sind kaum Daten und Informationen verfügbar (George 2011; Taur et al. 2011). In einer von Taur et al. 2011 veröffentlichten Studie über Ethikkomitees in Indien werden jedoch ernsthafte Mängel festgestellt. $20 \%$ der in der Studie überprüften Zulassungsdokumente der Ethikkomitees für klinische Studien enthielten entgegen der Vorschrift des Schedule Y keine Auflistung der Namen der TeilnehmerInnen von der entsprechenden Ethikkomiteesitzung. Außerdem hatten vier Ethikkomitees über die Genehmigung einer Studie abgestimmt, obwohl die TeilnehmerInnenanzahl der Sitzung nicht der vorgeschriebenen Mindestanzahl von fünf TeilnehmerInnen entsprach. Des Weiteren fehlte in den meisten Dokumenten die Erwähnung über die Anwesenheit einer Person mit juristischem, sozialwissenschaftlichem oder bioethischem Hintergrund, wobei dies bei Entscheidungsfindungen in Ethikkomitees laut Taur et al. äußerst wichtig wäre. Kritisch wird von den AutorInnen auch die mangelnde Bereitschaft der Ethikkomitees gesehen, an einer solchen Evaluationsstudie teilzunehmen (nur ein Drittel der angefragten Ethikkomitees stimmte zu). Da die Studie mit einer kleinen Fallzahl durchgeführt wurde, bleibt unklar, ob die Mängel, die bei der Arbeitsweise der an der Studie teilnehmenden Komitees festgestellt wurden, Einzelfälle darstellen oder inwiefern die Arbeitsweise von Ethikomitees in Indien generell als problematisch einzuschätzen ist (Taur et al. 2011).

Der Ablauf der Registrierung klinischer Studien in Indien soll genauer erörtert werden, da auch er die Komplexität der Situation verdeutlicht. Wie bereits erwähnt, können seit 2007 Studien im Clinical Trials Registry-India (CTRI) registriert werden. Hierdurch soll Transparenz, Verantwortung bzw. Haftung und Zugänglichkeit hinsichtlich klinischer Studien hergestellt werden (CTRI). Das Registrieren einer Studie in Indien war zunächst freiwillig (Tharyan 2007). Seit 2009 ist dies jedoch durch den Drugs Controller General India (DCGI) verpflichtend geworden (CTRI). Außerdem ist die Registrierung von Studien auch durch die Deklaration von Helsinki (Paragraph 35) vorgeschrieben (WMA 2013a). Zusätzlich haben Herausgeber elf wichtiger Journals in Indien angegeben, dass sie nur registrierte Studien zur Veröffentlichung in Betracht ziehen würden (CTRI).

Zum generellen Verständnis über den Ablauf von medizinischen Studien in Indien muss die Rolle der Clinical Research Organizations (CRO) kurz erwähnt werden. Die CROs werden von Pharmafirmen beauftragt, Studien durchzuführen. Seit 2000 gab es einen enormen Zuwachs an CROs in Indien. CROs entwickeln zudem die nötige Infrastruktur, um Studien durchführen zu können. Sie suchen geeignete Kliniken und erstellen Datenbanken von potenziellen ProbandInnen (Srinivasan 2009). Einige AutorInnen betonen die problematische Arbeitsweise der CROs, da diese nur an finanziellem 
Profit interessiert seien (George und Srinivasan 2006).

Insgesamt lässt sich festhalten, dass Indien zum einen als Zielort transnationaler Forschung von der indischen Regierung gefördert wird und hiermit an der Globalisierung im Bereich Biomedizin partizipieren möchte. Dies zeigt sich beispielsweise durch die Gesetzesänderung im Schedule Y der Drugs and Cosmetics Rules im Jahre 2005. Zum anderen ergaben sich in den letzten Jahren auch Veränderungen, die auf bisherige Beispiele unethischer Studien (Sengupta 2009; Jesani 2013) in Indien reagieren und Kontrollen diesbezüglich verschärfen. Hierzu zählt zum Beispiel die Verpflichtung, Studien im CTRI registrieren zu müssen und vor allem die Änderungen des Schedule Y im Jahre 2013, die die Entschädigung von Verletzungen und Todesfällen und die Registrierung von Ethikkomitees betreffen. Hierdurch zeigt sich die Relevanz und Notwendigkeit, sich mit ethischen Aspekten bezüglich transnationaler Forschung in Indien detaillierter zu beschäftigen.

\subsubsection{Gesellschaftliche Rahmenbedingungen}

Indien ist ein komplexes Land, dessen gesellschaftliche Rahmenbedingungen nur schwerlich im Detail $\mathrm{zu}$ fassen sind. Allerdings ist es mit Blick auf die medizinethische Fragestellung dieser Arbeit notwendig, zumindest einige Eckdaten zur aktuellen Gesellschaft Indiens zu kennen. Hierzu gehören einige generelle politische und wirtschaftliche Entwicklungen und Informationen zur Bevölkerung Indiens sowie der Zustand des Gesundheitswesens. Zudem gilt es, insbesondere die gesellschaftliche Stellung von Frauen in Indien genauer zu betrachten.

Seit Indien 1947 die Unabhängigkeit von der Kolonialmacht Großbritannien erreicht hat, gilt das Land als die größte weltweite parlamentarische Demokratie mit 28 Bundesstaaten. Die Hauptstadt ist New Delhi mit circa 16,3 Millionen Einwohnern (Ministry of Home Affairs 2011f, S. 2). Die Währung ist die indische Rupie, wobei ein Euro circa 70 Rupies entsprechen (März 2013) (Auswärtiges Amt 2013). Indiens Wirtschaft boomt derzeit (Wessler 2007). Das Wirtschaftswachstum betrug 2012 3,2\% (Deutschland 2012: 0,7\%) (The World Bank 2013f). Neben der Textil-, Chemie-, Stahl- und Softwarebranche stellt auch die Arzneimittelbranche einen wichtigen Teil der indischen Industrie dar (Central Intelligence Agency 2013). Allerdings arbeiteten 2010 51\% der Beschäftigten in der Landwirtschaft (Deutschland: 2\%) (The World Bank 2013e). Dies verdeutlicht die große Kluft innerhalb Indiens zwischen dem urbanen und ruralen Leben und den damit verbundenen gesellschaftlichen Spannungen. Gemäß dem Human Development Index (HDI), der vom Entwicklungsprogramm der Vereinten Nationen (UNDP) veröffentlicht wird und die durchschnittliche Entwicklung eines Landes widerspiegeln soll, steht Indien immer noch weit hinten, an Stelle 136 von 186 (je niedriger die Zahl, desto höher ist ein Land entwickelt). Anhand des HDI werden Länder in 
sehr hoch, hoch, mittel und niedrig entwickelte Länder eingeteilt. Indien zählt hierbei zu den Ländern mit mittlerem Entwicklungsstand, jedoch im unteren Bereich (United Nations Development Programme 2013). ${ }^{5}$ Zum Vergleich: Deutschland steht an 5. Stelle (United Nations Development Programme 2013). In die Berechnungen des HDI wird unter anderem die Lebenserwartung bei der Geburt, Indikatoren für das Bildungsniveau, wie beispielsweise die erwartete Anzahl von Schuljahren für Kinder im Einschulungsalter, und das Pro-Kopf-Einkommen miteinbezogen (United Nations Development Programme).

Die Bevölkerung Indiens zählt circa 1,2 Milliarden Menschen (2011) (Auswärtiges Amt 2013) und weist in den letzten Jahrzehnten ein enormes Wachstum von 17\% auf (2001-2011) (Ministry of Home Affairs 2011b). Hierbei ist es wichtig zu wissen, dass in Indien viele junge und somit (prinzipiell) erwerbsfähige Menschen leben (The World Bank 2013r). Der Großteil der Bevölkerung (2010 circa $70 \%$ ) lebt in ländlichen Gebieten. Aufgrund der hohen Armut fliehen jedoch viele vom Land in die Stadt, wodurch die Metropolen Indiens schnell wachsen, zum Beispiel die Megacities Mumbai (18,4 Millionen), New Delhi (16,3 Millionen) und Bangalore (8,5 Millionen) (Ministry of Home Affairs 2011d). Indiens Bevölkerung vereint zahlreiche unterschiedliche Ethnien. Es gibt 698 indigene Volksgruppen in Indien, die sich selbst als Adivasi bezeichnen und von der Regierung Scheduled Tribes genannt werden. Diese bilden insgesamt 8,2\% der Bevölkerung. Die indigenen Völker wurden viele Jahre lang diskriminiert und gehören in der Kastengesellschaft zur Gruppe der Kastenlosen beziehungsweise Unberübrbaren, die auch als Dalit (Unterdrückte) bezeichnet werden. Indien vereint auch viele unterschiedliche Religionen. Etwa 80\% der Bevölkerung gehören dem Hinduismus an, 13\% dem Islam, 2\% dem Christentum, 2\% dem Sikhismus und die Restlichen zählen zum Buddhismus, Jainismus, sind Parsen oder sonstiges (Auswärtiges Amt 2013). Zwar hat die indische Verfassung das hinduistische Kastensystem offiziell abgeschafft, es existiert aber aufgrund der noch immer enormen Bedeutung in der Gesellschaft inoffiziell weiter. Das Kastensystem hat eine stark hierarchische Trennung gesellschaftlicher Gruppen zur Folge, das zudem eine Arbeitsteilung mit sich bringt. Es gibt vier Hauptkasten, die hierarchisch von oben nach unten folgendermaßen geordnet sind: Die Brahmanen (Priester), die Kshatriya (Krieger), die Vaishya (Händler) und die Shudra (Bediensteten). Das niedrigste Ansehen haben die Dalits, die außerhalb des Kastensystems stehen und meist sogenannte unreine Arbeiten verrichten. Die Dalits werden von der Regierung als Scheduled Castes bezeichnet und stellen etwa 16\% der Gesamtbevölkerung dar. Die Diskriminierung der Dalits hält auch heute noch an, wobei dies vor allem in ländlichen Gebieten der Fall ist. Um dies abzuschaffen, ist seit 1947 in der Verfassung verankert, dass eine bestimmte Anzahl der Parlamentssitze für die Dalits, entsprechend ihrem Anteil an der Bevölkerung, reserviert werden (Skoda 2007). Sowohl die Diskriminierung indigener Völker als

Einteilung des HDI: sehr hoch: 1-47; hoch: 48-94, mittel: 95-141, niedrig: 142-186, United Nations Development Programme (2013) 
auch der Dalits könnten wichtige Hinweise auf die Entstehung einer möglichen Vulnerabilität in bestimmten Kontexten sein. Ein weiterer Punkt, der die Diversität der indischen Gesellschaft widerspiegelt, ist die Ko-Existenz vieler unterschiedlicher Sprachen (122 im Jahre 2001) (Ministry of Home Affairs 2001). Die offiziellen Landessprachen sind Hindi und Englisch (Auswärtiges Amt 2013). Bemerkenswert ist, dass 2001 nur circa 226.449 InderInnen Englisch als ihre Muttersprache angaben (Ministry of Home Affairs 2001). Die enorme Anzahl unterschiedlicher Sprachen und auch Schriften ist vor allem für die Praxis der informierten Aufklärung (informed consent) von großer Bedeutung. Hier kann die Sorge geäußert werden, dass im Rahmen transnationaler Forschung Einwilligungsformulare auf Englisch und Hindi verwendet werden, die von potenziellen ProbandInnen aufgrund sprachlicher Defizite nicht ausreichend verstanden werden. In diesem Fall könnte aus dem ethisch ungültigen informed consent Vulnerabilität resultieren. Der Schedule $Y$ legt fest, dass Einwilligungsformulare in verständlicher Sprache für die ProbandInnen vorliegen müssen und dass ihre Überprüfung sowie die Korrektheit der Übersetzung zu den Aufgaben der Ethikkomitees zählen (MOHFW 2005, S. 506). Nadig stellte allerdings in einer Studie fest, dass viele Ethikkomitees nicht wissen, dass sie dies überprüfen müssen (Nadig et al. 2011). Ein weiteres Problem hinsichtlich des informed consent und der daraus resultierenden Vulnerabilität stellt die Einschränkung der Freiwilligkeit aufgrund eines unangemessenen Anreizes dar. Diese Sorge ist besonders groß bei potenziellen ProbandInnen, die in Armut leben. In Indien stellt Armut trotz des Wirtschaftsaufschwungs immer noch ein enormes Problem dar. 2010 lebten circa 33\% der Bevölkerung in extremer Armut. Dieser Anteil der Bevölkerung muss mit weniger als 1,25 US-Dollar pro Tag auskommen (The World Bank 2013s). Die Mehrheit der Menschen in Indien, die in großer Armut leben, sind Frauen (Nandal 2005). Zu den Gründen stellt Nandal folgendes fest:

„My attempt to know the extent of poverty did reveal that lack of economic opportunities among women, educational deprivation, gender inequality in access to assets and decision-making produce cycles of poverty for Indian women“" (Nandal 2005, S. 289).

Armut und hohe Verschuldung fordern vor allem in ländlichen Gebieten zahlreiche Opfer und mit der Hoffnung auf ein besseres Leben fliehen viele in die Städte, wobei die meisten in den Slums enden (Petersen 2007).

Bezüglich des informed consent im Kontext transnationaler Biomedizin und als möglicher Faktor, der zu Vulnerabilität führen könnte, ist es zudem wichtig, das Bildungssystem in Indien genauer zu erläutern. Hierbei gilt es insbesondere mögliche Unterschiede bezüglich Bildung und Zugang zu Bildung zwischen Mädchen und Jungen darzulegen. In Indien hat jedes Kind zwischen sechs und 14 Jahren laut Gesetz das Recht auf Zugang zu Bildung, wobei acht Jahre Elementarbildung verpflichtend sind. Darauf folgen zwei Jahre Sekundarstufe. Daran schließen sich zwei Jahre obere Sekundarstufe an, die nach Abschluss dazu berechtigen, an den Aufnahmeprüfungen für die Hochschulen teilzunehmen 
(Hillger 2007). Trotz des per Gesetz vorgeschriebenen Rechts auf Bildung arbeiteten 2006 circa 15\% der Kinder zwischen sieben und 14 Jahren (The World Bank 2013b). Die absolute Zahl der Mädchen im Schulalter, die nicht zur Schule gehen, lag 2010 bei circa 868.000 (The World Bank 2013c), die der Jungen bei circa 806.000 (The World Bank 2013d). Dies steht in erster Linie im Zusammenhang mit der Anzahl von Kindern, die die Schule abbrechen oder nach der Elementarbildung nicht in die Sekundarstufe eintreten, denn die Einschulungsraten sind verhältnismäßig hoch (The World Bank 2013g, 2013h). Der Anteil der Kinder, die nach Abschluss der Elementarbildung in die Sekundarstufe aufstiegen, lag 2010 allerdings bei nur 63\%, wobei hierbei die Anzahl der Kinder, die die Sekundarstufe nicht abschließen, noch nicht mitgezählt wurde (The World Bank 2013y). Betrachtet man die Geschlechterverteilung bezüglich der Einschreibung in die Sekundarstufe oder in die Hochschulen, zeigt sich zwar in der Entwicklung der letzten Jahre eine Verbesserung, es bleibt jedoch immer noch unausgeglichen zu Gunsten der Jungen und Männer. 2010 kamen auf 100 Schüler, die die Sekundarstufe erstmals besuchten, 92 Schülerinnen (The World Bank 2013w). Bezüglich des Beginns eines Hochschulstudiums waren es nur 73 Studentinnen auf 100 Studenten (The World Bank 2013x). Allerdings ist der Anteil der SchülerInnen, die die Sekundarstufe abschließen und innerhalb von fünf Jahren einen Hochschulstudium beginnen, mit nur 18\% im Jahre 2010, insgesamt sehr gering (The World Bank 2013z). Dies lässt sich durch eine Form von Eliteförderung erklären, unter anderem bedingt durch Aufnahmeprüfungen der Hochschulen, wodurch der Zugang zu einem höheren Bildungsabschluss nur bestimmten Bevölkerungsschichten und auch vor allem männlichen Studenten vorbehalten bleibt (Hillger 2007). Der Anteil der Erwachsenen (über 15 Jahre), die lesen und schreiben können, betrug 2006 insgesamt 63\% (The World Bank 2013o). Im Jahre 2011 stieg diese Zahl auf 74\%. Hierbei lag die Alphabetisierungsrate der Männer in Indien bei circa 82\%, die der Frauen bei 65\% (Ministry of Home Affairs 2011c). Zusammenfassend lässt sich festhalten, dass zwar die Einschulungsraten hoch sind und hierbei die Geschlechterverteilung ausgeglichen ist, die Unterschiede der Alphabetisierungsraten hinsichtlich des Geschlechts, der Region und städtischer versus ländlicher Bevölkerung jedoch sehr groß blieben (Ministry of Home Affairs 2011c, 2011a). ${ }^{6}$

Im folgenden Abschnitt sollen Faktoren, die die Gesundheit der indischen Bevölkerung betreffen und hierdurch ethische Aspekte im Rahmen medizinischer Studien beeinflussen könnten, genauer beleuchtet werden. Die Staatsausgaben Indiens für Gesundheit, gemessen am Bruttoinlandsprodukt, lagen 2011 bei 3,9\% (Deutschland: 11,1\%) (The World Bank 2013i). Die Lebenserwartung in Indien beträgt etwa 66 Jahre (2011) (Deutschland: circa 81 Jahre) (The World Bank 2013n). Sie stieg in den letzten Jahren an, wobei Frauen mit 68 Jahren eine höhere Lebenserwartung aufweisen als Männer mit 64 Jahren (2011) (The World Bank 2013m). Anhand von Statistiken über Todesursachen in Indien von

\footnotetext{
${ }^{6}$ Alphabetisierungsraten (2011): In Bihar (Nordosten): circa 64\%; in Kerala (Süden): circa 94\%; in ländlichen Gebieten: circa $68 \%$; in den Städten circa $84 \%$
} 
1970 bis '80 zeigt sich, dass vor allem sogenannte vermeidbare Erkrankungen Haupttodesursachen darstellen, wie beispielsweise Infektionskrankheiten und Ernährungsprobleme (Qadeer 2011, S. 31). Außerdem gibt es große Unterschiede bezüglich der Gesundheitsversorgung in ländlichen Gebieten im Vergleich zu den Städten. Über 70\% der Landbevölkerung wird von nur 20\% der in Indien arbeitenden ÄrztInnen versorgt (Qadeer 2011, S. 68). Ein weiterer wichtiger Indikator für die Gesundheit ist die Anzahl der Kinder pro 1.000 Lebendgeburten, die noch vor Erreichen des fünften Lebensjahres sterben. Diese betrug 2012 in Indien 56 (Deutschland: 4) (The World Bank 2013q). Außerdem waren 2011 nur 72\% der Kinder im Alter zwischen 12 und 23 Monaten gegen Diphtherie, Keuchhusten und Tetanus geimpft (Deutschland: 99\%) (The World Bank 2013j). Borooah veröffentlichte 2004 eine Studie, die zeigte, dass Mädchen hinsichtlich eines kompletten Impfstatus und auch bezüglich einer ausgewogenen und ausreichenden Ernährung benachteiligt sind im Vergleich zu ihren Brüdern oder Jungen im gleichen Alter. Allerdings gab es die Ernährung betreffend keine Diskriminierung der Mädchen, wenn die Mütter lesen und schreiben konnten (Borooah 2004). Die Prävalenz von Unterernährung, also der Anteil der Bevölkerung, der nicht ausreichend Nahrung zu sich nimmt, um den täglichen Energiebedarf regelmäßig abzudecken, betrug 2011 in Indien erschreckende 18\% (The World Bank 2013u). Hinsichtlich des Ernährungszustandes gibt es anscheinend auch teilweise große Unterschiede zwischen Männern und Frauen in Indien (Chorghade et al. 2006). Zusätzlich zur Unterernährung spielt der Zugang zu sauberem Trinkwasser eine entscheidende Rolle für die Gesundheit der Menschen. Diesbezüglich ist es bemerkenswert, dass 2011 nur 35\% der Bevölkerung Zugang zu sanitären Anlagen hatten (The World Bank 2013k). Ein weiterer wichtiger Aspekt hinsichtlich der Gesundheit von Frauen in Indien ist der Anteil der Schwangeren, der pränatal mindestens einmal bezüglich der Schwangerschaft medizinisch betreut wird. Dieser Anteil lag 2008 bei 75\%, somit wurde ein Viertel der Frauen während der Schwangerschaft kein einziges Mal untersucht (The World Bank 2013t). Zudem wurden 2008 nur 52\% der Geburten von medizinischem Fachpersonal betreut (The World Bank 2013a). Hiermit könnte unter anderem die verheerend hohe Müttersterblichkeit in Indien erklärt werden, welche 2010 bei 200 pro 100.000 Lebendgeburten lag (Deutschland: 7 pro 100.000) (The World Bank 2013p). Die Müttersterblichkeit in Indien nahm jedoch in den letzten Jahren stetig ab, 1995 lag diese beispielsweise noch bei 480 pro 100.000 Lebendgeburten (The World Bank 2013p).

Anhand dieser wenigen Faktoren sollte bereits deutlich geworden sein, dass es durchaus nicht völlig absurd ist, Frauen in diesen gesellschaftlichen Bedingungen als zumindest potentiell vulnerabler als Männer einzustufen. Hinzu kommen die sozialen Faktoren, die ihre Stellung im privaten wie öffentlichen Kontext näher bestimmen. Ähnlich wie in anderen Demokratien gab es in den letzten Dekaden Frauen, die vereinzelt zentrale politische Ämter besetzen. So war von 2007 bis 2012 das amtierende Staatsoberhaupt in Indien erstmals eine Frau, Pratibha Patil (Mentschel 2007). Zudem gilt 
Indira Ghandi als entscheidendes Beispiel für Frauen in Indien in einer Machtposition. Als erste und bisher einzige Premierministerin Indiens regierte sie von 1966 bis 1977 und von 1980 bis zur ihrer Ermordung durch ihre Leibwächter 1984 (Kessler 2007). 2012 waren allerdings nur 11\% der Parlamentssitze von Frauen besetzt, was eine deutliche politische Unterrepräsentation darstellt (The World Bank 2013v). Ein sehr wichtiges Beispiel, das eine anhaltende Diskriminierung aufgrund des Geschlechts, sogar schon vor der Geburt, in Indien widerspiegelt, stellt die selektive Abtreibung von weiblichen Föten dar. Dies zeigt sich anhand des unausgeglichenen Geschlechterverhältnisses (Anzahl der Frauen pro 1000 Männer). Beim Zensus 2011 in Indien lag dieses bei 940. Im zeitlichen Verlauf betrachtet, sank das Geschlechterverhältnis seit 1901 zunächst stetig, mit einem Tiefpunkt im Jahre 1991 bei 927, seitdem ist ein Anstieg zu bemerken (Ministry of Home Affairs 2011e, S. 80). Allerdings liegt das Geschlechterverhältnis der Null- bis Sechsjährigen nur bei 914 und ist somit niedriger als der nationale Durchschnitt (Ministry of Home Affairs 2011e, S. 88). Zudem zeigt sich in dieser Altersgruppe eine sinkende Tendenz im Vergleich zum Jahre 2001. Somit scheint das Geschlechterverhältnis zukünftig wieder zu sinken. Des Weiteren zeigten sich 2011 je nach Region große Unterschiede hinsichtlich des Geschlechterverhältnisses (Ministry of Home Affairs 2011e, S. 83). Bedingt durch kulturelle, sozioökonomische und religiöse Faktoren bevorzugen viele Familien in Indien die Geburt eines Sohnes. Ein Grund hierfür ist die vermeintliche finanzielle und emotionale Sicherheit, die ein Sohn den Eltern im Alter bieten kann. Außerdem trägt ein Sohn den Namen der Familie weiter und könnte nach dem Ableben der Eltern ein Familienunternehmen weiterführen. Zusätzlich ist es üblich, dass der Sohn der Familie sich um die Beerdigung und die Bestattungsriten kümmert (Kusum 2000, S. 51). Aufgrund des Brauchs der sogenannten Mitgift bedeutet eine Tochter hingegen meist eine schwere finanzielle Bürde für die Eltern. Außerdem müssen die Eltern ständig befürchten, dass ihre Tochter wegen der eventuell noch nicht vollständig gezahlten Mitgift von ihrem Ehemann oder dessen Familie belästigt oder misshandelt wird. Zusätzlich wird es als schwere Last angesehen, die Jungfräulichkeit der Töchter bis zur Verheiratung erhalten zu können, denn diese ist von großer Bedeutung in der Gesellschaft (Kusum 2000, S. 51). Diese Umstände haben dazu geführt, dass manche Paare das Geschlecht des Fötus ermitteln lassen und im Falle einer Tochter einen Schwangerschaftsabbruch vornehmen lassen (Kusum 2000, S. 52). Allerdings wurde in Indien bereits 1971 mit dem Medical Termination of Pregnancy Act Abtreibung aufgrund des Geschlechts des Fötus verboten. Im Jahre 1994 wurde zusätzlich der Prenatal Diagnostic Techniques (Regulation and Prevention of Misuse Act) verabschiedet, der die Auswahl des Geschlechts aus nicht-medizinischen Gründen verbietet und bei Verstoß unter anderem eine maximale Gefängnisstrafe von drei Jahren festlegt (Kusum 2000, S. 50). Kusum benennt einige Vor- und Nachteile hinsichtlich des Verbots von Tests zur Feststellung des Geschlechts. Ein Nachteil könnte sein, dass hierdurch die Entscheidungsfreiheit von Frauen eingeschränkt und die Anzahl ungewollter Töchter steigen würde. Hinzu käme die Notwendigkeit 
wiederholter Schwangerschaften bis ein Sohn geboren würde und damit verbundene gesundheitliche Risiken. Zuletzt könnte ein Verbot die heimliche Durchführung von Tests und Schwangerschaftsabbrüchen verstärken, womit medizinische Risiken und eine mögliche Ausbeutung verbunden seien. Vorteile eines Verbots seien, dass ein unausgewogenes Geschlechterverhältnis wieder ausgeglichen werden könnte und somit die Gefahr von Polyandrie verhindert würde. Zudem könnten häufige Tests und Schwangerschaftsabbrüche die Gesundheit der Frauen gefährden (Kusum 2000, S. 55).

Wie bereits erwähnt, steht vor allem der Brauch der Mitgift in Indien im Zusammenhang mit Diskriminierung von Mädchen und Frauen. Laut Tradition ist es üblich, dass die Eltern einer Tochter bei deren Heirat eine Mitgift in Form von Vermögen für die Zukunft und das Zuhause des neuen Paares an den Ehemann und dessen Familie zahlen (Srinivasan 2005, S. 595). Hierzu muss erwähnt werden, dass der Brauch der Mitgift seit 1961 laut des Dowry Probibition Act per Gesetz verboten wurde. Dieses Gesetz wurde 1984 und erneut 1986 verändert, wobei unter anderem die Vorschrift zur Einsetzung von sogenannten dowry probibition officers zur Überprüfung der Einhaltung des Gesetzes festgelegt wurde (Singh 1995, S. 392). Allerdings scheint diese Vorschrift in den meisten Bundesstaaten keine Umsetzung zu finden (Singh 1995, S. 392). Die Tradition der Mitgift zieht sich trotz des Gesetzes durch alle Gesellschaftsschichten und Kasten. Hochzeiten ohne Mitgift werden seltener (Srinivasan 2005, S. 608). Srinivasan erläutert hierzu: „Dowry has become an all caste/class phenomenon and average dowry payments have risen“ (Srinivasan 2007, S. 857). Srinivasan beschreibt Auswirkungen der Mitgift auf den Status der Frau innerhalb ihrer Ehe. Zum einen verbessere sich durch die Mitgift die finanzielle Lage des ehelichen Hausstandes, wodurch eventuelle ökonomische Belastungen verringert würden. Zum anderen spiegelt die Mitgift den Wert des Ehemanns auf dem Heiratsmarkt wider. Somit könne eine hohe Mitgift den sozialen Stand des Ehemanns aufwerten und hierdurch der Weg für eine unbeschwerte Beziehung geebnet werden (Srinivasan 2007, S. 862). Für die Familie der Braut stellt die Mitgift eine schwere finanzielle Bürde dar. Zudem ist es Eltern aus soziokulturellen Gründen untersagt finanzielle Unterstützung von ihrer verheirateten Tochter anzunehmen. Die möglichen Folgen unerfüllter Mitgiftansprüche von Seiten des Ehemanns oder der Schwiegereltern können für Eltern in vielerlei Hinsicht sehr belastend sein (Srinivasan 2005, S. 595). Srinivasan zitiert ein indisches Sprichwort, das den sozialen Status von Töchtern verdeutlicht: „Bringing up a daughter is like watering a neighbor's plant“" (Srinivasan 2005, S. 595). Für eine Tochter Mitgift zahlen zu müssen, stellt somit die vorrangige Rechtfertigung für den gezielten Schwangerschaftsabbruch weiblicher Föten und Kindestötung von Töchtern dar (Srinivasan 2007, S. 858). Bemerkenswert ist, dass sich laut einer Studie von Srinivasan viele Frauen gegen die Abschaffung der Mitgift aussprechen (Srinivasan 2007, S. 858). Eine hohe Mitgift sei beispielsweise wichtig, um mit einem guten Mann verheiratet zu werden und sie vor Gewalt in der Ehe zu schützen. Durch die Mitgift könne außerdem der Status der Frau innerhalb der Ehe aufgewertet werden. Abgesehen davon bleiben Mitgiftforderungen eine der wichtigsten 
Ursachen für Gewalt, Belästigung und Tod von Frauen in Indien (Srinivasan 2005, S. 595). Wenn Frauen von ihren Ehemännern oder deren Familien umgebracht werden, weil die Mitgift nicht ausreichend sei, spricht man von sogenannten Mitgiftmorden. Zwischen den Jahren 1999 und 2003 zählte das National Crime Records Bureau jährlich etwa 6.000 bis 7.000 mit Mitgift assoziierte gemeldete Todesfälle (Srinivasan 2007, S. 858). Laut einer Studie von Srinivasan geht eine hohe Mitgift für Ehefrauen mit einem niedrigeren Risiko, häusliche Gewalt zu erfahren einher. Srinivasan ergänzt hierzu: „[...] it may not be dowry per se that affords protection but the extent to which a woman owns and controls dowry as 'her' asset that has a bearing on her security“ (Srinivasan 2007, S. 872). Ein hoher Bildungsstand und ein hohes Einkommen der Ehefrauen sind jedoch mit einem erhöhten Risiko für häusliche Gewalt assoziiert. Srinivasan erklärt die hierbei erhöhte Gewaltbereitschaft der Ehemänner durch eine mögliche Gefährdung ihrer Autorität und einer Bedrohung der geschlechtsspezifischen Normen (Srinivasan 2007, S. 872). Insgesamt gab es in den Jahren 1989 bis 1999 einen Anstieg gemeldeter Fälle von Gewalt gegenüber Frauen von fast 102\%, wobei davon ausgegangen werden muss, dass die überwiegende Mehrheit der Fälle nicht gemeldet wird (Sharma und Gupta 2004, S. 116). Bei einer Studie in einem Slum in Bangalore bezüglich häuslicher Gewalt gaben 56\% der Frauen an in ihrer Ehe Gewalt erfahren zu haben. Frauen, deren Familien nach der Hochzeit nach einer zusätzlichen Mitgift gefragt wurden, erfahren mit einer erhöhten Wahrscheinlichkeit häusliche Gewalt (Rocca et al. 2009, S. 577). Es gibt noch weitere Beispiele für Gewaltverbrechen und die Nichtbeachtung der Rechte indischer Frauen, allerdings würde es den Rahmen dieser Arbeit sprengen, diese vollständig zu behandeln. Einen Einblick in die Problematik geben die oben genannten Beispiele bezüglich Geschlechtsselektion, Mitgift und Gewalt. Als Zusammenfassung hinsichtlich der Diskriminierung von Frauen in Indien soll ein Zitat von Sharma und Gupta dienen:

„Religion, customs, age-old prejudices, etc. have put Indian women in a subservient and exploitable position in many domains of life. Low rates of participation in education, lack of economic independence, value biases operating against them, etc, have resulted in the women being dependent on men folk and other institutions of authority like the family, neighborhood and the society. They are usually ignorant of their rights and even if they are not, they do not have easy access to justice" (Sharma und Gupta 2004, S. 114).

Dies trifft aber nicht auf alle Frauen in Indien zu. Ein Beispiel hierfür ist Rita Banerji, eine Autorin und Frauenaktivistin in Indien. Sie gründete 2006 die 50 Million Missing Campaign, die ein internationales öffentliches Bewusstsein für den Genożid an Indiens Frauen schaffen will und Aktionen durchführt, um dies zu verändern (Banerji). In der Verfassung Indiens wird zudem die Gleichheit und Nichtdiskriminierung aufgrund von Geschlecht festgelegt. 1980 unterzeichnete Indien die sogenannte Women's Convention, Convention on the Elimination of All Forms of Discrimination Against Women (Singh 1995, S. 375). Außerdem wurde 1995 das Department of Women and Child Development von der Regierung aufgebaut und im Jahre 2006 wurde dieses zum Ministry of Women and Child Development hochgestuft. 
Seine Aufgabe ist das Ausarbeiten von Plänen, Grundsätzen und Programmen sowie eine Erlassung oder Änderung der Gesetzgebung zur Förderung von Frauen und Kindern. Hierbei wurde unter anderem 2006 der Protection of Women from Domestic Violence Act gesetzlich verfügt. Des Weiteren gibt es die 1981 gegründete All Indian Democratic Women's Association, eine unabhängige Organisation, die daran arbeitet, Demokratie, Gleichheit und Emanzipation für Indiens Frauen zu erreichen. Diese Organisation ist in 22 Bundesstaaten vertreten und zählt über neun Millionen MitgliederInnen (All India Democratic Women's Association 2007). Eine weitere wichtige Frauenorganisation in Indien, die vor allem die gesundheitlichen Bedürfnisse von Frauen in den Vordergrund stellt, ist die Resource Group for Women and Health (Sama). Sama wurde 1999 von Frauen mit feministischem und medizinischem Hintergrund gegründet (Sama Resource Group for Women and Health). Sama versucht ein weiter gefasstes Verständnis von Gesundheit zu realisieren. Menschen sollen darin bestärkt werden, physische, soziale und politische Umstände, die sich auf ihre Gesundheit auswirken, beeinflussen zu können. Hierfür sollen Frauen unter anderem hinsichtlich ihrer Rechte im Gesundheitswesen geschult werden (Sama Resource Group for Women and Health).

Fazit: Trotz vieler erfolgreicher Anstrengungen durch Gesetzgebungen, Organisationen und Kampagnen, die Rechte von Frauen in Indien bezüglich verschiedener Ebenen zu gewährleisten, wurden innerhalb dieses Kapitels auch viele Aspekte aufgezeigt, die eine anhaltende und gravierende Diskriminierung vieler Frauen in Indien verdeutlichen. Diese Ansammlung von Aspekten könnte im Kontext medizinischer Forschung in Indien zu einer möglichen Vulnerabilität von Studienteilnehmerinnen führen. Durch die Diskriminierung und Benachteiligung vieler Frauen in Indien, die zahlreiche Lebensbereiche betreffen, wie beispielsweise Zugang zu Bildung oder Gesundheitsversorgung sowie eine hohe Wahrscheinlichkeit, Gewalt ausgesetzt zu sein, könnte sich eine erhöhte Wahrscheinlichkeit ergeben, Unrecht zu erfahren. Demnach benötigten diese Frauen besonderen Schutz. Inwiefern Frauen in Indien im Kontext transnationaler Biomedizin eine erhöhte Wahrscheinlichkeit aufweisen, Unrecht zu erfahren, soll daher anhand der Analyse der Beispielstudien geprüft werden. Die oben vorgestellten gesellschaftlichen Faktoren sollen dabei miteinbezogen werden. 
5. Analyse und Interpretation von zwei medizinischen Studien in Indien im Hinblick auf eine mögliche Vulnerabilität der Studienteilnehmerinnen

Das folgende Kapitel beinhaltet die Analyse und Interpretation zweier transnationaler medizinischer Fallstudien, die mit Frauen in Indien durchgeführt wurden. Die Auswahl beider Fallstudien wurde in Kapitel 2 beschrieben. Die Fallstudien A und B werden jeweils nacheinander bearbeitet. Es folgt zunächst eine deskriptive Analyse der jeweiligen Studie, die Hintergrundinformationen zu dem erforschten Krankheitsbild, die Zielstellung und Methodik der Studie, Informationen zu den Studienbedingungen und -teilnehmerInnen sowie eine Beschreibung des Studienverlaufs und der Ergebnisse beinhaltet.

Daraufhin werden im Interpretationsteil der jeweiligen Fallstudie Fragen und Zusatzinformationen diskutiert, die ethische Aspekte bei der Planung und Durchführung dieser Studien betreffen. Diesbezüglich gilt es die in Kapitel 3.2 und 3.3 erläuterten ethischen Aspekte hinsichtlich Forschung mit Frauen beziehungsweise Forschung in Entwicklungsländern als Grundlage miteinzubeziehen. Im nächsten Schritt werden sowohl die Informationen der Analyse als auch die Erörterungen ethischer Konflikte mit Blick auf eine mögliche Vulnerabilität der Studienteilnehmerinnen der jeweiligen Fallstudie normativ bewertet. Hierzu werden die in Kapitel 3.4 aufgestellten Vulnerabilitätskriterien angewendet und einzeln geprüft. Im Anschluss an die Bearbeitung beider Fallstudien folgt ein Überblick und ein Vergleich der Ergebnisse beider Fallstudien.

\subsection{Fallstudie A: 'Safety and Immunogenicity Study of tgAAC09, a Gag-PR-RT AAV $\underline{\text { HIV Vaccine }}{ }^{17}$}

Vulnerabilität im Kontext einer HIV-Studie zu untersuchen, ist insofern von besonderer Bedeutung, da HIV-Studien häufig mit vulnerablen Forschungspopulationen durchgeführt werden (Thomas 2004). Außerdem gab es unethische HIV-Studien in der Vergangenheit, die mit hoher Wahrscheinlichkeit mit vulnerablen Gruppen durchgeführt wurden (siehe hierzu Kapitel 1). Zusätzlich bergen HIV-Studien unter anderem sozioökonomische Risiken für die StudienteilnehmerInnen, da die Teilnahme an einer HIV-Studie zu Stigmatisierung und Diskriminierung führen kann (Thomas 2004). Diese besonderen Risiken könnten wiederum zu Vulnerabilität im Rahmen einer Studienteilnahme führen.

Die zu analysierende Studie wurde 2007 mit dem Titel A Phase I Randomized, Placebo-Controlled, DoubleBlind Dose-Escalation Trial to Evaluate the Safety and Immunogenicity of $\operatorname{tg} A$ AC09, a Gag-PR-RT AAV HIV Vaccine bei clinicaltrials.gov registriert (clinicaltrials.gov). Die Studie wurde sowohl in Deutschland und

\footnotetext{
${ }^{7}$ Kurztitel der Studie bei Clinicaltrials.gov (2007)
} 
Belgien als auch in Indien durchgeführt, wobei sich die Analyse auf die Studie in Indien konzentriert. Ziel der Studie war es, die Sicherheit und Immunogenität der HIV-Impfung tgAAC09 an gesunden Erwachsenen zu testen (Mehendale et al. 2008, S. 168). Begründet wird die Initiierung der Studie durch die hohe Krankheitslast von HIV weltweit und durch die limitierenden Faktoren sowohl hinsichtlich der Therapie- als auch der Präventionsmöglichkeiten (Mehendale et al. 2010, S. 169). Somit ist das Fernziel, eine präventive Impfung gegen HIV zu entwickeln (Mehendale et al. 2008, S. 873). Zu dieser Studie wurden zwei Artikel veröffentlicht. Diese sind:

1) Mehendale S, van Lunzen J, Clumeck N, Rockstroh J, Vets E, Johnson PR, Anklesaria P, Barin B, Boaz M, Kochhar S et al. (2008): A phase 1 study to evaluate the safety and immunogenicity of a recombinant HIV type 1 subtype $C$ adeno-associated virus vaccine. AIDS Res Hum Retroviruses 24, 873-880.

2) Mehendale S, Sahay S, Thakar M, Sahasrabuddhe S, Kakade M, Shete A, Shrotri A, Spentzou A, Tarragona T, Stevens G et al. (2010): Safety \& immunogenicity of tgAAC09, a recombinant adeno-associated virus type 2 HIV-1 subtype C vaccine in India. Indian J Med Res $\underline{132}, 168$ 175.

Für die Analyse der Fallstudie wurde hauptsächlich die zuletzt genannte Publikation verwendet, da der Fokus auf dem indischen Studienteil legt. Das Abstract der zweiten Publikation lautet:

„Background \& objective: A phase I trial of adeno-associated based HIV-1 subtype C vaccine (tgAAC09) was conducted at two sites in German and Belgium and one site in India. This paper reports the safety and immunogenicity of tgAAC09 in healthy adult Indian volunteers.

Methods: Between January 2005 and December 2006, 30 consenting volunteers were enrolled in the placebo controlled double-blind dose escalation trial $8\left[3 \times 10^{9}, 3 \times 10^{11}\right.$ DNase resistant particles $(\mathrm{DRPs}) / \mathrm{ml}$. Single injection of the candidate vaccine was administered to ten volunteers randomized in 8:2 ratio in vaccine and placebo arms at each dosage level.

Results: The mean age of study volunteers (16 men and 14 women) was $34 \mathrm{yr}$. Six local reactogenicity events and 14 systemic reactogenicity events like malaise, fever, headache and myalgia were reported, both were dose-dependent. The difference events between the adverse events reported by vaccine and placebo recipients (79 and 67\%) was not significant. A modest IFN- $\gamma$ ELISPOT response [248 spot forming units (SFU/million cells] was detected in one volunteer from high dose group and low response (56 and $75 \mathrm{SFU} /$ million cells) in two volunteers in low and mid-dose groups. A post-vaccination dosedependent increase was observed in anti AAV2 neutralizing titres. None of the volunteers showed appositive antibody response to HIV-1.

Interpretation \& conclusions: The trial was a benchmark in phase I clinical evaluation of HIV candidate vaccines in India. The vaccine was generally well tolerated and raised no safety concerns. The vaccine was found to be weakly immunogenic. It is essential to understand the role of pre-existing immunity against vectors and significance of evaluation in a prime-boost strategy" (Mehendale et al. 2010, S. 168). 


\section{$\underline{5.1 .1 \text { Hintergrundinformationen und aktueller Forschungsstand zu Fallstudie A }}$}

Um die Studie besser zu verstehen, müssen nach der Erläuterung der Zielstellung der Studie zunächst allgemeine medizinische und epidemiologische Informationen zum Humanen Immundefizienz-Virus (HIV) und dem hierdurch verursachten Acquired Immunodeficiency Syndrome (AIDS), also auch der aktuelle Forschungsstand zu Therapie- und Präventionsmöglichkeiten, erläutert werden. Für den Kontext dieser Studienanalyse ist es zudem wichtig, die Situation von HIV/AIDS in Indien genauer zu beleuchten und dies insbesondere auch mit Blick auf Frauen in Indien. Diese Informationen sollen dann als Hintergrund dienen, um die eigentliche Analyse der Fallstudie in den gesellschaftlichen Kontext von Indien einordnen zu können.

Im Jahre 1981 wurde AIDS erstmals als eine neue Erkrankung beschrieben und 1983 festgestellt, dass HIV der ursächliche Erreger ist (Rockstroh 2012). HIV befällt Immunzellen, die das Glykoprotein CD4 auf ihrer Oberfläche tragen, sogenannte CD4-positive Zellen, wie beispielsweise T-Zellen und Makrophagen (Rubbert-Roth und Behrens 2012). Das Virus zerstört oder schränkt die Funktion dieser Zellen ein, wodurch eine Immunschwäche entsteht (UNAIDS 2008). Man unterscheidet zwischen HIV1 und HIV-2, wobei letzteres für weniger als 1\% der weltweiten HIV-Infektionen verantwortlich ist (Rubbert-Roth und Behrens 2012). Falls nicht anders gekennzeichnet, ist in dieser Arbeit stets HIV-1 gemeint. Die wichtigsten Übertragungswege von HIV sind folgende: Ungeschützter sexueller Kontakt mit einem infizierten Partner, gemeinsame Verwendung von Spritzutensilien, vor allem bei Drogenabhängigen oder von einer HIV-positiven Mutter auf ihr Neugeborenes vor oder während der Geburt oder durch Stillen (Rockstroh 2012). Der Verlauf der HIV-Infektion beginnt nach der Erstinfektion mit der akuten Phase, die bei manchen PatientInnen unter anderem mit Fieber und Lymphknotenschwellungen einhergeht. Darauf folgt die chronische Phase, die asymptomatisch verläuft und unbehandelt acht bis zehn Jahre dauern kann. Daran an schließt sich AIDS, das anhand bestimmter Erkrankungen, die mit einer Immunschwäche einhergehen, und anhand der Anzahl der CD4-positiven Zellen definiert wird (Rockstroh 2012). Die AIDS-definierenden Erkrankungen führen unbehandelt nach individuell unterschiedlich langer Zeit zum Tod der PatientInnen (Rockstroh 2012).

Seit 1987 gibt es zur Behandlung von HIV Medikamente, die als antiretrovirale Therapie (ART) bezeichnet werden und seither ständig weiterentwickelt wurden. Inzwischen ist es möglich, mithilfe von ART und weiterer Substanzklassen die Virusvermehrung dauerhaft zu unterdrücken und das Fortschreiten der Erkrankung zu verhindern. Allerdings stellen die zwingend regelmäßige Einnahme und die Verträglichkeit der Medikamente limitierende Faktoren für die Behandlung dar. Des Weiteren sind mögliche Langzeittoxizitäten der Medikamente noch unklar (Rockstroh 2012). Die Kosten für ART sind relativ hoch und belaufen sich bei üblichen Dreifachkombinationstherapien auf circa 14.000 bis 21.000 Euro pro PatientIn pro Jahr (Hoffmann 2012). Zudem entwickeln 30\% der PatientInnen trotz regelmäßiger Einnahme und Aufrechterhaltung der Unterdrückung der Viruslast resistente Viren. 
Dies kann zum Versagen der gängigen Therapiemöglichkeiten und zur Übertragung und Neuinfektion von Viren führen, die mit den aktuell verfügbaren Medikamenten eventuell nicht beherrschbar sind (Demberg und Robert-Guroff 2012). Hierdurch ergibt sich die dringende Notwendigkeit für die Entwicklung neuer Therapiestrategien und Präventivmaßnahmen (Demberg und Robert-Guroff 2012).

Die effektivsten Präventivmaßnahmen zur Verhinderung einer HIV-Infektion sind die Verwendung von Kondomen, sexuelle Abstinenz oder die ausschließliche Einmalverwendung von Spritzbesteck bei Drogenabhängigen (Demberg und Robert-Guroff 2012). Des Weiteren sind Mikrobizide, die als Gel vaginal angewendet werden, eine in der Forschung befindliche Methode zur Reduzierung von HIVInfektionen. Die meisten der in Studien getesteten Mikrobizide zeigten jedoch nur eine geringe Wirkung und manche förderten möglicherweise gar eine Infektion mit HIV (Demberg und RobertGuroff 2012). Es gibt noch weitere Methoden beziehungsweise Medikamente, die als Maßnahme zur Vorbeugung von HIV-Infektionen erforscht werden (Demberg und Robert-Guroff 2012). Es würde jedoch den Rahmen dieser Arbeit übersteigen, diese zusätzlich zu erläutern.

Mit Blick auf die zu analysierende Fallstudie ist es allerdings wichtig, den aktuellen Forschungsstand von HIV-Impfungen genauer aufzuzeigen. Es werden sowohl therapeutische als auch präventive HIVImpfungen erforscht (Demberg und Robert-Guroff 2012). Die therapeutischen Impfungen zeigten in Studien zwar erwünschte Wirkungen, allerdings sind die aktuellen Therapiemöglichkeiten den therapeutischen Impfungen bisher noch überlegen (Demberg und Robert-Guroff 2012). Die großen Bemühungen eine präventive Impfung zu entwickeln, die als einziges Mittel gilt, um die HIV-Pandemie unter Kontrolle zu bringen (Harrer 2012) und die vor allem auch für Entwicklungsländer wichtig wäre, da die notwendige Therapie häufig nicht verfügbar ist, sind bisher gescheitert (Demberg und RobertGuroff 2012). Der bisher größte Erfolg zeigte sich bei einer Placebo-kontrollierten Studie, die in Thailand mit 16.000 ProbandInnen durchgeführt wurde und eine Reduktion der HIV-Neuinfektionen von 31\% ergab (Harrer 2012). In der Vergangenheit wurden verschiedene Möglichkeiten für eine HIVImpfung erforscht, die jedoch bisher erfolglos blieben (Harrer 2012). Ein neuer Ansatz ist eine sogenannte genetische Immunisierung. Hierbei handelt es sich um eine passive Immunisierung (Harrer 2012). Genetische Immunisierung bedeutet, dass Gene von neutralisierenden Antikörpern oder Gene von effektiven T-Zell-Rezeptoren mithilfe eines Vektors in immunkompetente Zellen transferiert werden. Durch den Gentransfer werden diese Zellen dann angeregt, Antikörper zu bilden beziehungsweise T-Zell-Rezeptoren zu exprimieren. (Harrer 2012). Diesbezüglich zeigten sich in Tierversuchen vielversprechende Erfolge (Demberg und Robert-Guroff 2012). Als Überträger der Gene, also Vektoren, werden unter anderem Adenovirus-Vektoren, Canary-Pox-Viren und Adenovirusassoziierte Viren verwendet (Harrer 2012). Zu den hauptsächlichen Schwierigkeiten bei der Entwicklung eines HIV-Impfstoffes zählt unter anderem die hohe genetische Vielfalt des Virus und dass die vom Immunsystem entwickelten Antikörper meist spezifisch für den jeweiligen Virustyp der PatientInnen sind. Außerdem entkommt das Virus häufig durch Fluchtmutationen einer 
Immunantwort. Darüber hinaus besteht in der Pharmaindustrie nur ein geringes Interesse einen Impfstoff zu entwickeln (Barouch 2008).

Zudem ist es an dieser Stelle notwendig, einige wesentliche epidemiologische Daten zu HIV und AIDS weltweit zu nennen. Laut des 2013 veröffentlichten UNAIDS Report on the global AIDS epidemic sank die weltweite Neuinfektionsrate von HIV seit 2001 bis 2012 um 33\%, weist jedoch regional große Unterschiede auf (UNAIDS 2013, S. 4ff). Zudem ist die Anzahl der Neuinfektionen insgesamt betrachtet immer noch sehr hoch. Im Jahre 2012 betrug sie geschätzte 2,3 Millionen (UNAIDS 2013, S. 4). Insbesondere aufgrund des besseren Zugangs zu ART, vor allem auch in einkommensschwachen Ländern und der sinkenden Neuinfektionsrate, sank weltweit die jährliche Anzahl der AIDS assoziierten Todesfälle von geschätzten 2,3 Millionen im Jahre 2005 auf 1,6 Millionen im Jahre 2012 (UNAIDS 2013, S. 4). Die Anzahl der Personen weltweit, die mit HIV leben, stieg allerdings von 29,4 Millionen 2001 auf 35,3 Millionen im Jahre 2012 (UNAIDS 2013, S. 4). Dies erklärt UNAIDS durch die Reduzierung der AIDS assoziierten Todesfälle und der lebensverlängernden Wirkung von ART (UNAIDS 2010, S. 23). In den letzten Jahren stieg zwar die Anzahl der Personen, die Zugang zu ART haben, auch in Ländern mit niedrigem und mittlerem Einkommen. So hatten in diesen Ländern im Jahre 2012 9,7 Millionen Menschen mit HIV-Infektion Zugang zu ART (UNAIDS 2013, S. 6). Allerdings haben trotz der Verbesserungen nur 34\% von 28,4 Millionen Menschen in Ländern mit niedrigem und mittlerem Einkommen, die Anspruch auf ART hätten, tatsächlich Zugang dazu (UNAIDS 2013a, S. 6).

Nach der Darstellung allgemeiner Informationen ist es nun wichtig, die Situation von Frauen hinsichtlich HIV und AIDS genauer zu beschreiben. Weltweit betrachtet sind etwas über die Hälfte $(52 \%)$ aller HIV-Infizierten Frauen (UNAIDS 2010, S. 23). In der Sub-Sahara, dem Zentrum der weltweiten AIDS Epidemie, liegt der Anteil der Frauen unter allen HIV-Infizierten bei 57\% (UNAIDS 2013, S. 78). Gerade junge Frauen weisen im Vergleich zu Männern in derselben Altersgruppe ein sehr hohes Risiko für eine HIV-Infektion auf (UNAIDS 2013, S. 78). Bei ungeschütztem sexuellen Kontakt sind Frauen im Vergleich zu Männern einem bis zu achtmal höherem Risiko ausgesetzt, sich mit HIV zu infizieren. Da die Schleimhautoberfläche des weiblichen Genitaltraktes größer und empfindlicher ist als die des männlichen Genitaltraktes, entstehen leichter Verletzungen der Schleimhaut und hierdurch besteht eine höhere Gefahr der HIV-Infektion (Haberl 2012). Weitere Gründe für eine erhöhte Anfälligkeit von Frauen, sich mit HIV zu infizieren, sind folgende:

„Gender disparities in terms of access to education, resources, income, political power, coupled with incidences of sexual violence, coercion, social dislocation in conflict situations like war etc. or owing to migration for work, serve to increase the risk of HIV infection to women through unprotected sexual intercourse" (National AIDS Control Organisation 2008, S. 6).

Insbesondere sexualisierte Gewalt gegenüber Frauen erhöht deren Risiko einer HIV-Infektion. Studien in Uganda und Südafrika zeigten, dass Frauen, die sexualisierte Gewalt in ihrer Partnerschaft erlebten, 
im Vergleich zu Frauen, die keine Gewalt erfahren hatten, eine doppelt so hohe Wahrscheinlichkeit aufwiesen, sich mit HIV zu infizieren (UNAIDS 2013, S. 80).

Schätzungsweise 80\% der Neuinfektionen mit HIV treten in Entwicklungsländern auf, wobei hauptsächlich Länder in Sub-Sahara Afrika und Südostasien stark betroffen sind (Mehendale et al. 2010, S. 169). Im Allgemeinen sind HIV und AIDS mit Armut, einem niedrigen Bildungsstatus und Bevölkerungen, die Minderheiten angehören, assoziiert (Demberg und Robert-Guroff 2012). Die dringend notwendige flächendeckende Bereitstellung von ART weltweit bleibt trotz des verbesserten Zugangs in den letzten Jahren aufgrund verschiedener Aspekte erschwert. Zum einen stellen die hohen Kosten von ART einen limitierenden Faktor dar. Zum anderen sind der Beginn und die Erhaltung einer erfolgreichen Behandlung von politischen und soziologischen Bedingungen abhängig, wie beispielsweise der Stabilität der Regierung, dem Gesundheitssystem, Transportmöglichkeiten und dem Zugang zu ausreichender Nahrung (Demberg und Robert-Guroff 2012). Zusätzlich werden HIV oder AIDS in Entwicklungsländern häufig erst spät diagnostiziert, wodurch aufgrund eines weit fortgeschrittenen Krankheitsstatus eine erfolgreiche Therapie unwahrscheinlich wird (Demberg und Robert-Guroff 2012). Des Weiteren führen soziale Gegebenheiten dazu, dass viele Personen keine Therapie erhalten: „Social barriers such as personal and family shame or blame and societal stigma often paired with insufficient education on HIV/AIDS, can also prevent individuals from seeking treatment" (Demberg and Robert-Guroff 2012, S. 2).

Für diese Arbeit ist es nun von besonderer Bedeutung, den Blick auf die Situation von HIV und AIDS in Indien zu richten und hierbei in einem nächsten Schritt insbesondere Frauen in Indien in den Fokus zu rücken. Im Jahre 2012 lebten in Indien schätzungsweise 2,1 Millionen Menschen mit HIV (UNAIDS 2012a). Indien ist damit nach Südafrika und Nigeria das Land mit der drittgrößten Krankheitslast von HIV (UNGASS: United Nations General Assembly Special Session 2010, S. 1). Die Prävalenz von HIV bei Erwachsenen betrug 2012 in Indien circa 0,3\% (Deutschland: 0,1\%) (UNAIDS 2012a). Um die HIV-Epidemie in Indien aufzuhalten, wurde 1992 erstmals ein National AIDS Control Programme (NACP) eingeführt und die National AIDS Control Organisation (NACO), zugehörig zum Ministry of Health and Familiy Welfare, gegründet (NACO 2012, S. IX). Das dritte NACP, durchgeführt von 2007 bis 2012, beinhaltet unter anderem folgende Ziele: Verhinderung von Neuinfektionen in Hochrisikogruppen und der Allgemeinbevölkerung mithilfe gezielter Interventionen und Bereitstellung von Pflege, Unterstützung und Therapie für Menschen, die mit HIV leben (NACO 2012, S. IX). Hierdurch zeigt die HIV-Epidemie in Indien in den letzten Jahren eine verstärkt rückläufige Tendenz. Die Neuinfektionsrate von Erwachsenen zwischen 15 und 49 Jahren sank beispielsweise von 2001 bis 2012 um mehr als 50\% (UNAIDS 2013, S. 12). Der Anteil der Personen in Indien, die ART benötigen und tatsächlich Zugang dazu haben, lag 2011 allerdings nur bei circa 51\% (UNAIDS 2013, S. A84). 
Insgesamt benötigen circa 1 Million Menschen in Indien ART, was bedeutet, dass schätzungsweise fast eine halbe Million Menschen mit HIV-Infektion in Indien keinen Zugang zu ART haben (UNAIDS 2013, S. A84). Die Krankheitslast von HIV in Indien zeigt regional große Unterschiede auf. Auf nur sechs Bundesstaaten verteilen sich zwei Drittel der gesamten Krankheitslast (UNAIDS 2009). Der hauptsächliche Übertragungsweg von HIV in Indien ist heterosexueller Geschlechtsverkehr (NACO 2012, S. 1). Die hauptsächliche Krankheitslast von HIV und AIDS in Indien betrifft sogenannte Hochrisikogruppen. Hierzu zählen Sexarbeiterinnen, Männer, die Sex mit Männern haben (MSM) und Drogenabhängige (Intravenous Drug User. IDU) (UNGASS 2010, S. 11ff). In den Hochrisikogruppen zeigt sich eine 10 bis 20 mal höhere HIV-Prävalenz verglichen zur restlichen Bevölkerung (UNAIDS 2009). Das Voranschreiten der HIV-Epidemie in Indien wird hauptsächlich von Männern verursacht, die ungeschützten Geschlechtsverkehr mit Sexarbeiterinnen haben (UNGASS 2010, S. 11). Die HIVEpidemie in Indien breitet sich sowohl von Hochrisikogruppen zu Niedrigrisikogruppen als auch von städtische in ländliche Gebiete aus (Excler 2005).

Insbesondere für den Kontext dieser Arbeit gilt es nun die Situation von Frauen in Indien hinsichtlich HIV und AIDS aufzuzeigen. In Indien leben circa 930.000 Frauen mit HIV und stellen 39\% der Gesamtheit der HIV-Infizierten in Indien dar (NACO 2012, S. 5). Demnach leben mehr Männer in Indien mit HIV als Frauen. Über 90\% der Frauen in Indien infizierten sich durch ihren Ehemann oder Partner (UNGASS 2010, S. 3). Hierbei ist es vor allem das risikoreiche Verhalten der Ehemänner und Partner und meist nicht das eigene Verhalten, das Frauen anfällig für eine HIV-Infektion macht (UNGASS 2010, S. 3). Aus vorherrschenden Auffassungen von Männlichkeit und Weiblichkeit resultiert häufig, dass Frauen in Indien wenig Kontrolle und Macht über ihre sexuellen Beziehungen haben, auch innerhalb der Ehe. Aufgrund sozialer Normen, die beinhalten, dass Frauen kein sexuelles Wissen haben sollten, stellen sowohl Abstinenz als auch die Verwendung von Kondomen üblicherweise keine Option zur HIV-Prävention für diese dar (NACO 2007). Zudem ist vielen Frauen in Indien der Zugang zu Bildung verwehrt (siehe hierzu Kapitel 4.2.2). Dies bedeutet, dass häufig notwendiges Wissen und Informationen fehlen, die zu einer Änderung des Verhaltens führen, um HIVNeuinfektionen zu verhindern. Darüber hinaus ist Gewalt gegenüber Frauen stark mit der Gefahr einer HIV-Infektion verknüpft. Frauen in Indien sind durch Vergewaltigungen, Inzest, Übergriffe durch Familienmitglieder oder Freunde, sexualisierter Gewalt am Arbeitsplatz oder im Rahmen von Menschenhandel verstärkt einer HIV-Infektion ausgesetzt (NACO 2007). Zusätzlich haben viele Frauen in Indien einen eingeschränkten Zugang zu Gesundheitsversorgung, da ihrer Gesundheit eine geringe Priorität beigemessen wird und dies ist bezüglich der Prävention und Therapie von HIV von großem Nachteil (NACO 2007). 


\subsubsection{Zielsetzung und Methodik der Fallstudie A}

Zum besseren Einstieg in die Thematik der Fallstudie soll nach der Darstellung wichtiger Hintergrundinformationen hier nochmals die bereits unter 5.1 erläuterte Zielstellung der Fallstudie kurz wiederholt werden. Das Ziel der HIV-Studie Safety and Immunogenicity Study of tgAACO9, a Gag-PR-RT AAV HIV Vaccine war es, die Sicherheit und Immunogenität der HIV-Impfung tgAAC09 an gesunden Erwachsenen zu testen (Mehendale et al. 2008, S. 168).

Klinische Studien zur Testung neuer Medikamente oder Therapieoptionen durchlaufen 4 Phasen. Bei der Fallstudie handelt sich um eine Phase I-Studie. Das bedeutet, dass in dieser Studie eine neue biomedizinische Intervention an einer kleinen Personengruppe (beispielsweise 20-80 ProbandInnen) getestet wurde, um in erster Linie die Sicherheit der Intervention bewerten zu können. Bei der Evaluierung der Sicherheit in einer Phase I-Studie geht es beispielsweise, um das Prüfen der Verträglichkeit und Sicherheit bei der Verabreichung verschieden hoher Dosen und um das Identifizieren von Nebenwirkungen (WHO 2014).

Zur Methodik der Studie lässt sich festhalten, dass es sich um eine randomisierte und doppelt blinde Placebo-kontrollierte Studie handelte. Die StudienteilnehmerInnen wurden in drei Gruppen eingeteilt, die jeweils eine unterschiedlich hohe Dosis der Impfung beziehungsweise des Placebos erhielten. Innerhalb dieser drei Gruppen wurden die ProbandInnen per Zufall in die Impf- oder Placebogruppe eingeteilt und dies mit einem Verhältnis von 8:2. Weder das Forscherteam noch die ProbandInnen wussten, wer die Impfung oder das Placebo erhalten wird. Die Einteilung der ProbandInnen in die unterschiedlichen Dosisgruppen war allerdings bekannt. Für die Studie wurde eine Impfung verwendet, die gegen den HIV-1 Subtyp C wirksam sein soll, der häufigste HIV-Typ in Indien (Mehendale et al. 2010, S. 173). Die Impfung tgAAC09 enthält Gene des HIV-1 Subtyp C, die für bestimmte virale Enzyme, nämlich die Protease und Teile der Reversen Transkriptase und für Gag, ein Gen des HIVirus, kodieren (Mehendale et al. 2010, S. 169). Zusammengefasst werden diese Gene Gag-PR- $\Delta R T$ genannt und sind in einer Proteinkapsel eines rekombinanten (künstlich hergestellten) Adenoassoziierten Virus mit dem Serotyp 2 (rAAV2), der als Vektor dient, eingeschlossen (Mehendale et al. 2010, S. 169). Folglich wurde bei der Studie der Ansatz einer genetischen Immunisierung mithilfe eines Vektors verfolgt. Der Impfstoff wurde zuvor an Affen getestet und führte hier sowohl zu T-ZellAntworten als auch zur Bildung von Antikörpern und galt somit als vielversprechende Ausgangslage für die Studie mit Menschen (Mehendale et al. 2008, S. 873). Als Placebo wurde eine isotonische Salzlösung verwendet, die auch in der Impflösung enthalten war. Um die Sicherheit der Impfung zu prüfen, wurden die StudienteilnehmerInnen direkt nach der Injektion überwacht und sowohl lokale als auch systemische Reaktionen aufgezeichnet. Zusätzlich wurden unerwünschte Ereignisse, sogenannte 
adverse events (AE), und schwerwiegende unerwünschte Ereignisse, sogenannte serious adverse events (SAE), erfasst. Im Verlauf der Studie wurden die StudienteilnehmerInnen außerdem mehrfach auf HIV-1 und HIV-2 getestet. Die Tests wurden mit Enqyme-linked Immunosorbent Assays (ELISA) durchgeführt. Um die Immunogenität der Impfung festzustellen, wurde ein sogenanntes IFN- $\gamma$ secretory Ensyme Linked Immuno Spot Assay (ELISPOT) verwendet. Mit ELISPOT Assays kann man die Anzahl von Zellen messen, die spezifische Antikörper sezernieren (Czerkinsky et al. 1983) und hierdurch die Immunantwort auf die Impfung bestimmen.

Eine detailliertere Beschreibung der Methodik ist im Kontext dieser Arbeit nicht zielführend, jedoch sollen die Studienbedingungen und Informationen zu den StudienteilnehmerInnen und deren Lebenssituation genauer vorgestellt werden.

\subsubsection{Bedingungen und StudienteilnehmerInnen der Fallstudie A}

Die Studie wurde von der International AIDS Vaccine Initiative (IAVI) finanziert (Mehendale et al. 2008, S. 879). Die IAVI ist eine Nichtprofit-Organisation, die 1996 gegründet wurde und das Ziel verfolgt, eine präventive HIV-Impfung zu entwickeln, die weltweit zugänglich sein soll. Finanziell unterstützt wird IAVI von zahlreichen Regierungen, beispielsweise den USA, Schweden und Japan, als auch von Privatpersonen, Stiftungen und durch Kooperationen mit verschiedenen Pharmafirmen (IAVI: International AIDS Vaccine Initiative 2013). Der in der Studie getestete Impfstoff wurde von der Pharmafirma Targeted Genetics Corporation zur Verfügung gestellt (Jesani und Coutinho 2007a). Durchgeführt wurde die Studie im National AIDS Research Institute (NARI) in Pune, Indien, das 1992 gegründet wurde (NARI: National AIDS Research Institute 2010).

Eingeschlossen in die Studie wurden:

- gesunde Männer und Frauen im Alter zwischen 18 und 50 Jahren

- verfügbar für die geplanten Nachuntersuchungen für die gesamte Zeit der Studiendauer

- fähig informed consent zu erteilen

- kein Bericht über hoch riskantes Verhalten bezüglich HIV; bereit sich HIV-Tests zu unterziehen und die Ergebnisse zu erhalten

- falls sexuell aktiv, bereit Kondome zu verwenden von Beginn der Studie bis vier Monate nach der Impfung, wobei zusätzliche Verhütungsmittel erlaubt/erwünscht (ClinicalTrials.gov 2007)

Als Ausschlusskriterien für eine Studienteilnahme werden unter anderem folgende genannt:

- Immunschwäche oder Einnahme von Immunsuppressiva

- chronische Erkrankung oder auffällige Laborwerte

- Teilnahme an einer HIV-Impfstudie in der Vergangenheit 
- Schwangerschaft oder Plan, innerhalb der vier Monate nach der Impfung schwanger zu werden oder stillen

- Teilnahme an einer anderen klinischen Studie während der Studienteilnahme oder innerhalb zwölf Wochen nach der Impfung

- bestätigte HIV-1 oder HIV-2 Infektionen (ClinicalTrials.gov 2007)

Die Studie wurde in Indien mit insgesamt 30 ProbandInnen durchgeführt. Hierunter waren 16 männliche und 14 weibliche Studienteilnehmer. Das mittlere Alter der ProbandInnen betrug 34 Jahre, wobei 14 ProbandInnen jünger als 30 Jahre waren, acht zwischen 31 und 40 und acht zwischen 41 und 50 Jahre alt. Die Verteilung des Alters hinsichtlich männlicher und weiblicher Studienteilnehmer war ungefähr gleich (Mehendale et al. 2010, S. 171). Hinsichtlich des Bildungsstatus der ProbandInnen ist zunächst festzuhalten, dass keine AnalphabetInnen an der Studie teilnahmen. Vier der männlichen und acht der weiblichen StudienteilnehmerInnen hatten die Schule bis hin zur zehnten Klasse besucht. Des Weiteren besuchten acht Männer und fünf Frauen die Oberstufe und weitere vier Männer und eine Frau hatten Abitur. Wertet man Oberstufe und Abitur als höheren Schulabschluss, bedeutet dies, dass 75\% der Männer, aber nur 43\% der Frauen, die an der Studie teilnahmen, einen höheren Schulabschluss haben (Mehendale et al. 2010, S. 171). Die berufliche Situation der Probandinnen betreffend zeigen sich ebenfalls einige Unterschiede zwischen männlichen und weiblichen Studienteilnehmern. Zu der Kategorie festangestellt zählte der Großteil der Männer, nämlich insgesamt neun, allerdings nur eine Frau. Jeweils drei der Männer gaben an, selbstständig tätig zu sein oder in einer Fabrik zu arbeiten. Ein Mann und zwei Frauen waren im Gesundheitsbereich tätig. Die große Mehrheit, der an der Studie teilnehmenden Frauen, insgesamt elf, waren Sozialarbeiterinnen. Zur familiären Situation der StudienteilnehmerInnen stehen nur Angaben zum Familienstand der Personen zur Verfügung. Verheiratet waren demnach elf der Männer und 13 der Frauen und unverheiratet fünf der Männer und eine Frau (Mehendale et al. 2010, S. 171).

Als Nutzen für die StudienteilnehmerInnen kann gelten, dass diese für den Zeitraum der Studien versichert wurden. Des Weiteren wurden die ProbandInnen darüber aufgeklärt, dass sie für jegliche mit der Studie assoziierten gesundheitlichen Probleme eine vollständige Behandlung erhalten werden. Zusätzlich wurden sie darüber informiert, dass sie bei Problemen jederzeit, auch in Zukunft, das Forschungsinstitut kontaktieren könnten und ihnen eine angemessene gesundheitliche Versorgung bereitgestellt würde (Mehendale et al. 2010, S. 170). Darüber hinaus wurden die StudienteilnehmerInnen während ihrer Aufenthalte im Forschungsinstitut bezüglich HIV-Tests, Verhütung und der Reduzierung des Risikos einer HIV-Infektion beraten (Mehendale et al. 2010, S. 170). Als Risiko bei der Studienteilnahme sind mögliche Nebenwirkungen des Impfstoffes zu nennen. Dies ist insofern von besonderer Bedeutung, da es sich um eine Phase I-Studie handelte und somit noch keine vorherigen Informationen über potenzielle Nebenwirkungen beim Menschen vorliegen.

Eine genauere Betrachtung des Nutzens und der Risiken, beziehungsweise Nachteile einer 
Studienteilnahme, werden im Interpretationsteil der Fallstudie erläutert, da hier eine Beurteilung des Nutzen-Risiko-Verhältnisses folgen wird.

\subsubsection{Verlauf und Ergebnisse der Fallstudie A}

Laut AutorInnen besuchte das Forscherteam zur Vorbereitung der Studie den Standort einer noch laufenden HIV-Impfstudie in Thailand. Zudem wurde das Forschungszentrum NARI eigens für die Studie aufgebaut. Zusätzlich erhielten die ForscherInnen eine spezielle Ausbildung in den Bereichen Rekrutierungsprozesse, Forschung mit Menschen und gute klinische und labortechnische Praxis, die sogenannten good clinical and laboratory practices. Darüber hinaus wird angegeben, dass die ForscherInnen auch eine Einweisung hinsichtlich geschlechtsspezifischer Aspekte erhielten (Mehendale et al. 2010, S. 169). Allerdings gibt es keine genaue Erläuterung über den Inhalt dieser Einweisung oder für die Gründe das Forscherteam auf diesbezügliche Aspekte vorzubereiten. Vor Beginn der Studie unterzeichneten die National AIDS Control Organisation (NACO), der Indian Council of Medical Research (ICMR) und die International AIDS Vaccine Initiative (IAVI) eine Vereinbarung. Diese war die Grundlage für die Erarbeitung eines Antrages zur Durchführung der Studie. Der Antrag wurde vom National Advisory Board on HIV evaluiert und genehmigt. Außerdem wurde die Durchführung der Studie vom Institutional Ethics Committee, vom Drugs Controller General of India (DCGI), vom Genetic Engineering Approval Committee des Ministry of Environment and Forests, vom Health Ministry Screening Committee, von der Regierung Indiens, dem Scientific Advisory Sub-committee eingesetzt vom ICMR und vom Central Ethics Committee des ICMR genehmigt (Mehendale et al. 2010, S. 169).

Die Studie begann im Januar 2005 und wurde im Dezember 2006 abgeschlossen. Der Beginn des Rekrutierungsprozesses der ProbandInnen bleibt in den Publikationen leider unklar. In der Publikation zum Studienteil, der in Indien durchgeführt wurde, wird nur angegeben, dass potenzielle Freiwillige über sogenannte community sensitization gefunden wurden, was jedoch nicht genauer erläutert wird (Mehendale et al. 2010, S. 170). Zu Beginn der Rekrutierung nahmen 80 Freiwillige an einem Auswahlverfahren teil und hiervon wurden 40 als geeignete ProbandInnen eingestuft. Letztendlich wurden von diesen Freiwilligen 30 als ProbandInnen in die Studie aufgenommen (Mehendale et al. 2010, S. 170). Das Forscherteam und eine Gruppe von 20 (nicht näher bezeichneten) Experten in Indien entwickelten Formulare, zum einen für den informed consent des Auswahlverfahrens und zum anderen für die eigentliche Teilnahme an der Studie (Mehendale et al. 2010, S. 170). Zusätzlich wurde ein Verständnistest entwickelt, der überprüfen soll, ob die potenziellen ProbandInnen alle wichtigen studienrelevanten Informationen verstanden haben. Bei der Rekrutierung wurden von den Freiwilligen zunächst die Einwilligungsformulare für das Auswahlverfahren unterzeichnet und daraufhin das Auswahlverfahren durchgeführt. Hier wurden die bereits oben genannten Ein- und Ausschlusskriterien 
angewendet. An Personen, die als geeignet befunden wurden und bereit waren, für die eigentliche Rekrutierung zurückzukehren, wurde das Einwilligungsformular für die Teilnahme an der Studie ausgeteilt. Diese Personen mussten daraufhin den entwickelten Verständnistest absolvieren. Potenzielle ProbandInnen, die den Test bestanden hatten und die Einwilligungsformulare unterzeichneten, wurden als StudienteilnehmerInnen rekrutiert (Mehendale et al. 2010, S. 170). Im Rahmen des informed consentProzesses wurde den ProbandInnen versichert, dass jegliche Informationen im Rahmen ihrer Studienteilnahme vertraulich sind und diese nicht an unbefugte Personen weitergegeben würden (Mehendale et al. 2010, S. 170).

Die 30 ProbandInnen wurden in drei verschiedene Gruppen à zehn Personen eingeteilt, die jeweils eine unterschiedliche Dosis der Impfung erhalten sollten. Innerhalb dieser Gruppen wurden jeweils acht ProbandInnen in die Impfgruppe und zwei ProbandInnen in die Placebogruppe eingeteilt, wobei weder das Forscherteam noch die ProbandInnen wussten, in welche Gruppe sie eingeteilt waren, da es sich um eine doppelt blinde Studie handelte (Mehendale et al. 2010, S. 170).

Zunächst wurde die Niedrigdosisgruppe geimpft. Anschließend wurde von einem externen Ausschuss, dessen Mitglieder durch die Studiensponsoren eingesetzt wurden, die Sicherheit der Impfung bewertet, bevor die ProbandInnen in der Mitteldosisgruppe geimpft wurden. Dasselbe Vorgehen wurde auch vor der Impfung der ProbandInnen der Hochdosisgruppe angewendet (Mehendale et al. 2010, S. 170). Alle StudienteilnehmerInnen wurden einmalig geimpft oder erhielten einmalig das Placebo. Die ProbandInnen erhielten nach dem Auswahlverfahren und der Rekrutierung noch zehn Nachsorgeuntersuchungen, die verteilt über zwölf Monate stattfanden. In der Woche nach der Rekrutierung wurden zusätzlich zwei Telefongespräche zwischen den ProbandInnen und den ForscherInnen geführt (Mehendale et al. 2010, S. 170). Den StudienteilnehmerInnen wurden bei der Erstvorstellung, am Tag der Einschreibung in die Studie und in Woche zwei, vier, zwölf, 24, 36 und 52 Blutproben entnommen. Die Blutproben wurden verwendet, um die Immunogenität der Impfung zu überprüfen. Des Weiteren wurden die Blutproben in Woche null, vier, zwölf, 24, 36 und 52 auf HIV-1 und HIV-2 getestet (Mehendale et al. 2010, S. 170). Für die Prüfung der Sicherheit der Impfung wurden die StudienteilnehmerInnen über vier Stunden nach der Injektion in der Klinik überwacht und die Vitalparameter aufgezeichnet, um mögliche Sofortreaktionen zu erfassen. Adverse events (AEs) wurden im Hinblick auf die Frage, ob diese mit der Impfung in Zusammenhang stehen, in folgende Kategorien eingeteilt: kein Zusammenhang, möglicherweise, wahrscheinlich oder definitiv ein Zusammenhang zwischen AE und Impfung (Mehendale et al. 2010, S. 170). Die Ergebnisse bezüglich der Sicherheit und der Immunogenität der Impfung wurden sowohl zwischen den drei Dosisgruppen als auch zwischen den ProbandInnen der Impfgruppen und der Placebogruppen verglichen (Mehendale et al. 2010, S. 171). Alle 30 rekrutierten ProbandInnen beendeten die Studie und nahmen folglich an allen vorgeschriebenen Nachsorgeuntersuchungen teil (Mehendale et al. 2010, S. 171). 


\section{$\underline{\text { Studienergebnisse }}$}

Im folgenden Abschnitt sollen nun die Ergebnisse bezüglich der Sicherheit und Immunogenität der Impfung dargestellt werden. Innerhalb von sieben Tagen nach der Injektion wurden insgesamt sechs lokale Reaktionen von vier StudienteilnehmerInnen gemeldet. Die lokalen Reaktionen wurden als dosisabhängig eingestuft. Schmerz war die am häufigsten gemeldete lokale Reaktion und musste bei einem/r der ProbandInnen mit Schmerzmittel behandelt werden (Mehendale et al. 2010, S. 170). Unter den systemischen Reaktionen wurde am häufigsten Fieber, Kopfschmerzen, Muskelschmerzen und Unwohlsein genannt. Insgesamt wurden 14 systemische Reaktionen von fünf ProbandInnen aus der Impfgruppe und von einer Person aus der Placebogruppe aufgezeichnet. Eine/r der ProbandInnen benötigte medizinische Behandlung. Alle systemischen Reaktionen wurden als nicht im Zusammenhang stehend zur Impfung eingestuft (Mehendale et al. 2010, S. 171). Bis zu 24 Wochen nach der Injektion wurden insgesamt 45 AEs dokumentiert. Diese traten bei 19 von 24 (79\%) der ProbandInnen der Impfgruppe und bei vier von sechs $(66,7 \%)$ der ProbandInnen der Placebogruppe auf. Der Unterschied sowohl zwischen den AEs in der Impfgruppe und in der Placebogruppe als auch zwischen den unterschiedlichen Dosisgruppen erwies sich statistisch als nicht signifikant. AEs wurden von 69\% der männlichen und von 86\% der weiblichen Studienteilnehmer gemeldet (Mehendale et al. 2010, S. 171). Die Einteilung der AEs in Schweregrade ergab, dass 51\% als mild, 34\% als mäßig und 13\% als ernsthaft eingestuft wurden. Ein AE wurde als sehr ernsthaft bewertet. Hierzu gibt es jedoch keine genaueren Angaben. 78\% der AEs mussten behandelt werden. Drei der AEs (7\%) wurden als möglicherweise mit der Impfung in Zusammenhang stehend eingestuft. Keines der AEs wurde als wahrscheinlich oder definitiv mit der Impfung in Zusammenhang stehend bewertet. Die am häufigsten dokumentierten AEs waren Erkrankungen des Bewegungsapparates und des Atmungssystems. Des Weiteren waren 7\% der AEs auffällige Laborwerte, die sich jedoch jeweils bei einer wiederholten Analyse normalisiert hatten und weitere $7 \%$ waren traumatisch bedingte Verletzungen. Insgesamt wurden viele AEs dokumentiert, nämlich von 76\% der 30 Studienteilnehmerinnen (Mehendale et al. 2010, S. 172). Allerdings wurde die Mehrzahl der AEs im Rahmen der vereinbarten Nachsorgeuntersuchungen gemeldet und nur 38\% der AEs wurden von den StudienteilnehmerInnen selbstständig, unabhängig von den vereinbarten Terminen in der Klinik, gemeldet. Die ForscherInnen erläutern, dass bei einer Studie, bei der zu einem heiklen Thema wie HIV geforscht wird, die ProbandInnen möglicherweise Symptome wahrnehmen und angeben, die ihnen im normalen Alltag nicht zwingend aufgefallen wären (Mehendale et al. 2010, S. 173). Während der gesamten Studienlaufzeit traten zwei SAEs auf, die als diese eingestuft wurden, weil die betroffenen StudienteilnehmerInnen hospitalisiert werden mussten. Hierbei handelte es sich zum einen um eine akute Panikattacke und zum anderen um einen Bandscheibenvorfall. Beide SAEs wurden als nicht in Zusammenhang mit der Impfung eingeordnet, da zum einen die Ursachen der SAEs nicht mit der Impfung erklärbar waren und zum anderen kein zeitlicher Zusammenhang bestand (Mehendale et al. 2010, S. 172). 
Zusammenfassend stufen die ForscherInnen die getestete Impfung in allen drei Dosierungsstufen als sicher und gut verträglich für Menschen ein (Mehendale et al. 2010, S. 173).

Für die Feststellung der Immunogenität der Impfung wurden sowohl gefrorene als auch frisch isolierte Blutzellen aus den Blutproben der ProbandInnen verwendet. Eine Immunantwort auf die Impfung konnte per Messung mit IFN- $\gamma$ ELISPOT bei nur einem/r der acht ProbandInnen aus der Hochdosisimpfgruppe nachgewiesen werden und diese Immunantwort stellte sich zudem als nur mäßig dar. Nachgewiesen wurde die Immunantwort erstmals in Woche 24 und blieb auch in Woche 36 und 52 bestehen, allerdings mit einem abnehmenden Ausmaß. Zusätzlich wurde eine Immunantwort bei jeweils einem/r Probanden/in aus der Niedrig- und Mitteldosisgruppe nachgewiesen. Allerdings lagen diese Immunantworten jeweils nur knapp über der Nachweisgrenze (Mehendale et al. 2010, S. 172). Zudem konnten bei keinem der ProbandInnen Antikörper gegen das virale Gen gag nachgewiesen werden (Mehendale et al. 2010, S. 173). Insgesamt lässt sich festhalten, dass die Studie hinsichtlich der Sicherheit und Verträglichkeit der Impfung laut der AutorInnen zwar ein positives Ergebnis verzeichnen kann, jedoch die erwünschte Induktion einer starken Immunantwort ausblieb (Mehendale et al. 2010, S. 173). 


\subsection{Interpretation der Fallstudie A im Hinblick auf Vulnerabilität der}

\section{StudienteilnehmerInnen}

Nach der Vorstellung der HIV-Impfstudie Safety and Immunogenicity Study of tgAAC09, a Gag-PR-RT $A A V H I V$ Vaccine gilt es nun zunächst anhand von Zusatzinformationen und offen gebliebenen Fragen hinsichtlich ethischer Aspekte eine normative Bewertung der Studiendurchführung vorzunehmen (Kapitel 5.2.1). Die Bewertung dieser ethischen Aspekte soll im Anschluss dazu dienen, eine mögliche Vulnerabilität der StudienteilnehmerInnen anhand der in Kapitel 3.4 aufgestellten Vulnerabilitätskriterien zu überprüfen (Kapitel 5.2.2).

\subsubsection{Ethische Aspekte bei der Durchführung von Fallstudie A}

Wie bereits erwähnt, wurde die Studie nicht nur in Indien, sondern auch in Deutschland und Belgien durchgeführt. Insgesamt nahmen an der Studie in Europa 50 ProbandInnen teil. Im Unterschied zur Studie in Indien erhielten 21 der StudienteilnehmerInnen in Europa eine zusätzliche zweite Injektion mit dem Impfstoff beziehungsweise Placebo (Mehendale et al. 2008, S. 873). Bezüglich der Ergebnisse der Studie in Europa und Indien zeigen sich sowohl die Sicherheit als auch die nur mäßig induzierte Immunantwort betreffend keine bedeutenden Unterschiede (Vgl. Mehendale et al. 2008 und Mehendale et al. 2010). Folglich scheint die Wirksamkeit des Impfstoffs eher gering zu sein und dies, obwohl manche ProbandInnen eine zweite Impfung erhielten. Auffällig ist, dass der informed consent-Prozess bezüglich der Studie in Europa wesentlich weniger ausführlich beschrieben wird. Ein Verständnistest, wie er in Indien durchgeführt wurde, bleibt hinsichtlich der Studie in Europa unerwähnt. Zudem gab es anscheinend für die ProbandInnen in Europa im Gegensatz zu den ProbandInnen in Indien keine zusätzlichen Einwilligungsformulare für das Auswahlverfahren vor der eigentlichen Rekrutierung. Darüber hinaus wurden über die StudienteilnehmerInnen in Europa im Vergleich zu denen in Indien weniger Zusatzinformationen veröffentlicht, wie beispielsweise der Bildungsstatus oder die aktuelle berufliche Situation. Sowohl die zusätzlichen Maßnahmen im Rahmen des informed consent als auch die detailliertere Beschreibung der sozioökonomischen Situation der ProbandInnen in Indien im Vergleich zur Publikation hinsichtlich der Studie in Europa, lässt die Vermutung zu, dass die ForscherInnen bei den indischen StudienteilnehmerInnen stärkere Vorsichts- und Schutzmaßnahmen veranlassten. Dies könnte wiederum durch die Annahme des Forscherteams begründet sein, möglicherweise mit einer vulnerablen Gruppe zu forschen.

Des Weiteren konnte recherchiert werden, dass der Impfstoff tgAAC09 nach der Studie in Indien zudem in einer Phase II-Studie in Uganda, Südafrika und Sambia getestet wurde (Vardas et al. 2010). Hierbei erhielten die insgesamt 91 ProbandInnen jeweils eine zweite Impfung beziehungsweise ein 
Placebo. Wie bereits bei der Studie in Indien wurden die ProbandInnen der Phase II-Studie in drei verschiedene Dosisgruppen eingeteilt, wobei die Dosen im Vergleich zur Phase I-Studie um eine Stufe erhöht wurden und somit die höchst möglich praktikable Dosis erreicht wurde (Vardas et al. 2010, S. 941). Die im Jahre 2010 veröffentlichten Ergebnisse zeigen, dass auch bei der Verabreichung des Impfstoffs in höheren Dosen keine Bedenken bezüglich der Sicherheit und Verträglichkeit für Menschen entstehen. Zudem wurde festgestellt, dass eine höhere Dosis mit einer verbesserten Immunantwort einhergeht. Als Schlussfolgerung geben die ForscherInnen allerdings an, dass die Immunogenität des Impfstoffs aus ungeklärten Gründen weiterhin nur mäßig sei und keine weitere Forschung hierzu geplant sei (Vardas et al. 2010, S. 941). Demnach wäre ein möglicher Nutzen für die ProbandInnen der Fallstudie, im Sinne einer hohen Wirksamkeit der Impfung und folglich ein Schutz vor einer HIV-Infektion, als sehr gering einzuschätzen.

Die zu analysierende Studie war die erste in Indien durchgeführte Phase I HIV-Impfstudie. Excler et al. ${ }^{8}$ veröffentlichten 2008 einen Artikel, in dem sie erläutern, welche Vorbereitungen in Indien getroffen wurden, um gewisse wissenschaftliche als auch ethische Mindeststandards für HIV-Impfstudien in Indien zu setzen (Excler et al. 2008). Die AutorInnen beziehen sich hierbei zum einen im Allgemeinen auf HIV-Impfstudien in Indien und zum anderen konkret auf die bereits vorgestellte Fallstudie. So gab es beispielsweise die Befürchtung, dass durch vorherige unethische Studien in Indien und aufgrund von Ignoranz, Stigmatisierung und Diskriminierung hinsichtlich HIV und AIDS ein gewisses Misstrauen in der Bevölkerung entstanden sei und es hierdurch schwierig sein würde, Freiwillige für HIV-Impfstudien zu rekrutieren (Excler et al. 2008, S. 532). Hinsichtlich der Fallstudie beschreiben Excler et al., dass zur Vorbereitung der Studie eng mit den lokalen Gemeinden zusammengearbeitet wurde, um solchen Befürchtungen vorzubeugen (Excler et al. 2008, S. 532). Des Weiteren wurde ein sogenanntes Gender Advisory Board mit unabhängigen Experten gegründet, die zu geschlechtsspezifischen Themen arbeiten und hierbei die Rechte von Frauen und diesbezügliche gesundheitliche Aspekte in den Fokus rücken. Dieser Ausschuss hatte die Aufgabe die ForscherInnen hinsichtlich geschlechtsspezifischer Themen zu schulen. Zudem wurden die ForscherInnen durch das Gender Advisory Board bei der Ausarbeitung von informed consent Dokumenten, die eine geschlechtsspezifische Perspektive miteinbeziehen, unterstützt (Excler et al. 2008, S. 533). Hierbei wurde unter anderem berücksichtigt, dass Frauen möglicherweise bezüglich Entscheidungsfindungen in ihrer Autonomie eingeschränkt sein könnten. Ein weiteres Thema waren die möglichen Auswirkungen für Frauen, wie beispielsweise Stigmatisierung, Schuldzuweisungen und der Verlust finanzieller Unterstützung, wenn die Vertraulichkeit bezüglich ihrer Studienteilnahme gebrochen würde (Excler et al. 2008, S. 533). Zur Vorbereitung auf HIV-Impfstudien in Indien wurde darüber hinaus eine Gruppe von Experten herangezogen, die dabei helfen sollte, den Prozess und die Dokumente des informed consent speziell für die lokalen Bedingungen auszuarbeiten. Als

\footnotetext{
${ }^{8}$ Jean-Louis Excler und Sonli Kochhar gehören sowohl zu den AutorInnen des o.g. Artikels (Excler et al. 2008) als auch der Publikationen der Fallstudie (Mehendale et al. 2008 und Mehendale et al. 2010)
} 
Erfolg nach Abschluss der ersten HIV-Impfstudie in Indien erläutern Excler et al., dass Freiwillige aus allen sozioökonomischen und Bildungskontexten der Gesellschaft und dass Frauen und Männer in einem ausgeglichenen Verhältnis rekrutiert werden konnten. Sie heben als positiv hervor, dass hierdurch die Vorurteile abgebaut werden konnten, Frauen in Indien würden aufgrund gesellschaftlichen Drucks nicht an klinischen Studien teilnehmen können oder Personen aus einer höheren sozioökonomischen Klasse wären nicht bereit dazu, (Excler et al. 2008, S. 536).

Hinsichtlich ethischer Aspekte bei der Durchführung der Fallstudie wurden 2007 im Indian Journal of Medical Ethics sowohl positive als auch kritische Punkte diskutiert. Zusätzlich wurden jeweils die Stellungnahmen des IAVI, also des Sponsors der Studie, veröffentlicht. Jesani und Coutinho, die Autoren, die die ethische Diskussion über die Fallstudie anstießen, nennen zunächst Aspekte, die aus ethischer Sicht positiv $\mathrm{zu}$ bewerten sind. Durch die Fallstudie würden gewisse ethische Mindeststandards für zukünftige HIV-Studien in Indien gesetzt (Jesani und Coutinho 2007b, S. 2). Sie heben als positiv hervor, dass die ForscherInnen vor Beginn der Studie bezüglich des informed consents von Experten beraten wurden (vgl. Excler et al. 2008). Zudem begrüßen sie den Versuch, die lokale Gemeinschaft mithilfe sogenannter community advisory boards stärker miteinzubeziehen. Zusätzlich erläutern sie, dass über alle bekannten Risiken und vor allem über das Recht die Einwilligung zur Studienteilnahme jederzeit widerrufen zu können, aufgeklärt wurde. Des Weiteren wurde bei der Rekrutierung auf ein ausgeglichenes Geschlechterverhältnis geachtet und versucht auszuschließen, dass potenzielle ProbandInnen unter Zwang zustimmen. Darüber hinaus wird von den Autoren der Verständnistest im Rahmen des informed consent-Prozesses als positiv bewertet (Jesani und Coutinho 2007b, S. 2). Um die Möglichkeit auszuschließen, dass StudienteilnehmerInnen aufgrund eines unangemessenen finanziellen Anreizes zustimmen, wurde die Höhe der Aufwandsentschädigung standardisiert für alle ProbandInnen festgelegt (Jesani und Coutinho 2007b, S. 2f). Wie die Aufwandsentschädigung festgelegt wurde und die Höhe der selbigen bleibt jedoch unklar. Diesbezügliche Informationen wurden weder in den Publikationen zur Fallstudie noch in dem Artikel von Jesani und Coutinho (2007b) veröffentlicht. Für ProbandInnen, die sich während der Studie mit HIV infizieren würden, versicherte man den freien Zugang zu gesundheitlicher Versorgung und Therapie über fünf Jahre. Allen ProbandInnen wurde außerdem zugesichert, dass sie für jegliche mit der Studie assoziierten gesundheitlichen Probleme medizinische Behandlung und Entschädigungen erhalten würden (Jesani und Coutinho 2007b, S. 3). Jesani und Coutinho erläutern, dass es zwar keine unabhängige Begutachtung über die tatsächliche Umsetzung dieser Vorhaben und Standards gab, die Tatsache, dass jedoch alle involvierten ForscherInnen diesen Standards zustimmten, setze allerdings bereits wichtige ethische Maßstäbe für in Indien stattfindende Studien (Jesani und Coutinho 2007b, S. 3). Jesani und Coutinho führen allerdings auch drei Kritikpunkte zur analysierten HIV-Impfstudie an. 


\section{Zusammenarbeit zwischen IAVI und dem Forscherteam und ProbandInnen in Indien}

Der erste Kritikpunkt betrifft ethische Bedenken, die die Zusammenarbeit zwischen IAVI und dem Forscherteam in Indien und den dortigen ProbanInnen betreffen. Jesani und Coutinho erläutern die Grundlage für diese Bedenken folgendermaßen:

„Phase I trials of the tgAAC09 vaccine had started in December 2003 in Belgium and Germany and their results were awaited in early 2005. Instead of waiting for these results, a trial of the same candidate vaccine, with the same protocol, was started in India on February 7, 2005. On February 22, barely two weeks after the trial was launched in India, Targeted Genetics, the company conducting the trials in collaboration with the International AIDS Vaccine Initiative (IAVI), issued a press statement announcing the preliminary results of the Belgium-Germany trials" (Jesani und Coutinho 2007b, S. 3). ${ }^{9}$

Dies bedeutet, dass die Studie in Indien begonnen wurde, ohne die Ergebnisse aus Deutschland und Belgien abzuwarten, obwohl anzunehmen ist, dass diese der IAVI bereits vorlagen. Nun kritisieren die Autoren, dass nicht ersichtlich sei, ob die Studienergebnisse aus Europa mit dem Studienteam in Indien besprochen wurden. Dies zeige eine unzureichende Kommunikation zwischen dem Forscherteam in Europa und dem in Indien. Es wäre möglich, dass man das Studienprotokoll aufgrund der Ergebnisse aus Europa hätte ändern müssen, beispielsweise hätte es Hinweise auf gefährliche Nebenwirkungen geben können, die eventuell dazu veranlasst hätten, die Studie in Indien gar nicht erst zu beginnen (Jesani und Coutinho 2007b, S. 3). Da es weder bei der Studie in Europa noch bei der Studie in Indien, Anhaltspunkte für schwerwiegende unerwünschte Nebenwirkungen gab, trifft diese Befürchtung in diesem Fall nicht zu. Jesani und Coutinho kritisieren jedoch zu Recht, dass man die potenziellen ProbandInnen in Indien im Rahmen des informed consent-Prozesses über die bereits vorliegenden Ergebnisse aus Europa hätte aufklären müssen. Es wäre nämlich möglich, dass dies ihre Entscheidung für oder gegen eine Studienteilnahme beeinflusst hätte (Jesani und Coutinho 2007a, S. 112). Ein weiterer möglicher Kritikpunkt in diesem Kontext wäre, dass man die ProbandInnen in Indien unnötigen Risiken und Unannehmlichkeiten aussetzt, da der Impfstoff bereits getestet wurde. Letzteres wird von der IAVI insofern zurückgewiesen, da der getestete Impfstoff speziell für den HIV Subtyp entwickelt wurde, der in Asien und Afrika am häufigsten ist. Somit war es für die indischen Behörden und IAVI wichtig, die Sicherheit und Verträglichkeit auch an indischen ProbandInnen zu testen (Nayyar 2007). Zudem wird von der IAVI erläutert, dass erst nach Beginn der Studie in Indien erste Ergebnisse aus Europa vorlagen, und diese seien daraufhin dem indischen Forscherteam zur Verfügung gestellt worden. Im Anschluss wurde laut IAVI anhand der Überprüfung wissenschaftlicher, behördlicher, ethischer und die Sicherheit der Impfung betreffender Aspekte beschlossen, die Studie in Indien fortzuführen. Zusätzlich gibt die IAVI an, dass die ProbandInnen in Indien über die Ergebnisse aus Europa informiert wurden (Nayyar 2007). Allerdings bleibt die Frage offen, ob die Ergebnisse aus Deutschland und Belgien der IAVI tatsächlich erst nach dem Studienbeginn in Indien vorlagen. Jesani und Coutinho antworten auf die Stellungnahme der IAVI, dass dies nicht der Fall sei. Sie begründen

\footnotetext{
${ }^{9}$ Originalquelle der Pressemitteilung von Targeted Genetics nicht verfügbar
} 
dies damit, dass es unmöglich sei, dass IAVI zu Beginn der Studie in Indien keine Ergebnisse aus Europa vorliegen hatte, wenn knapp zwei Wochen später Targeted Genetics, die nicht einmal Co-Sponsor für die Studie waren, bereits Ergebnisse als Pressemitteilung veröffentlichen. Sie erläutern, dass es sicherlich mehr als 15 Tage dauern würde, erste Ergebnisse zu analysieren und in eine Form zu bringen, die veröffentlicht werden kann und zudem würde es auch eine gewisse Zeit dauern, bis diese an die Pharmafirma weitergegeben werden und bis diese entschieden hat, ob sie die Ergebnisse veröffentlichen will (Jesani und Coutinho 2007a, S. 111). Folglich wird hier aufgrund der Aspekte, die Jesani und Coutinho vorbringen, davon ausgegangen, dass IAVI vor Beginn der Studie in Indien die Ergebnisse aus Europa vorlagen. Darüber hinaus äußern Jesani und Coutinho ethische Bedenken bezüglich der Zusammenarbeit von IAVI mit dem indischen Forscherteam im Vergleich zur Zusammenarbeit von IAVI mit Targeted Genetics. Jesani und Coutinho geben an, dass IAVI in einem persönlichen Gespräch bestätigte, dass das indische Forscherteam erst nachdem Targeted Genetics die Ergebnisse veröffentlicht hatte, über diese informiert wurde.

Sie kritisieren hierzu:

„Had IAVI given its collaboration with Indian researchers and the participants of the trial at least as much importance as it gave to its collaboration with the company (we believe that collaboration with researchers and concerns of participants should given more importance), it would have spared itself from doing wrong” (Jesani und Coutinho 2007a, S. 112).

\section{Unklarheiten bzgl. der Bereitstellung der getesteten HIV-Impfung für die indische Bevölkerung}

Der zweite von Jesani und Coutinho angeführte Kritikpunkt betrifft die Vereinbarungen zwischen der Pharmafirma, die den Impfstoff entwickelte und hierfür die Patentrechte hat, und der Regierung Indiens. Sie fordern hierzu, dass nur potenzielle Impfstoffe getestet werden sollten, wenn diese dem Gastland, in dem eine Studie durchgeführt wird, zu einem angemessenen Preis zur Verfügung gestellt würde. Folglich müssten gezielte Vereinbarung getroffen werden und ebenso Mechanismen der Haftung bei Nichteinhaltung, um sicherzustellen, dass ein Land von einer erfolgreich durchgeführten Studie profitiert (Jesani und Coutinho 2007b, S. 3). In der Stellungnahme der IAVI wird angegeben, dass sichergestellt wird, Impfstoffe in entwickelten und in Entwicklungsländern gleichzeitig zur Verfügung zu stellen. Weitere Angaben oder Informationen hierzu gibt es in der Stellungnahme nicht. Die Stellungnahme der IAVI und die analysierte Studie betreffend, kritisieren Jesani und Coutinho, dass die Vereinbarungen mit der indischen Regierung nicht ausreichend offengelegt wurden und keine genaueren Informationen vorliegen. Sie erläutern, dass Menschen in Indien ein Recht darauf hätten, über den potenziellen Nutzen nach Abschluss der Studie informiert zu werden, insbesondere im Hinblick auf die Patentrechte, den Verkauf und die Verwendung dieser Impfung in Indien (Jesani und Coutinho 2007a, S. 111). Folglich bleibt hier laut Jesani und Coutinho die Frage offen, warum diese Vereinbarungen nicht öffentlich gemacht wurden. 


\section{Fragwürdigkeit der lokalen Relevanz einer präventiven HIV-Impfung für Indien}

Als dritten Kritikpunkt an der analysierten Studie bemängeln Jesani und Coutinho, dass bei der Studie nur der Ansatz verfolgt wurde, eine präventive Impfung gegen HIV zu finden und die Möglichkeit einer therapeutischen Impfung außer Acht gelassen wurde (Jesani und Coutinho 2007b, S. 3). Die Entwicklung einer therapeutischen Impfung erläutern sie, sei zwar den wissenschaftlichen Aufwand betreffend mit einer präventiven vergleichbar. Aber es sei wesentlich kostspieliger eine ganze Bevölkerung mit einem Präventivimpfstoff $\mathrm{zu}$ versorgen, als nur bereits infizierte Personen therapeutisch zu impfen (Jesani und Coutinho 2007b, S. 3). Jesani und Coutinho (2007b, S. 3) geben zudem an, dass eine therapeutische Impfung aus ethischer Sicht weniger problematisch sei, erläutern dies jedoch nicht genauer. Die IAVI bezieht hierzu keine Stellung, was Jesani und Coutinho als verständlich bezeichnen, da es das Ziel von IAVI sei, eine präventive Impfung zu entwickeln (Jesani und Coutinho 2007a, S. 111).

An dieser Stelle ist es hilfreich, zusätzliche Informationen anzubringen, die den dritten Kritikpunkt betreffen. Thomas erläutert ethische Konflikte, die mit der ausschließlichen Entwicklung einer präventiven Impfung für Indien einhergehen (Thomas 2004). Er beschreibt, dass die Mehrheit der Menschen in Indien, die mit HIV infiziert sind, sich ihrer Verletzlichkeit und ihres HIV-Status nicht bewusst seien, viele in Armut lebten und ihnen eine grundlegende Gesundheitsversorgung vorenthalten werde (siehe hierzu Kapitel 4.2.2). Den dringenden Bedürfnissen dieser Menschen müsste im Rahmen der Forschung der Vorrang gelten und eine präventive Impfung würde für deren Lebensqualität vermutlich keine entscheidende Verbesserung bringen (Thomas 2004). Des Weiteren zeigten die Ergebnisse vorausgehender Studien, dass die Produktion und Verteilung einer präventiven Impfung noch Jahrzehnte dauern werde (Thomas 2004). Thomas widerspricht daher der Meinung vieler ForscherInnen, eine präventive Impfung sei langfristig die beste Hoffnung zur Kontrolle von HIV/AIDS. Er schlägt stattdessen vor, dass eine langfristige Prävention von HIV durch politisches Engagement im Sinne von Programmen, die die erhöhte Verletzlichkeit bestimmter Bevölkerungsgruppen reduzieren und sowohl nationale als auch globale Ungleichheiten in der Gesundheitsversorgung aufgreifen, erreicht werden könne. Außerdem erläutert er, dass eine erhöhte Anfälligkeit einer HIV-Infektion, abhängig vom Geschlecht und der sozioökonomischen Situation ungleich verteilt sei. Ein präventives Impfprogramm würde laut Thomas vor ähnlichen Hindernissen stehen und folglich die Personen, die Prävention am dringendsten benötigen, vermutlich am wenigsten erreichen (Thomas 2004). Eine therapeutische Impfung hingegen könne dazu beitragen, die Krankheitslast zu senken und die Lebensqualität von Personen, die mit HIV leben, zu erhöhen. Zusätzlich könne eine therapeutische Impfung eine sinnvolle Alternative zu ART sein, die benötigt wird, wenn bisherige Therapiemöglichkeiten aufgrund von Resistenzen weniger wirksam werden (Thomas 2004). Sahay und Mehendale entgegnen, dass die bisherigen Therapiestrategien von HIV ziemlich erfolgreich seien und 
es somit schwer vorstellbar sei, dass eine therapeutische Impfung hier eine bessere Alternative darstelle (Sahay und Mehendale 2004). Zusammenfassend lässt sich jedoch festhalten, dass es einige gute Gründe gibt gerade in Indien eher an einer therapeutischen als an einer präventiven Impfung zu forschen und unklar bleibt, warum diese Möglichkeit von IAVI nicht aufgegriffen wird.

Um eine mögliche Vulnerabilität der ProbandInnen der Fallstudie beurteilen zu können, sollen an dieser Stelle noch die Ergebnisse einer Studie vorgestellt werden, die das Wissen über HIV/AIDS und die Bereitschaft an einer HIV-Impfstudie teilzunehmen unter Hochrisikogruppen (Fernfahrer, MSM, IDU, Sexarbeiterinnen und Personen mit einer sexuell übertragbaren Erkrankung) und monogam lebenden verheirateten Frauen in Indien abfragte (Suhadev et al. 2006). Begründet wird der Einschluss verheirateter Frauen (insgesamt 21), in die oben genannte Studie, neben Personen, die einer Hochrisikogruppe bezüglich HIV angehören, dadurch, dass die Anzahl HIV-infizierter Frauen in Indien stetig steige (Suhadev et al. 2006). Die Studie wurde mit insgesamt 112 TeilnehmerInnen (60\% Männer) in Chennai und Madurai durchgeführt. Ein Vortest zu Beginn der Studie zeigte, dass das Wissen über HIV-Impfstudien unter den TeilnehmerInnen sehr gering war. Daraufhin wurden die StudienteilnehmerInnen diesbezüglich unterrichtet und beispielsweise sichergestellt, dass sie das Konzept einer Placebo-kontrollierten Studie verstehen (Suhadev et al. 2006). Im Anschluss wurden Fokusgruppendiskussionen durchgeführt und hierbei Fragen zu HIV-Impfungen, zu den Gründen sich für oder gegen eine Teilnahme an einer HIV-Impfstudie zu entscheiden und zum Einfluss einer HIVImpfung auf mögliches Risikoverhalten gestellt. Daraufhin wurden Einzelinterviews geführt und hierbei soziodemographische Daten (Alter, Geschlecht, Religion, Bildungsstatus, Einkommen) und mögliches Risikoverhalten der betreffenden Person abgefragt. 68\% der StudienteilnehmerInnen beendeten die mittlere Reife (Suhadev et al. 2006). Das Wissen (nach dem Unterrichten) über HIVImpfstudien betreffend, ergab die Studie, dass 90\% der StudienteilnehmerInnen Fragen hinsichtlich der Sicherheit und der Funktionsweise von HIV-Impfungen korrekt beantworteten. Ein signifikanter Unterschied zwischen den männlichen und weiblichen Studienteilnehmern zeigte sich bei einer Frage zu Placebo-kontrollierten Studien. Hier wussten 82\% der Männer und 100\% der Frauen, dass bei solchen Studien manche ProbandInnen einen Wirkstoff und manche ein Placebo erhalten würden. Insgesamt wussten nur wenige, dass den ProbandInnen nach der Studie mitgeteilt würde, ob sie das Placebo oder den Impfstoff erhielten (Suhadev et al. 2006). Zu den möglichen Vorteilen beziehungsweise Gründen sich für eine Teilnahme an einer HIV-Impfstudie zu entscheiden, gaben 98\% der Frauen an (Männer 90\%), dass sie hierdurch eine geringere Wahrscheinlichkeit hätten, sich mit HIV zu infizieren. Zudem stimmten 89\% der Frauen und 92\% der Männer zu, dass der Schutz vor einer HIV-Infektion ein weiterer Vorteil einer Studienteilnahme sei. Insgesamt glaubten 98\% der StudienteilnehmerInnen, dass ihre Bereitschaft, an einer HIV-Impfstudie teilzunehmen, wichtig für das Allgemeinwohl Indiens sei (Suhadev et al. 2006). Als Hürde an einer HIV-Impfstudie teilzunehmen, gaben 61\% die Unsicherheit bezüglich der Wirksamkeit des Impfstoffs an. Unsicherheit und 
Unbehagen wurde von 43\% angegeben und 32\% sorgten sich über mögliche Langzeitnebenwirkungen. Zudem gaben $50 \%$ als eine weitere Sorge an, dass sie nicht wüssten, wie eine Studienteilnahme ihr Leben beeinflussen würde. Zusätzlich äußerten 31\% die Sorge eine Studienteilnahme könnte Ehe, Beruf und das Abschließen einer Krankenversicherung negativ beeinflussen (Suhadev et al. 2006). Von besonderer Bedeutung für den Kontext dieser Arbeit ist zusammenfassend, dass Frauen bei dieser Studie eine höhere Bereitschaft zeigten, an einer HIV-Impfstudie teilzunehmen und der angegebene Grund hierfür war, dass sie sich hierdurch möglicherweise vor einer HIV-Infektion durch ihren Ehemann schützen könnten (Suhadev et al. 2006). Abschließend geben die AutorInnen an: „Altriusm was found to be a main motivation for participating in efficacy trials across all study populations“ (Suhadev et al. 2006, S. 637). Sie erläutern jedoch, dass das Wissen über HIV-Impfstudien insgesamt zu gering sei und diesbezügliche Bildung notwendig sei (Suhadev et al. 2006).

Im Hinblick auf eine mögliche Vulnerabilität der Studienteilnehmerinnen der Fallstudie könnten die Ergebnisse der oben beschriebenen Studie zum einen aufzeigen, dass vor allem auch unter Frauen eine hohe Bereitschaft besteht, an einer HIV-Impfstudie teilzunehmen. Eine hohe Bereitschaft an einer HIV-Impfstudie teilzunehmen, könnte ein Anzeichen dafür sein, dass die Zustimmung zu einer Studie freiwillig erfolgte. Hinzu kommt, dass sich die verheirateten Frauen der Studie der potenziellen Gefahr bewusst waren, dass sie von ihrem Ehemann mit HIV infiziert werden könnten. Beide Aspekte könnten für eine selbstbestimmte Entscheidung für die Teilnahme einer HIV-Impfstudie sprechen, was möglicherweise auch auf die ProbandInnen der Fallstudie zutrifft.

Zum anderen zeigen die Ergebnisse der oben dargestellten Studie jedoch auch, dass viele Sorgen und Unsicherheiten mit der Teilnahme an einer HIV-Impfstudie verknüpft sind und dass eine große Hoffnung besteht, durch eine Studienteilnahme vor einer HIV-Infektion geschützt zu sein. Dies ist jedoch falsch, da die Wirksamkeit einer Impfung erst im Rahmen der Studie getestet wird und keinesfalls von einem Schutz vor HIV durch eine Studienteilnahme ausgegangen werden darf. Diese Problematik wird auch als therapeutic misconception bezeichnet. Lidz und Appelbaum (2002) definieren therapeutic misconception folgendermaßen: „A therapeutic misconception occurs when a research subject fails to appreciate the distinction between the imperatives of clinical research and of ordinary treatment, and therefore inaccurately attributes therapeutic intent to research procedures“ (Lidz and Appelbaum 2002, S. V57). Therapeutic misconception im Rahmen einer HIV-Impfstudie könnte zum einen die fatale Folge mit sich bringen, dass ProbandInnen während und nach der Studienteilnahme mehr Risikoverhalten zeigen. Zum anderen zeigt sich hierdurch möglicherweise mangelndes Verstehen von ProbandInnen bezüglich der Konzipierung einer HIV-Impfstudie.

Diese Gesichtspunkte und auch die zuvor dargestellten Zusatzinformationen zur Fallstudie sowie die erläuterten Kritikpunkte hinsichtlich ethischer Aspekte bei der Durchführung der Studie, sollen im folgenden Kapitel in die Überprüfung der potenziellen Vulnerabilität der Studienteilnehmerinnen miteinbezogen werden. 


\subsubsection{Vulnerabilität der StudienteilnehmerInnen in Fallstudie A}

In diesem Kapitel gilt es eine mögliche Vulnerabilität der in der Fallstudie A beteiligten StudienteilnehmerInnen normativ $\mathrm{zu}$ bewerten. Dies soll anhand der in Kapitel 3.4 aufgestellten Vulnerabilitätskriterien geschehen (siehe hierzu Abb. 1: Vulnerabilitätskriterien, S. 30). Die Frage, ob die StudienteilnehmerInnen im Kontext der analysierten Studie vulnerabel waren, wird anhand des Hauptvulnerabilitätskriteriums erböbte Wahrscheinlichkeit, Unrecht zu erfahren am Ende dieses Kapitels beantwortet. Inwiefern das Hauptvulnerabilitätskriterium zutrifft, soll wiederum anhand der Vulnerabilitätskriterien, die zu Kontext der Studie und zu Eigenschaften und Kontext der Person/Personengruppe zählen, geprüft werden.

An dieser Stelle soll klargestellt werden, dass die Überprüfung der Vulnerabilitätskriterien zwar alle ProbandInnen der Fallstudie miteinbezieht, dies allerdings nicht die Notwendigkeit einer Einzelfallbetrachtung im Sinne einer Prüfung der Vulnerabilität von einzelnen ProbandInnen ausschließst. Da keine detaillierten Informationen über die einzelnen ProbandInnen vorliegen, kann jedoch nicht aufgezeigt werden, ob einzelne ProbandInnen möglicherweise vulnerabel im Gegensatz zu anderen sind oder vulnerabler im Vergleich zu anderen ProbandInnen. Darüber hinaus muss daran erinnert werden, dass die Studie sowohl mit männlichen als auch weiblichen ProbandInnen durchgeführt wurde. Im Laufe der Interpretation sollen deswegen die Vulnerabilitätskriterien zunächst bezüglich aller ProbandInnen betrachtet werden und dann mit besonderem Fokus auf mögliche Unterschiede zwischen den männlichen und weiblichen ProbandInnen diskutiert werden. Außerdem ist es wichtig zu erwähnen, dass sich die Überprüfung der Vulnerabilität ausschließlich auf den Kontext der analysierten Studie bezieht und nicht im Allgemeinen auf andere Lebenslagen der ProbandInnen übertragbar ist.

\section{A: Kontext der Studie}

\section{Ethisch unzureichende Konzipierung der Studie}

Das erst genannte Vulnerabilitätskriterium ist die Ethisch un₹ureichende Konzipierung der Studie. Die analysierte Studie verfolgt gänzlich den Ansatz eine präventive Impfung gegen HIV zu entwickeln, was jedoch von Thomas (2004) und Jesani und Coutinho (2007b) kritisiert wird (Kapitel 4.2.1). Stattdessen geben sie (Thomas 2004; Jesani und Coutinho 2007b) Argumente für die vorrangige Entwicklung einer therapeutischen Impfung an. Im Rahmen dieser Arbeit ist es nicht möglich, endgültig zu klären, ob die Argumente für eine präventive oder für eine therapeutische Impfung schwerer aufwiegen. Aber es ist dennoch wichtig aufzuzeigen, dass eine präventive Impfung nicht die einzige und womöglich auch nicht die beste Lösung zur Kontrolle von HIV in Indien ist, wie von den AutorInnen der Fallstudie dargestellt.

Ein weiterer wichtiger Aspekt, der zur ethischen Bewertung der Studienkonzipierung zählt, ist die 
Beurteilung, ob Ausbeutung im Rahmen der Studienteilnahme stattgefunden hat. Dies wiederum ist abhängig davon, ob ein fairer Nutzen für die Studienteilnahme bereitgestellt wurde (siehe hierzu Kapitel 3.3). Um dies einschätzen zu können, gilt es zunächst, den potenziellen Nutzen einer Studienteilnahme darzustellen und diesen daraufhin mit den möglichen Risiken in Beziehung zu setzen, um das Nutzen-Risiko-Verhältnis bewerten zu können. Außerdem gilt es, für die Einschätzung eines fairen Nutzens in einem dritten Schritt den Nutzen für die ProbandInnen mit dem Nutzen für die SponsorInnen beziehungsweise für die Pharmafirma, die den Impfstoff entwickelte, zu vergleichen.

Nutzen für die ProbandInnen waren folgende:

- Krankenversicherung während der Studienlaufzeit

- Zusicherung einer angemessenen Therapie über fünf Jahre im Falle einer HIV-Infektion

- medizinische Behandlung und Kompensation für jegliche mit der Studie assoziierten gesundheitlichen Probleme

- Beratung hinsichtlich HIV-Tests, Verhütung und Verhalten zur HIV-Prävention

- Schutz vor einer HIV-Infektion, falls sich der Impfstoff als wirksam herausstellt

Der zuletzt genannte Aspekt kann allerdings im Falle der analysierte Studie nicht als Nutzen gewertet werden, da sich sowohl in Phase I- als auch in Phase II- Studien herausstellte, dass die Immunogenität des Impfstoffs nur mäßig ist und somit keine Wirkung im Sinne eines Schutzes vor einer HIV-Infektion zu erwarten ist.

Die Risiken und Nachteile einer Studienteilnahme waren folgende:

- Nebenwirkungen der Impfung

- riskantes Verhalten durch fälschliche Annahme, dass Impfung gegen HIV schützt

- Stigmatisierung, Diskriminierung und Verlust sozioökonomischer Sicherheiten im Falle des Bruchs der Vertraulichkeit bezüglich der Studienteilnahme

Letztere Gesichtspunkte beziehen sich auf soziale Risiken, die ProbandInnen mit einer Studienteilnahme eingehen und betreffen insbesondere Frauen und stellen somit für die weiblichen Studienteilnehmer im Vergleich zu den männlichen ein höheres Risiko dar. Allerdings wurde das Forscherteam mithilfe eines von Experten besetzten Gender Advisory Board bezüglich solcher Aspekte speziell geschult (Excler et al. 2008). Zudem wurde den ProbandInnen versichert, dass jegliche ihre Studienteilnahme betreffenden Informationen vertraulich behandelt werden (Mehendale et al. 2010). Die Nebenwirkungen und die Sicherheit des Impfstoffs betreffend ergaben die Studienergebnisse keine ernsthaften Bedenken und somit kann dieses Risiko als relativ gering eingeschätzt werden. Die Wahrscheinlichkeit, dass die Annahme, der Impfstoff schütze sicher gegen HIV, zu einem risikoreicheren Verhalten der ProbandInnen führe, scheint relativ gering, da ein Verständnistest über 
studienrelevante Informationen mit den ProbandInnen durchgeführt wurde. Zunächst scheint das Nutzen-Risiko-Verhältnis fair zu sein, da die meisten Risiken und Nachteile einer Studienteilnahme sich als ziemlich unwahrscheinlich oder unbegründet herausstellen. Die bisher genannten Aspekte beziehen sich allerdings gänzlich auf die Vor- und Nachteile, die die ProbandInnen direkt betreffen. Im Kontext transnationaler Biomedizin ist es jedoch auch wichtig, den Nutzen und die Risiken für die gesamte Bevölkerung des Gastlandes im Blick zu behalten. Zunächst muss hierbei beachtet werden, dass HIV und AIDS in Indien ein großes Problem darstellen und folglich Forschung, die zur Verminderung der Krankheitslast beitragen könnte, eine hohe lokale Relevanz aufweist. Der letztendliche Nutzen für die Bevölkerung Indiens scheint jedoch insofern gering, da sich der in der Fallstudie getestete Impfstoff als nicht wirksam herausstellte und somit keine Lösung für die Kontrolle von HIV in Indien gefunden wurde. Darüber hinaus gibt es, wie bereits erläutert, Zweifel, inwiefern der Ansatz einer präventiven Impfung für Indien sinnvoll ist, um die Krankheitslast von HIV zu senken, was wiederum die lokale Relevanz der Studie mindern könnte. Des Weiteren ist unklar, welche Vereinbarungen zwischen IAVI und der indischen Regierung über die Bereitstellung des Impfstoffs getroffen wurden für den Fall, dass sich dieser als wirksam herausstellen sollte. Die angemessene Bereitstellung eines als erfolgreich getesteten Medikaments nach Abschluss einer Studie gilt jedoch als eine mögliche Form eines fairen Nutzens. Insbesondere bei Forschung in Entwicklungsländern könnte hierdurch je nach Studienkontext Ausbeutung verhindert werden. ${ }^{10}$ Die Zweifel an einer solchen Bereitstellung des Impfstoffs führen zu der Annahme, dass kein fairer Nutzen für die indische Bevölkerung besteht, zu der auch die ProbandInnen zählen. Die Fairness des Nutzens im Kontext der Studie ist auch abhängig vom Nutzen für die ansonsten beteiligten Parteien. Hierbei lässt sich nur schwer bewerten, wie groß der Nutzen der Studiendurchführung für den Hauptsponsor IAVI ist. Da IAVI eine nicht auf Gewinn ausgerichtete Organisation ist, kann hier nicht von finanziell motivierten Studieninitiativen ausgegangen werden und demnach auch nicht von einem finanziellen Nutzen für IAVI. Den Nutzen für Targeted Genetics betreffend, lässt sich vermuten, dass sie, sowohl finanziell als auch das Prestige der Firma betreffend, davon profitiert hätten, wenn sich der von ihnen entwickelte und für die Studie bereitgestellte Impfstoff als wirksam zum Schutz gegen eine HIV-Infektion gezeigt hätte.

Hinsichtlich Ausbeutung im Kontext der Fallstudie lässt sich zusammenfassend festhalten, dass der Nutzen für die ProbandInnen selbst erstmal als fair einzuschätzen ist, da es keine substantiellen gegenteiligen Hinweise gibt. Die Fairness des Nutzens für die indische Bevölkerung lässt sich allerdings schwerer einordnen. Es wurden diesbezüglich Anhaltspunkte erläutert, die die lokale Relevanz der Forschung in Frage stellen. Zudem ist unklar, ob eine angemessene Bereitstellung der Impfung für Indiens Bevölkerung zugesichert wurde. Des Weiteren ist der Nutzen für den Hauptsponsor und für Targeted Genetics schwer einzuschätzen. Folglich kann anhand des Vulnerabilitätskriteriums Ethisch unzureichende Konzipierung der Studie nicht geklärt werden, ob die ProbandInnen im Kontext der Studie als

10 Siehe hierzu: World Medical Association (2008): Paragraph: 33; CIOMS (2002): Punkt: 10; UNESCO (2005): Artikel 15 
vulnerabel gelten sollten, da ein Impfstoff an ihnen getestet wurde, dessen lokale Relevanz umstritten ist und da der faire Nutzen für eine Studienteilnahme nicht abschließend geklärt werden kann.

\section{Ethische Ungültigkeit des informed consent}

Das zweite zum Kontext der Studie zugehörige Vulnerabilitätskriterium ist die Ethische Ungültigkeit des informed consent von Seiten des Forscherteams ausgehend. Betrachtet man diesbezüglich die Aufklärung der StudienteilnehmerInnen, scheint diese insofern unzureichend, dass dem Sponsor IAVI vor Beginn der Studie in Indien bereits Ergebnisse aus Europa vorlagen und die potenziellen ProbandInnen darüber nicht umgehend informiert wurden. Es ist aus ethischer Sicht problematisch, dass die StudienteilnehmerInnen diesbezüglich nicht ausreichend aufgeklärt wurden und somit ihre Entscheidung für oder wider eine Studienteilnahme unter falschen Annahmen trafen. Die Deklaration von Helsinki fordert in Paragraph 26, dass ProbandInnen unter anderem über alle relevanten Aspekte der Studie informiert werden müssten (WMA 2013). Die Universal Declaration on Bioethics and Human Rights der UNESCO (Artikel 6) erläutert hinsichtlich der Aufklärung für eine Studienteilnahme, dass diese angemessen und in verständlicher Form sein soll (UNESCO 2005). ProbandInnen über Ergebnisse aus vorherigen Studien zu informieren sollte unter relevante Aspekte beziehungsweise angemessene Aufklärung fallen. Inwiefern anhand der oben genannten Richtlinien der informed consentProzess von Seiten des Forscherteams ethisch unzureichend ist, bleibt jedoch diskutabel, da Begriffe wie relevant und angemessen unterschiedlich interpretiert werden könnten. Die International Ethical Guidelines for Biomedical Research Involving Human Subjects der CIOMS erläutern ihre Forderungen für einen ethisch hinreichenden informed consent jedoch wesentlich detaillierter. In Punkt 6 verpflichten sie SponsorInnen und ForscherInnen den informed consent aller ProbandInnen zu erneuern, falls sich bedeutende Veränderungen bezüglich der Studienbedingungen ergeben (CIOMS 2002). Die Forderungen der Richtlinien und die oben genannten Aspekte mitberücksichtigend gilt das Vulnerabilitätskriterium Ethische Ungültigkeit des informed consent, das zum Kontext der Studie zählt, zumindest teilweise als zutreffend. An dieser Stelle muss allerdings auch erwähnt werden, dass im Rahmen des informed consent-Prozesses viele Maßnahmen, wie beispielsweise der Verständnistest, angewendet wurden, um eine ethische Ungültigkeit des informed consent zu verhindern. Zusätzlich wurde der informed consent-Prozess ausführlich beschrieben, was als positiv zu bewerten gilt, da hierdurch Transparenz hinsichtlich ethischer Aspekte geschaffen wird. Beide Vulnerabilitätskriterien, die zum Kontext der Studie zählen, zeigen die analysierte Studie betreffend keine Unterschiede zwischen den männlichen und den weiblichen Studienteilnehmern auf. 


\section{B: Eigenschaften und Kontext der Person/Personengruppe}

\section{Prädisposition, zusätzliches Leid zu erfabren}

Nun gilt es die Vulnerabilitätskriterien zu prüfen, die die Eigenschaften und Kontext der Person/Personengruppe betreffen und folglich von den ProbandInnen selbst oder durch deren Lebenssituation zu Vulnerabilität führen könnten. Das erste Vulnerabilitätskriterium hierzu ist die Prädisposition, zusätəliches Leid zu erfahren. Für die Erfüllung dieses Vulnerabilitätskriteriums gibt es im Kontext der analysierten Studie keine Anhaltspunkte, da keine Informationen vorliegen, ob die StudienteilnehmerInnen vorheriges Leid erfahren haben und es auch keine Hinweise auf zusätzliches Leid im Rahmen der Studienteilnahme gab. Bezieht man die in Kapitel 4.2.2 aufgeführten Informationen hinsichtlich einer anhaltenden Diskriminierung vieler Frauen in Indien in Betracht, erscheint es jedoch logisch, dass die Anfälligkeit für zusätzliches Leid mit erhöhter Wahrscheinlichkeit die weiblichen Probanden betrifft. Da es hierzu allerdings keine genaueren Informationen bezüglich der ProbandInnen der analysierten Fallstudie gibt, bleibt es dabei, dass dieses Vulnerabilitätskriterium nicht zutrifft.

\section{Unfähigkeit, informed consent zu erteilen und/oder Anfälligkeit, unfreiwillig quzustimmen}

Das zweite Vulnerabilitätskriterium, das es zu prüfen gilt und zu Eigenschaften und Kontext der Person/Personengruppe zählt, ist die Unfäbigkeit, informed consent zu erteilen und/oder Anfälligkeit, unfreiwillig zuqustimmen. Hier gilt es den informed consent-Prozess von Seiten der ProbandInnen in den Fokus zu rücken. Da die ProbandInnen vor der eigentlichen Einwilligung einen Verständnistest zu studienrelevanten Informationen bestehen mussten, kann davon ausgegangen werden, dass das Verstehen als eines der Elemente eines ethisch gültigen informed consent gegeben ist. Hinsichtlich der Freiwilligkeit der ProbandInnen gibt es keine Hinweise auf eine Ausübung von Zwang oder Manipulation. Darüber hinaus wurde die Höhe der Aufwandsentschädigung für alle ProbandInnen standardisiert festgelegt, um zu verhindern, dass diese durch einen unangemessenen Anreiz beeinflusst werden (Jesani und Coutinho 2007b). Um letzteres auszuschließen müsste allerdings auch geklärt werden, wie hoch die Aufwandsentschädigung war und wie diese im Verhältnis zur sozioökonomischen Situation der ProbandInnen einzuordnen ist. Diese Informationen liegen allerdings nicht vor. Im Kontext des informed consent ist es wichtig zu erwähnen, dass es hinsichtlich des Bildungsstatus Unterschiede zwischen den weiblichen und männlichen Probanden gab und hierbei die Frauen im Durchschnitt einen niedrigeren Bildungsstatus hatten. Dies deckt sich auch weitestgehend mit den in Kapitel 4.2.2 dargestellten Informationen über Alphabetisierung und Bildungsstand von Frauen in Indien im Vergleich zu Männern. Falls im Rahmen der Studie folglich ProbandInnen aufgrund eines niedrigeren Bildungsstandes eventuell unfähig waren einen ethisch gültigen informed consent zu erteilen, könnte dies die weiblichen Probanden mit einer höheren Wahrscheinlichkeit betreffen als die männlichen. An dieser Stelle muss zudem auf die in Kapitel 5.2.1 erläuterte Studie zur Bereitschaft an 
einer HIV-Impfstudie teilzunehmen, erinnert werden. Diese liefert auf der einen Seite Hinweise auf eine hohe Wahrscheinlichkeit, dass potenzielle ProbandInnen in HIV-Impfstudien freiwillig zustimmen und eine selbstbestimmte Entscheidung für oder wider eine Studienteilnahme treffen. Es gibt jedoch auch Hinweise darauf, dass das Wissen über HIV-Impfstudien sehr gering ist, was eine selbstbestimmte Entscheidung auf der anderen Seite unwahrscheinlicher werden lässt. Zudem zeigt diese Studie, dass die Hoffnung durch die Teilnahme an einer HIV-Impfstudie vor einer HIV-Infektion geschützt zu werden, vermutlich sehr groß ist (Suhadev et al. 2006). Hierdurch könnte die Entscheidung für eine Studienteilnahme beeinflusst werden, obwohl die Teilnahme an einer HIV-Impfstudie keinen Schutz vor einer Infektion bedeutet, da während der Studie noch nicht sicher ist, ob und wie wirksam der getestete Impfstoff sein wird. Demnach würden die potenziellen ProbandInnen ihre Entscheidung auf eine Hoffnung gründen, die vermutlich nicht erfüllt werden wird, da der Versuch einen wirksamen HIV-Impfstoff zu finden bisher gescheitert ist. Es muss jedoch darauf hingewiesen werden, dass die beschriebene Studie mit einer relativ niedrigen Teilnehmeranzahl (112) durchgeführt wurde, was die Repräsentativität der Ergebnisse mindert und zudem können die Antworten und Einstellungen nicht unmittelbar auf die ProbandInnen der Fallstudie übertragen werden. Insgesamt betrachtet gibt es nämlich im Kontext der Fallstudie keine Anhaltspunkte für die Studienteilnahme von ProbandInnen, weder weibliche noch männliche, die unfähig waren, einen informed consent zu erteilen oder anfällig dafür waren unfreiwillig zuzustimmen. Demnach gilt dieses Vulnerabilitätskriterium als nicht zutreffend. Hierfür sprechen zudem die zusätzlichen Vorsichts- und Schutzmaßnahmen, wie beispielsweise der zweistufige informed consent-Prozess und der Verständnistest, die sowohl das Verstehen als auch die Freiwilligkeit im Rahmen des informed consent sicherstellen sollen.

\section{Anfälligkeit, ausgebentet zu werden}

Die Anfälligkeit, ausgebeutet zu werden als drittes Vulnerabilitätskriterium, das zu Eigenschaften und Kontext der Person/Personengruppe zählt, ist abhängig davon, ob die ProbandInnen aufgrund ihrer Lebenssituation eine erhöhte Wahrscheinlichkeit aufweisen, einem unfairen Nutzen zuzustimmen. Inwiefern ein fairer Nutzen für die Studienteilnahme bereitgestellt wurde, wurde bereits anhand des Vulnerabilitätskriteriums Ethisch unzureichende Kon₹ipierung der Studie diskutiert, konnte jedoch nicht abschließend geklärt werden. Aspekte, die die Lebenssituation von ProbandInnen betreffen und die Wahrscheinlichkeit erhöhen, dass diese einem unfairen Nutzen zustimmen würden, sind beispielsweise die sozioökonomische Situation und der Bildungsstand. Diesbezüglich lässt sich festhalten, dass alle ProbandInnen der Fallstudie mindestens einen Schulabschluss bis zum Ende der zehnten Klasse absolvierten. Zusätzlich waren alle ProbandInnen berufstätig. Demnach ist bei der Fallstudie nicht davon auszugehen, dass sich die ProbandInnen zum Zeitpunkt der Studienteilnahme in einer prekären sozioökonomischen Situation befanden. Weitere Aspekte, die eine Anfälligkeit, ausgebeutet zu werden, bedingen könnten, wie zum Beispiel Machtlosigkeit, können anhand der gegebenen Informationen zur 
Lebenssituation der ProbandInnen nicht überprüft werden. Zudem muss erwähnt werden, dass die Aspekte, die dieses Vulnerabilitätskriterium betreffen, Unterschiede zwischen den männlichen und weiblichen Studienteilnehmern aufweisen. Dies zeigt sich anhand der unterschiedlichen Verteilung des Schulabschlusses zwischen männlichen und weiblichen Probanden. Insgesamt lässt dies jedoch nicht den Schluss zu, dass die weiblichen Probanden im Gegensatz zu den männlichen anfällig für Ausbeutung waren. Es wird allerdings deutlich, dass es bei der Bewertung einer möglichen Vulnerabilität von ProbandInnen im Kontext transnationaler Biomedizin nicht unerheblich ist, ob Männer oder Frauen an medizinischen Studien teilnehmen. Abschließend lässt sich nicht endgültig klären, ob das Vulnerabilitätskriterium Anfälligkeit, ausgebentet zu werden zutrifft. Die Wahrscheinlichkeit ist jedoch aufgrund der gegebenen Informationen über die Lebensbedingungen der ProbandInnen eher gering.

Nach der Überprüfung der Vulnerabilitätskriterien, die zu Kontext der Studie und zu Eigenschaften und Kontext der Person/Personengruppe zählen, zeigt sich, dass das Hauptvulnerabilitätskriterium Erhöhte Wabrscheinlichkeit, Unrecht zu erfahren zutrifft und die ProbandInnen im Rahmen ihrer Studienteilnahme teilweise als vulnerabel gelten können. Zum einen ist dies durch die Erfüllung des Vulnerabilitätskriteriums Ethische Ungültigkeit des informed consent bedingt, das die ungenügende Aufklärung der ProbandInnen von Seiten des Forscherteams im Hinblick auf die Studienergebnisse aus Europa betrifft. Zum anderen ist es nicht unerheblich, dass die Erfüllung des Vulnerabilitätskriteriums Ethisch unzureichende Kon₹ipierung der Studie nicht sicher zu klären ist. Da ein Vulnerabilitätskriterium zutrifft und ein zweites möglicherweise zutrifft, wird deutlich, dass Vulnerabilität verschiedene Grade aufweisen kann. Hierbei ist entscheidend, wie viele Vulnerabilitätskriterien zutreffend sind, wie schwerwiegend die Gründe für das Zutreffen dieser Vulnerabilitätskriterien sind oder inwiefern das Zutreffen von Vulnerabilitätskriterien nicht ausgeschlossen werden kann. Würde man mögliche Schutzmaßnahmen für vulnerable Personen oder Personengruppen eruieren, müsste man Aspekte, die nur möglicherweise, nicht aber sicher ein Vulnerabilitätskriterium erfüllen, selbstverständlich mit berücksichtigen. Im Hinblick auf die Vulnerabilität der ProbandInnen der analysierten Studie muss zusätzlich erwähnt werden, dass von den ForscherInnen viele Maßnahmen angewendet wurden, um sowohl bei der Vorbereitung als auch bei der Durchführung der Studie eine mögliche Vulnerabilität der Studienteilnehmerinnen zu verhindern. Hierzu zählen zum Beispiel das Community Advisory Board und das Gender Advisory Board sowie der Verständnistest im Rahmen der Einwilligung. Des Weiteren fällt auf, dass einige Zusatzinformationen zu den ProbandInnen und deren Lebenssituation veröffentlicht wurden. Dies zeigt, dass für die ForscherInnen Informationen, wie beispielsweise die sozioökonomische Situation der StudienteilnehmerInnen, von Bedeutung sind und sie möglicherweise davon ausgingen mit einer vulnerablen Gruppe zu forschen. Mit Blick auf die umfassenden Vorbereitungen auf die Studie, die Vorsichtsmaßnahmen während des informed consent-Prozesses und die zusätzlich zur 
Verfügung gestellten Informationen über die ProbandInnen wird deutlich, dass das Konzept der Vulnerabilität hilfreich sein kann, um solche Maßnahmen zu fordern. Durch die Schutzmaßnahmen und Vorbereitungen, die hohe ethische Standards für HIV-Studien in Indien schaffen sollten, entsteht der Eindruck, dass die ProbandInnen im Kontext der Studie nicht vulnerabel waren. Dieser Eindruck täuscht jedoch, da die Aufklärung unzureichend abgelaufen ist und somit die ProbandInnen ihre Entscheidung für oder gegen eine Studienteilnahme unter falschen Annahmen trafen. Es wäre nämlich möglich, dass sich ProbandInnen aufgrund der zusätzlichen Informationen einerseits gegen eine Studienteilnahme entschieden hätten, da sie davon ausgegangen wären, es gäbe bereits Forschungsergebnisse zu diesem Impfstoff und somit müsste dieser nicht erneut an ihnen getestet werden. Andererseits könnte es auch sein, dass sich potenzielle ProbandInnen eher für eine Studienteilnahme entschieden hätten, da sie aufgrund der Ergebnisse aus Europa weniger Befürchtungen hinsichtlich schwerwiegender Nebenwirkungen gehabt hätten. Bisher konnte nur geklärt werden, dass die ProbandInnen im Rahmen der Studie vulnerabel waren. Inwiefern sich jedoch die Vulnerabilität der weiblichen von der der männlichen Studienteilnehmer unterscheidet, gilt es nun genauer zu untersuchen. Hierzu ist es wichtig, sich den durchschnittlich niedrigeren Bildungsstatus der Probandinnen im Vergleich zu den Probanden in Erinnerung zu rufen. Demnach zeigt sich eine erhöhte Wahrscheinlichkeit, dass die weiblichen Probanden im Vergleich zu den männlichen vulnerabler sein könnten, obgleich das zutreffende Vulnerabilitätskriterium Ethische Ungültigkeit des informed consent für diese Studie auf alle ProbandInnen gleichermaßen zutrifft und es hierbei keine Aspekte für diesbezügliche Unterschiede zu nennen gibt. Die Tatsache, dass für die Vorbereitung der Studie ein sogenanntes Gender Advisory Board gegründet wurde und die ForscherInnen hinsichtlich geschlechtsspezifischer Aspekte geschult wurden, gibt einen Hinweis darauf, dass die Forschung mit Frauen in Indien andere ethische Standards erfordert als die Forschung mit Männern. Inwiefern dies indirekt andeutet, dass Frauen in Indien im Kontext transnationaler Biomedizin eine erhöhte Wahrscheinlichkeit aufweisen vulnerabel zu sein, ist anzunehmen, kann jedoch nicht mit Sicherheit geklärt werden. Zudem wurde darauf geachtet, dass Frauen und Männer in einem ähnlichen Verhältnis rekrutiert werden. Im Nachhinein wurde dies aus ethischer Sicht positiv bewertet, da Frauen in Indien selten an Studien teilnehmen würden (Excler et al. 2008). Dies zeigt wiederum, dass das Forscherteam der analysierten Beispielstudie Aspekte, die bei Forschung mit Männern in Indien beachtet werden sollten, nicht mit Aspekten gleichsetzt, die Forschung mit Frauen in Indien betreffen. Dies stützt die These, dass bei der ethischen Bewertung einer möglichen Vulnerabilität von ProbandInnen im Kontext transnationaler Biomedizin nicht nur die Tatsache von Bedeutung ist, dass ForscherInnen aus Industriestaaten in Entwicklungsländern Studien durchführen, sondern auch ob Frauen oder Männer an der Studie teilnehmen.

Des Weiteren lässt sich anhand der analysierten Studie beispielhaft ein Problem im Kontext transnationaler Biomedizin darlegen, nämlich Kommunikationsschwierigkeiten zwischen SponsorInnen in 
Industriestaaten und ForscherInnen in Entwicklungsländern. In der Fallstudie ist dies sogar mit ursächlich für die Vulnerabilität der ProbandInnen, da IAVI zuerst die Pharmafirma über die Ergebnisse aus Europa informierte und erst daraufhin das Forscherteam in Indien. Dies führte wiederum dazu, dass die ProbandInnen unzureichend aufgeklärt wurden. Um die hohen und auch notwendigen ethischen Standards, die IAVI für die Studie in Indien aufstellen wollte tatsächlich zu erfüllen, hätten diese ihre Priorität bei der Zusammenarbeit mit dem indischen Forscherteam setzen müssen, anstatt bei der Pharmafirma, die nicht einmal Co-Sponsor war. Hiermit hätte IAVI zeigen können, dass ihre oberste Priorität den ProbandInnen gilt.

Als Gesamtüberblick wird die Überprüfung der Vulnerabilitätskriterien für die Fallstudie A in der nachfolgenden Tabelle nochmal zusammenfassend dargestellt. 


\begin{tabular}{|c|c|c|c|c|}
\hline & & \multicolumn{3}{|c|}{ Fallstudie A: HIV-Impfstudie } \\
\hline \multirow{6}{*}{ 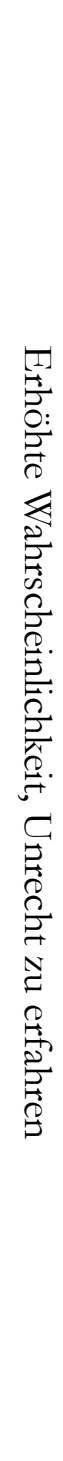 } & & Vulnerabilitätskriterien & Zutreffend? & $\begin{array}{l}\text { zugrundeliegende Aspekte für das (Nicht)- } \\
\text { Zutreffen des Vulnerabilitätskriteriums }\end{array}$ \\
\hline & \multirow[t]{2}{*}{ 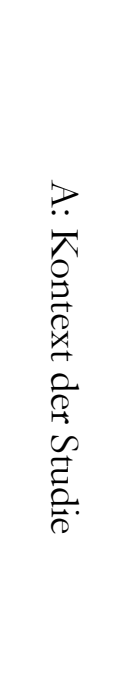 } & $\begin{array}{l}\text { I. Ethisch unzureichende } \\
\text { Konzipierung der Studie }\end{array}$ & $?$ & $\begin{array}{l}\text { - lokale Relevanz fragwürdig, da nur } \\
\text { präventive Impfung erforscht und } \\
\text { therapeutische Impfung evtl. für Indien } \\
\text { sinnvollere Lösung } \\
\text { - Bedingungen für Bereitstellung der } \\
\text { Impfung nach Abschluss der Studie } \\
\text { nicht veröffentlicht } \\
\text { - } \quad \text { Nutzen für ProbandInnen fair } \\
\text { - Fairness des Nutzens für die } \\
\text { Gesamtbevölkerung kann nicht } \\
\text { abschließend geklärt werden }\end{array}$ \\
\hline & & $\begin{array}{l}\text { II. Ethische Ungültigkeit } \\
\text { des informed consent }\end{array}$ & ja & $\begin{array}{l}\text { - Aufklärung unzureichend, da } \\
\text { ProbandInnen nicht unverzüglich über } \\
\text { Studienergebnisse aus Europa } \\
\text { informiert wurden }\end{array}$ \\
\hline & \multirow{3}{*}{ 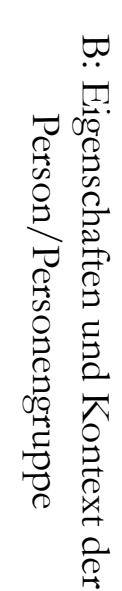 } & $\begin{array}{l}\text { I. Prädisposition, } \\
\text { zusätzliches Leid zu } \\
\text { erfahren }\end{array}$ & nein & $\begin{array}{l}\text { - keine Anhaltspunkte für vorheriges } \\
\text { Leid oder Leid während der } \\
\text { Studienteilnahme }\end{array}$ \\
\hline & & $\begin{array}{l}\text { II. Unfähigkeit, informed } \\
\text { consent zu erteilen und/oder } \\
\text { Anfälligkeit, unfreiwillig } \\
\text { zuzustimmen }\end{array}$ & nein & $\begin{array}{l}\text { - Schutzmaßnahmen im Rahmen des } \\
\text { informed consent-Prozesses (z.B. } \\
\text { Verständnistest usw.) }\end{array}$ \\
\hline & & $\begin{array}{l}\text { III. Anfälligkeit, } \\
\text { ausgebeutet zu werden }\end{array}$ & nein & $\begin{array}{l}\text { - Informationen über } \\
\text { Lebensbedingungen machen } \\
\text { Anfälligkeit für Ausbeutung } \\
\text { unwahrscheinlich }\end{array}$ \\
\hline
\end{tabular}




\subsection{Fallstudie B: 'Evaluation of the Immune and Safety Response of GlaxoSmithKline (GSK) Biologicals' HPV Vaccine in Healthy Indian Women'}

In diesem Kapitel folgt nun die Analyse der zweiten Fallstudie, die mit Frauen in Indien durchgeführt wurde. Die deskriptive Analyse konzentriert sich neben allgemeinen Informationen zur Studie auf Faktoren, die eine mögliche Vulnerabilität der Studienteilnehmerinnen bedingen könnten. Die diesbezügliche normative Bewertung wird in Kapitel 5.4 folgen. Die zu analysierende Studie wurde im Juni 2006 mit dem Titel Evaluation of the Immune and Safety Response of GlaxoSmithKline (GSK) Biologicals' HPV Vaccine in Healtby Indian Women bei clinicaltrials.gov registriert und im Dezember 2007 abgeschlossen. Das Ziel der Studie war es, die Immunogenität und die Sicherheit der HPV-16/18 AS04Adjuvans-Impfung (Cervarix) für erwachsene Frauen (18 bis 35 Jahren) in Indien zu testen (Bhatla et al. 2010, S. 123).

Folgende Publikation wurde zur Fallstudie veröffentlicht und dient somit als Informationsgrundlage für die Analyse:

- Bhatla N, Suri V, Basu P, Shastri S, Datta SK, Bi D, Descamps DJ, Bock HL (2010): Immunogenicity and safety of human papillomavirus-16/18 AS04-adjuvanted cervical cancer vaccine in healthy Indian women. Journal of Obstetrics and Gynaecology Research $\underline{36}$, 123-132

Das Abstract der Publikation lautet:

„Aim: India has the highest number of annual incident cases and mortality rates for cervical cancer worldwide. This study was conducted to assess the immunogenicity and safety of human papillomavirus (HPV)-16/18 AS04-adjuvanted cervical cancer vaccine in healthy Indian women aged 18-35 years old.

Methods: This double-blind, randomized (1:1), controlled and multicenter trial with two parallel groups, the Vaccine and Placebo groups, included 354 subjects in four centers across India. Subjects were given Glaxo-SmithKline's HPV-16/18 AS04-adjuvanted cervical cancer vaccine or aluminum hydroxide placebo according to a 0,1 and 6 month schedule and followed up until month 7. Serum samples were drawn at pre-vaccination and at month 7 . Safety data were collected throughout the study.

Results: A total of 330 subjects completed the study. One month post-Dose 3, all initially seronegative subjects in the Vaccine group had seroconverted for HPV-16 and HPV-18 antibodies with anti-HPV-16 and anti-HPV-18 geometric mean titer levels of 10226.5 EL.U/ml $(95 \%$ confidence interval: 8847.1$11821.0)$ and 3953.0 EL.U/ml (95\% confidence interval: 3421.8-4566.8), respectively. Initially seropositive subjects also showed an increase to similar geometric mean titer levels. Six serious adverse events (two in the Vaccine group and four in the Placebo group), all unrelated to vaccination, were reported. Commonly reported solicited local (injection-site pain) and general (fatigue, headache and fever) symptoms were similar in both groups. Compliance to the three-dose vaccination course was $>97 \%$.

Conclusions: The AS04-adjuvanted HPV-16/18 cervical cancer vaccine was highly immunogenic and generally well-tolerated making it a potential tool for prevention and control of cervical cancer in India" (Bhatla et al. 2010, S. 123). 


\subsubsection{Hintergrundinformationen und aktueller Forschungsstand zu Fallstudie B}

Der folgende Abschnitt bietet zunächst Hintergrundinformationen zu Gebärmutterhalskrebs und den humanen Papillomviren (HPV) sowie zu HPV-Impfungen. Gebärmutterhalskrebs (Zervixkarzinom) ist weltweit betrachtet der dritthäufigste bösartige Tumor bei Frauen (Ferlay et al. 2010, S. 2902). Es ist wissenschaftlich belegt, dass Zervixkarzinome als Folge einer HPV-Infektion entstehen. Es gibt mehrere Typen von HPV, von denen 15 als Hochrisiko-HPV-Typen hinsichtlich der Entstehung von Zervixkarzinomen gelten. Eine HPV-Infektion wird über sexuellen Kontakt übertragen (STIKO: Ständige Impfkommission am Robert Koch-Institut 2009, S. 319). Die meisten HPV-Infektionen werden vom Immunsystem abgewehrt, führen jedoch nicht zu einer Immunität, das heißt, eine erneute Infektion mit demselben HPV-Typ ist möglich (STIKO 2009, S. 319). Zu einem geringen Teil werden HPV-Infektionen nicht vom Immunsystem überwunden und bleiben bestehen (circa 10\%). Verlaufen diese chronischen Infektionen über ein bis zwei Jahre, stellen sie ein sehr hohes Risiko für die Entstehung von Vorstufen von Zervixkarzinomen dar (Schiffmann et al. 2007, S. 893). Die Zellveränderungen werden in verschiedene Schweregrade eingeteilt (CIN: Cervical Intraepithelial Neoplasia 1-3). CIN 1 bedeutet nur geringgradige, CIN 2 mäßiggradige und CIN 3 hochgradige Zellveränderung oder ein Carcinoma in situ (Arzneitelegramm 2007). ${ }^{11}$ Schweregrade ab CIN 2 und höher, auch CIN 2+ genannt, gelten als Krebsvorstufen. Je höher der Schweregrad (CIN), desto höher ist das Risiko für die Entstehung von Zervixkarzinomen (STIKO 2009, S. 320). Das Risiko bei einem Schweregrad von CIN 3 innerhalb von zwei Jahren ein Zervixkarzinom zu entwickeln, liegt bei circa 50\% (STIKO 2009, S. 320). Deswegen wird empfohlen CIN 3 und über ein Jahr bestehende CIN 2 operativ zu behandeln (STIKO 2009, S. 320). 70\% der Zervixkarzinome werden durch HPV-Typ 16 oder 18 verursacht (STIKO 2009, S. 320; Gerhardus 2009). Im Jahre 2008 erkrankten in Deutschland circa 4.900 Frauen an einem Zervixkarzinom und 1.596 Frauen verstarben daran (Robert Koch Institut und Gesellschaft der epidemiologischen Krebsregister in Deutschland e. V. 2012). Insgesamt betrachtet ist das Zervixkarzinom in Deutschland aber eine verhältnismäßig seltene Erkrankung und die Inzidenz ist zudem in den letzten Jahrzehnten gesunken (Gerhardus 2009). Hochrechnungen zufolge muss jedoch etwa jede fünfte Frau in Deutschland im Laufe ihres Lebens einen operativen Eingriff zur Abklärung oder Behandlung von Krebsvorstufen am Gebärmutterhals durchführen lassen (STIKO 2009, S. 320). In Entwicklungsländern tritt das Zervixkarzinom wesentlich häufiger auf. Hier steht das Zervixkarzinom mit etwa 452.000 Neuerkrankungen im Jahre 2008 nach Brustkrebs an zweiter Stelle der am häufigsten vorkommenden Krebserkrankungen bei Frauen (Ferlay et al. 2010, S. 2902). Eine hohe Inzidenz und Mortalität von Zervixkarzinomen stellt vor allem auch für Frauen in Indien ein großes Problem dar. Hussain schreibt hierzu: „Cancer of the uterine cervix is a major cancer and a

11 Carcinoma in situ (CIS): Frühstadium eines Tumors, welcher die Basallamina noch nicht durchbrochen hat und somit nicht invasiv wächst und nicht metastasiert. 
leading cause of cancer-related deaths in women in India" (Hussain et al. 2012, S. 298).

Mit dem Ziel, Zervixkarzinomen vorzubeugen, gibt es schon seit einigen Jahren HPV-Impfungen. Die Firma Merck\&Co entwickelte den Impfstoff Gardasil gegen die HPV-Typen 16, 18, 6 und 11. Die beiden letzten HPV-Typen sind vor allem für Genitalwarzen verantwortlich. Gardasil wurde in zwei großen Studien FUTURE I und II (Females United to Unilaterally Reduce Endo/Ectocervical Disease) getestet und 2006 in Deutschland zugelassen (STIKO 2009, S. 321). Im Jahre 2007 wurde zusätzlich Cervarix zugelassen, ein von GlaxoSmithKline (GSK) Biologicals entwickelter Impfstoff gegen HPVTyp 16 und 18 (STIKO 2009, S. 323). Getestet wurde Cervarix in der sogenannten PATRICIA-Studie (PApilloma TRIal against Cancer In young Adults) und ist der HPV-Impfstoff, der auch in der zu analysierenden Fallstudie verwendet wurde (STIKO 2009, S. 323; Bhatla et al. 2010). Beide Impfstoffe enthalten Partikel, die bestimmten Proteinen der HP-Viruskapsel ähneln. Durch diese Partikel wird das Immunsystem angeregt spezifische Antikörper gegen das jeweilige HPV zu bilden, wodurch sich der Wirkmechanismus der Impfstoffe erklärt. Die Impfstoffe enthalten keine Virus-DNA und sind dadurch nicht infektiös (Arzneitelegramm 2007, S. 101). Um die Immunantwort zu verstärken, enthält Cervarix im Gegensatz zu Gardasil ein sogenanntes Adjuvans (Hilfsstoff) namens AS04, bestehend aus Monophosphoryl-Lipid A gebunden an Aluminiumhydroxid (Arzneitelegramm 2007, S. 101). Die STIKO sprach im März 2007 eine Empfehlung der HPV-Impfung für Mädchen zwischen 12 und 17 Jahren aus. Sie begründete dies durch die Reduktion der Krankheitslast von Zervixkarzinomen mithilfe der Impfung (STIKO 2007). In kritischer Reaktion auf diese Empfehlung forderten 2008 mehrere deutsche WissenschaftlerInnen eine Neubewertung der HPV-Impfung (Dören et al. 2008). Daraufhin veröffentlichte die STIKO 2009 eine überarbeitete Fassung der Begründung, wobei es allerdings bis heute bei der Impfempfehlung geblieben ist (STIKO 2012, S. 316). In den durchgeführten Studien zu den HPV-Impfstoffen (FUTURE I und II, PATRICIA) wird die Wirksamkeit der Impfung als ein selteneres Auftreten von Zervixkarzinomvorstufen in der Impfgruppe im Vergleich zur Placebogruppe definiert. Bei der FUTURE I- und II-Studie, die randomisiert, doppelt blind und Placebo-kontrolliert durchgeführt wurde, nahmen insgesamt mehr als 15.000 Probandinnen im Alter von 15 bis 26 Jahren teil (STIKO 2009, S. 321). In der Studiengruppe, die vor der Impfung bezüglich HPV-Typ 16 und/oder 18 als negativ getestet wurde und alle drei vorgeschriebenen Impfungen erhielt, zeigte sich eine Wirksamkeit zur Verhinderung von CIN2+ hinsichtlich HPV-Typ 16 und 18 von 97-100\%. Bei der Gruppe der Studienteilnehmerinnen, die vor der Impfung einen negativen HPV-Status bezüglich Typ 16, 18, 6, 11 und zehn weiteren onkogenen (krebserzeugenden) HPV-Typen hatten und somit die eigentliche Zielgruppe, nämlich Mädchen und junge Frauen vor dem ersten Geschlechtsverkehr, darstellt, zeigte sich eine Wirksamkeit zur Verhinderung von CIN 2+ unabhängig vom HPV-Typ, also nicht nur 16, 18, 6 und 11 betreffend, von 46\% (STIKO 2009, S. 323). Das bedeutet, das gesamt betrachtete Risiko, durch HPV-Typ 16 und 18 aber auch bezüglich anderer onkogener HPV-Typen Zervixkarzinomvorstufen zu entwickeln, wird durch Gardasil verringert. Allerdings belegten die 
Studien, dass die Wirksamkeit der Impfung bei schon bestehender HPV-Infektion wesentlich geringer ist. Dies führte zur Empfehlung Mädchen vor dem ersten Geschlechtsverkehr impfen zu lassen. Hieraus ergibt sich die Altersempfehlung von 12 bis 17 Jahren (STIKO 2009, S. 323ff). In der PATRICIA-Studie, einer Phase III-Studie zur Testung von Cervarix (Impfstoff gegen HPV-Typ 16 und 18), nahmen mehr als 18.000 Probandinnen im Alter zwischen 15 und 25 Jahren teil (Paavonen et al. 2009, S. 302ff). Diese Studie wurde 2009 im renomierten Journal Lancet abschließend publiziert (Paavonen et al. 2009). In die Studie einbezogen wurden Frauen, die bis zum Zeitpunkt der Studie nicht mehr als sechs verschiedene Geschlechtspartner angaben. Sie stimmten zu während der Studienlaufzeit adäquaten Konzeptionsschutz zu verwenden. Zudem durften sie keine Verändeurngen an der Zervix aufweisen. Ein negativer HPV-Status galt jedoch nicht als Einschlusskriterium, was bedeutet, dass auch Probandinnen an der Studie teilnehmen durften, die bereits HPV-infiziert sind (Paavonen et al. 2009, S. 304). Die PATRICIA-Studie wurde randomisiert, doppelt blind und Placebo-kontrolliert durchgeführt. Die Studienteilnehmerinnen wurden in verschiedene Gruppen eingeteilt. Die Impfgruppe erhielt zum Zeitpunkt 0, nach einem und sechs Monaten die HPV-16/18 AS04-Adjuvans-Impfung (Cervarix) und die Kontrollgruppe erhielt als Placebo eine Hepatitis-A-Impfung. Das Hauptziel der Studie war die Wirksamkeit von Cervarix gegen HPV 16 oder 18 assoziierte Zervixkarzinomvorstufen (CIN 2+) für Frauen mit einem zuvor negativen HPV-Status zu testen (Paavonen et al. 2009, S. 302). Für die Auswertung der Ergebnisse wurden verschiedene Gruppen anhand des HPV-Status (DNA der onkogenen HPV-Typen), Serostatus (Antikörper im Blut gegen HPV 16 und 18), Anzahl der erhaltenen Impfdosen und anhand der Befunde eines zytologischen Abstrichs (Vorliegen von Zellveränderungen) definiert (Paavonen et al. 2009, S. 302ff). Bei der Gruppe, die als HPV-naiv galt, also einen negativen HPV-Status bezüglich 16, 18 und zwölf weiteren onkogenen HPV-Typen und einen negativen Serostatus bezüglich HPV 16 und 18 aufwiesen, zeigte sich eine Wirksamkeit zur Verhinderung von HPV 16 und 18 assoziierten CIN 2+ von 98\%. Die Wirksamkeit zur Verhinderung von CIN 2+ unabhängig vom verursachenden HPV-Typ, also auch andere HPV-Typen als 16 und 18 betreffend, ergab in dieser Gruppe 70\% (Paavonen et al. 2009, S. 311). Für die Studiengruppe, die weder einen negativen HPV-Status, Serostatus noch einen unauffälligen zytologischen Befund aufwies, also nicht als HPV-naiv galt, ergab sich eine Wirksamkeit zur Verhinderung von HPV 16 und 18 assoziierten CIN2+ von 53\% und unabhängig vom HPV-Typ von 30\% (Paavonen et al. 2009, S. 311). Dies bedeutet, die Impfung war auch für Studienteilnehmerinnen, die eine bestehende oder abgelaufene HPV-Infektion hatten, von Vorteil im Vergleich zum Placebo, jedoch insgesamt wesentlich weniger wirksam im Vergleich zur HPV-naiven Studiengruppe. Zusätzlich lässt sich anhand weiterer Daten eine sogenannte Kreuzprotektion durch die Impfung vermuten. Hiermit ist gemeint, dass die Impfung auch gegen andere HPV-Typen als 16 und 18 einen gewissen Schutz bieten kann (STIKO 2009, S. 324ff). Anhand der Daten der FUTURE-Studien und der PATRICIA-Studie sieht die STIKO die Wirksamkeit der HPV-Impfung gegen HPV 16 und 18 assoziierte Zervixkarzinomvorstufen als (STIKO 2009, S. 326). 
Zudem bewertet die STIKO die Sicherheit der beiden Impfungen und stellt fest, dass weder bezüglich Gardasil noch Cervarix schwere unerwünschte Wirkungen im kausalen Zusammenhang mit der Impfung dokumentiert seien (STIKO 2009, S. 326). Ein weiterer Vorteil der Impfung sei die Verhinderung von Operationen zur Diagnose oder Behandlung von Zervixkarzinomvorstufen. Operationen am Gebärmutterhals gehen mit einem erhöhten Risiko für zukünftige Fehlgeburten und Komplikationen in der Schwangerschaft einher (STIKO 2009, S. 320). Diese Risiken könnten also durch die Impfung möglicherweise vermindert werden. Hinsichtlich der hohen Krankheitslast von Zervixkarzinomen in Entwicklungsländern könnte eine HPV-Impfung zudem eine sinnvolle Maßnahme zur Prävention darstellen. Bosch merkt hierzu folgendes an:

„In populations where cytology programmes are either not in place or are not efficient, HPV testing should now be considered and evaluated as an alternative test for primary screening. Prevention of exposure to high risk HPV types by vaccination may prove to be the most efficient and logistically feasible preventive intervention for cervical cancer" (Bosch et al. 2002, S. 244).

Im folgenden sollen Kritikpunkte und offene Fragen genauer beleuchtet werden. Mehrere deutsche WissenschaftlerInnen forderten die HPV-Impfung neu zu bewerten. Ein Grund hierfür ist, dass die Impfstoffe an Frauen im Alter zwischen 15 und 26 getestet wurden, die STIKO jedoch eine Empfehlung für 12 bis 17-jährige ausspricht. Es liegen also keine Daten zur Wirksamkeit für 12 bis 14jährige vor (Dören et al. 2008). Darüber hinaus erläutern sie, dass die HPV-Typen 16 und 18 zwar für $70 \%$ der Zervixkarzinome verantwortlich gemacht werden und die Studien eine hohe Wirksamkeit (bis zu 98\%) gegen Krebsvorstufen assoziiert mit diesen HPV-Typen belegen, dies jedoch nicht bedeute, dass die Krebsfälle um 70\% reduziert würden. Diese zu optimistische Annahme sei bisher noch nicht bewiesen worden (Dören et al. 2008). Die meisten Berechnungen zur letztendlichen Kosteneffektivität der Impfung basieren jedoch auf der Annahme, 70\% der Krebsfälle durch die Impfung reduzieren zu können (Gerhardus und Razum 2010, S. 378). Das Hauptargument gegen die HPV-Impfungen bleibt jedoch die Unsicherheit bezüglich der Wirksamkeit. Diese Unsicherheiten ergeben sich durch die Konzipierung der Studien. Das eigentliche Ziel der Studien, nämlich die Wirksamkeit der Impfstoffe bezüglich der Entstehung von Zervixkarzinomen zu testen, wurde durch einen sogenannten Surrogatparameter ersetzt. In den Studien wurde nämlich die Wirksamkeit der Impfstoffe hinsichtlich der Entstehung von mäßig- bis höhergradigen Krebsvorstufen getestet. (Gerhardus 2009, S. 332). Dies ist zwar sinnvoll, da man aus ethischer Sicht nicht abwarten kann bis sich bei den Studienteilnehmerinnen Zervixkarzinome entwickeln, um dann zu vergleichen, ob dies seltener in der Kohorte der Geimpften vorkommt. Die Entstehung von Zervixkarzinomen ist zudem sehr langsam, sodass man die Studien über mehrere Jahre durchführen müsste. Trotz des in diesem Falle sinnvollen Einsetzens eines Surrogatparameters bleibt also die Ungewissheit, ob durch die Verminderung der Krebsvorstufen auf lange Sicht auch tatsächlich die Krebsfälle reduziert werden. Des Weiteren wird die eigentliche 
Zielgruppe, bestehend aus Mädchen und jungen Frauen vor dem ersten Geschlechtsverkehr, in den Studien durch eine bestimmte Gruppe von Probandinnen ersetæt. Die Gruppe der Probandinnen wurde als HPV-naiv getestet und scheint somit der eigentlichen Zielgruppe am ähnlichsten zu sein. Gerhardus und Razum kritisieren, dass durch den Einsatz von Surrogatparametern die Studienergebnisse keine zuverlässigen Schlüsse bezüglich der Wirksamkeit der Impfungen zulassen würden (Gerhardus und Razum 2010). Darüber hinaus ist bisher ungeklärt, ob durch den Wegfall von HPV-Typen 16 und 18 mithilfe der Impfung andere seltenere onkogene HPV-Typen vermehrt auftreten könnten (sogenanntes Replacement-Phänomen) (STIKO 2009, S. 326; Arzneitelegramm 2007). Zudem gibt es die Sorge, eine HPV-Erstinfektion nach dem Verlust der Impfwirkung könnte im höheren Alter mit einem schwereren Verlauf einhergehen, wie dies beispielsweise bei Windpocken der Fall ist (Arzneitelegramm 2007). Des Weiteren nennt die STIKO im Zuge ihrer Begründung für die Impfempfehlung einige bisher offen gebliebene Fragen bezüglich der HPV-Impfung. Es wird beispielsweise daraufhin gewiesen, dass die Dauer des Impfschutzes beziehungsweise die Notwendigkeit einer Wiederholung der Impfung noch nicht geklärt wurde (STIKO 2009, S. 326). Außerdem räumt die STIKO ein, dass noch offen ist, ob die Impfung tatsächlich die Krankheitslast von Zervixkarzinomen senkt. Zudem bleibt unklar, inwiefern eine Impfung auch für Jungen sinnvoll sein könnte. Zusätzlich stellt die STIKO die Wirksamkeit der beiden Impfstoffe hinsichtlich der Reduzierung von Zervixkarzinomvorstufen unabhängig vom HPVTyp infrage. Offen bleibt laut der STIKO auch die Sicherheit der Impfstoffe bezüglich seltener unerwünschter Wirkungen. Außerdem stellt die STIKO die Frage, ob das Angebot der Impfung die Wahrnehmung der Früherkennungsmaßnahmen und deren Qualität beeinflussen könnte. Dahinter verbirgt sich vermutlich die Sorge, Untersuchungen zur Früherkennung würden weniger in Anspruch genommen, da geimpfte Frauen womöglich keine Notwendigkeit mehr darin sähen. Die STIKO weist somit in ihrer Impfempfehlung aus dem Jahre 2012 explizit darauf hin, dass die Impfungen nicht gegen alle onkogenen HPV-Typen schützen und alle geimpften Personen weiterhin die Früherkennungsuntersuchungen wahrnehmen müssen (STIKO 2012, S. 4). Zusätzlich zu den bisher genannten Anmerkungen, die beide Impfstoffe betreffen, sollen im Folgenden noch weitere spezifische Kritikpunkte hinsichtlich der PATRICIA-Studie (Cervarix) dargstellt werden. Diese sind wichtig, um die Fallstudie besser zu verstehen. Zum einen wurde bei dieser Studie als Placebo für die Kontrollgruppe ein Hepatitis-A-Impfstoff verwendet, zu dem jedoch keine weiteren Informationen vorliegen (Arzneitelegramm 2009). Da aktuell allerdings nur ein reiner Hepatitis-A-Impfstoff von GSK auf dem Markt ist, nämlich Havrix 1440, kann davon ausgegangen werden, dass dieser als Placebo verwendet wurde (Paul-Ehrlich-Institut 2013). Aus der Fachinformation zu Havrix 1440 geht hervor, dass auch dieser Impfstoff genau wie Cervarix als Adjuvans Aluminiumhydroxid enthält (GlaxoSmithKline 2004). Um valide Aussagen über die Verträglichkeit von Cervarix treffen zu können, hätte man jedoch die HPV-Impfung im Vergleich zu keiner Impfung, also beispielsweise Kochsalzlösung als Placebo, testen müssen. Im Arzneitelegramm wird hierzu folgendes angemerkt: 
„Das Ergebnis der Studie, dass sich die Raten unerwünschter Effekte einschließlich schwerwiegender Ereignisse, chronischer oder Autoimmunerkrankungen in beiden Gruppen nicht unterschieden haben, bleibt daher ohne Aussagekraft" (Arzneitelegramm 2009, S. 72).

Es besteht zudem die Befürchtung, dass durch die Anwendung von Cervarix Autoimmunerkrankungen zunehmen könnten (Arzneitelegramm 2009). Im Vergleich zu Gardasil schützt Cervarix nicht vor HPV-Typ 6 und 11 (verursachen Genitalwarzen). Dies könnte jedoch auch als Vorteil bewertet werden, da es Hinweise gibt, dass Infektionen mit HPV 6 oder 11 vor Zervixkarzinomen durch HochrisikoHPV-Typen schützen sollen (Arzneitelegramm 2007). Darüber hinaus scheint Cervarix eine höhere Kreuzprotektion gegenüber anderen HPV-Typen als 16 und 18 im Vergleich zu Gardasil aufzuweisen. Um unter anderem dies ausreichend zu belegen, wird im Arzneimitteltelegramm eine Studie zum Vergleich von Cervarix und Gardasil gefordert (Arzneitelegramm 2009).

\section{Exkurs: Skandal: HPV-Impfstudie in Indien}

Vor Beginn der eigentlichen Analyse der Fallstudie muss zum besseren Verständnis an dieser Stelle eine in Indien durchgeführte HPV-Impfstudie beleuchtet werden, die aus ethischer Sicht als Skandal bezeichnet werden kann. Hierbei handelt es sich um eine Studie, die 2009 vom Gesundheitsministerium des Bundesstaates Andrha Pradesh, dem Indian Council of Medical Research und der Nichtregierungsorganisation PATH (Program for Appropriate Technology in Health) initiiert wurde. Insgesamt sollten 16.000 Mädchen im Alter zwischen 10 und 14 Jahren gegen HPV geimpft werden (BUKO 2010a; Srinivasan 2011). Nachdem es zu sieben Todesfällen unter den Studienteilnehmerinnen kam, wurde die Studie vorzeitig beendet (Srinivasan 2011). Untersuchungen zufolge standen die Todesfälle in keinem kausalen Zusammenhang zur Impfung (BUKO 2010a; Srinivasan 2011). Allerdings brachte eine genauere Prüfung einige Missstände bezüglich der Aufklärung und des informed consent zutage (BUKO 2010a). Es gibt beispielsweise Hinweise, dass viele der Mädchen nur ungenügend aufgeklärt wurden. Grundlegende Fakten, die zum Verständnis einer HPV-Impfung wichtig sind, wie beispielsweise was die Zervix ist, waren ihnen unbekannt (BUKO 2010a). Des Weiteren wurde kritisiert, dass die Studie an einer vulnerablen Gruppe durchgeführt wurde, da die meisten Mädchen in armen Verhältnissen lebten und viele weder lesen noch schreiben konnten (Srinivasan 2011). Ein Teil der Mädchen lebte in Internaten und meist wussten die Eltern nicht, dass ihre Töchter an einer medizinischen Studie teilnahmen, denn die Zustimmung wurde teilweise von den LeiterInnen der Internate eingeholt (Srinivasan 2011). Zudem wurde die Tatsache, dass es sich um eine medizinische Studie handelte, dadurch verschleiert, dass das Gesundheitsministerium involviert war. Einige Eltern hielten die Studie daher für eine Impfkampagne. Ein weiterer Kritikpunkt ist, dass während der Studie potenzielle schwerwiegende unerwünschte Wirkungen der Impfung nicht adäquat dokumentiert wurden. Darüber hinaus waren die Studienteilnehmerinnen über die Laufzeit der Studie nicht versichert (Srinivasan 2011). 


\subsubsection{Zielsetzung und Methodik der Fallstudie B}

Im folgenden Abschnitt soll auf der Basis der Hintergrundinformationen und des aktuellen Forschungsstands die Zielstellung der Fallstudie beleuchtet werden. In der Publikation zur Studie von Bhatla et al. werden drei Hauptgründe für die Initiierung der Studie genannt (Bhatla et al. 2010). Zuerst stellen Bhatla et al. die enorm hohe Krankheitslast von Zervixkarzinomen für Frauen in Indien dar. Diese zeige sich beispielsweise durch eine hohe altersstandardisierte Inzidenzrate von 30,7 und Mortalitätsrate von 17,4 pro 100.000 Frauen. Als zweiter Grund werden von Bhatla et al. die Schwierigkeiten bei der Einführung flächendeckender Zervixkarzinom-Screening-Programme angegeben. Als dritte Begründung für die Durchführung der Studie führen die AutorInnen Studiendaten an, die zeigen, dass schätzungsweise 75\% der Zervixkarzinomfälle in Indien mit HPV 16 und 18 assoziiert sind. Durch diese Gegebenheiten wachse ein lokales Interesse mithilfe von HPVImpfungen die Krankheitslast von Zervixkarzinomen kontrollieren zu können. Ziel der Studie war es, die Immunogenität und die Sicherheit der HPV-16/18 AS04-Adjuvans-Impfung (Cervarix) für gesunde indische Frauen im Alter von 18 bis 35 Jahren zu testen (Bhatla et al. 2010, S. 123).

Die Studie wurde doppelt blind, randomisiert und Placebo-kontrolliert durchgeführt und war eine Phase IIIb-Studie. Das Ziel von Phase III-Studien ist es, die Wirksamkeit einer Intervention in großen Gruppen von 100en bis 1000en ProbandInnen zu überprüfen. Dies geschieht mithilfe eines Vergleichs der zu prüfenden Intervention mit anderen üblichen oder experimentellen Interventionen. Des Weiteren sollen in einer Phase III-Studie unerwünschte Ereignisse aufgezeichnet werden und Informationen gesammelt werden, die es möglich machen, die neue Intervention in Zukunft sicher anwenden zu können (WHO 2014).

Die Probandinnen wurden mit einem Verhältnis von 1:1 in zwei verschiedene Gruppen aufgeteilt. Hieraus ergaben sich eine Impf- und eine Placebogruppe. Die Impfgruppe erhielt den HPV-16/18 AS04-Adjuvans Impfstoff (Cervarix) und die Placebogruppe Aluminumhydroxid als Placebo. Beide Kohorten wurden an Tag null, nach einem und nach sechs Monaten geimpft. Bis einen Monat nach Abschluss der Impfserie wurden die Studienteilnehmerinnen noch nachbetreut, ein Follow-up bis zu Monat sieben war also gewährleistet (Bhatla et al. 2010, S. 123).

Ein Ziel der Studie war es, die Immunogenität des HPV-Impfstoffs zu testen, also herauszufinden, ob das Immunsystem der Probandinnen auf die Impfung mit der Bildung von Antikörpern gegen HPV 16 und 18 reagieren würde. Hierfür wurde den Studienteilnehmerinnen vor Beginn der Impfserie und sieben Monate später bei der letzten Untersuchung Blut entnommen. Die Blutproben wurden zentrifugiert und das Serum wurde aufbewahrt und nach Belgien in Laboratorien von GSK Biologicals verschickt, wo die Proben ausgewertet wurden. Hierbei wurde als Methode für die Messung der Höhe des HPV-16/18-Antikörpertiters, also die Menge der Antikörper pro Milliliter Serum der jeweiligen Probandin, Enzyme-linked Immunosorbent Assays (ELISA) verwendet (Bhatla et al. 2010, S. 125). 
Die Prüfung der Sicherheit der Impfung als zweite Zielstellung der Studie wurde anhand von Erfragen und Dokumentieren bestimmter Symptome eruiert. Diese wurden eingeteilt in lokale und allgemeine Symptome. Schmerz, Rötung und Schwellung an der Einstichstelle zählten hierbei zu den lokalen Symptomen. Unter allgemeine Symptome fielen folgende Beschwerden: Fieber, Kopfschmerzen, Müdigkeit, gastrointestinale Beschwerden, wie Übelkeit, Erbrechen, Diarrhö und abdominale Schmerzen, Gelenk- und Muskelschmerzen sowie allergische Reaktionen wie Hautausschlag und Urtikaria (Bhatla et al. 2010, S. 125). Die Symptome wurden jeweils sieben Tage nach einer Impfung, also von Tag null bis Tag sechs, mithilfe von sogenannten diary cards abgefragt und dokumentiert. Die Intensität der Symptome wurde hierbei in verschiedene Schweregrade von eins bis drei eingeteilt, wobei das Ausmaß an Unannehmlichkeit für die jeweilige Probandin als ausschlaggebend galt. Symptome, die als Schweregrad drei eingestuft waren, wurden folgendermaßen definiert:

- Schmerz, der normale alltägliche Aktivitäten einschränkte

- Schwellung oder Rötung der Einstichstelle mit einem Durchmesser über 50 mm

- axillär gemessenes Fieber über $39,0^{\circ} \mathrm{C}$ oder

- andere Symptome, die durch ihre Intensität das Ausüben normaler Aktivitäten verhinderten (Bhatla et al. 2010, S. 125)

Unerwünschte Wirkungen, die nicht mit den diary cards abgefragt wurden, wurden bis zu 30 Tage nach der Impfung weiter verfolgt. Schwangerschaften und schwerwiegende Nebenwirkungen wurden über die komplette Studienlaufzeit verfolgt und letztere folgendermaßen eingeteilt:

- Serious adverse events (SAE): schwerwiegende unerwünschte Ereignisse. Hierzu zählen: Tod, lebensbedrohliche Situation, Klinikeinweisung oder verlängerter Klinikaufenthalt, Behinderung oder dauerhafter Schaden, angeborene Anomalien oder Geburtsfehler, Notwendigkeit einer Intervention zur Verhinderung dauerhafter Beeinträchtigung oder Schaden oder andere schwerwiegende medizinische Ereignisse.

- New-onset chronic disorders (NOCD): neu aufgetretene chronische Erkrankungen, wie beispielsweise Diabetes mellitus, Asthma bronchiale, Allergien oder Autoimmunerkrankungen.

- Medically significant conditions (MSC): medizinisch bedeutsame Ereignisse, definiert als unerwünschte Ereignisse, sogenannte adverse events (AE), die das Aufsuchen einer Notaufnahme oder einen Arztbesuch unabhängig von einer gewöhnlichen oder bereits bekannten Erkrankung, einer Routineuntersuchung oder Impfung notwendig machte.

Anhand der ausgefüllten diary cards wurde verglichen, ob bestimmte Symptome häufiger oder schwerwiegender in der Impfgruppe oder in der Placebogruppe auftraten und hiermit die Sicherheit der Impfung getestet (Bhatla et al. 2010, S. 125). 


\subsubsection{Bedingungen und Studienteilnehmerinnen der Fallstudie B}

Nach der Betrachtung der Zielstellung und Methodik der Fallstudie folgt nun die Darstellung allgemeiner Bedingungen hinsichtlich der Studie. Diese sind die Einschlusskriterien für eine Studienteilnahme sowie Informationen über die Studienteilnehmerinnen. Bei der Fallstudie handelt es sich um eine multizentrische Studie, durchgeführt an vier verschiedenen Studienzentren in Indien (Chandigarh, Kolkata, Mumbai, New Delhi) (ClinicalTrials.gov 2011). Die Studie wurde von GSK Biologicals finanziert, die den zu testenden HPV-Impfstoff Cervarix entwickelten. Vor Beginn der Studie wurde das Studienprotokoll vom Drugs Controller General of India genehmigt. Außerdem erteilten das jeweils zum Studienzentrum zugehörige wissenschaftliche Komitee sowie das entsprechende Ethikkomitee ihre Zustimmung zur Durchführung der Studie (Bhatla et al. 2010). Die Einschlusskriterien für eine Studienteilnahme waren folgende:

- Einschätzung des Forscherteams, dass die Probandinnen die Bedingungen des Studienprotokolls wahrscheinlich einhalten können und werden

- Frauen im Alter zwischen 18 und 35 Jahren

- schriftliche informierte Zustimmung der Probandinnen

- guter Gesundheitsstatus

- negativer Schwangerschaftstest (Urintest)

- gebärfähige Probandinnen müssen zustimmen 30 Tage vor und zwei Monate nach der Impfserie keinen Geschlechtsverkehr zu haben oder adäquate Verhütungsmittel zu verwenden (ClinicalTrials.gov 2011)

Für den Ausschluss einer Studienteilnahme wurden unter anderen folgende Kriterien angewendet:

- Einnahme eines experimentellen oder noch nicht registrierten Medikaments oder Impfung

- schwangere oder stillende Frauen

- dauerhafte Einnahme von Immunsuppressiva oder Vorliegen einer Immunschwäche

- bereits erfolgte Impfung gegen HPV

- bisherige allergische Reaktionen, hervorgerufen durch einen der Inhaltsstoffe der Impfung (ClinicalTrials.gov 2011)

Folgende Infornationen konnten über die Probandinnen ermittelt werden: Die Studienteilnehmerinnen waren Frauen mit indischer Staatsangehörigkeit im Alter zwischen 18 und 35 Jahren. Das Durchschnittsalter betrug 28,4 Jahre (Bhatla et al. 2010, S. 126). In der Publikation sind keine weiteren Informationen über die Probandinnen angegeben. Daher wurden die AutorInnen und das betreffende Journal kontaktiert. In einer Antwort wurde der sozioökonomische Status der Probandinnen als der unteren Mittelklasse bis Mittelklasse angehörig beschrieben. ${ }^{12}$ Hierbei könnte es von Bedeutung sein, ob

12 Persönliche Kommunikation mit Erstautorin (Email vom 15.09.2012) 
festgehalten wurde, welcher Kaste die jeweiligen Probandinnen möglicherweise angehören oder ob es unüblich ist, dies im Rahmen der Erhebung persönlicher Daten der StudienteilnehmerInnen zu erfragen. Der Bildungsstatus der Probandinnen wird variierend von Analphabetinnen bis hin zu Frauen, die als Fachpersonal bezeichnet werden, angegeben. ${ }^{13}$ Es gibt allerdings, auch auf gezielte Nachfrage hin, keine genaueren Informationen dazu, wie viele der Probandinnen Analphabetinnen waren oder was genau mit Fachpersonal gemeint ist oder welchen Schulabschluss die Probandinnen hatten oder wie viele Jahre sie die Schule besuchten. Des Weiteren wurde nicht erläutert, wie sichergestellt wurde, dass der informed consent-Prozess für die Analphabetinnen angemessen ist. Zusätzlich könnte die Einsicht in die Aufklärungsdokumente aufschlussreiche Informationen zur Qualität des informed consent bieten. Die Bitte für diese Arbeit die informed consent-Dokumente zur Verfügung zu stellen, wurde jedoch nicht erfüllt. Darüber hinaus könnte die Form der Rekrutierung Hinweise auf eine mögliche Vulnerabilität der Probandinnen geben. Hierbei wäre wichtig zu wissen, ob die Probandinnen beispielsweise von ihren behandelnden ÄrztInnen auf die Studie hingewiesen wurden oder aber durch ein Plakat oder Flyer auf die Möglichkeit einer Studienteilnahme aufmerksam wurden. Hierzu wurden jedoch trotz gezielter Nachfrage keine Angaben von der Autorin gemacht. Zudem wäre es bedeutsam zu wissen, ob den Probandinnen eine Aufwandsentschädigung gezahlt wurde und wenn ja, welche Summe. Die Möglichkeit, die Studienteilnehmerinnen könnten aufgrund eines unangemessenen Anreizes zugestimmt haben, könnte hiermit genauer eingeschätzt werden. Insgesamt fehlen folglich viele Informationen, die wichtig wären, um eine mögliche Vulnerabilität der Probandinnen gänzlich einschätzen zu können.

\subsubsection{Verlauf und Ergebnisse der Fallstudie B}

In der Publikation zur Studie wird beschrieben, dass diese in Übereinstimmung mit den ethischen Grundsätzen der Deklaration von Helsinki und gemäß den Standards der Indian Good Clinical Practices, veröffentlicht von der Central Drugs Standard Control Organization, durchgeführt wurde. Nach Abschluss der Studie wurde von den SponsorInnen überprüft, ob diese mit den Standards des Good Clinical Practice, wie sie vom International Council of Harmonization verlangt werden, durchgeführt wurde. Hierbei ergaben sich keine kritischen Erkenntnisse (Bhatla et al. 2010, S. 124).

Die Rekrutierung für die Studie begann im Juli 2006 und wurde im März 2007 beendet. Im Dezember 2007 fanden die letzten Follow-up-Untersuchungen statt (Bhatla et al. 2010, S. 124). Die Studie wurde im Juni 2006 bei clinicaltrials.gov registriert (ClinicalTrials.gov 2011).

13 Persönliche Kommunikation mit Erstautorin (Email vom 15.09.2012) 
Die Studienteilnehmerinnen wurden in verschiedene Gruppen eingeteilt. Die Total Vaccinated Cohort (TVC) enthält alle Probandinnen, die rekrutiert, randomisiert und mindestens einmalig geimpft wurden (Impfstoff oder Placebo) und belief sich insgesamt auf 354 Probandinnen. Hiervon wurden 176 in die Impfgruppe und 178 Probandinnen in die Placebogruppe eingeteilt. Insgesamt beendeten 330 Probandinnen die Studie und erhielten somit alle drei Dosen der Impfung oder des Placebos (Bhatla et al. 2010, S. 127). Die Compliance bezüglich der Verabreichung aller drei Impfdosen beziehungsweise Placebodosen betrug über 97\%. In der Impfgruppe beendeten 93,2\% (164/176) und in der Placebogruppe 94,4\% (168/178) der Probandinnen die Studie. Gründe für den vorzeitigen Abbruch der Studie waren Widerspruch der Zustimmung (unabhängig von unerwünschten Nebenwirkungen), Umzug in eine andere Region, persönliche Gründe, Schwangerschaften und eine Fehlgeburt sowie das Nicht-Einhalten der kompletten Impfserie (Bhatla et al. 2010, S. 127).

Für die Prüfung der Sicherheit der Impfung wurde eine sogenannte According-to-protocol (ATP) safety cohort eingeteilt, die zunächst alle Studienteilnehmerinnen miteinbezog, jedoch Probandinnen ausschloss, die schwanger wurden oder gegen die Vorgaben im Protokoll verstießen, wie beispielsweise eine zusätzliche Impfung während der Studienzeit erhielten. Insgesamt betrug die ATP safety cohort 167 in der Impfgruppe und 170 Probandinnen in der Placebogruppe (Bhatla et al. 2010, S. 127).

Außerdem wurde noch eine ATP immunogenicity cohort benannt, die Probandinnen miteinbezog, die die Auswahlkriterien erfüllten und die Regelungen des Protokolls befolgten. Ausgeschlossen aus dieser Kohorte wurden Probandinnen, wenn essentielle Daten ihrer Serologie fehlten (Impfgruppe: 10 und Placebogruppe: 8), aufgrund von Erkrankungen, die laut Protokoll nicht zulässig waren oder wenn der Ablaufplan der Blutentnahmen nicht eingehalten wurde. Daraufhin ergab sich eine ATP-immunogenicity cohort, also die Gruppe von Probandinnen deren Antikörpertiter zur Feststellung der Immunogenität der Impfung gemessen und analysiert wurde, von 153 in der Impfgruppe und 159 in der Placebogruppe (Bhatla et al. 2010, S. 127).

Bezüglich des Studienverlaufs sollen an dieser Stelle zudem Informationen über aufgetretene Nebenwirkungen und Schwangerschaften während der Studienlaufzeit dargestellt werden. Während der Studie gab es sechs Serious adverse events (SAE). Zwei der SAEs traten in der Impfgruppe auf. Hierbei lagen zum einen eine Appendizitis und zum anderen eine Lymphknotentuberkulose vor. $\mathrm{Zu}$ den vier SAEs der Placebogruppe zählten eine bronchogene Zyste, ein Fall von Katarakt, eine Fehlgeburt und ein Pneumothorax der linken Lunge. Außerdem wurden zwei neu aufgetretene chronische Erkrankungen (NOCD) festgestellt. Beide Fälle, zum einen Hypersensitivität und zum anderen Urtikaria, traten in der Placebogruppe auf. Des Weiteren traten bei 13 Probandinnen der Impfgruppe (7,4\%) und 24 der Placebogruppe (13,5\%) medizinisch bedeutsame Ereignisse (MSC) auf. Eine genauere Beschreibung über die Entität der MSC ist nicht dargelegt (Bhatla et al. 2010, S. 126ff).

Vor jeder Impfung oder Verabreichung des Placebos wurden die Probandinnen nach einer vorliegenden Schwangerschaft gefragt und es wurde jeweils ein Schwangerschaftstest (Urintest) durchgeführt. 
Während der gesamten Studienlaufzeit wurden zehn Schwangerschaften dokumentiert. Neun dieser Probandinnen entschieden sich aufgrund abgeschlossener Familienplanung für einen Schwangerschaftsabbruch (Impfgruppe: 8 und Placebogruppe: 1). Eine der Schwangerschaften führte zu einer Fehlgeburt (Placebogruppe) und wurde bereits im Kontext der SAEs erwähnt (Bhatla et al. 2010, S. $125 \mathrm{ff})$

\section{$\underline{\text { Studienergebnisse }}$}

In diesem Abschnitt sollen zunächst die Ergebnisse bezüglich der Immunogenität der HPV-Impfung und im Anschluss die Auswertung hinsichtlich der Sicherheit der Impfung erläutert werden. Es wurden 166 Serumproben von Probandinnen aus der Impfgruppe und 170 aus der Placebogruppe analysiert. Die Messung der Antikörper vor Beginn der Impfserie an Tag null ergab, dass 70,8\% der Studienteilnehmerinnen der Impfgruppe und 74,2\% der Studienteilnehmerinnen in der Placebogruppe einen negativen Serostatus bezüglich HPV 16 und 18 aufwiesen. Nur einen der beiden HPV-Typen betreffend lag bei 91\% der Probandinnen der Impfgruppe und bei 95\% der Placebogruppe ein negativer Serostatus vor. Bei der letzten Follow-up-Untersuchung einen Monat nach der letzten Impfung waren 100\% der Teilnehmerinnen der Impfgruppe serokonvertiert. Dies bedeutet, dass diese Probandinnen, die vor Beginn der Impfserie seronegativ bezüglich HPV 16 und 18 waren und danach seropositiv wurden, folglich Antikörper gegen HPV 16 und 18 gebildet hatten. In der Placebogruppe zeigte sich nur bei 11,5\% eine Serokonversion hinsichtlich HPV-16-Antikörpern und bei 3,2\% hinsichtlich HPV-18-Antikörpern. Des Weiteren wurde bestätigt, dass alle Probandinnen der Impfgruppe, die seropositiv bezüglich HPV 16 und 18 vor Beginn der Impfserie waren, auch bei der letzten Follow-up-Untersuchung seropositiv waren. Allerdings waren die Antikörpertiter bei den ursprünglich seropositiven Probandinnen der Impfgruppe nach der Impfserie wesentlich höher als zuvor. Dieser Effekt konnte bei den Probandinnen der Placebogruppe, die zu Beginn der Studie seropositiv waren, nicht nachgewiesen werden. Hier ergaben die Antikörpertiter im Vergleich vor und nach der Impfserie circa die gleichen Werte. Es lässt sich zusammenfassend festhalten, dass die Immunogenität der Impfung Cervarix in der Fallstudie als positiv zu bewerten ist (Bhatla et al. 2010, S. 126ff).

Bei der Prüfung der Sicherheit der HPV-Impfung zeigte sich eine Compliance bezüglich der Rückgabe der diary cards von 97,5\% in der Impfgruppe und 98,1\% in der Placebogruppe. Lokale Symptome, wie Schmerzen an der Einstichstelle, gaben 80,1\% der Probandinnen der Impfgruppe und 60,3\% der Placebogruppe an. Hierbei ordneten 20,5\% der Impfgruppe und 4\% Probandinnen der Placebogruppe die Schmerzen dem Schweregrad drei zu. Weitere lokale Symptome wie Rötung und Schwellung der Einstichstelle wurden insgesamt seltener genannt als Schmerz. Die Symptome wurden jedoch jeweils häufiger in der Impfgruppe im Vergleich zur Placebogruppe dokumentiert. Rötung wurde beispielsweise von 32,7\% der Probandinnen der Impfgruppe, von 13,8\% der Placebogruppe und 
Schwellung von 40,4\% der Probandinnen der Impfgruppe und 20,1\% der Placebogruppe angegeben. Die Abfrage allgemeiner Symptome ergab eine ähnliche Verteilung in der Impfgruppe im Vergleich zur Placebogruppe. Hierbei waren Müdigkeit, Kopfschmerzen und Fieber die am häufigsten aufgetretenen Symptome. Allgemeine Symptome mit Schweregrad drei wurden in beiden Gruppen selten angegeben und es zeigte sich kein bedeutsamer Unterschied zwischen den beiden Gruppen (Impfgruppe: unter 6,4\% und Placebogruppe: unter 5,7\%). Allgemeine Symptome, die vom Forscherteam als in Zusammenhang zur Impfung beziehungsweise zur Verabreichung des Placebos stehend bewertet wurden, traten in beiden Gruppen mit ähnlicher Häufigkeit auf (Impfgruppe: unter 38\% und Placebogruppe: unter 35,1\%). Unerwünschte Nebenwirkungen, die nicht explizit mit den diary cards abgefragt wurden, traten bei 11,4\% der Probandinnen der Impfgruppe und 14,6\% der Placebogruppe auf. Die Symptome wurden bei 2,8\% der Studienteilnehmerinnen der Impfgruppe und 2,2\% der Placebogruppe als in Zusammenhang mit der Impfung beziehungsweise mit dem Placebo stehend eingestuft. Bezüglich der sechs aufgetretenen schwerwiegenden unerwünschten Ereignissen, die bereits weiter oben erläutert wurden, gaben die AutorInnen an, dass hierbei jeweils kein Zusammenhang zur Impfung oder zum Placebo hergestellt werden konnte (Bhatla et al. 2010, S. 126f). 


\subsection{Interpretation der Fallstudie B im Hinblick auf Vulnerabilität der}

\section{Studienteilnehmerinnen}

Im Anschluss an die deskriptive Darstellung der HPV-Impfstudie soll in diesem Kapitel die normative Bewertung der Analyse folgen. Hierzu müssen zunächst einige zusätzliche Informationen und Fragen erläutert und diskutiert werden, die mit Blick auf ethische Konflikte im Kontext der Studie noch offen geblieben sind. In einem zweiten Schritt gilt es, sowohl mithilfe der Zusatzinformationen als auch der Analyse die Fallstudie auf eine mögliche Vulnerabilität der Studienteilnehmerinnen zu überprüfen.

\subsubsection{Ethische Aspekte bei der Durchführung von Fallstudie B}

Ethische Aspekte betreffend bleiben bei der Durchführung der Fallstudie letztendlich einige Fragen offen. Zum einen sind dies Fragen, die sich direkt auf die Studie beziehen, beispielsweise methodische Vorgehensweisen sowie Risiken und Nutzen für die Studienteilnehmerinnen. Zum anderen wirft die Studie indirekt Fragen zum Nutzen von HPV-Impfungen für Frauen in Indien auf.

Wie schon bezüglich der PATRICIA-Studie angemerkt, ist auch bei der Fallstudie der Grund für die Verwendung von Aluminiumhydroxid als Placebo unklar. Das Adjuvans des getesteten HPV-Impfstoffs enthält auch Aluminiumhydroxid und somit scheint dies als Placebo ungeeignet (Exley 2011). Mögliche unerwünschte Nebenwirkungen, die durch Aluminiumhydroxid hervorgerufen werden könnten, würden nämlich in der Impfgruppe und in der Placebogruppe mit einer ähnlichen Häufigkeit auftreten. Somit sind Aussagen bezüglich der gleichen Verteilung unerwünschter Nebenwirkungen in der Impfgruppe im Vergleich zur Placebogruppe unbrauchbar und unerwünschte Nebenwirkungen, die durch das Adjuvans der Impfung hervorgerufen werden könnten, können nicht ausgeschlossen werden. Hierdurch sind folglich die Ergebnisse der Studie, die die Prüfung der Sicherheit des Impfstoffs positiv bewerten, fragwürdig. Die Nachfrage nach den Gründen für die Verwendung von Aluminiumhydroxid als Placebo blieb von der Autorin der Studie unbeantwortet.

Darüber hinaus könnte das relativ kurze Follow-up von nur einem Monat nach Verabreichung der letzten Impfdosis kritisiert werden. Hierdurch werden mögliche chronische Erkrankungen oder andere unerwünschte Nebenwirkungen, die erst im längerfristigen Verlauf auftreten könnten, nicht mehr erfasst.

Des Weiteren wäre es hilfreich zu wissen, ob den Probandinnen für die Laufzeit der Studie Konzeptionsschutz zur Verfügung gestellt wurde oder inwiefern die Studienteilnehmerinnen hierfür selbstverantwortlich waren. Hierzu wird von der Autorin angegeben, dass die meisten Verhütungsmittel kostenfrei vom Ministry of Health and Family Welfare zur Verfügung gestellt werden. Inwiefern die Probandinnen dies wissen oder von den ForscherInnen eigens darüber informiert wurden, ist unklar. 
In den International Ethical Guidelines for Biomedical Research Involving Human Subjects der CIOMS wird folgendes festgelegt:

„In this discussion, if participation in the research might be hazardous to a fetus or a woman if she becomes pregnant, the sponsors/investigators should guarantee the prospective subject a pregnancy test and access to effective contraceptive methods before the research commences" (CIOMS 2002, S. 72 ).

Hierzu stellt sich zusätzlich die Frage, wie sichergestellt wurde, dass die Probandinnen über die korrekte Anwendung von möglichen Verhütungsmitteln ausreichend informiert waren. Diese Fragen ergeben sich unter anderem, da während der Studie zehn Schwangerschaften dokumentiert wurden, obwohl gebärfähige Probandinnen laut Protokoll dazu verpflichtet waren, sicheren Konzeptionsschutz anzuwenden oder abstinent hinsichtlich sexueller Kontakte zu leben.

Zusätzlich ist der Nutzen einer Impfung für Frauen im Alter der Probandinnen, nämlich zwischen 18 und 35 Jahren, fragwürdig. Die Autorin erläutert hierzu, dass diese Altersgruppe gewählt wurde, um mit vorherigen Studien vergleichbar zu sein und dass dies zudem den Forderungen der zuständigen Behörden entsprach. ${ }^{14}$ Die Ergebnisse der PATRICIA-Studie zeigen, dass Cervarix vor allem eine hohe Wirksamkeit zur Verhinderung von Zervixkarzinomvorstufen für Mädchen und junge Frauen vor dem ersten Geschlechtsverkehr aufweist. Bei den Probandinnen der Fallstudie mit einem Durchschnittsalter von 28 Jahren muss jedoch davon ausgegangen werden, dass die meisten Studienteilnehmerinnen sexuell aktiv sind. Hierdurch ist der Nutzen der Impfung für die Studienteilnehmerinnen hinsichtlich der Verhinderung von Krebsvorstufen als relativ gering einzuschätzen. Es ist allerdings unklar, ob GSK Biologicals, die sowohl die PATRCIA- als auch die hier analysierte Fallstudie finanzierte, zum Zeitpunkt der Fallstudie bereits Ergebnisse aus der PATRICIA-Studie vorlagen. Diese Ergebnisse zeigen, dass Cervarix für bereits sexuell aktive Frauen von geringem Nutzen ist. Die PATRICIA-Studie wurde 2004 begonnen und Cervarix in Deutschland im Juli 2007 zugelassen. Die Fallstudie wurde im Juli 2006 begonnen und im Dezember 2007 abgeschlossen. Folglich ist es durchaus denkbar, dass es eine zeitliche Überschneidung gab und bereits Ergebnisse aus der PATRICIA-Studie vorlagen, zu einem Zeitpunkt, an dem diese Fallstudie noch nicht abgeschlossen war. Dies würde bedeuten, dass die Fallstudie eventuell weitergeführt wurde, obwohl bereits bekannt war, dass die Impfung für die Probandinnen von geringem Nutzen sein könnte.

Hinsichtlich des Nutzens einhergehend mit einer Studienteilnahme stellt sich zusätzlich die Frage, warum den Probandinnen im Rahmen der Studie keine Screening-Untersuchung zur Prävention von Zervixkarzinomvorstufen angeboten wurde. Eine diesbezügliche Stellungnahme von der Autorin blieb bisher aus. Die Probandinnen förderten mit ihrer Studienteilnahme Forschung mit dem Fernziel die Krankheitslast von Zervixkarzinomen für Frauen in Indien zu senken, wobei ihnen selbst die in westlichen Ländern bereits etablierten Methoden zur Früherkennung vorenthalten wurden. Zudem hätten solche Screening-Untersuchungen zu Beginn der Studie und bei der letzten Follow-up-

14 Persönliche Kommunikation mit Erstautorin (Email vom 15.09.2012) 
Untersuchung nach Abschluss der Impfserie eine höhere Aussagekraft bezüglich der eigentlichen erwünschten Wirkung der Impfung, nämlich der Verhinderung von Krebsvorstufen, gehabt.

Nun sollen einige Aspekte, die HPV-Impfungen im Allgemeinen in Indien betreffen und somit indirekt die Zielstellung der Fallstudie in Frage stellen könnten, erläutert werden. Zunächst muss abermals darauf hingewiesen werden, dass die tatsächliche Wirksamkeit der HPV-Impfungen zur Verhinderung von Zervixkarzinomen noch nicht abschließend geklärt ist (siehe hierzu Kapitel 5.3.1). Somit bleibt der Nutzen einer HPV-Impfung für Frauen in Indien fragwürdig. Zusätzlich gibt es die Sorge, dass die Kosten einer HPV-Impfung einen limitierenden Faktor für den flächendeckenden Einsatz der Impfung in Indien darstellt. Die momentanen (2010) Kosten einer dreifachen HPV-Impfung in Indien liegen bei etwa 9000 Rupien. Da weniger als 50\% der Kinder gegen Diphterie, Pertussis und Tetanus in Indien geimpft werden, was nur circa drei Rupien kostet, stellt sich folglich die Frage, wie in Indien flächendeckende HPV-Impfungen finanziert werden könnten (Sama Resource Group for Women and Health 2010).

Des Weiteren ergeben sich einige Probleme bezüglich einer HPV-Impfung in Indien, die vor allem gesellschaftliche Aspekte betreffen. Hierbei entsteht beispielsweise die Sorge, dass die Impfung als Alternative zum Screening zur Prävention von Zervixkarzinomen verstanden werden könnte. Es könnte beispielsweise ein öffentliches Bewusstsein fehlen, dass die Impfung nicht gegen alle onkogenen HPVTypen wirksam ist und dass zudem die Dauer des Impfschutzes noch nicht geklärt ist, wodurch ein Screening weiterhin notwendig bleibt (Kang 2010). Zudem wird kritisiert, dass in Regionen Indiens mit einer hohen Krankheitslast von Zervixkarzinomen, Frauen häufig nur eingeschränkt Zugang zu Screening Programmen haben. Somit wäre vermutlich gerade in diesen Regionen auch der Zugang zu HPV-Impfungen zukünftig eingeschränkt (Kang 2010).

Zusätzlich könnte die HPV-Impfung geschlechtsspezifische Stereotypen fördern, da nur Mädchen und junge Frauen geimpft würden. Folglich enstünde der Eindrück sie alleine wären für reproduktive Gesundheit verantwortlich, obwohl HPV von Männern und Frauen übertragen wird (Kang 2010). Darüber hinaus könnte es aufgrund gesellschaftlicher Bedingungen in Indien für Eltern unangenehm sein, mit ihren Töchtern über die Impfung zu sprechen. Zudem wäre es denkbar, dass Eltern besorgt sind, die Impfung könnte dazu führen, dass sich ihre Töchter beim Geschlechtsverkehr nicht mehr sicher schützen, da sie missverstehen, dass die Impfung nicht gegen alle sexuell übertragbaren Krankheiten schützt (Kang 2010).

Durch die oben aufgeführten Aspekte, die zeigen, dass der Nutzen einer HPV-Impfung für Frauen in Indien bisher noch ungeklärt ist, ergibt sich die Frage, ob HPV-Impfungen tatsächlich das Mittel der Wahl zur Senkung der Krankheitslast von Zervixkarzinomen in Indien darstellen und folglich inwiefern die analysierte Fallstudie mit Blick auf das lokale Interesse von Frauen in Indien initiiert wurde. Hier darf jedoch nicht vergessen werden, dass im Jahre 2020 schätzungsweise 29\% der weltweiten jährlichen 
Zervixkarzinomfälle in Indien auftreten werden und eine Lösung zur Verhinderung neuer Krebsfälle dringend notwendig ist (Shastri 2010).

\subsubsection{Vulnerabilität der Studienteilnehmerinnen in Fallstudie B}

Im folgenden Kapitel soll anhand der in Kapitel 3.4 aufgestellten Vulnerabilitätskriterien (siehe hierzu Abb. 1 Vulnerabilitätskriterien, S. 30) die Frage geklärt werden, ob die Probandinnen der Fallstudie im Kontext ihrer Studienteilnahme eine vulnerable Gruppe darstellen und ihnen somit ein Anrecht auf besonderen Schutz zusteht. Wie bereits im Interpretationsteil der ersten Fallstudie erwähnt, bezieht sich die Überprüfung der Vulnerabilitätskriterien jeweils auf alle Probandinnen der Fallstudie, da keine Informationen über die einzelnen Probandinnen vorliegen und somit eine Einzelfallbetrachtung oder ein Vergleich einer potenziellen Vulnerabilität der Probandinnen untereinander nicht möglich ist. Außerdem beziehen sich auch bei dieser Fallstudie Aussagen über eine mögliche Vulnerabilität der Probandinnen jeweils auf den Kontext ihrer Studienteilnahme und lassen keine Rückschlüsse auf eine darüber hinaus gehende Vulnerabilität zu. Zunächst sollen die Vulnerabilitätskriterien, die zu Kontext der Studie und zu Eigenschaften und Kontext der Person/Personengruppe zählen, überprüft werden. Daraufhin soll am Ende dieses Kapitel die Frage beantwortet werden, ob die Probandinnen eine erhöhte Wahrscheinlichkeit aufweisen, Unrecht zu erfahren und somit ob das Hauptvulnerabilitätskriterium gegeben ist.

\section{A: Kontext der Studie}

\section{Ethisch unzureichende Konzipierung der Studie}

Das erste Vulnerabilitätskriterium, das dem Kontext der Studie zuzuordnen ist, ist die Ethisch un₹ureichende Konzipierung der Studie. Dieses lässt aus mehreren Gründen auf eine mögliche Vulnerabilität der Probandinnen schließen. Durch die Auswahl des Placebos, nämlich Aluminiumhydroxid, das auch im Impfstoff enthalten ist, wurden die Studienteilnehmerinnen unnötigen Risiken ausgesetzt. Die Prüfung der Sicherheit der Impfung verliert durch die Konzipierung der Studie an Aussagekraft, da der Vergleich aufgetretener Nebenwirkungen in der Impfgruppe zur Kontrollgruppe relativ unbrauchbar ist. Um die Sicherheit des Impfstoffes zu testen, hätte man eine Studie mit drei verschiedenen Gruppen, die jeweils entweder den Impfstoff, das Adjuvans (Aluminiumhydroxid) oder eine Kochsalzlösung erhalten, durchführen müssen. Hierdurch hätte man feststellen können, welche Nebenwirkungen durch den Impfstoff und welche möglicherweise durch das Adjuvans hervorgerufen werden. Die Ergebnisse über die Sicherheit und Verträglichkeit des Impfstoffes hätten somit eine höhere Aussagekraft. Bei der Überprüfung der Konzipierung der Studie stellt sich zudem die Frage, warum den Probandinnen keine Vorsorgeuntersuchungen im Zuge der Studienteilnahme angeboten 
wurden. Zum einen hätte man hierdurch eine höhere Aussagekraft über die Wirksamkeit der Impfung durch den Vergleich von aufgetretenen Krebsvorstufen in der Impf- und in der Placebogruppe. Zum anderen hätte man durch das Angebot von kostenlosen Vorsorgeuntersuchungen gewährleisten können, dass die Probandinnen adäquat von der Studienteilnahme profitieren können. Man stelle sich beispielsweise vor, dass einige Probandinnen im Verlauf ihres Lebens an Gebärmutterhalskrebs erkranken, womöglich gar daran versterben könnten, obwohl sie durch ihre Studienteilnahme daran mitgewirkt haben die Krankheitslast von Zervixkarzinomen in Indien zu senken. Somit scheint es aus ethischer Sicht unabdingbar, den Studienteilnehmerinnen Vorsorgeuntersuchungen anzubieten und ihnen hiermit den aktuell üblichen Standard zur Prävention von Zervixkarzinomen nicht vorzuenthalten. Zusätzlich lässt sich mit Blick auf die Ergebnisse der PATRICIA-Studie kritisieren, dass Probandinnen im Alter von 18 bis 35 Jahren rekrutiert wurden, obwohl für diese Altersgruppe eine Wirksamkeit der Studie als äußerst gering einzuschätzen gilt. Allerdings ist nicht nachvollziehbar, ob GSK Biologicals schon während der Laufzeit der Fallstudie Ergebnisse der PATRICA-Studie vorlagen. Ein weiterer zu prüfender Aspekt bezüglich der Konzipierung der Studie ist eine mögliche Ausbeutung der Probandinnen. Hierzu gilt es wie bereits in Kapitel 3.3 erläutert, die Fairness des Nutzens für die Studienteilnahme zu beurteilen. Ein fairer Nutzen wiederum ist abhängig vom NutzenRisiko-Verhältnis und vom Nutzen der beteiligten Pharmafirma im Vergleich zum Nutzen der Probandinnen. Der Nutzen für die Probandinnen durch ihre Studienteilnahme ist als äußerst fragwürdig einzustufen. Es ist beispielsweise immer noch nicht abschließend geklärt, wie wirksam die Impfung tatsächlich zur Verhinderung von Zervixkarzinomen ist und wie hoch somit der medizinische Nutzen für die Probandinnen ist. Des Weiteren hätte das Angebot für regelmäßige Vorsorgeuntersuchungen für die Probandinnen ein sinnvoller Nutzen für eine Studienteilnahme sein können. Für solch ein Angebot gibt es allerdings keine Hinweise. Ein weiterer möglicher Nutzen ist eine angemessene Aufwandsentschädigung, wozu jedoch keine Angaben vorliegen. Zu Risiken beziehungsweise Nachteilen einer Studienteilnahme zählen die Nebenwirkungen, die durch die Impfung oder das Placebo hervorgerufen werden können. Diese wurden bereits im Rahmen der Analyse der Fallstudie detailliert erläutert. Da keine schwerwiegenden unerwünschten Ereignisse, die in Zusammenhang mit dem Impfstoff stehen könnten, auftraten, ist das Risiko für schwerwiegende Nebenwirkungen als gering einzuschätzen. Da der Nutzen für die Probandinnen allerdings ebenfalls als gering einzuschätzen ist und insgesamt die lokale Relevanz einer HPV-Impfung für Frauen in Indien fragwürdig bleibt, ist das Nutzen-Risiko-Verhältnis im Kontext der Studie als unfair einzuschätzen. Der Nutzen der Studiendurchführung für GSK Biologicals ist mit den gegebenen Informationen schwierig einzuschätzen und kann deswegen in die Abwägung eines fairen Nutzens für die Probandinnen nicht miteinbezogen werden. Insgesamt betrachtet lassen die genannten Aspekte den Schluss zu, dass das Vulnerabilitätskriterium Ethisch unzureichende Konæipierung der Studie zutrifft und die Probandinnen im Kontext ihrer Studienteilnahme vulnerabel waren. 


\section{Ethische Ungültigkeit des informed consent}

Hinsichtlich des zweiten Vulnerabilitätskriteriums, das zum Kontext der Studie zählt, nämlich die Ethische Ungültigkeit des informed consent verursacht durch die ForscherInnen, ist es nahezu unmöglich Aussagen zu treffen, da keinerlei Informationen zur Aufklärung vorliegen. Hier wäre es beispielsweise wichtig zu wissen, ob die Aufklärungsformulare in der Muttersprache der jeweiligen Probandin zur Verfügung standen oder ob gegebenenfalls sprachliche Barrieren $\mathrm{zu}$ einem Nichtverstehen studienrelevanter Information geführt haben könnte. In dem Fall müsste der informed consent als ethisch zweifelhaft gelten. Zudem wäre es wichtig zu wissen, ob die Probandinnen ausreichend über mögliche Verhütungsmethoden aufgeklärt wurden und darüber informiert wurden, dass diese kostenfrei durch das Ministry of Health and Family Welfare zur Verfügung stehen. Die Tatsache, dass im Laufe der Studie zehn Schwangerschaften auftraten, obwohl darüber aufgeklärt wurde, dass die Probandinnen während der Studie nicht schwanger werden sollten, lässt dies bezweifeln. Des Weiteren liegen keine Informationen über die Rekrutierung der Probandinnen vor. Hier könnte eine Rekrutierung durch ÄrztInnen, die möglicherweise hierfür eine Provision erhalten, zu Manipulation potenzieller Probandinnen führen und auch hierdurch der informed consent von Seiten des Forscherteams als ethisch unzureichend gelten. Diese Fragen sind jedoch aufgrund der fehlenden Informationen nicht zu beantworten und somit ist unklar, ob das Vulnerabilitätskriterium Ethische Ungültigkeit des informed consent zutreffend ist.

\section{B: Eigenschaften und Kontext der Person/Personengruppe}

\section{Prädisposition, zusätzliches Leid zu erfabren}

Nun sollen die Vulnerabilitätskriterien überprüft werden, die zu Eigenschaften und Kontext der Person/ Personengruppe eingeordnet werden. Das erste diesbezügliche Vulnerabilitätskriterium ist die Prädisposition, zusätzliches Leid zu erfahren. Anhand der gegebenen Informationen kann dies zwar primär verneint, jedoch nicht gänzlich ausgeschlossen werden. Anhand der Einschlusskriterien für die Studienteilnahme, die festlegen, dass nur gesunde Frauen rekrutiert werden sollen, kann davon ausgegangen werden, dass zu Beginn der Studienteilnahme zumindest kein Leid im Sinne einer diagnostizierten physischen oder psychischen Erkrankung vorlag und diesbezüglich somit keine Prädisposition für zusätzliches Leid gegeben ist. Allerdings bezieht sich das Vulnerabilitätskriterium Prädisposition, zusätəliches Leid zu erfahren auch auf eine Form von Leid im weiteren Sinne, so könnten beispielsweise Personen, die in großer Armut leben, anfällig für zusätzliches Leid sein. Inwiefern die Probandinnen in einem weiteren Sinne vorheriges Leid erfahren haben, lässt sich anhand der gegebenen Informationen über die Lebenssituation der Probandinnen nicht ausreichend klären. Auf der Grundlage der wenigen vorhandenen Informationen gibt es keine Hinweise auf zusätzliches Leid durch die Studienteilnahme. Folglich ist dieses Vulnerabilitätskriterium, zumindest mit den vorliegenden Angaben über die Studie und die Probandinnen, nicht gegeben. 


\section{Unfähigkeit informed consent zu erteilen und/oder die Anfälligkeit unfreiwillig quzustimmen}

Das zweite Vulnerabilitätskriterium, das sich auf Eigenschaften und Kontext der Person/Personengruppe bezieht, ist die Unfäbigkeit, informed consent zu erteilen und/oder die Anfälligkeit, unfreiwillig zuzustimmen. Die Unfähigkeit informed consent zu erteilen, kann zum einen durch eine verminderte Kompetenz die Entscheidung für oder gegen eine Studienteilnahme treffen zu können oder durch das Nichtverstehen studienrelevanter Informationen gegeben sein. Ersteres ist im Rahmen dieser Arbeit unmöglich zu beurteilen. Inwiefern alle relevanten Informationen während des informed consent-Prozesses verstanden wurden, ist jedoch insofern zweifelhaft, als dass einige oder möglicherweise sogar viele der Probandinnen Analphabetinnen waren. Die Tatsache, dass eine Probandin Analphabetin ist, lässt nicht automatisch den Schluss zu, dass diese nicht alle studienrelevanten Informationen verstehen kann. Es ist jedoch wesentlich wahrscheinlicher, dass eine Probandin, die nicht lesen und schreiben kann, Informationen, die als Grundlage für eine selbstbestimmte Entscheidung für oder wider eine Studienteilnahme wichtig sind, im Vergleich zu anderen Probandinnen nicht versteht. Es ist nachgewiesen, dass ProbandInnen mit einer höheren Lesekompetenz studienrelevante Informationen signifikant besser verstehen als ProbandInnen mit einer niedrigeren Lesekompetenz (Flory und Emanuel 2004). Zudem gibt es keinerlei Angaben zu besonderen Maßnahmen im Rahmen der Aufklärung der Analphabetinnen, die sicherstellen könnten, dass alle wichtigen Informationen verstanden wurden. Es ist zwar nicht klar, wieviele der Probandinnen der Fallstudie Analphabetinnen waren, aber es sollte davon ausgegangen werden, dass für diese Probandinnen das Vulnerabilitätskriterium Unfähigkeit, informed consent ₹u erteilen und/oder die Anfälligkeit, unfreiwillig zuzustimmen zutreffen könnte. Die Frage, inwiefern im Kontext der Fallstudie die Probandinnen anfällig dafür waren unfreiwillig einer Studienteilnahme zuzustimmen, ist anhand der gegebenen Informationen nicht zu beantworten. Es gibt weder Hinweise auf Zwang oder Manipulation, noch auf einen unangemessenen Anreiz, zum Beispiel im Sinne einer sehr hohen Aufwandsentschädigung oder kostenloser medizinischer Behandlung im Kontext der Studienteilnahme. Letztendlich sollte das Vulnerabilitätskriterium Unfäbigkeit, informed consent zu erteilen und/oder die Anfälligkeit, unfreiwillig zuzustimmen allerdings für die Probandinnen, die Analphabetinnen sind, als zutreffend gewertet werden.

\section{Anfälligkeit, ausgebentet zu werden}

Das dritte Vulnerabilitätskriterium, das zu Eigenschaften und Kontext der Person/Personengruppe zählt, ist die Anfälligkeit, ausgebeutet zu werden. Die Fairness des Nutzens im Rahmen einer Studienteilnahme wurde bereits bezüglich des Vulnerabilitätskriteriums Ethisch un₹ureichende Konzipierung der Studie erläutert. Hierbei stellte sich das Nutzen-Risiko-Verhältnis als vermutlich unfair dar. Da einige der Probandinnen Analphabetinnen waren, könnte dies ein Hinweis darauf sein, dass sich diese möglicherweise in einer prekären sozioökonomischen Situation befanden, da Armut und ein niedriger Bildungsstand häufig einhergehen. Diese Vermutung und die Tatsache, dass die Probandinnen einem eher unfairen Nutzen 
zugestimmt haben, lässt den Schluss zu, dass zumindest einige der Probandinnen im Kontext der Studie anfällig dafür waren ausgebeutet zu werden. Demnach kann dieses Vulnerabilitätskriterium als teilweise zutreffend bezeichnet werden.

Nach der Überprüfung der Vulnerabilitätskriterien bezüglich Kontext der Studie und Eigenschaften und Kontext der Person/Personengruppe lässt sich zusammenfassend festhalten, dass die Vulnerabilitätskriterien Ethisch unzureichende Konzipierung der Studie und Anfälligkeit, ausgebeutet zu werden auf die Probandinnen zutrifft und sie somit im Kontext ihrer Studienteilnahme vulnerabel waren. Zusätzlich gilt für die Probandinnen, die zum Zeitpunkt der Studie Analphabetinnen waren, das Vulnerabilitätskriterium Unfähigkeit, informed consent ₹u erteilen und/oder Anfälligkeit, unfreiwillig zuzustimmen als zutreffend. Hierdurch ergibt sich die Annahme, dass diese Probandinnen ein höheres Maß an Vulnerabilität aufweisen. Daraus folgt, dass für diese Probandinnen im Vergleich $\mathrm{zu}$ den restlichen Probandinnen andere beziehungsweise weitere Schutzmaßnahmen zur Reduzierung der Vulnerabilität notwendig sein könnten. Das Hauptvulnerabilitätskriterium Erböbte Wabrscheinlichkeit, Unrecht zu erfahren trifft allerdings auf alle Probandinnen zu. Ein weiterer Hinweis, der auf eine mögliche Vulnerabilität der Probandinnen schließen lässt, ist die Tatsache, dass von den ForscherInnen keine Angaben zur sozioökonomischen Situation oder zum Bildungsstatus der Probandinnen veröffentlicht wurden. Hierdurch entsteht der Eindruck, dass diesbezügliche Informationen nicht wichtig seien. Zur Beurteilung ethischer Aspekte im Kontext transnationaler Biomedizin und vor allem zur Beurteilung einer möglichen Vulnerabilität der Probandinnen sind diesbezügliche Informationen jedoch von enormer Bedeutung und könnten die Grundlage für die Ausarbeitung sinnvoller Schutzmaßnahmen sein. Fehlende Angaben hierzu lassen somit den Schluss zu, dass die ForscherInnen bei der Durchführung der Studie keine Priorität bei den ethischen Standards setzten. 


\begin{tabular}{|c|c|c|c|c|}
\hline & & \multicolumn{3}{|c|}{ Fallstudie B: HPV-Impfstudie } \\
\hline \multirow{6}{*}{ 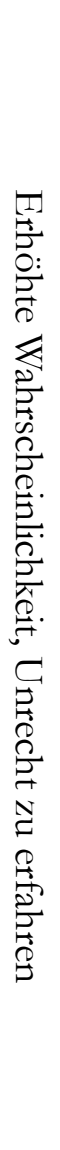 } & & Vulnerabilitätskriterien & Zutreffend? & $\begin{array}{l}\text { zugrundeliegende Aspekte für das (Nicht)- } \\
\text { Zutreffen des Vulnerabilitätsmerkmals }\end{array}$ \\
\hline & \multirow{2}{*}{ 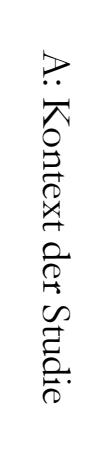 } & $\begin{array}{l}\text { I. Ethisch unzureichende } \\
\text { Konzipierung der Studie }\end{array}$ & ja & $\begin{array}{l}\text { verwendetes Placebo fragwürdig und hierdurch } \\
\text { Aussagen über Sicherheit und Verträglichkeit } \\
\text { der Impfung fragwürdig } \\
\text { - Nutzen für Probandinnen unfair, da z.B. keine } \\
\text { Vorsorgeuntersuchungen angeboten } \\
\text { - lokale Relevanz einer HPV-Impfung für Frauen } \\
\text { in Indien fragwürdig }\end{array}$ \\
\hline & & $\begin{array}{l}\text { II. Ethische Ungültigkeit } \\
\text { des informed consent }\end{array}$ & nein & $\begin{array}{l}\text { - } \quad \text { keine Informationen über Aufklärung und } \\
\text { informed consent-Prozess }\end{array}$ \\
\hline & \multirow{3}{*}{ 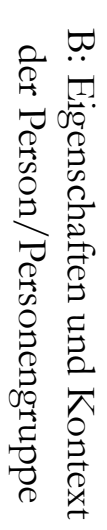 } & $\begin{array}{l}\text { I. Prädisposition, } \\
\text { zusätzliches Leid zu } \\
\text { erfahren }\end{array}$ & nein & $\begin{array}{l}\text { - keine Informationen über vorheriges Leid oder } \\
\text { über zusätzliches Leid durch die } \\
\text { Studienteilnahme }\end{array}$ \\
\hline & & $\begin{array}{l}\text { II. Unfähigkeit, informed } \\
\text { consent zu erteilen und/oder } \\
\text { Anfälligkeit, unfreiwillig } \\
\text { zuzustimmen }\end{array}$ & teilweise & $\begin{array}{l}\text { - zutreffend für Analphabetinnen, da keine } \\
\text { besonderen Schutzmaßnahmen während des } \\
\text { informed consent-Prozesses erwähnt }\end{array}$ \\
\hline & & $\begin{array}{l}\text { III. Anfälligkeit, ausgebeutet } \\
\text { zu werden }\end{array}$ & teilweise & $\begin{array}{l}\text { - möglicherweise zutreffend für Probandinnen } \\
\text { mit niedrigem sozioökonomischen Status } \\
\text { - } \quad \text { Probandinnen stimmten unfairem Nutzen zu }\end{array}$ \\
\hline
\end{tabular}




\subsection{Kurzzusammenfassung und Vergleich der Ergebnisse beider Fallstudien}

Im folgenden Kapitel werden die Ergebnisse der Fallstudien zusammengefasst und miteinander verglichen. Die Tabellen am Ende von Kapitel 5.2.2 (S. 90) beziehungsweise 5.4.2 (S. 113) dienen dabei der Übersichtlichkeit. So lässt sich zusammenfassend festhalten, dass anhand der in Kapitel 3.4 aufgestellten Vulnerabilitätskriterien die jeweiligen Probandinnen beider Studien als vulnerabel gelten müssen. In Fallstudie A (HIV-Impfstudie) ist das Vulnerabilitätskriterium Ethische Ungültigkeit des informed consent, das zum Kontext der Studie zählt, zutreffend. Zusätzlich gilt das Vulnerabilitätskriterium Ethisch unzureichende Konzipierung der Studie, das ebenfalls dem Kontext der Studie zugeordnet wird, als möglicherweise zutreffend. Hinsichtlich der Fallstudie B (HPV-Impfstudie) gilt das zum Kontext der Studie zu verordnende Vulnerabilitätskriterium Ethisch unæureichende Konæipierung der Studie als zutreffend. Des Weiteren ist das zu Eigenschaften und Kontext der Person/Personengruppe zählende Vulnerabilitätskriterium Anfälligkeit, ausgebeutet zu werden teilweise zutreffend. Auf jene Probandinnen der Fallstudie B, die Analphabetinnen waren, trifft zudem das Vulnerabilitätskriterium Unfähigkeit, informed consent zu erteilen und/oder Anfälligkeit, unfreiwillig zuzustimmen zu, das auch $\mathrm{zu}$ Eigenschaften und Kontext der Person/Personengruppe zählt. Beiden Fallstudien ist also gemein, dass jeweils ein Vulnerabilitätskriterium aus dem Bereich Kontext der Studie zutreffend ist. Des Weiteren stellte es sich bei beiden Fallstudien als sehr schwierig heraus das Vulnerabilitätskriterium Prädisposition, zusätzlliches Leid zu erfahren zu überprüfen, da unter anderem jeweils zu wenig Informationen über die tatsächliche Lebenssituation der ProbandInnen angegeben sind.

Es sind aber auch einige Unterschiede hinsichtlich der Vulnerabilität der jeweiligen StudienteilnehmerInnen beider Fallstudien zu nennen. Zunächst fällt bei der Analyse der beiden Fallstudien auf, dass in der Publikation zur HPV-Impfstudie im Gegensatz zur HIV-Impfstudie wesentlich weniger Informationen zur sozioökonomischen Situation oder zum Bildungsstatus der ProbandInnen veröffentlicht wurde. Genau genommen beschränken sich die veröffentlichten Informationen zu den Probandinnen der Fallstudie B auf das Alter der Frauen. Insgesamt werden zur HPV-Impfstudie im Vergleich zur HIV-Impfstudie bedeutend weniger Aspekte beschrieben, die eine Beurteilung des ethischen Standards der Studie zulassen würden, wie beispielsweise der informed consent-Prozess. Die ethischen Standards der Studiendurchführung betreffend liegen diese für die HIV-Impfstudie wesentlich höher im Vergleich zur HPV-Impfstudie. Im Rahmen der HPV-Impfstudie hätte die Vulnerabilität der Probandinnen, die Analphabetinnen waren, durch solche Schutzmaßnahmen, wie sie in der HIV-Impfstudie eingesetzt wurden, möglicherweise reduziert oder gar verhindert werden können. Es muss jedoch darauf hingewiesen werden, dass trotz der hohen ethischen Standards, die im Rahmen der HIV-Impfstudie gesetzt wurden, die ProbandInnen letztendlich anhand der geprüften Kriterien durch Aspekte, die dem Kontext der Studie zuzuordnen sind, als teilweise vulnerabel identifiziert wurden. 


\subsection{Anwendung internationaler Ethikrichtlinien bei der ethischen Bewertung der}

Fallstudien

Im folgenden Kapitel soll beleuchtet werden, inwiefern die in Kapitel 4.1 vorgestellten internationalen Ethikrichtlinien hilfreich für die Analyse und Interpretation beider Fallstudien waren. Die drei wichtigsten internationalen Ethikrichtlinien wurden in Kapitel 4.1 bezüglich ihrer Herangehensweisen an das Konzept der Vulnerabilität, an ethische Konflikte im Rahmen medizinischer Forschung mit Frauen und hinsichtlich transnationaler Biomedizin dargestellt. Für die Analyse der Fallstudien stellten sich die International Ethical Guidelines for Biomedical Research Involving Human Subjects der CIOMS als besonders hilfreich heraus. Die meisten der darin enthaltenen Punkte stellen explizite Forderungen für den Kontext transnationaler Biomedizin auf. Die Deklaration von Helsinki hingegen thematisiert Aspekte, die transnationale Biomedizin betreffen, nur indirekt und zudem wenig detailliert. Die Universal Declaration on Bioethics and Human Rights der UNESCO ist insofern eine fruchtbare Ergänzung, da diese ihre Forderungen hauptsächlich an Staaten adressiert und Gesetzgebungen zum Schutz von ProbandInnen in Entwicklungsländern eine bedeutende Rolle spielen. Dies zeigt sich beispielsweise anhand der Gesetzesänderungen, die Anfang des Jahres 2013 in Indien vorgenommen wurden (siehe hierzu Kapitel 4.2.1).

Ein großes Problem, alle drei Richtlinien betreffend, ist die Tatsache, dass diese sogenanntes soft law darstellen. Wie bereits in Kapitel 4.1 erläutert, bedeutet dies, dass die Umsetzung der Forderungen nicht rechtlich bindend für ForscherInnen und SponsorInnen ist. Bei der Durchführung der Studien, die für diese Arbeit als Fallstudien dienten, hätten jedoch bei der exakten Einhaltung aller drei Ethikrichtlinien einige ethische Probleme verhindert werden können. Die ungenügende Aufklärung der ProbandInnen über die Forschungsergebnisse aus Europa bei Fallstudie A, stellen beispielsweise einen Verstoß gegen Punkt 6 in den Guidelines der CIOMS dar (siehe hierzu Kapitel 5.2.2). Darüber hinaus ist die fragwürdige Wahl des Placebos in Fallstudie B (siehe hierzu Kapitel 5.4.1), nämlich ein Adjuvans, das auch in der HPV-Impfung selbst enthalten ist, beispielsweise eine Verletzung des Paragraphen 33 der Deklaration von Helsinki. Dieser besagt unter anderem, dass ein Placebo nur verwendet werden darf, wenn es aus wissenschaftlichen Gründen zwingend notwendig sei und wenn die ProbandInnen keinem erhöhten Risiko Schaden zu erleiden, ausgesetzt würden. Sowohl aus wissenschaftlicher als auch aus ethischer Sicht ist die Auswahl des Placebos in Fallstudie B allerdings als nicht sinnvoll zu werten (siehe hierzu Kapitel 5.4.1 und 5.4.2).

Zusätzlich muss an dieser Stelle nochmals auf die unterschiedlichen Herangehensweisen an das Konzept der Vulnerabilität innerhalb der Richtlinien eingegangen werden. Die Änderungen der Deklaration von Helsinki (2013) bezüglich Vulnerabilität, wie bereits in Kapitel 4.1.1 erörtert, sind sehr begrüßenswert. Zuvor wurde das Verständnis von Vulnerabilität ausschließlich mit informed consent verknüpft (Deklaration von Helsinki aus dem Jahre 2008). 
Die Ergebnisse der Interpretation der Fallstudien und auch die Bedeutung der drei vorgestellten internationalen Ethikrichtlinien für die ethische Bewertung der Fallstudien sollen für die abschließende Diskussion in Kapitel 6 im Blick behalten werden. 


\section{Diskussion und Fazit}

Im folgenden Kapitel wird die ethische Bedeutung der Ergebnisse abschließend diskutiert. Hierbei sollen sowohl das theoretische Konzept der Vulnerabilität als auch dessen mögliche praktische Umsetzung für die Beurteilung ethischer Aspekte im Kontext medizinischer Forschung erörtert und Ansätze zur Weiterentwicklung aufgezeigt werden.

\section{$\underline{\text { 6.1 Theoretische Implikationen für das Vulnerabilitätskonzept }}$}

Zunächst gilt es, die Anwendung der erarbeiteten Vulnerabilitätskriterien und mögliche Schwierigkeiten hierbei aus theoretischer Sicht $\mathrm{zu}$ beleuchten (Kapitel 6.1.1). Um die Universalität der Vulnerabilitätskriterien zu prüfen, sollen diese im Anschluss mit ethischen Kriterien verglichen werden, die eine grundsätzliche Vulnerabilität von Kindern rechtfertigen (Kapitel 6.1.2). Daraufhin sollen in diesem Kapitel allgemeine Einschränkungen bei der Anwendung des Konzepts der Vulnerabilität diskutiert werden. Ergänzend hierzu soll vor dem Hintergrund dieser Arbeit die Notwendigkeit des Vulnerabilitätskonzepts für die Bioethik untersucht werden (Kapitel 6.1.3).

\subsubsection{Bedeutung der Ergebnisse für die Anwendung der erarbeiteten} Vulnerabilitätskriterien

$\mathrm{Zu}$ Beginn dieses Kapitels soll darauf hingewiesen werden, dass bei beiden Fallstudien Vulnerabilitätskriterien, die zum Kontext der Studie zählen, als zutreffend identifiziert wurden. Dies stützt die These von Hurst (2008), dass für die Beurteilung einer potenziellen Vulnerabilität von ProbandInnen, die konkreten Gegebenheiten einer Studie mehr in den Fokus der Analyse gerückt werden müssten (siehe hierzu Kapitel 3.1). Vor allem anhand der Durchführung von Fallstudie A (HIVImpfstudie) zeigt sich, dass der Kontext der Studie mehr berücksichtigt werden sollte. Bei dieser Studie wurde versucht, hohe ethische Standards zu erfüllen. Um dies zu erreichen, wurde seitens der ForscherInnen das Hauptaugenmerk auf den informed consent gerichtet. Dies wird beispielsweise durch die ausführliche Beschreibung des informed consent-Prozesses und den eigens für die Studie entwickelten Verständnistest deutlich. Aufgrund dieser Schutzmaßnahmen, kann angenommen werden, dass die ForscherInnen der Fallstudie A davon ausgingen, dass einige der potenziellen ProbandInnen vulnerabel sein könnten und deren Vulnerabilität im Sinne einer Unfähigkeit informed consent zu erteilen, bestehe. Es ist sehr wahrscheinlich, dass ohne diese Schutzmaßnahmen ProbandInnen an Fallstudie A 
teilgenommen hätten, welche aufgrund einer Unfähigkeit informed consent zu geben, vulnerabel gewesen wären. Folglich sollten die getroffenen Schutzmaßnahmen als notwendig gelten und positiv bewertet werden. Anhand der Interpretation der Fallstudie ließ sich allerdings feststellen, dass die ProbandInnen trotz der Schutzmaßnahmen vulnerabel waren und dies anhand des weiteren Vulnerabilitätskriteriums Ethische Ungültigkeit des informed consent, das zum Kontext der Studie zählt. Folglich ergibt sich die Notwendigkeit, den Kontext einer Studie bei Beurteilung einer möglichen Vulnerabilität von ProbandInnen ebenso zu berücksichtigen wie Aspekte, die die Personen selbst betreffen, also beispielsweise die Unfähigkeit einen informed consent zu erteilen. Hierdurch zeigt sich, dass die in dieser Arbeit vorgenommene theoretische Kombination aus kontextbezogenen und personenbezogenen Aspekten praktisch sinnvoll ist. Zudem wird deutlich, dass eine Erweiterung der Vulnerabilitätskriterien über den informed consent hinaus notwendig bleibt. So war bei beiden Fallstudien zum Beispiel die Prüfung des Vulnerabilitätskriteriums Ethisch unzureichende Kon₹ipierung der Studie äußerst aufschlussreich. Im Hinblick auf Fallstudie B und die Teilnahme von Analphabetinnen wird zudem deutlich, dass es sinnvoll ist, Vulnerabilität als ein graduelles Konzept und nicht nur in binärer Konstellation zu verstehen (vulnerabel versus nicht vulnerabel). Hiermit ist gemeint, dass manche Personen im Vergleich zu anderen und je nach Studienkontext und auch je nach aktueller Lebenssituation mehr oder weniger vulnerabel sein können. ProbandInnen innerhalb derselben Studie können auch unterschiedliche Grade an Vulnerabilität aufweisen. Bei Fallstudie B gelten zwar alle Probandinnen als vulnerabel, Analphabetinnen weisen jedoch ein höheres Maß an Vulnerabilität auf als Nicht-Analphabetinnen. Denn für sie trifft zusätzlich das Vulnerabilitätskriterium Unfäbigkeit, informed consent zu erteilen und/oder Anfälligkeit, unfreiwillig zurustimmen zu.

Die Verwendung mehrerer, unterschiedlicher Vulnerabilitätskriterien lässt die Einschätzung verschiedener Grade von Vulnerabilität zu. Treffen beispielsweise drei Vulnerabilitätskriterien auf eine bestimmte ProbandInnengruppe zu, ist von einer höhergradigen Vulnerabilität auszugehen im Vergleich zu einer ProbandInnengruppe, für die nur ein Vulnerabilitätskriterium zutrifft. Zusätzlich können die Gründe, die für das Zutreffen eines Vulnerabilitätskriteriums sprechen, Hinweise für eine hochgradige oder weniger ausgeprägte Form von Vulnerabilität sein. Hiermit ist gemeint, dass es beispielsweise nicht unerheblich ist, wie viele Gründe für das Auftreten eines Vulnerabilitätskriteriums angegeben werden können. Auf unterschiedliche Grade von Vulnerabilität sollte zudem mit unterschiedlichen Schutzmaßnahmen reagiert werden, um sicherzustellen, dass die betreffenden Personen angemessenen Schutz erhalten.

Als grundsätzlich schwierig anzuwenden muss hingegen die Überprüfung des Vulnerabilitätskriteriums Prädisposition, zusätəliches Leid zu erfahren gelten. Dies haben zumindest die beiden unterschiedlichen Fallstudien ergeben. Es bleibt zwar einleuchtend, dass Personen, die bereits Leid erfahren haben, anfällig für zusätzliches Leid sind (Kottow 2003). Demnach benötigen diese Personen besonderen Schutz, sollten also als vulnerabel gelten (siehe hierzu Kapitel 3.1). Der Begriff Leid ist allerdings 
schwierig zu fassen. Zum einen ist in dieser Arbeit physisches und psychisches Leid gemeint. Zum anderen soll das Vulnerabilitätskriteriums Prädisposition, zusätz̧liches Leid zu erfahren auch Leid in einem weiteren Sinne, wie beispielsweise aufgrund einer prekären finanziellen Lage oder Machtlosigkeit beinhalten. Zur Beurteilung, ob eine Anfälligkeit für zusätzliches Leid besteht, müsste allerdings zunächst geklärt werden, ob vorheriges Leid besteht (Hurst 2008). Bei der Interpretation der Fallstudien konnte allerdings nur bis zu einem gewissen Grad ausgeschlossen werden, dass vorheriges physisches oder psychisches Leid vorlag. So stellte zwar die Gesundheit der ProbandInnen jeweils ein Einschlusskriterium für die Studienteilnahme dar (beziehungsweise Krankheit ein Ausschlusskriterium). Es ist aber auch denkbar, dass bei manchen ProbandInnen möglicherweise eine nicht offensichtliche oder bisher nicht diagnostizierte Erkrankung vorgelegen haben könnte. Darüber hinaus bleibt die Frage offen, ob zusätzliches Leid durch die Studienteilnahme aufgetreten ist. Zusätzliches Leid im Sinne von physischem und eventuell auch psychischem Leid könnte zum Teil anhand der aufgetretenen Nebenwirkungen und unerwünschten Ereignisse, die mit dem getesteten Medikament als in Verbindung stehend eingeschätzt wurden, beurteilt werden. Jedoch bleibt auch bei der retrospektiven Überprüfung einer Studie die Bewertung von zusätzlichem Leid in einem weiteren Sinne aus, da nicht bewertet werden kann, ob ProbandInnen beispielsweise durch eine prekäre sozioökonomische Lage Leid erfahren.

Trotz der Schwierigkeit das Vulnerabilitätskriterium Prädisposition, zusätəliches Leid zu erfahren zu überprüfen, soll dieses jedoch für die Beurteilung einer möglichen Vulnerabilität von Frauen im Kontext transnationaler Biomedizin beibehalten werden. Dies wird damit begründet, dass besonders bei diesem Vulnerabilitätskriterium die betroffenen Personen, zum Beispiel potenzielle Probandinnen, selbst bestimmen könnten, ob ihnen durch eine Studienteilnahme zusätzliches Leid im physischem, psychischen oder auch sozialem Sinne widerfahren könnte oder widerfahren ist. Hierdurch kann aufgezeigt werden, dass die Perspektive der betroffenen Personen bei der Bewertung von Vulnerabilität wichtig ist. Nur diese können schlussendlich festlegen, was für sie Leid bedeutet und ob ihnen Leid widerfahren ist. Folglich ließe sich mit dem Einsatz des Vulnerabilitätskriteriums Prädisposition, zusätzliches Leid zu erfahren die Problematik adressieren, dass nicht-betroffene Personen beurteilen, ob andere Personen vulnerabel sind. Bislang blieb das Mitspracherecht der betroffenen Personen selbst aus. Die Mitbestimmung von ProbandInnen bei der Prüfung einer möglichen Vulnerabilität im Kontext einer Studienteilnahme und bei der darauf folgenden Festlegung von adäquaten Schutzmaßnahmen könnte jedoch gerade mithilfe des Leidbegriffs bestärkt werden. Demnach soll dieses Vulnerabilitätskriterium beibehalten werden.

Dies gilt ähnlich auch für das Vulnerabilitätskriterium Ethisch un₹ureichende Konzipierung der Studie. Dieses beinhaltet beispielsweise die Beurteilung eines fairen Nutzens für die Studienteilnahme und demnach die Beurteilung, ob ProbandInnen im Rahmen ihrer Studienteilnahme ausgebeutet wurden. Hierbei besteht die Gefahr, dass man bestimmte potenzielle Nutzen und Risiken unter- oder überschätzt. 
Demnach könnte in Grenzfällen, je nach dem wer das Vulnerabilitätskriterium überprüft, der Nutzen als fair oder unfair eingeschätzt werden. Bei der Beurteilung, ob der Nutzen für eine Studienteilnahme fair ist, sollte daher den betreffenden ProbandInnen ein Mitsprachrecht eingeräumt werden. Das vorgelegte Konzept der Vulnerabilität bietet somit die Chance sich konkret für die Stärkung der Autonomie und Selbstbestimmung von ProbandInnen einzusetzen, da diese selbst definieren könnten, ob sie im Rahmen ihrer Studie vulnerabel sind oder nicht. Zudem ist es sinnvoll die betroffenen ProbandInnen bei der Erarbeitung möglicher Schutzmaßnahmen und der Überprüfung, ob diese angemessen sind, direkt miteinzubeziehen.

Zusammenfassend bietet das Konzept der Vulnerabilität die Möglichkeit, die Sichtweise betroffener Personen miteinzubeziehen und ethische Aspekte bezüglich Forschung mit diesen Personen nicht gänzlich von außen zu beurteilen.

Darüber hinaus ergab sich bei der notwendigen Prüfung der Vulnerabilitätskriterien die Schwierigkeit, dass deren Beurteilung auch im Wesentlichen davon abhängt, ob ausreichend Informationen über die ProbandInnen, deren Lebenssituation, den informed consent-Prozess und die Konzipierung der Studie vorliegen. Für eine sinnvolle Überprüfung der Vulnerabilitätskriterien ist es folglich notwendig, dass die ForscherInnen und SponsorInnen die erforderlichen Informationen bereitstellen. Insbesondere die Analyse von Fallstudie B zeigt, dass viele Informationen in der Veröffentlichung zur Studie fehlten, um die Vulnerabilitätskriterien adäquat prüfen zu können. Daher muss an dieser Stelle nochmals betont werden, dass solch eine ethische Überprüfung nicht als einmaliger Prozess, beispielsweise durch ein bestimmtes Ethikkommission, geschehen kann, sondern aufgrund kultureller und theoretischer Differenzen dem Diskurs bzw. der Fachcommunity im weiteren Sinn auch möglich sein muss. Demnach folgt die Forderung nach mehr Information und Transparenz darüber, mit wem unter welchen Umständen geforscht wird.

Ein möglicher Kritikpunkt an der Vorgehensweise dieser Arbeit, ist die Tatsache, dass nur zwei Fallstudien bearbeitet wurden. Hierdurch ist die Aussagekraft bezüglich der Vulnerabilität von Frauen in Indien im Kontext transnationaler Biomedizin begrenzt. Weitere Fallstudien zu analysieren ist allerdings im Rahmen dieser Arbeit nicht möglich. Die Arbeit bietet jedoch eine Grundlage auf der weitere Fallstudien anhand der erarbeiteten Vulnerabilitätskriterien analysiert werden können. Darüber hinaus gilt es als kritisch zu beurteilen, dass die ProbandInnen der Fallstudien anhand der Analyse und Interpretation als vulnerabel im Kontext ihrer Studienteilnahme bewertet wurden, die ProbandInnen selbst hierzu jedoch nicht befragt wurden. Dies ist zusätzlich Anhalt für zukünftige empirische Forschung auf diesem Gebiet (siehe hierzu Kapitel 6.3). 


\subsubsection{Vulnerabilität im Kontext transnationaler Biomedizin im Vergleich mit}

Vulnerabilität von Kindern

Die spezifischen Vulnerabilitätskriterien für einen bestimmten Kontext, nämlich Forschung mit Frauen im Rahmen transnationaler Biomedizin, wurden aufgestellt, um eine in der internationalen Forschung wachsende Tendenz besser bewerten zu können. Es muss jedoch nochmal die Frage gestellt werden, ob es überhaupt sinnvoll ist, spezifische Vulnerabilitätskriterien für bestimmte Personen oder Personengruppen aufzustellen. Hierfür soll überprüft werden, inwiefern sich die erarbeiteten Vulnerabilitätskriterien von Aspekten unterscheiden, die Vulnerabilität im Rahmen von Forschung mit Kindern betreffen. Ein diesbezüglicher Vergleich ist insofern fruchtbar, als Kinder anerkannterweise als vulnerable Gruppe gelten und bei der Aufzählung vulnerabler Gruppen sehr häufig als Paradebeispiel genannt werden (Kopelman 2004, S. 2365; CIOMS 2002, S. 64). Die Vulnerabilität von Kindern wird häufig mit dem informed consent verknüpft. Als Gründe für die Vulnerabilität von Kindern wird beispielsweise die eingeschränkte Fähigkeit zu verstehen und die Anfälligkeit unter Zwang zuzustimmen, aufgeführt (Davidson und O'Brien 2009). Beide Aspekte führen letztlich zu einem ethisch unzureichenden informed consent. Die Fähigkeit zu verstehen und die Fähigkeit, informed consent zu erteilen, hängen allerdings vom Alter und von der Reife eines Kindes ab (Davidson und O'Brien 2009). Demnach zeigt sich, dass bei der Erarbeitung von Vulnerabilitätskriterien von Kindern als potenzielle ProbandInnen Aspekte, wie die Unfähigkeit informed consent zu erteilen, die Anfälligkeit unfreiwillig zuzustimmen und das Alter und die Reife eines Kindes eine große Rolle spielen müssten. Einerseits deckt sich dies teilweise mit den hier verwendeten Vulnerabilitätskriterien, wie beispielsweise die Unfäbigkeit, informed consent zu erteilen und/oder die Anfälligkeit, unfreiwillig zu₹ustimmen. Andererseits werden Alter und mentale Reife von ProbandInnen in den Vulnerabilitätskriterien für Frauen im Kontext transnationaler Biomedizin nicht berücksichtigt, sie sind jedoch für die Beurteilung von Vulnerabilität von Kindern von großer Bedeutung. Abschließend lässt sich festhalten, dass zumindest bezüglich der Forschung mit Kindern, die üblicherweise als vulnerabel gelten, andere Schwerpunkte bei der Überprüfung von Vulnerabilität gelegt werden müssten als im Kontext dieser Arbeit.

Man könnte jedoch argumentieren, dass die Operationalisierung des Konzepts der Vulnerabilität universal für alle vulnerablen Personen oder Personengruppen gültig sein müsste, wie dies beispielsweise Hurst (2008) versucht. Zugegeben scheint es möglicherweise als zu aufwändig und unpraktikabel, für jegliche Personengruppen, die vulnerabel sein könnten, eigene Vulnerabilitätskriterien aufzustellen und diese dann je nach Kontext erneut zu überprüfen. Ein guter Grund, dies dennoch zu tun, ist, dass hierin die Chance besteht, dass sich BioethikerInnen und ForscherInnen ausführlich mit bestimmten ProbandInnengruppen auseinandersetzen würden, die potenziell vulnerabel sein könnten. Hierdurch würde man eine stärkere Sensibilisierung für bestimmte Personen oder Personengruppen und für spezifische ethische Aspekte, die Forschung mit diesen Personen betrifft, 
erreichen. Des Weiteren erscheint es nahezu unmöglich, universale Vulnerabilitätskriterien, die für alle vulnerablen Personen und Personengruppen zutreffen, aufzustellen, die nicht zu weit und nicht zu eng gefasst und trotzdem konkret genug sind, um eine mögliche Vulnerabilität von bestimmten Personen oder Personengruppen überprüfen zu können. Diese Schwierigkeit zeigt sich genau in der Tatsache, dass bisher keine einheitliche Definition von Vulnerabilität in den wichtigen Richtlinien existiert. Somit gibt es auch keine einheitliche Vorgehensweise bei der Operationalisierung des Konzepts.

Es soll allerdings darauf hingewiesen werden, dass das in dieser Arbeit verwendete Hauptvulnerabilitätskriterium, von Hursts Ansatz stammend, universal für alle vulnerablen Personen und Personengruppen zutrifft, nämlich die erböbte Wabrscheinlichkeit, Unrecht zu erfahren. Dieses Vulnerabilitätskriterium ist weder zu weit noch zu eng gefasst, da es kontextabhängig verwendet wird. Demnach sind nicht einfach bestimmte Gruppen vulnerabel, sondern Personen oder Personengruppen weisen in bestimmten Kontexten eine erhöhte Wahrscheinlichkeit auf Unrecht zu erfahren und sind dann vulnerabel (siehe hierzu Kapitel 3.1). Um vulnerable Personengruppen jedoch tatsächlich identifizieren zu können, ist dieses Vulnerabilitätskriterium zu abstrakt, da unklar ist, wer und anhand welcher Kriterien die erhöhte Wahrscheinlichkeit, Unrecht zu erfahren geprüft würde.

Hieraus ergibt sich also die Notwendigkeit der Formulierung spezifischer Vulnerabilitätskriterien für die Identifizierung potenziell vulnerabler Gruppen, wie beispielsweise für Kinder, psychisch kranke oder demente Personen. Hieraus erklärt sich also die Vorgehensweise dieser Arbeit. Es wurden anhand ethischer Konflikte spezifische Vulnerabilitätskriterien erarbeitet, die insbesondere im Kontext transnationaler Forschung mit Frauen von Bedeutung sind und bei Anwendung verschiedene Grade von Vulnerabilität sichtbar machen.

\subsubsection{Kritik an der Praktikabilität und Notwendigkeit des Vulnerabilitätskonzepts}

Zur normativen Bewertung der Ergebnisse dieser Arbeit folgt in diesem Kapitel die Diskussion allgemeiner Kritikpunkte und Einwände am Konzept der Vulnerabilität und dessen Anwendung in der Bioethik. Einige Kritikpunkte wurden bereits in Kapitel 3.1 erläutert (Levine et al. 2004). Hier wird darauf eingegangen, inwiefern diese Kritikpunkte im Rahmen der vorliegenden Analyse der Fallstudien aufgegriffen wurden.

Zuerst wurde von Levine et al. kritisiert, dass zu viele Personen und Personengruppen als vulnerabel bezeichnet werden und somit fast jeder als vulnerabel gilt, wodurch das Konzept zu weit gefasst sei und an Aussagekraft verlöre (Levine et al. 2004). Der zweite Kritikpunkt war, dass das Konzept der Vulnerabilität wiederum häufig zu eng gefasst sei, da nur Eigenschaften von bestimmten Personengruppen im Fokus stünden (Levine et al. 2004). Beide Kritikpunkte wurden in dieser Arbeit insofern aufgegriffen, als dass bei der Analyse der Fallstudien die Kontextabhängigkeit der 
Vulnerabilität der ProbandInnen betont wurde. Dies bedeutet, dass die festgestellte Vulnerabilität der ProbandInnen mithilfe der Vulnerabilitätskriterien Aussagen über die jeweils untersuchte Fallstudie zulässt, nicht jedoch darüber hinaus. Somit wurde gewährleistet, dass das Konzept der Vulnerabilität nur für den Forschungskontext gilt und keine Aussagen über die Personen im Allgemeinen macht. Hiermit wurden soziale Stereotypen, wie beispielsweise ein Deklarieren aller Frauen in Entwicklungsländern als vulnerabel, vermieden. Durch das Einbeziehen des Kontextes der Studie als ein Teil der Vulnerabilitätskriterien, den Eigenschaften und der Lebenssituation der ProbandInnen gleichwertig gegenüberstehend, wurde auch verhindert, dass nur die Eigenschaften von Personen über vulnerabel oder nicht vulnerabel entscheiden.

Als ein dritter Punkt, wurde die Kategorisierung bestimmter Personengruppen per se als vulnerabel kritisiert, wie beispielsweise Schwangere, da dies den Nachteil der Stereotypisierung mit sich brächte (Levine et al. 2004). Hierbei würden Personen, die Teil einer bestimmten Gruppe sind, fälschlicherweise als vulnerabel bezeichnet. Hinsichtlich der analysierten Fallstudien ist diese Problematik nicht zu unterschätzen. Im Zuge der Interpretation der Fallstudie B (HPV-Impfstudie) wurde nämlich das Vulnerabilitätskriterium Unfähigkeit, informed consent zu erteilen und/oder Anfälligkeit, unfreiwillig zuzustimmen als eher zutreffend für Analphabetinnen gewertet. Dies kann durchaus als Stereotypisierung verstanden werden. Man könnte einwenden, dass die Tatsache, dass eine Frau nicht lesen und schreiben kann nicht gleichzeitig bedeutet, dass diese unfähig ist informed consent für eine Studienteilnahme zu erteilen. Dem wäre zu entgegnen, dass in der Publikation zu Fallstudie B weder Angaben dazu gemacht wurden, dass Analphabetinnen an der Studie teilnahmen, noch inwiefern es besondere Maßnahmen im Rahmen des informed consent-Prozesses für diese Probandinnen gab. Die Information über die Teilnahme von Analphabetinnen an der Studie wurde erst auf gezieltes Nachfragen hinsichtlich des Bildungsstatus der Probandinnen von der Erstautorin offengelegt. ${ }^{15}$ Hierdurch zeigt sich, dass der Aspekt Analphabetismus für das Forscherteam vermutlich nicht von großer Relevanz war, sonst hätte man dies in der Publikation erwähnt. Daher kann davon ausgegangen werden, dass für diese Probandinnen keine besonderen Maßnahmen getroffen wurden, um sicherzustellen, dass diese vor der Einwilligung alle relevanten Informationen verstanden haben. Dies ist ein Hinweis dafür, dass die ForscherInnen der Fallstudie B keine Priorität beim Schutz ihrer Probandinnen setzten. Zudem geht ein niedriger Bildungsstatus häufig mit einer schwierigen finanziellen Lage einher (Denny und Grady 2006). Dies stützt die Annahme, dass diese Probandinnen diesbezüglich vulnerabel sein könnten und besonderen Schutz benötigen. Letzteres und die zuvor genannten Annahmen über die Prioritäten der ForscherInnen lassen den Schluss zu, dass für die Analphabetinnen der Fallstudie B das Vulnerabilitätskriterium Unfähigkeit, informed consent zu erteilen und/oder Anfälligkeit, unfreiwillig zuzustimmen plausibel ist und es sich hierbei nicht um eine genrelle Stereotypisierung von Analphabetinnen handelt.

\footnotetext{
${ }^{15}$ Persönliche Kommunikation mit Erstautorin (Email vom 15.09.2012)
} 
Abschließend soll noch der mögliche Einwand aufgegriffen werden, dass das Konzept der Vulnerabilität für bioethische Diskurse und die Beurteilung ethischer Aspekte im Kontext medizinischer Forschung nicht notwendig sei. Betrachtet man das Konzept des informed consent, scheint es zunächst plausibel anzunehmen, dass dies ausreicht, um potenzielle ProbandInnen im Kontext medizinischer Forschung vor zusätzlichem oder erheblichen Leid und/oder Unrecht zu schützen. Stelle man sich beispielsweise eine Person vor, die kompetent ist, ausreichend aufgeklärt wird, alle relevanten Informationen versteht und einer Studienteilnahme aus freien Stücken zustimmt, dann ist es schwer vorstellbar, dass dieser Person mit einer erhöhten Wahrscheinlichkeit Unrecht widerfahren könnte. Hier liegt die Annahme zugrunde, dass ein ethisch ausreichender informed consent, wie in Kapitel 3.1 erläutert, der die Elemente Kompetenz, Freiwilligkeit, Aufklärung, Verstehen, Entscheidungsfindung und Zustimmung beziehungsweise Ablehnung mitberücksichtigt, ausreichenden Schutz gewährleistet. Es können jedoch mindestens drei gute Gründe aus dieser Arbeit abgeleitet werden, die dieser Annahme widersprechen.

Erstens wurde nämlich bereits in Kapitel 3.3 gezeigt, dass Ausbeutung nicht mithilfe eines ethisch ausreichenden informed consent verhindert werden kann. Dies zeigt sich dadurch, dass eine Person freiwillig und selbstbestimmt einer ausbeutenden Transaktion zustimmen könnte, da dies womöglich für diese Person in ihrer aktuellen Situation die beste Option darstellt. Hier kann mithilfe des Vulnerabilitätskonzepts sowohl Ausbeutung im Rahmen einer Studie als auch die Anfälligkeit bestimmter Personen, ausgebeutet zu werden, identifiziert werden. Daraufhin können entsprechende Schutzmaßnahmen veranlasst werden. Die Anwendung des Konzepts der Vulnerabilität hinsichtlich der Verhinderung von Ausbeutung, könnte darin bestehen, mithilfe des Vulnerabilitätskriteriums ethisch un₹ureichende Kon₹ipierung der Studie zu überprüfen, ob die ProbandInnen einen fairen Nutzen für ihre Studienteilnahme erhalten. Zusätzlich kann anhand des Vulnerabilitätskriteriums Anfälligkeit, ausgebeutet zu werden identifiziert werden, welche potenziellen ProbandInnen aufgrund ihrer aktuellen Lebenssituation anfällig dafür sind, einem unfairen Nutzen zuzustimmen. Eine mögliche Schutzmaßnahme diesbezüglich ist die Gewährleistung eines fairen Nutzens. Die Notwendigkeit, das Konzept der Vulnerabilität zu verwenden, um auch Ausbeutung und somit Unrecht aufzeigen zu können, wurde bereits anhand der beiden Fallstudien deutlich. Bei Fallstudie B gilt nämlich, unter anderem wegen der Bewertung des Nutzens als unfair, das Vulnerabilitätskriterium Ethisch un₹ureichende Konzipierung der Studie als zutreffend. Letztlich kann also davon ausgegangen werden, dass diese Probandinnen im Rahmen ihrer Studienteilnahme ausgebeutet wurden. Darüber hinaus wurde das Vulnerabilitätskriterium Anfälligkeit, ausgebeutet zu werden als teilweise zutreffend interpretiert. Dies bedeutet, dass ethische Probleme zumindest im Kontext transnationaler Biomedizin nicht gänzlich mit einem ethisch ausreichenden informed consent gelöst werden können, sondern das Konzept der Vulnerabilität hier eine notwendige und sinnvolle Anwendung findet. Außerdem kann das Konzept der Vulnerabilität Aspekte, die den informed consent betreffen, wie beispielsweise das Vulnerabilitätskriterium 
Unfähigkeit, informed consent zu erteilen und/oder Anfälligkeit, unfreiwillig zuzustimmen ausdifferenzieren. Folglich schließt das Konzept der Vulnerabilität einen ethisch gültigen informed consent mit ein, bietet jedoch darüber hinaus Möglichkeiten, ethische Aspekte im Rahmen medizinischer Forschung zu beleuchten und daraus hervorgehende normative Forderungen zu stellen, die über die Anwendung des informed consent hinausgehen. Demnach können mit dem Konzept der Vulnerabilität mehrere Kriterien kombiniert werden.

Ein zweiter Grund für die Etablierung des Vulnerabilitätskonzepts in der Bioethik ist, dass es die Möglichkeit bietet, stärker für bestimmte Personen und Personengruppen zu sensibilisieren. Hiermit ist gemeint, dass man im Rahmen einer Überprüfung, ob Personen in einem konkreten Kontext vulnerabel sind, mögliche ethische Konflikte bei Forschung mit diesen Personen beleuchtet und hierdurch möglicherweise Aspekte identifiziert, die zuvor unbeachtet blieben. Demnach sollte man die Vulnerabilität bestimmter Personengruppen, die mit einer erhöhten Wahrscheinlichkeit vulnerabel sein könnten, wie beispielsweise Kinder oder Frauen in Entwicklungsländern, strenger prüfen.

Allerdings soll an dieser Stelle nochmals betont werden, dass die Anwendung eines sinnvollen Konzepts der Vulnerabilität nicht darin besteht, Personengruppen als per se vulnerabel zu kategorisieren und damit von medizinischer Forschung prinzipiell auszuschließen. Eine formale Liste von vulnerablen Personengruppen aufstellen zu wollen, würde zu Stereotypisierung und vermutlich auch zur Missachtung der Autonomie von StudienteilnehmerInnen führen. Deswegen soll hier der Ansatz von Hurst hervorgehoben werden und Vulnerabilität kontextualisiert verwendet und je nach Studie entlang der aufgestellten Vulnerabilitätskriterien überprüft werden, ob bestimmte Personen oder Personengruppen im Rahmen ihrer Studienteilnahme vulnerabel sein könnten (Hurst 2008).

Drittens wird erst durch das Konzept der Vulnerabilität deutlich, dass noch Forderungen nach stärkerer Transparenz im Zuge medizinischer Forschung beziehungsweise ihrer Publikationen notwendig sind. Erst das Offenlegen ausführlicher Informationen sowohl über die StudienteilnehmerInnen und deren Lebenssituation als auch über den Nutzen einer Studienteilnahme macht es möglich, Vulnerabilitätskriterien zu überprüfen. Entsprechend sollten auch Informationen über den Umgang und die Maßnahmen zur Reduzierung einer potenziellen Vulnerabilität von ProbandInnen transparent gestaltet werden. Vor allem durch Fallstudie B zeigte sich nämlich, dass die ausschließliche Bewertung durch ein bestimmtes Ethikkomitee beziehungsweise die Angabe in der Publikation, dass ein Ethikvotum vorhanden sei, nicht ausreicht, um Vulnerabilität zu verhindern. Folglich müssen entsprechende Informationen veröffentlicht werden, die es beispielsweise für externe BioethikerInnen möglich machen, die Vulnerabilität von ProbandInnen zu überprüfen. 


\subsection{Praktische Implikationen für die Forschungsethik}

Das Konzept der Vulnerabilität muss als ein normatives Konzept verstanden werden. Dies bedeutet, dass Personen, die in einem bestimmten Kontext als vulnerabel identifiziert wurden, das Recht auf adäquate Schutzmaßnahmen haben, um diese Vulnerabilität zu reduzieren. Ein Konzept von Vulnerabilität, das ausschließlich deskriptiv bestimmte Personen oder Personengruppen identifiziert, die eine erhöhte Wahrscheinlichkeit aufweisen Unrecht zu erfahren, jedoch keine Anwendung von Schutzmaßnahmen fordert, ist nicht sinnvoll. Daher sollen in diesem Kapitel einige Überlegungen zur praktischen Umsetzung des Konzepts der Vulnerabilität in der Forschungsethik diskutiert werden. Hierzu soll zunächst mit Blick auf die Ergebnisse beider Fallstudien die Frage beantwortet werden, inwiefern Frauen in Indien im Kontext transnationaler Biomedizin eine vulnerable Gruppe darstellen und wie hiermit in der Praxis transnationaler Forschung in Indien umgegangen werden könnte (Kapitel 6.2.1). Daraufhin sollen mögliche Schutzmaßnahmen anhand der Vulnerabilitätskriterien für Forschung mit Frauen im Kontext transnationaler Biomedizin vorgestellt und mit Blick auf die Autonomie von Probandinnen kritisch erörtert werden (Kapitel 6.2.2).

\subsubsection{Frauen in Indien als vulnerable Gruppe im Kontext transnationaler Biomedizin}

Zunächst lässt sich festhalten, dass ethische Aspekte im Rahmen von Forschung mit Frauen in den in Kapitel 4.1 vorgestellten Ethikrichtlinien, zu wenig beziehungsweise in der Deklaration von Helsinki gar keine Beachtung finden. In den Richtlinien sowohl der CIOMS als auch der Erklärung der UNESCO werden diesbezügliche Aspekte zwar aufgegriffen, allerdings finden gerade soziale Bedingungen, die im Kontext von Forschung mit Frauen eine Rolle spielen könnten, zu wenig Berücksichtigung. Mit Blick auf die Analysen der Fallstudien und Aspekte, die bei der Überprüfung der Vulnerabilitätskriterien teilweise ausschlaggebend dafür sind, ob diese zutreffen oder nicht, sind jedoch gerade soziale Faktoren, wie beispielsweise der Bildungsstand, von enormer Bedeutung.

Zur konkreten Frage, ob jedoch gerade Frauen in Indien eine vulnerable Gruppe darstellen, muss an dieser Stelle wiederholt werden, dass zumindest die Probandinnen beider Fallstudien als vulnerabel identifiziert wurden und ihnen somit besonderer Schutz zustünde. Hierbei gilt es jedoch zu beachten, dass mögliche Schutzmaßnahmen zur Reduzierung der Vulnerabilität der Probandinnen der Fallstudie jeweils an die Ursache der Vulnerabilität angepasst sein sollten (Levine et al. 2004). Führt beispielsweise ein ethisch ungültiger informed consent zu Vulnerabilität, könnte dies möglicherweise durch eine Verbesserung des informed consent-Prozesses verhindert werden.

Anhand der Ergebnisse der Fallstudien können allerdings keine allgemeingültigen Aussagen über 
Frauen in Indien als vulnerable Gruppe per se getroffen werden. Wie bereits in Kapitel 6.1 erläutert, besteht eine sinnvolle Anwendung des Konzepts der Vulnerabilität nicht darin, eine fixe Liste mit vulnerablen Gruppen zu erstellen, da dies die Gefahr der Stereotypisierung und Einschränkung der Autonomie der betroffenen Personen mit sich bringt. Eine mögliche Vulnerabilität von Frauen in Indien, die an medizinischen Studien teilnehmen, wie sie bei den Fallstudien festgestellt wurde, sollte folglich je nach Studienkontext geprüft werden. Das in dieser Arbeit angewendete Konzept der Vulnerabilität bezieht allerdings auch personenbezogene Aspekte in die Beurteilung von Vulnerabilität mit ein. Betrachtet man nun bestimmte Eigenschaften und Lebensbedingungen, die zu Vulnerabilität einer Person oder Personengruppe führen könnten, wie beispielsweise die Anfälligkeit einem unfairen Nutzen im Rahmen einer Studienteilnahme zuzustimmen und somit ausgebeutet zu werden, ist zu vermuten, dass diese auf viele Frauen in Indien zutreffen (siehe hierzu Kapitel 4.2.2). Zu dieser Annahme führen insbesondere Informationen und Daten über die sozioökonomische Situation, den Bildungsstatus und anhaltende Diskriminierung und Ausübung von Gewalt gegenüber vielen Frauen in Indien. Diese Informationen weisen zudem darauf hin, dass die Wahrscheinlichkeit, dass Frauen in Indien in einem bestimmten Studienkontext vulnerabel sein könnten, höher ist als die Wahrscheinlichkeit, dass Männer in Indien im Zuge einer Studienteilnahme vulnerabel sind. Demnach könnte man dafür plädieren, dass Frauen in Indien im Rahmen transnationaler Forschung generell gewisse Schutzmaßnahmen erhalten sollten. Es ist beispielsweise sinnvoll, mehr Transparenz bezüglich der Probandinnengruppe, deren Lebenssituationen und potenziellem Nutzen für die Probandinnen zu fordern, um überprüfen zu können, ob im Kontext der Studienteilnahme Vulnerabilität vorliegt. Falls dies zutrifft könnten gezielte Schutzmaßnahmen etabliert werden. Die Forderung nach mehr Transparenz ist bereits eine gewisse Form von Schutz und soll deswegen in einem späteren Abschnitt genauer erläutert werden.

\subsubsection{Praktische Anwendung des Vulnerabilitätskonzepts: Schutzmaßnahmen versus Respekt für Autonomie}

In diesem Kapitel sollen allgemeine Aspekte der praktischen Anwendung des Vulnerabilitätskonzepts diskutiert werden. Hierzu gehört die Erläuterung möglicher Schutzmaßnahmen und inwiefern diese die Autonomie von ProbandInnen einschränken könnten.

Eine Möglichkeit der Anwendung des Konzepts der Vulnerabilität besteht in einer retrospektiven ethischen Bewertung und Analyse von Studien, zum Beispiel wenn hier kontroverse Einschätzungen vorliegen. Eine solche Vorgehensweise wurde anhand der beiden Fallstudien exemplarisch vorgelegt. Hierbei kann allerdings eine mögliche Vulnerabilität von StudienteilnehmerInnen nicht verhindert werden, sondern wird erst nach Abschluss der Studie festgestellt. Außerdem könnte das Konzept der 
Vulnerabilität in der angewandten Forschungsethik zukünftig genutzt werden, um vor Beginn einer Studie vulnerable Personen oder Gruppen zu identifizieren und darauf mit gezielten Schutzmaßnahmen zu reagieren. Diese Vorgehensweise soll verhindern, dass Vulnerabilität entsteht.

\section{Mögliche Schutzmaßnahmen versus Respekt für Autonomie von Studienteilnehmerinnen}

Im Folgenden sollen mögliche konkrete Schutzmaßnahmen für vulnerable ProbandInnen im Kontext transnationaler Biomedizin vorgestellt und dahin überprüft werden, ob diese das ethische Prinzip Respekt für Autonomie abschwächen könnten. Eine mögliche Einschränkung der Autonomie zu erörtern, ist insofern wichtig, da eine bisher gängige Schutzmaßnahme, nämlich der kategorische Ausschluss vulnerabler Gruppen von medizinischer Forschung, enorm die Autonomie dieser Personen missachtet. Diese Personen können dann nämlich nicht selbstbestimmt entscheiden, ob sie an einer Studie teilnehmen wollen oder nicht. Dies wird als problematisch erachtet, weil das Prinzip Respekt für Autonomie ein sehr wichtiges, wenn nicht gar zentrales, Prinzip in der Medizinethik ist.

Zunächst gilt es jedoch das Prinzip Respekt für Autonomie genauer zu beleuchten. Hierzu soll die Herangehensweise der beiden renomierten Bioethikautoren Tom L. Beauchamp und James F. Childress dienen. Diese nennen als Bedingungen für autonome Handlungen von Personen, dass diese intentional handeln, ihre Entscheidungen verstehen und zudem frei von kontrollierenden Einflüssen agieren (Beauchamp und Childress 2013, S. 104). Die Autonomie von handelnden Personen zu respektieren, bedeutet laut Beauchamp und Childress deren Recht anzuerkennen, dass diese Personen Ansichten vertreten, Entscheidungen treffen und beruhend auf deren persönlichen Werten und Vorstellungen handeln können (Beauchamp und Childress 2013, S. 106). Sie fordern, dass das Prinzip Respekt für Autonomie respektvolles Handeln und nicht nur eine respektvolle Einstellung beinhalten müsse (Beauchamp und Childress 2013, S. 106f). Folglich ginge es um mehr als nur um die Nichteinmischung in persönliche Angelegenheiten. Sie ergänzen hierzu: „It includes, in some contexts, building up or maintaining others' capacities for autonomous choice while helping to allay fears and others conditions that destroy or disrupt autonomous action" (Beauchamp und Childress 2013, S. 107). Diese speziellen Erläuterungen zum Prinzip Respekt für Autonomie gilt es bei der Vorstellung von Schutzmaßnahmen im Blick zu behalten.

Die nachfolgende Tabelle dient als Überblick für potenzielle Schutzmaßnahmen, die jeweils den in dieser Arbeit verwendeten Vulnerabilitätskriterien zugeordnet werden. Zusätzlich werden zu den jeweiligen Schutzmaßnahmen Vorschläge für deren praktische Umsetzung vorgestellt. Im Anschluss an die Tabelle werden die einzelnen Schutzmaßnahmen genauer erläutert. 
Tabelle 3: Vulnerabilitätskriterien, Schutzmaßnahmen und Beispiele für deren praktische Umsetzung

\begin{tabular}{|c|c|c|c|}
\hline & Vulnerabilitätskriterien & Schutzmaßnahmen & Beispiele für praktische Umsetzung \\
\hline \multirow{2}{*}{ 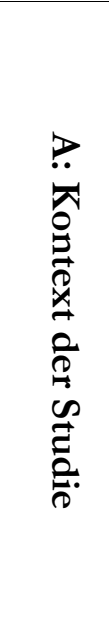 } & $\begin{array}{l}\text { I. Ethisch unzureichende } \\
\text { Konzipierung der Studie }\end{array}$ & $\begin{array}{l}\text { a) Fairen Nutzen gewährleisten } \\
\text { b) Zwang und Manipulation bei Rekrutierung } \\
\text { verhindern } \\
\text { c) Lokale Relevanz der Studie gewährleisten }\end{array}$ & \begin{tabular}{|rl}
$\begin{aligned} Z u \text { a) } \\
\text { - }\end{aligned}$ & Informationen über Art und Ausmaß des Nutzens veröffentlichen \\
- & ProbandInnen bei Beurteilung der Fairness des Nutzens miteinbeziehen \\
Zu b) & \\
- & wenn möglich, nicht durch behandelnde ÄrztInnen rekrutieren \\
Zu c) & \\
- & Forschung betrifft lokale Gesundheitsbedürfnisse \\
- & Plan und Vereinbarungen, wie Ergebnisse der Forschung bereitgestellt \\
& werden (diesbezügliche Informationen veröffentlichen)
\end{tabular} \\
\hline & $\begin{array}{l}\text { II. Ethische Ungültigkeit } \\
\text { des informed consent }\end{array}$ & a) Angemessene Aufklärung & \begin{tabular}{|cl} 
Zu a) & \\
- & Aufklärungsdokumente in Muttersprache der ProbandInnen bereitstellen \\
- & mehr Zeit bei Aufklärung von ProbandInnen mit niedrigem Bildungsstand \\
& einplanen \\
- & Prozess des informed consent und diesbezügliche Dokumente veröffentlichen
\end{tabular} \\
\hline \multirow{3}{*}{ 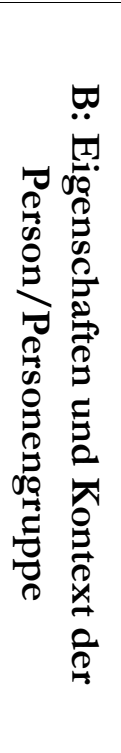 } & $\begin{array}{l}\text { I. Prädisposition, } \\
\text { zusätzliches Leid zu } \\
\text { erfahren }\end{array}$ & $\begin{array}{l}\text { a) Eruieren von vorherigem Leid } \\
\text { b) Vermeiden von zusätzlichem Leid }\end{array}$ & $\begin{array}{l}\text { Zu a und b) } \\
\text { - Informationen über vorheriges physisches oder psychisches Leid und über } \\
\text { sozioökonomischen Hintergrund veröffentlichen } \\
\text { - ProbandInnen bei Bewertung über vorheriges oder potenziell zusätzliches } \\
\text { Leid miteinbeziehen (Bsp.: durch Kurzinterviews) }\end{array}$ \\
\hline & $\begin{array}{l}\text { II. Unfähigkeit, informed } \\
\text { consent zu erteilen } \\
\text { und/oder Anfälligkeit, } \\
\text { unfreiwillig zuzustimmen }\end{array}$ & $\begin{array}{l}\text { a) Verstehen überprüfen } \\
\text { b) Unfreiwilligkeit durch unangemessenen } \\
\text { Anreiz verhindern }\end{array}$ & $\begin{aligned} \text { Zu a) } & \\
\text { - } & \text { Informationen über Bildungsstand der ProbandInnen veröffentlichen } \\
- & \text { Verständnistest } \\
\text { Zu b) } & \\
\text { - } & \text { Aufwandsentschädigung an sozioökonomische Situation der ProbandInnen } \\
& \text { anpassen und diesbezügliche Informationen veröffentlichen }\end{aligned}$ \\
\hline & $\begin{array}{l}\text { III. Anfälligkeit, } \\
\text { ausgebeutet zu werden }\end{array}$ & $\begin{array}{l}\text { a) Einschätzen der Wahrscheinlichkeit, dass } \\
\text { ProbandInnen einer ausbeutenden Situation } \\
\text { zustimmen würden } \\
\text { b) Fairen Nutzen gewährleisten }\end{array}$ & $\begin{array}{cl}\mathrm{Zu} \mathrm{a} \text { ) } & \\
\text { - } & \text { Informationen über sozioökonomischen Hintergrund der ProbandInnen } \\
& \text { veröffentlichen } \\
\mathrm{Zu} \mathrm{b)} & \\
- & \text { s.o. }\end{array}$ \\
\hline
\end{tabular}


Vor der Erläuterung der einzelnen Schutzmaßnahmen soll an dieser Stelle betont werden, dass eine wichtige Form der praktischen Umsetzung für viele der oben genannten Schutzmaßnahmen das Erheben und Offenlegen bestimmter Informationen und Daten bezüglich der jeweiligen ProbandInnen ist. Mithilfe solcher Informationen sollte es möglich sein, eine mögliche Vulnerabilität potenzieller ProbandInnen besser zu überprüfen, um noch vor Beginn einer Studienteilnahme gegebenenfalls gezielte Schutzmaßnahmen bereitzustellen. Außerdem würde die Transparenz bezüglich Informationen, mithilfe derer die ethischen Aspekte einer Studie besser beurteilbar wären, zeigen, dass die ForscherInnen und SponsorInnen den ethischen Standards eine gewisse Bedeutung beimessen. Die Veröffentlichung bestimmter Aspekte, die auf Forschung mit möglicherweise vulnerablen Personen hinweisen, übt insofern Druck auf die ForscherInnen und SponsorInnen aus, als dass diese aufzeigen müssten, wie sichergestellt wurde, dass diesen ProbandInnen durch ihre Studienteilnahme kein Unrecht und/oder Leid widerfahren ist. Somit nimmt das Offenlegen von bestimmten Informationen die Funktion einer gewissen Selbstkontrolle für ForscherInnen und SponsorInnen ein.

\section{A: Kontext der Studie}

\section{Ethisch unzureichende Konzipierung der Studie}

\section{a) Fairen Nutzen gewährleisten:}

Es gilt zu beachten, dass die Schutzmaßnahme fairen Nutzen gewährleisten zwei Vulnerabilitätskriterien (siehe Tabelle 3) zugeordnet wurde, da sowohl das Vulnerabilitätskriterium Ethisch unzureichende Konzipierung der Studie als auch Anfälligkeit, ausgebeutet zu werden Ausbeutung verhindern sollen.

Eine Schutzmaßnahme zur Verhinderung von Ausbeutung ist somit die Gewährleistung eines fairen Nutzens für die ProbandInnen. Hier können wie bereits erläutert verschiedene Arten von Nutzen zum Tragen kommen. Die Fairness des Nutzens gilt es als unterschiedlich zu beurteilen, wenn dieser nur für den Zeitraum der Studienteilnahme oder darüber hinausgehend gewährleistet wird. Außerdem sollte mitberücksichtigt werden, ob der Nutzen nur den ProbandInnen der Studie zur Verfügung gestellt wird oder aber der ganzen Bevölkerung eines Gastlandes.

Zudem sollten ausreichend Informationen zu möglichen Nutzen und Vorteilen für StudienteilnehmerInnen bereitgestellt werden. Hierzu zählen beispielsweise Informationen über die Bereitstellung von Krankenversicherung, kostenloser medizinischer Versorgung und Bereitstellung des möglicherweise als erfolgreich getesteten Medikaments. Hinzu kommen detaillierte Angaben zu den Bedingungen solcher Bereitstellungen. Hierzu zählen die Dauer dieser Bereitstellungen sowie Informationen darüber, für wen beispielsweise ein als erfolgreich getestetes Medikament bereitgestellt wird (nämlich ob dies nur für die ProbandInnen oder eventuell für die gesamte Bevölkerung des Gastlandes gilt und zu welchem Preis).

Die Gewährleistung eines fairen Nutzens als eine mögliche Schutzmaßnahme könnte die Autonomie von ProbandInnen einschränken, wenn diese bei der Beurteilung der Fairness des Nutzens nicht 
miteinbezogen würden. Folglich würden nämlich ForscherInnen und SponsorInnen entscheiden, welcher Nutzen angemessen ist und hierbei womöglich die Perspektive der ProbandInnen missachten. Bei der Anwendung eines fairen Nutzens als Schutzmaßnahme für vulnerable ProbandInnen ist somit deren Mitspracherecht zwingend notwendig. Diesbezüglich gibt es den Lösungsansatz sogenannter Community advisory boards, der in Kapitel 6.3 noch genauer erläutert wird.

\section{b) Zwang und Manipulation bei Rekrutierung verbindern:}

Eine weitere Schutzmaßnahme, die Vulnerabilität hinsichtlich der Studienkonzipierung verhindern soll, betrifft die Rekrutierung von ProbandInnen. Im Vordergrund steht hierbei, ob diese zum Beispiel von ihren behandelnden ÄrztInnen oder von Personen, die für Clinical Research Organizations (CRO) arbeiten, gezielt angesprochen und rekrutiert wurden oder ob die Probandinnen durch ein Plakat, Flyer oder ähnliches auf die Studie aufmerksam wurden. Den Prozess der Rekrutierung betreffend könnte nämlich die Sorge einer Zustimmung unter Zwang oder Manipulation entstehen, wenn möglicherweise Vertrauenspersonen wie ÄrztInnen zu einer Teilnahme raten. Demnach wäre es sehr hilfreich, wenn routinemäßig offengelegt würde, wie die ProbandInnen rekrutiert wurden und gegebenenfalls mit welchen Maßnahmen Zwang und/oder Manipulation bei der Rekrutierung unterbunden wurden.

\section{c) Lokale Relevanz der Studie gewährleisten:}

Um eine unethische Konzipierung einer Studie im Rahmen transnationaler Biomedizin zu verhindern, muss ein Forschungsvorhaben die lokalen Gesundheitsbedürfnisse des Gastlandes adressieren. Zusätzlich muss sichergestellt werden, dass die Ergebnisse beziehungsweise sich als wirksam herausgestellte Interventionen den ProbandInnen oder gar der ganzen Bevölkerung bereitgestellt werden. Diesbezügliche Pläne und Vereinbarungen, beispielsweise zu welchem Preis ein neu entwickeltes Medikament zur Verfügung gestellt wird, sollten außerdem veröffentlicht werden.

\section{Ethische Ungültigkeit des informed consent}

a) Angemessene Aufklärung:

Zur Verhinderung einer möglichen Vulnerabilität von ProbandInnen sollte die Aufklärung gezielt an deren Bedürfnisse angepasst werden. Besteht beispielsweise die Sorge, dass Aufklärungsdokumente aus sprachlichen Gründen nicht ausreichend verstanden würden, müssen diese in der Muttersprache der ProbandInnen bereitgestellt werden. Zudem sollte für die Aufklärung von ProbandInnen mit einem niedrigen Bildungsstand oder AnalphabetInnen im Vergleich zu ProbandInnen mit einem hohen Bildungsstand mehr Zeit eingeplant werden, da hierdurch die Wahrscheinlichkeit erhöht wird, dass alle wichtigen Informationen verstanden werden können. Zusätzlich sollten spezielle Aufklärungsdokumente für solche ProbandInnen entwickelt werden, die beispielsweise relevante Information mithilfe von Bildern darstellen. Des Weiteren sollte der informed consent-Prozess transparenter dargestellt 
werden. Hierzu könnte man eventuell standardmäßig die Aufklärungs- und Einwilligungsunterlagen als Zusatzmaterial veröffentlichen.

\section{B: Eigenschaften und Kontext der Person/Personengruppe}

\section{Prädisposition, zusätzliches Leid zu erfahren}

a) Eruieren von vorberigem Leid:

Es gilt, vorheriges Leid von ProbandInnen zu ermitteln, um die Wahrscheinlichkeit für zusätzliches Leid einschätzen zu können und folglich auch verhindern zu können. Vorheriges Leid kann beispielsweise in Form von physischem oder psychischem Leid vorhanden sein. Demnach sollten sowohl Aspekte, die den Gesundheitszustand der ProbandInnen betreffen, als auch Aspekte, die Leid im Sinne einer schwierigen sozioökonomischen Situation meinen, berücksichtigt und gezielt erfragt werden.

\section{b) Vermeiden von zusätzlichem Leid:}

Der zweite Schritt zum Schutz von ProbandInnen, die eine erhöhte Wahrscheinlichkeit Leid zu erfahren aufweisen, ist das gezielte Verhindern von zusätzlichem Leid unter Berücksichtigung von zuvor festgestelltem vorherigem Leid. Vorheriges Leid, könnte beispielsweise ein schlechter Gesundheitszustand von ProbandInnen aufgrund von Mangelernährung sein. Diese ProbandInnen könnten dann durch potenzielle Nebenwirkungen möglicherweise stärker körperlich geschwächt werden im Vergleich zu ProbandInnen, die eine Studienteilnahme in völlig gesundem Zustand beginnen. Des Weiteren kann vorheriges Leid aufgrund einer prekären sozioökonomischen Situation bestehen. Bei Studien, die Krankheiten erforschen, die gesellschaftlich besonders stigmatisierend sein können, wie beispielsweise HIV/AIDS, könnten solche ProbandInnen zusätzliches Leid erfahren, wenn deren Anonymität im Rahmen der Studien nicht ausreichend gewährleistet wird. So könnten sie unter bestimmten Umständen beispielsweise ihren Arbeitsplatz verlieren und hierdurch zusätzliches Leid erfahren, wenn bekannt wird, dass sie an einer HIV-Studie teilnehmen. Um dies zu verhindern, könnte man eventuell Maßnahmen zur Wahrung der Anonymität verstärken.

Die Eruierung von vorherigem Leid und von Risiken für zusätzliches Leid sollte unter Miteinbeziehung potenzieller ProbandInnen erfolgen. Man könnte beispielsweise im Rahmen der Rekrutierung Kurzinterviews mit potenziellen ProbandInnen führen, um bereits bestehendes Leid und mögliche Sorgen hinsichtlich einer Studienteilnahme abzufragen.

Anzeichen für die eine Abschwächung der Autonomie von ProbandInnen durch die Schutzmaßnahmen eruieren von vorherigen Leid und vermeiden von zusätzlichem Leid, gibt es keine. 


\section{Unfähigkeit, informed consent zu erteilen und/oder Anfälligkeit, unfreiwillig zuzustimmen}

\section{a) Verstehen überprüfen:}

Das Verstehen studienrelevanter Informationen der potenziellen Probandinnen vor der eigentlichen Einwilligung gezielt zu überprüfen, ist eine weitere Schutzmaßnahme im Rahmen des informed consentProzesses. Dies könnte beispielsweise anhand eines Verständnistestes, wie dies in Fallstudie A durchgeführt wurde, oder durch präzises Nachfragen der aufklärenden Person geschehen. Um das Verstehen von ProbandInnen im Voraus besser einschätzen zu können, sollten Daten zum Bildungsstatus der jeweiligen ProbandInnen erhoben und auch veröffentlicht werden. Darüber hinaus sollte offengelegt werden, wie sichergestellt wurde, dass studienrelevante Informationen ausreichend verstanden wurden, wenn beispielsweise Personen mit einem sehr niedrigen Bildungsstatus an einer Studie teilnehmen.

\section{b) Unfreiwilligkeit durch unangemessenen Anreiz verbindern:}

Bei der Festlegung eines fairen Nutzens sollte darauf geachtet werden, dass im Falle eines sehr hohen Nutzens, wie zum Beispiel der Zusicherung lebenslang krankenversichert zu werden oder dem Anbieten einer verhältnismäßig hohen Aufwandsentschädigung, dieser zu einem unangemessenen Anreiz werden könnte. Dies gilt es zu verhindern, da hierdurch die Freiwilligkeit der ProbandInnen eingeschränkt werden könnte. Zudem sollte offengelegt werden, ob eine Aufwandsentschädigung gezahlt wurde. Um die Sorge eines unangemessenen Anreizes und damit möglicherweise Unfreiwilligkeit beurteilen zu können, muss hierbei auch die Höhe der Aufwandsentschädigung genannt und das Verhältnis zum Einkommen der ProbandInnen dargestellt werden.

Es gibt keine Hinweise darauf, dass den informed consent-Prozess betreffende Schutzmaßnahmen (angemessene Aufklärung, Verstehen überprüfen und Unfreiwilligkeit durch unangemessenen Anreiz verbindern) die Autonomie von ProbandInnen einschränken könnten. Vielmehr sind diese Maßnahmen eher fördernd für die Autonomie von ProbandInnen, da eine angemessene Aufklärung, das Sicherstellen, dass alle Informationen verstanden wurden und das Vermeiden eines unangemessenen Anreizes, eine wichtige Grundlage für eine selbstbestimmte Entscheidung darstellen.

\section{Anfälligkeit, ausgebeutet zu werden}

a) Einschätzen der Wabrscheinlichkeit, dass ProbandInnen einer ausbeutenden Situation zustimmen würden:

Um einschätzen zu können, inwiefern ProbandInnen anfällig für Ausbeutung sein könnten, müssen Informationen und Daten zu deren sozioökonomischem Status erhoben und auch veröffentlicht werden. Informationen, die diesbezüglich wichtig wären, sind beispielsweise der Bildungsstand, das Einkommen und der Beruf von ProbandInnen. Eine prekäre sozioökonomische Situation könnte nämlich die Wahrscheinlichkeit erhöhen, dass ProbandInnen in eine ausbeutende Transaktion 
einwilligen. Zeigt sich anhand der oben genannten Informationen eine erhöhte Wahrscheinlichkeit für Ausbeutung, sollte dem verstärkt durch das Gewährleisten eines Fairen Nutæens (Punkt b) entgegengewirkt werden.

\section{b) Fairen Nutzen gewährleisten:}

Diese Schutzmaßnahme wurde bereits bezüglich Ethisch un₹ureichende Kon₹ipierung der Studie erläutert.

\section{Ausschluss von Forschung}

Als letzte mögliche Schutzmaßnahme soll an dieser Stelle der Ausschluss vulnerabler Personen von medizinischer Forschung genannt werden. Wenn von einer sehr starken Form von Vulnerabilität auszugehen ist, da beispielsweise alle Vulnerabilitätskriterien zutreffend sind oder die ProbandInnen einer sehr hohen Wahrscheinlichkeit ausgesetzt sind, erhebliches Unrecht und/oder Leid zu erfahren, kann es sinnvoll sein diese ProbandInnen von einer Studienteilnahme auszuschließen. Hierbei wird allerdings die Autonomie der ProbandInnen stark eingeschränkt, da man ihnen das Recht absprechen würde, selbstbestimmt für oder wider eine Studienteilnahme entscheiden zu können. Deswegen muss hierzu klargestellt werden, dass dies erst als Schutzmaßnahme angewendet werden sollte, wenn zuvor alle anderen möglichen Schutzmaßnahmen gescheitert sind. Der Ausschluss vulnerabler Gruppen von medizinischer Forschung als Schutzmaßnahme wird in der Tabelle nicht aufgeführt, da sie nicht einzelnen Vulnerabilitätskriterien zugeordnet werden kann.

Abschließend soll festgehalten werden, dass die vorgestellten Schutzmaßnahmen keinen Anspruch auf Vollständigkeit verfolgen. Es ist möglich, dass je nach Ursache für Vulnerabilität weitere Schutzmaßnahmen sinnvoll sein könnten. Insgesamt soll in dieser Arbeit ein Konzept von Vulnerabilität gestärkt werden, das nicht nur als Rechtfertigung dient, um bestimmte Personengruppen von medizinischer Forschung auszuschließen. Zugegebenermaßen stellt beispielsweise die Forderung nach mehr Transparenz vermutlich einen weit größeren Aufwand dar als die Maßnahme bestimmte Personen von Studien per se auszuschließen. Allerdings stehen die meisten der genannten Schutzmaßnahmen nicht im Gegensatz zum Prinzip Respekt für Autonomie und sollten deswegen zunächst dem Ausschluss bestimmter Personengruppen von Forschung vorgezogen werden, da hierdurch die Autonomie dieser Personen nur bedingt oder gar nicht eingeschränkt würde. 


\subsection{Ethische Konflikte im Kontext transnationaler Biomedizin: Ein Ausblick}

Anhand der vorgelegten Fallstudien-Analysen konnte gezeigt werden, dass die Sorge, Frauen in Indien könnten im Kontext transnationaler Biomedizin potenziell vulnerabel sein, nicht unbegründet ist. Darüber hinaus konnte dargestellt werden, dass das Konzept der Vulnerabilität sinnvoll verwendet werden kann, um ethische Aspekte im Rahmen internationaler Forschung zu diskutieren. Allerdings ist dies nur möglich, wenn ausreichende Informationen über die StudienteilnehmerInnen und die sonstigen Bedingungen der Studie, wie beispielsweise der Nutzen für die ProbandInnen, offengelegt werden. Insgesamt wurde in Kapitel 3.3 deutlich, dass transnationale Biomedizin mit hohem ethischem Konfliktpotenzial einhergeht. Einige Sorgen diesbezüglich, wie beispielsweise die potenzielle Ausbeutung von Menschen in Entwicklungsländern, können mithilfe des Konzepts der Vulnerabilität verbessert werden.

Da wir mit der Forschungsfrage nach Vulnerabilität im Kontext transnationaler Biomedizin noch am Anfang stehen, ist es dringend notwendig weitere Fallstudien zu analysieren. An dieser Stelle sollen jedoch für die zukünftige Arbeit von BioethikerInnen, ForscherInnen, SponsorInnen und HerausgeberInnen zwei Forderungen genannt werden, die sich bei einer ethischen Bewertung internationaler Forschung in dieser Arbeit bereits als besonders wichtig herausstellten. Die erste ist die Forderung nach mehr Transparenz hinsichtlich Informationen und Aspekten, die eine Überprüfung einer potenziellen Vulnerabilität von ProbandInnen überhaupt erst möglich machen. Durch eine verstärkte Öffentlichkeit hinsichtlich internationale Forschung mit potenziell vulnerablen Personen könnte der Druck auf SponsorInnen und ForscherInnen erhöht werden, auf eine mögliche Vulnerabilität der ProbandInnen angemessen reagieren zu müssen. Diese Forderung könnte zudem von den HerausgeberInnen wissenschaftlicher Journals gestellt werden. Hierdurch könnten die HerausgeberInnen beeinflussen, dass nur solche Studien im Kontext transnationaler Biomedizin veröffentlicht werden, bei denen Prioritäten bei den ethischen Aspekten einer Studiendurchführung und bei dem Schutz der ProbandInnen gesetzt wurden. Ein Beispiel für Leitlinien zur Veröffentlichung von Studien ist das Consort Statement der Consort Group (Consolidated Standards of Reporting Trials). Dies ist eine Empfehlung anhand einer 25-Punkte-Checkliste zur Veröffentlichung von randomisierten Studien (CONSORT). Das Consort Statement enthält allerdings keine Forderungen zur Veröffentlichung beispielsweise sozioökonomischer Daten von ProbandInnen oder ethischer Aspekte im Rahmen der Studiendurchführung (CONSORT 2010). Es wäre ethisch geboten, dass das Consort Statement seine Kriterien überdenkt und solchen ethischen Überlegungen noch mehr Gewicht einräumt.

Die zweite Forderung besteht darin, dem Mitspracherecht von StudienteilnehmerInnen bei der Beurteilung ethischer Aspekte im Rahmen internationaler Forschung, wie beispielsweise der Einschätzung eines fairen Nutzens, einen höheren Stellenwert beizumessen. Hierdurch könnte verhindert werden, dass nur Personen aus Industriestaaten bewerten, ob Menschen in Entwicklungsländern 
besonderen Schutz benötigen und welche Form von Schutz angemessen sei. Die bisher einseitige Praxis ist insofern problematisch, da Personen mit unterschiedlichem gesellschaftlichem und kulturellem Hintergrund die Angemessenheit ethischer Standards und der Anwendung bestimmter Schutzmaßnahmen vermutlich unterschiedlich bewerten würden. Ein möglicher Versuch die Mitbestimmung von betroffenen Personen beziehungsweise communities im Rahmen transnationaler Biomedizin zu gewährleisten ist das Etablieren sogenannter community advisory boards (CAB). Diese sollen vor allem auch dazu dienen, Ausbeutung zu reduzieren oder gar zu verhindern (Pratt et al. 2013). CABs bestehen aus Mitgliedern der community, aus der die Forschungspopulation stammt. CABs können in erster Linie eine beratende Funktion für das Forscherteam ausführen oder aber ihr Einfluss reicht so weit, dass sie partnerschaftlich in Entscheidungen, die das Forschungsprojekt betreffen, miteinbezogen sind (Pratt et al. 2013). Pratt et al. fanden in einer Studie über ein CAB in Thailand folgendes heraus:

„After two years in operation members have gained knowledge and developed capacities that are foundational for one-day taking on a role to reduce exploitation, their ability to critically evaluate studies for the presence of exploitative elements has not yet been strongly demonstrated" (Pratt et al. 2013, S. 1).

Folglich muss das Etablieren von CABs als ein Lösungsansatz für die Mitbestimmung von communities auch kritisch betrachtet werden und erfordert noch weitere Untersuchungen.

Um unethische Forschung in Entwicklungsländern zu verhindern und potenzielle ProbandInnen vor Ausbeutung zu schützen, könnte man aus theoretischer Sicht dafür plädieren, dass die Durchführung transnationaler Biomedizin von den entsprechenden Regierungen unterbunden wird. Dies würde allerdings die Autonomie ganzer Bevölkerungen einschränken und eine weitreichende Stereotypisierung von StudienteilnehmerInnen in Entwicklungsländern bedeuten. Unabhängig von möglichen wirtschaftlichen Vorteilen kann transnationale Forschung für diese Länder nämlich weitere positive Aspekte mit sich bringen, wie beispielsweise den Aufbau einer medizinischen Infrastruktur oder die Forschung zu lokal relevanten Erkrankungen und die anschließende Bereitstellung von Medikamenten, die sich als sicher und wirksam herausstellten. Folglich sollte transnationale Biomedizin nicht verboten werden, sondern vielmehr kritisch darauf geachtet werden, dass hohe ethische Standards gesetzt werden, um zu verhindern, dass ProbandInnen Unrecht widerfährt. Findet transnationale Forschung nun mit Frauen in Entwicklungs- oder Schwellenländern mit patriarchalen Gesellschaftsstrukturen statt, kann es sinnvoll sein, die ethischen Standards noch strenger zu überprüfen. Anhand Fallstudie A zeigten sich zwar keine großen Unterschiede zwischen der Vulnerabilität der männlichen und weiblichen StudienteilnehmerInnen, allerdings wiesen beispielsweise die Studienteilnehmerinnen einen durchschnittlich niedrigeren Bildungsstand auf im Vergleich zu den Studienteilnehmern. Insgesamt sollte aufgrund gesellschaftlicher Bedingungen, die die Wahrscheinlichkeit erhöhen, dass gerade Frauen im Rahmen transnationaler Biomedizin Unrecht und/oder Leid erfahren könnten, eine besonders 
ausführliche Bewertung ethischer Aspekte bei Forschung mit Frauen in Entwicklungsländern durchgeführt werden.

Bei der Vorgehensweise dieser Arbeit gilt es als kritisch zu beurteilen, dass die Probandinnen der Fallstudien anhand der Analyse und Interpretation als vulnerabel im Kontext ihrer Studienteilnahme bewertet wurden, die Probandinnen selbst hierzu jedoch nicht befragt wurden. Es ließ sich daher dringender Forschungsbedarf aus ethisch-empirischer Sicht formulieren, Interviewstudien mit Probandinnen durchzuführen, um ihre Perspektive noch abzufragen. Diese Arbeit könnte jedoch eine wichtige Grundlage für eine weitere Auseinandersetzung und Diskussion zum Thema Vulnerabilität von Frauen in Indien im Kontext transnationaler Biomedizin bieten und folglich als theoretischer Hintergrund verwendet werden, um zukünftig solche empirischen Studien mit Frauen durchzuführen. Denn hiermit könnte die Forderung nach mehr Mitspracherecht möglicherweise erfüllt werden und geprüft werden, inwiefern die Probandinnen selbst davon ausgehen, dass ihnen mit einer erhöhten Wahrscheinlichkeit Unrecht und/oder Leid widerfahren könnte und auch, welche Schutzmaßnahmen sie als sinnvoll erachten würden. Diese Arbeit soll dazu dienen, für ethische Aspekte im Kontext transnationaler Biomedizin zu sensibilisieren. Hierbei stehen vor allem die Forderungen nach mehr Transparenz und nach der Einrichtung von Mitteln, die das Mitspracherecht der jeweils betroffenen ProbandInnen gewährleisten, im Vordergrund. Abschließend lässt sich festhalten, dass das Konzept der Vulnerabilität bei der ethischen Beurteilung transnationaler Biomedizin hilfreich sein kann, um StudienteilnehmerInnen vor Unrecht und Leid zu schützen. 


\section{Zusammenfassung}

Transnationale Biomedizin ist in den letzten Jahren enorm gewachsen, wobei Indien einen besonders beliebten Zielort für die Auslagerung klinischer Studien darstellt. Zahlreiche forschungsethische Konflikte gehen mit transnationaler Biomedizin einher. Da internationale Ethikrichtlinien, wie beispielsweise die Deklaration von Helsinki, nur sog. soft law darstellen, verstärkt sich die Problematik, gewisse ethische Standards für transnationale Biomedizin zu gewährleisten. Folglich sollen weitere Kriterien für die ethische Bewertung solcher Studien untersucht werden. Das Konzept der Vulnerabilität bietet hierzu einen wichtigen neuen Ansatz, der es erlaubt, auch sozioökonomische Aspekte von ProbandInnen miteinzubeziehen. In patriarchal-strukturierten Gesellschaften, auch Indien zählt tendenziell zu einer solchen Gesellschaft, könnten gerade Frauen aufgrund sozialer Faktoren besonders vulnerabel sein. Demnach soll in dieser Arbeit das Konzept der Vulnerabilität im Kontext transnationaler Biomedizin am Beispiel von Frauen in Indien analysiert werden.

Da Vulnerabilität bisher weder einheitlich definiert noch operationalisiert ist, werden zunächst spezifische Vulnerabilitätskriterien für den Kontext dieser Arbeit erarbeitet. Anhand der exemplarischen Anwendung dieser Vulnerabilitätskriterien soll geprüft werden, inwiefern das Konzept der Vulnerabilität geeignet sein könnte, ethische Aspekte im Kontext transnationaler Biomedizin mit Frauen zu bewerten. Hierzu wurden mithilfe von Registrierungsplattformen für klinische Studien zwei transnationale Studien ausgewählt, die mit Frauen in Indien durchgeführt wurden und als Fallstudien für diese Arbeit dienen.

Das verwendete Hauptvulnerabilitätskriterium ist die erböhte Wabrscheinlichkeit, Unrecht ұn erfahren und erfordert zur Konkretisierung weitere Vulnerabilitätskriterien. Diese sind:
A: Kontext der Studie

A: Kontext der Studie
I. Ethisch unzureichende Konzipierung der Studie
II. Ethische Ungültigkeit des informed consent

B: Eigenschaften und Kontext der Person/ Personengruppe

I. Prädisposition, zusätzliches Leid zu erfahren II. Unfähigkeit, informed consent zu erteilen und/oder Anfälligkeit, unfreiwillig zuzustimmen III. Anfälligkeit, ausgebeutet zu werden

Die erste untersuchte Fallstudie, eine HIV-Impfstudie, wurde mit hohen ethischen Standards durchgeführt. Bei der zweiten Fallstudie, eine HPV-Impfstudie, standen ethische Aspekte weniger im Vordergrund. Zusammenfassend lässt sich feststellen, dass bei beiden Fallstudien die ProbandInnen anhand der angewendeten Vulnerabilitätskriterien als vulnerabel gelten.

Abschließend zeigt sich, dass Vulnerabilitätskriterien sowohl den Kontext einer Studie als auch die Eigenschaften und die Lebenssituation von Personen miteinbeziehen sollten. Die erarbeiteten Vulnerabilitätskriterien stellten sich als gut anwendbar für den Kontext transnationaler Biomedizin dar, 
sind jedoch nicht universal für alle vulnerablen Gruppen einsetzbar. Hieraus ergibt sich die Forderung, jeweils spezifische Vulnerabilitätskriterien für verschiedene Gruppen zu erarbeiten. Zudem zeigt sich, dass das Konzept der Vulnerabilität graduelle Unterschiede aufweist. Hieraus ergeben sich auch unterschiedliche Schutzmaßnahmen. Der bisher übliche Umgang mit vulnerablen Gruppen, nämlich den Ausschluss von Forschung, ist nur zulässig, wenn alle anderen Schutzmaßnahmen versagen, da sonst die Autonomie von ProbandInnen eingeschränkt würde. 
8. Anhang

$\underline{8.1 \text { Abbildungsverzeichnis }}$

Abb. 1: Vulnerabilitätskriterien

S. 30

$\underline{8.2 \text { Tabellenverzeichnis }}$

Tab. 1: Vulnerabilität der StudienteilnehmerInnen in Fallstudie A

Tab. 2: Vulnerabilität der Studienteilnehmerinnen in Fallstudie B $\quad$ S. 113

Tab. 3: Vulnerabilitätskriterien, Schutzmaßnahmen und Beispiele für deren praktische S.129 Umsetzung 


\section{Literaturverzeichnis}

All India Democratic Women's Association (2007). http://aidwaonline.org/ [zuletzt geprüft am 20.09.2014]

Andorno R: Warum braucht eine globale Bioethik die Menschenrechte? In: Gibt es eine universale Bioethik? hrsg. v. Biller-Andorno N, Schaber P, Schulz-Baldes A, Mentis, Paderborn 2008, 59-73 Arbeitsgemeinschaft der Wissenschaftlichen Medizinischen Fachgesellschaften: Wir über uns. http://www.awmf.org/ [zuletzt geprüft am 20.09.2014]

Arzneitelegramm (2007): Zweiter HPV-Impfstoff Cervarix. Arzneitelegramm $\underline{38}$, 101-103

Arzneitelegramm (2009): Neue Daten zu HPV-Impfstoffen (Cervarix, Gardasil). Arzneitelegramm $\underline{40}, 71-73$

Auswärtiges Amt (2013): Indien. http://www.auswaertiges-

amt.de/DE/Aussenpolitik/Laender/Laenderinfos/01-Nodes_Uebersichtsseiten/Indien_node.html [zuletzt geprüft am 20.09.2014]

Ballantyne A (2010): How to Do Research Fairly in an Unjust World. Am J Bioeth 10 (6), 26-35

Banerji R. http://genderbytes.wordpress.com/about/ [zuletzt geprüft am 20.09.2014]

Barouch DH (2008): Challenges in the development of an HIV-1 vaccine. Nature $\underline{455}$, 613-619

Beauchamp TL, Childress JF: Principles of biomedical ethics, 7. Auflage; Oxford University Press, New York 2013

Bhatla N, Suri V, Basu P, Shastri S, Datta SK, Bi D, Descamps DJ, Bock HL (2010):

Immunogenicity and safety of human papillomavirus-16/18 AS04-adjuvanted cervical cancer vaccine in healthy Indian women. J Obstet Gynaecol Res $\underline{36}$ (1), 123-132

Birnbacher D (1999): Ethics and Social Science: Which Kind of Co-operation? Ethical Theory Moral Pract 2 (4), 319-336

Borooah VK (2004): Gender bias among children in India in their diet and immunisation against disease. Soc Sci Med $\underline{58}(9), 1719-1731$

Bosch FX, Lorincz A, Muñoz N, Meijer CJLM, Shah KV (2002): The causal relation between human papillomavirus and cervical cancer. J Clin Pathol 55 (4), 244-265

Braune F, Wiesemann C, Biller-Adorno N: Informed Consent und seine Konkretisierung in der internationalen Bioethik: Zur medizinischen Bedeutung von Aufklärung und Zustimmung in Taiwan und Deutschland; In: Gibt es eine universale Bioethik?; hrsg. v. Biller-Andorno N, Schaber P, Schulz-Baldes A, Mentis, Paderborn 2008, 13-156 
Brockhaus Enzyklopädie, 21. Auflage, hrsg. v. Zwahr A, Brockhaus, Leipzig 2005-2006

BUKO: http://www.bukopharma.de/ [zuletzt geprüft am 20.09.2014]

BUKO (2010a): Aufklärung Glückssache: HPV Impf-Studie in Indien. Pharma-Brief, 2-3

BUKO (2010b): Testfeld Indien: Boomender Forschungsmarkt - schwache Kontrolle. Pharma-

Brief, 5-7

Bunch C (1990): Women's rigths as human rights: Toward a Re-Vision of human rights. Hum

Rights Q 12 (486), 61-71

Bundesärztekammer (2013): Der Weltärztebund (World Medical Association/WMA).

http://www.bundesaerztekammer.de/page.asp?his=1.109.8911.8912\&all=true [zuletzt geprüft am 20.09.2014]

Bundesministerium für wirtschaftliche Zusammenarbeit und Entwicklung (2013):

Entwicklungsland. http://www.bmz.de/de/service/glossar/E/entwicklungsland.html [zuletzt geprüft am 20.09.2014]

Central Drug Standard Control Organization: Initiatives, Achievements and Targets: Central Drug Standard Control Organization (2001-2020).

http://cdsco.nic.in/CDSCO $\% 20$ Initiatives $\% 20 \& \% 20$ Targets $\% 20$ for $\% 20$ website $\% 2013-7$ -

2012_17-07-2012.pdf [zuletzt geprüft am 20.09.2014]

Central Drug Standard Control Organization (2004): Good Clinical Practices For Clinical Research In India. http://cdsco.nic.in/html/gcp1.html [zuletzt geprüft am 20.09.2014]

Central Intelligence Agency (2013): The World Factbook: India: Economy overview. https://www.cia.gov/library/publications/the-world-factbook/geos/in.html [zuletzt geprüft am 20.09.2014]

Chorghade GP, Barker M, Kanade S, Fall C H D (2006): Why are rural Indian women so thin? Findings from a village in Maharashtra. Public Health Nutr $\underline{9}$ (1), 9-18

CIOMS: International ethical guidelines for biomedical research involving human subjects; CIOMS, Geneva 2002

Clinical Trials Registry India: About CTRI. http://ctri.nic.in/Clinicaltrials/cont1.php [zuletzt geprüft am 20.09.2014]

Clinical Trials Registry India: Frequently Asked Questions. http://ctri.nic.in/Clinicaltrials/faq.php\#5a [zuletzt geprüft am 20.09.2014] 
ClinicalTrials.gov (2007): Safety and Immunogenicity Study of tgAAC09, a Gag-PR-RT AAV HIV Vaccine.

http://www.clinicaltrials.gov/ct2/show/record/NCT00482027?term=NCT00482027\&rank=1

[zuletzt geprüft am 20.09.2014]

ClinicalTrials.gov (2011): Evaluation of the Immune and Safety Response of GlaxoSmithKline (GSK) Biologicals' HPV Vaccine in Healthy Indian Women.

http://clinicaltrials.gov/ct2/show/NCT00344032?term=Evaluation +of + the + Immune+and + Safet $\mathrm{y}+$ Response+of +GlaxoSmithKline+\%28GSK $\% 29+$ Biologicals $\% 27+$ HPV+Vaccine+in + Healthy + Indian+Women\&rank=1 [zuletzt geprüft am 20.09.2014]

CONSORT. http://www.consort-statement.org/ [zuletzt geprüft am 20.09.2014]

CONSORT (2010): The CONSORT Statement. http://www.consort-statement.org/consortstatement/overview0/ [zuletzt geprüft am 20.09.2014]

Cook RJ: Women's health and human rights: The promotion and protection of women's health through international human rights law; World Health Organization, Geneva 1994

Czerkinsky CC, Nilsson LA, Nygren H, Ouchterlony O, Tarkowski A (1983): A solid-phase enzyme-linked immunospot (ELISPOT) assay for enumeration of specific antibody-secreting cells. J Immunol Methods $\underline{65}$ (1-2), 109-121

Davidson AJ, O'Brien M (2009): Ethics and medical research in children. Paediatr Anaesth 19 (10), 994-1004

DeBruin D (2004): Looking Beyond the Limitations of "Vulnerability": Reforming Safeguards in Research. Am J Bioeth 4 (3), 76-78

Degele N: Gender/Queer Studies: Eine Einführung (Utb.de Bachelor-Bibliothek 2986); Wilhelm Fink, Paderborn 2008

Demberg T, Robert-Guroff M (2012): Controlling the HIV/AIDS epidemic: current status and global challenges. Front Immunol $\underline{3}$ (250), 1-17

Dennerstein L: Women's health - the context: Recent recommendations of United Nations conferences and initiatives of the Commonwealth of Australia; In: Women's rights and bioethics; hrsg. v. Dennerstein L, Baltes MM: UNESCO. Paris 2000, 9-29

Denny CC, Grady C (2006): Clinical research with economically disadvantaged populations. J Med Ethics $\underline{33}(7), 382-385$

Dören M, Gerhardus A, Gerlach FM, Hornberg C, Kochen MM, Kolip P, Ludwig W, Mühlhauser I, Razum O, Rosenbrock R et al.: Wissenschaftler/innen fordern Neubewertung der HPV-Impfung und ein Ende der irreführenden Informationen 2008 
Drugs Controller General India (2013): Panel of experts for constituting independent Expert Committee to examine the SAE (death) occurring during the clinical trials. http://www.cdsco.nic.in/panelof_exprt.pdf [zuletzt geprüft am 20.09.2014]

Eckenwiler L, Ells C, Feinholz D, Schonfeld T (2008): Hopes for Helsinki: reconsidering "vulnerability". J Med Ethics $\underline{34}$ (10), 765-766

Emanuel E, Wendler D, Grady C (2000): What makes clinical research ethical? JAMA $\underline{283}$ (20), $2701-2711$

Emanuel E, Wendler D, Killen J, Grady C (2004): What makes Clinical Research in Developing Countries Ethical?: The Benchmarks of Ethical Research. J Infect Dis 189 (5), 930-937

Emanuel E, Currie X, Herman A (2005): Undue inducement in clinical research in developing countries: is it a worry. Lancet $\underline{366}$ (9482), 336-340

Entwicklungsausschuss der OECD (DAC) (2013): DAC-Liste der Entwicklungsländer und -gebiete (gültig für die Berichtsjahre 2011-2013).

http://www.bmz.de/de/ministerium/zahlen_fakten/DAC_Laenderliste_Berichtsjahre_2011_2013. pdf [zuletzt geprüft am 20.09.2014]

Excler J (2005): AIDS vaccine development: perspectives, challenges \& hopes. Indian J Med Res 121 (4), $568-581$

Excler J, Kochhar S, Kapoor SDS, Bahri J, Ghosh MD, Ganguly N K, Nayyar A, Chataway M (2008): Preparedness for AIDS vaccine trials in India. Indian J Med Res 127 (6), 531-538

Exley C (2011): Aluminium-based adjuvants should not be used as placebos in clinical trials. Vaccine $\underline{29}$ (50), 9289

Ferlay J, Shin H, Bray F, Forman D, Mathers C, Parkin DM (2010): Estimates of worldwide burden of cancer in 2008: GLOBOCAN 2008. Int J Cancer 127 (12), 2893-2917

Flory J, Emanuel EJ (2004): Interventions to Improve Research Participants' Understanding in Informed Consent for Research: A Systematic Review. JAMA 292 (13), 1593-1601

Gbadegesin S, Wendler D (2006): Protecting Communities in Health Research From Exploitation. Bioethics $\underline{20}$ (5), 248-253

George, T (2011): Institutional ethics committees: critical gaps. Indian J Med Ethics $\underline{8}$ (4), 200-201

George T, Srinivasan S (2006): Does the TGN1412 trial disaster have lessons for clinical trials in India? Natl Med J India 19 (5), 283-286

Gerhardus A (2009): Wie wirksam ist die HPV-Impfung? Deutsches Ärzteblatt 106 (8), 330-334 
Gerhardus A, Razum O (2010): A long story made too short: surrogate variables and the communication of HPV vaccine trials results. J Epidemiol Community Health $\underline{64}$ (5), 377-378 German Commission for UNESCO: Bioethics. http://www.unesco.de/bioethik.html?\&L=1 [zuletzt geprüft am 20.09.2014]

GlaxoSmithKline (2004): Fachinformation (Zusammenfassung der Merkmale des Arzneimittels) Havrix 1440. http://www.impfschaden.info/Fachinfo/Havrix-1440-GSK.pdf [zuletzt geprüft am 20.09.2014]

Haberl A: HIV und Frauen; In: HIV 2012; hrsg. v. Rockstroh JK, Hoffmann C: Medizin Fokus Verlag 2012, 490-492

Harrer T: Präventive HIV-1-Impfung: Aktueller Stand; In: HIV 2012; hrsg. v. Rockstroh JK, Hoffmann C: Medizin Fokus Verlag 2012, 45-49

Hill Z, Tawiah-Agyemang C, Odei-Danso S, Kirkwood B (2008): Informed consent in Ghana: what do participants really understand? J Med Ethics $\underline{34}$ (1), 48-53

Hillger D (2007): Chancen für alle?: Bildung zwischen Analphabetismus und Eliten-Förderung. http://www.bpb.de/internationales/asien/indien/44534/bildungssystem [zuletzt geprüft am 20.09.2014]

Hoffmann C: Antiretrovirale Therapie 2012: Substanzklassen, Medikamentenübersicht; In: HIV 2012; hrsg. v. Rockstroh JK, Hoffmann C: Medizin Fokus Verlag 2012, 64-110

Hurst SA (2008): Vulnerability in research and health care; describing the elephant in the room. Bioethics 22 (4), 191-202

Hussain S, Bharadwaj M, Nasare V, Kumari M, Sharma S (2012): Human Papillomvirus Infection Among Young Adolescents in India: Impact of Vaccination. J Med Virol $\underline{84}$ (2), 298-305

IAVI: International AIDS Vaccine Initiaitve (2013): About Us. http://www.iavi.org/Who-WeAre/Leaders/About-Us/Pages/default.aspx [zuletzt geprüft am 20.09.2014]

Indian Council of Medical Research (2006): Ethical Guidelines for Biomedical Research on Human Participants. http://icmr.nic.in/ethical_guidelines.pdf [zuletzt geprüft am 20.09.2014] Indian Council of Medical Research (2010): About ICMR. http://www.icmr.nic.in/About_Us/About_ICMR.html [zuletzt geprüft am 20.09.2014] Jacobson JL: Women's Health: The Price of Poverty; In: The health of women: A global perspective; hrsg. v. Koblinsky MA, Timyan J, Gay J: Westview Press. Boulder 1993, 3-31 Jesani A (2013): New regulations on compensation for injury and death in drug trial. Indian J Med Ethics $\underline{10}(2), 76-79$ 
Jesani A, Coutinho L (2007a): AIDS vaccine trials for India: "unanswered questions" remain unanswered. Indian J Med Ethics 4 (3), 111-112

Jesani A, Coutinho L (2007b): AIDS vaccine trials in India: ethical benchmarks and unanswered questions. Indian J Med Ethics $\underline{4}(1), 2-3$

Kang G (2010): HPV vaccines: separating real hope from drug company hype. Indian J Med Ethics $\underline{7}(1), 56-57$

Kessler M (2007): Die indischen Kennedys: Seit der Unabhängigkeit dominiert der Nehru-GandhiClan die Politik. http://www.bpb.de/internationales/asien/indien/44456/nehru-gandhi-clan [zuletzt geprüft am 20.09.2014]

Klinge I: Sex and gender in biomedicine: promises for women and men; In: Sex and gender in biomedicine: Theories, methodologies, results; hrsg. v. Wiesemann C, Klinge I: Universitätsverlag Göttingen, Göttingen 2010, 15-32

Kopelman LM: Risk and vulnerable groups; In: Encyclopedia of bioethics; hrsg. v. Post SG: 5 Bände, 3. Auflage, MacMillan Reference USA, New York 2004, 2365-2372

Kottow MH (2003): The vulnerable and the susceptible. Bioethics 17 (5-6), 460-471

Kottow MH (2004): The battering of informed consent. J Med Ethics $\underline{30}$ (6), 565-569

Kusum K: Case study: Sex selection in India; In: Women's rights and bioethics; hrsg. v. Dennerstein L, Baltes MM: UNESCO. Paris 2000, 50-58

Langlois A (2008): The UNESCO Universal Declaration on Bioethics and Human Rights: Perspectives from Kenya and South Africa. Health Care Anal 16 (1), 39-51

Levine C, Faden R, Grady C, Hammerschmidt D, Eckenwiler L, Sugarman J (2004): The Limitations of "Vulnerability" as a Protection for Human Research Participants. Am J Bioeth 4 (3), 44-49

Lidz CW, Appelbaum PS (2002): The therapeutic misconception: problems and solutions. Med Care $\underline{40}$ (9 Suppl), V55-63

Lurie P, Wolfe S (1997): Unethical Trials of Interventions to Reduce Perinatal Transmission of the human Immunodeficiency Virus in Developing Countries. N Engl J Med $\underline{337}$ (12), 853-856

Macklin R (2003): Bioethics, Vulnerability, and Protection. Bioethics 17 (5-6), 472-486

Macklin R: Research, multinational; In: Encyclopedia of bioethics; hrsg. v. Post SG: 5 Bände, 3. Auflage, MacMillan Reference USA, New York 2004, 2347-2357

Macklin R (2009): The Declaration of Helsinki: another revision. Indian J Med Ethics $\underline{6}$ (1), 2-4 
Macklin R (2010): Fair Benefits in Developing Countries: Maximin as a Good Start. Am J Bioeth 10 (6), 36-37

Macrae DJ (2007): The Council for International Organizations and Medical Sciences (CIOMS) Guidelines on Ethics of Clinical Trials. Proc Am Thorac Soc 4 (2), 176-179

Mehendale S, van Lunzen J, Clumeck N, Rockstroh J, Vets E, Johnson PR, Anklesaria P, Barin B, Boaz M, Kochhar S et al. (2008): A phase 1 study to evaluate the safety and immunogenicity of a recombinant HIV type 1 subtype $\mathrm{C}$ adeno-associated virus vaccine. AIDS Res Hum Retroviruses $\underline{24}(6), 873-880$

Mehendale S, Sahay S, Thakar M, Sahasrabuddhe S, Kakade M, Shete A, Shrotri A, Spentzou A, Tarragona T, Stevens G et al. (2010): Safety \& immunogenicity of tgAAC09, a recombinant adenoassociated virus type 2 HIV-1 subtype C vaccine in India. Indian J Med Res $\underline{132}, 168-175$ Mentschel S (2007): "Besser spät als nie": Pratibha Patil ist Indiens erste Präsidentin. http://www.bpb.de/internationales/asien/indien/44467/ehemalige-staatspraesidentin-patil [zuletzt geprüft am 20.09.2014]

Mentschel S (2012): Parteisoldat erobert Staatsspitze: Politikveteran Pranab Mukherjee ist Indiens 13. Präsident. http://www.bpb.de/internationales/asien/indien/142282/staatspraesidentmukherjee [zuletzt geprüft am 20.09.2014]

Merchant KM, Kurz KM: Women's Nutrition Through the Life Cycle: Social and Biological Vulnerabilities; In: The health of women: A global perspective; hrsg. v. Koblinsky MA, Timyan J, Gay J: Westview Press. Boulder 1993, 63-90

Ministry of Health and Family Welfare (2005): Drugs and Cosmetics (IIND Amendment) Rules: Schedule Y. http://cdsco.nic.in/Drugs\&CosmeticAct.pdf [zuletzt geprüft am 20.09.2014] Ministry of Health and Family Welfare (2013a): Drugs and Cosmetics Rules IIIND Amendment: Schedule Y. G.S.R. 72(E).

http://www.cdsco.nic.in/html/G.S.R\%2072\%28E\%29\%20dated\%2008.02.2013.pdf [zuletzt geprüft am 20.09.2014]

Ministry of Health and Family Welfare (2013b): Drugs and Cosmetics Rules IIIND Amendment: Schedule Y. G.S.R. 53(E).

http://www.cdsco.nic.in/GSR\%2053\%28E\%29\%20dated\%2030.01.2013.pdf [zuletzt geprüft am 20.09.2014] 
Ministry of Home Affairs (2001): Abstract of Speakers' Strenghth of Languages and Mother Tongues - 2001.

http://www.censusindia.gov.in/Census_Data_2001/Census_Data_Online/Language/Statement1.h tm [zuletzt geprüft am 21.09.2014]

Ministry of Home Affairs (2011a): Census Info India 2011: Final Population Totals. http://censusindia.gov.in/2011census/censusinfodashboard/index.html [zuletzt geprüft am $21.09 .2014]$

Ministry of Home Affairs (2011b): India at a glance: Census 2011. http://censusindia.gov.in/2011prov-results/indiaatglance.html [zuletzt geprüft am 21.09.2014]

Ministry of Home Affairs (2011c): Literates and literacy rates by sex. http://www.censusindia.gov.in/2011-prov-results/data_files/india/Table-2\%283\%29_literacy.pdf [zuletzt geprüft am 21.09.2014]

Ministry of Home Affairs (2011d): Provisional Population Totals, Census of India 2011: Urban Agglomerations/Cities having population 1 lakh and above. http://www.censusindia.gov.in/2011prov-results/paper2/data_files/India2/Table_3_PR_UA_Citiees_1Lakh_and_Above.pdf [zuletzt geprüft am 21.09.2014]

Ministry of Home Affairs (2011e): Provisional Population Totals Paper 1 of 2011: India Gender Composition of Population. http://www.censusindia.gov.in/2011-prov-

results/data_files/india/Final_PPT_2011_chapter5.pdf [zuletzt geprüft am 21.09.2014] Mitra AG (2013): Off-Shoring Clinical Research: Exploitation and the Reciprocity Constraint. Dev World Bioeth $\underline{13}$ (3), 111-118

National AIDS Control Organisation (2007): Women. http://naco.gov.in/NACO/Quick_Links/Women/ [zuletzt geprüft am 21.09.2014] National AIDS Control Organisation (2008): Mainstreaming HIV and AIDS for Women's Empowerment.

http://naco.gov.in/upload/Publication/IEC\%20\&\%20Mainstreaming/mainstreaming $\% 20$ hiv $\% 20$ a nd\%20aids\%20for\%20women.pdf [zuletzt geprüft am 21.09.2014]

National AIDS Control Organisation (2012): Annual Report 2011-2012.

http://www.aidsdatahub.org/dmdocuments/NACO_Annual_Report_2011_12.pdf [zuletzt geprüft am 21.09.2014]

Nadig P, Joshi M, Uthappa A (2011): Competence of ethics committees in patient protection in clinical research. Indian J Med Ethics $\underline{8}$ (3), 151-154 
Nandal S (2005): Extent and Causes of Gender and Poverty in India: A Case Study of Rural Hayana. J Int Womens Stud $\underline{7}$ (2), 182-190

NARI: National AIDS Research Institute (2010): About Us: History. http://www.nariicmr.res.in/history.php [zuletzt geprüft am 21.09.2014]

Nayyar A (2007): AIDS vaccine trials for India: getting the facts right. Indian J Med Ethics 4 (3), $109-110$

Nelson R, Merz J (2002): Voluntariness of Consent for Research. Med Care 40 (9), 69-80

Paavonen J, Naud P, Salmerón J, Wheeler CM, Chow S, Apter D, Kitchener H, Castellsague X, Teixeira JC, Skinner SR et al. (2009): Efficacy of human papillomavirus (HPV)-16/18 AS04adjuvanted vaccine against cervical infection and precancer caused by oncogenic HPV types (PATRICIA): final analysis of a double-blind, randomised study in young women. Lancet $\underline{374}$ (9689), 301-314

Pandey A, Aggarwal AR, Maulik M, Gupta J, Juneja A, Seth SD (2011): The upgraded Clinical Trials Registry India: a summary of changes. Indian J Med Ethics $\underline{8}$ (3), 186

Participantcs in the Conference on Ethical Aspects of Reserach in Developing Countries (2004): Moral Standards for Research in Developing Countries: From "Reasonable Availability" to "Fair Benefits". Hastings Cent Rep $\underline{34}$ (3), 17-27

Paul-Ehrlich-Institut (2013): Hepatitis A Impfstoffe.

http://www.pei.de/DE/arzneimittel/impfstoff-impfstoffe-fuer-den-menschen/hepatitisa/hepatitis-a-node.html [zuletzt geprüft am 21.09.2014]

Petersen B (2007): Reise durch den Selbstmordgürtel: Trotz High-Tech-Boom - Indien ist ein Agrarland. http://www.bpb.de/internationales/asien/indien/44523/agrarland-indien?p=all [zuletzt geprüft am 21.09.2014]

Phillips T (2011): Exploitation in Payments to Research Subjects. Bioethics $\underline{25}$ (4), 209-219

Pratt B, Lwin KM, Zion D, Nosten F, Loff B, Cheah PY (2013): Exploitation and community engagement: Can Community Advisory Boards successfully assume a role minimising exploitation in international research? Dev World Bioeth, 1-9

Qadeer I: Public health in India: Critical reflections (Critical public health series 1); Daanish Books, Delhi 2011

Resnik D (2004): Research Subjects in Developing Nations and Vulnerability. Am J Bioeth 4 (3), 6364 
Rid A, Schmidt H (2009): Die erneut überarbeitete Deklaration von Helsinki: Wie sind die Änderungen aus ethischer Sicht zu beurteilen? Dtsch med Wochenschr 134 (49), 2525-2528 Robert Koch-Institut, Gesellschaft der epidemiologischen Krebsregister in Deutschland e. V. (2012): Krebs in Deutschland 2007/2008.

http://www.krebsdaten.de/Krebs/DE/Content/Publikationen/Krebs_in_Deutschland/kid_2012 /krebs_in_deutschland_2012.pdf?_blob=publicationFile [zuletzt geprüft am 21.09.2014]

Rocca CH, Rathod S, Falle T, Pande Rohini P, Krishnan S (2009): Challenging assumptions about women's empowerment: social and economic resources and domestic violence among young married women in urban South India. Int J Epidemiol $\underline{38}$ (2), 577-585

Rockstroh JK: Einleitung; In: HIV 2012; hrsg. v. Rockstroh JK, Hoffmann C: Medizin Fokus Verlag 2012, 2-14

Rubbert-Roth A, Behrens G: Pathophysiologie der HIV-Infektion; In: HIV 2012; hrsg. v. Rockstroh JK, Hoffmann C: Medizin Fokus Verlag 2012, 22-44

Sahay S, Mehendale S (2004): Addressing ethical concerns in the Indian HIV vaccine trials. Indian J Med Ethics 1 (4), 109-112

Sama Resource Group for Women and Health. http://www.samawomenshealth.org/index.html [zuletzt geprüft am 21.09.2014]

Sama Resource Group for Women and Health (2010): Concerns about the human papillom virus (HPV) vaccine. Indian J Med Ethics $\underline{7}, 38-41$

Schicktanz S (2009): Zum Stellenwert von Betroffenheit, Öffentlichkeit und Deliberation im empirical turn der Medizinethik. Ethik in der Medizin 21 (3), 223-234

Schiffmann M, Castle PE, Jeronimo J, Rodriguez AC, Wacholder S (2007): Human papillomavirus and cervical cancer. Lancet $\underline{370}$ (9590), 890-907

Schöne-Seifert B: Medizinethik; In: Angewandte Ethik: Die Bereichsethiken und ihre theoretische Fundierung. Ein Handbuch; hrsg. v. Nida-Rümelin J, 2., aktualisierte Auflage, Alfred Kröner, Stuttgart 2005, 691-792

Sengupta A (2009): Fatal trials: clinical trials are killing people. Indian J Med Ethics $\underline{6}$ (3), 118-119 Sharma BR, Gupta M (2004): Gender Based Violence in India - A Never-ending Phenomenon. J Int Womens Stud $\underline{6}(1), 114-123$

Shastri S (2010): Cervical cancer sreening and vaccination in India. Indian J Med Ethics $\underline{7}$ (1), 41-42 Singh JA (2013): India's regulatory reforms on compensation for clinical trial injuries and deaths: urgent need for revisiting. Indian J Med Ethics 10 (3), 195-197 
Singh K: Obstacles to Women's Rights in India; In: Human rights of women: National and international perspectives; hrsg. v. Cook RJ: University of Pennsylvania Press, Philadelphia 1995, 375-395

Skoda U (2007): Kaste und Kastensystem in Indien: Eine Einführung.

http://www.bpb.de/internationales/asien/indien/44414/kastenwesen?p=all [zuletzt geprüft am 21.09.2014]

Srinivasan S (2005): Daughter or Dowries? The Changing Nature of Dowry Practice in South India. World Development $\underline{33}$ (4), 593-615

Srinivasan S (2007): Domestic Violence and Dowry: Evidence from a South Indian Village. World Development $\underline{35}(5), 857-880$

Srinivasan S (2009): Trial by fire. http://infochangeindia.org/public-health/analysis/trial-byfire.html [zuletzt geprüft am 21.09.2014]

Srinivasan S (2011): HPV vaccine trials and sleeping watchdogs. Indian J Med Ethics $\underline{8}$ (2), 73-74

Ständige Impfkommission am Robert Koch-Institut (2007): Impfung gegen humane Papillomaviren (HPV) für Mädchen von 12 bis 17 Jahren - Empfehlung und Begründung. Epidemiologisches Bulletin, 97-103

Ständige Impfkommission am Robert Koch-Institut (2009): Impfung gegen HPV - Aktuelle Bewertung der STIKO. Epidemiologisches Bulletin, 319-328

Ständige Impfkommission am Robert Koch-Institut (2012): Empfehlungen der Ständigen Impfkommission (STIKO) am Robert Koch-Institut/Stand: Juli 2012. Epidemiologisches Bulletin, 284-310

Suhadev M, Nyamathi AM, Swaminathan S, Venkatesan P, Raja Sakthivel M, Shenbagavalli R, Suresh A, Fahey JL (2006): A pilot study on willingness to participate in future preventive HIV vaccine trials. Indian J Med Res 124 (6), 631-640

Taur SR, Bavdekar SB, Thatte UM (2011): Survey of ethics committee protocol approval letters: compliance with Schedule Y/ICMR Guidelines 2006. Indian J Med Ethics $\underline{8}$ (4), 214-216 Tharyan P (2007): Ethics committees and clinical trials registration in India: opportunities, obligations, challenges and solutions. Indian J Med Ethics $\underline{4}$ (4), 168-169

The World Bank (2013a): Data: Births attended by skilled health staff (\% of total). http://data.worldbank.org/indicator/SH.STA.BRTC.ZS/countries [zuletzt geprüft am 21.09.2014] 
The World Bank (2013b): Data: Children in employment, total (\% of children ages 7-14). http://data.worldbank.org/indicator/sl.tlf.0714.zs/countries?page=1 [zuletzt geprüft am 21.09.2014]

The World Bank (2013c): Data: Children out of school, primary, female. http://data.worldbank.org/indicator/SE.PRM.UNER.FE/countries [zuletzt geprüft am 21.09.2014]

The World Bank (2013d): Data: Children out of school, primary, male. http://data.worldbank.org/indicator/SE.PRM.UNER.MA/countries [zuletzt geprüft am 21.09.2014]

The World Bank (2013e): Data: Employment in agriculture (\% of total employment). http://data.worldbank.org/indicator/SL.AGR.EMPL.ZS [zuletzt geprüft am 21.09.2014] The World Bank (2013f): Data: GDP growth (annual \%). http://data.worldbank.org/indicator/NY.GDP.MKTP.KD.ZG [zuletzt geprüft am 20.01.2014] The World Bank (2013g): Data: Gross intake ratio in first grade of primary education, female (\% of relevant age group). http://data.worldbank.org/indicator/SE.PRM.GINT.FE.ZS/countries [zuletzt geprüft am 21.09.2014]

The World Bank (2013h): Data: Gross intake ratio in first grade of primary education, male (\% of relevant age group). http://data.worldbank.org/indicator/SE.PRM.GINT.MA.ZS/countries [zuletzt geprüft am 21.09.2014]

The World Bank (2013i): Data: Health expenditure, total (\% of GDP). http://data.worldbank.org/indicator/SH.XPD.TOTL.ZS/countries [zuletzt geprüft am 21.09.2014]

The World Bank (2013)): Data: Immunization, DPT (\% of children ages 12-23 months). http://data.worldbank.org/indicator/SH.IMM.IDPT/countries [zuletzt geprüft am 21.09.2014] The World Bank (2013k): Data: Improved sanitation facilities (\% of population with access). http://data.worldbank.org/indicator/SH.STA.ACSN/countries [zuletzt geprüft am 21.09.2014] The World Bank (20131): Data: Life expectancy at birth, female (years). http://data.worldbank.org/indicator/SP.DYN.LE00.FE.IN/countries [zuletzt geprüft am 21.09.2014]

The World Bank (2013m): Data: Life expectancy at birth, male (years). http://data.worldbank.org/indicator/SP.DYN.LE00.MA.IN/countries [zuletzt geprüft am 21.09.2014] 
The World Bank (2013n): Data: Life expectancy at birth, total (years).

http://data.worldbank.org/indicator/SP.DYN.LE00.IN [zuletzt geprüft am 21.09.2014]

The World Bank (2013o): Data: Literacy rate, adult total (\% of people ages 15 and above).

http://data.worldbank.org/indicator/SE.ADT.LITR.ZS/countries [zuletzt geprüft am 21.09.2014]

The World Bank (2013p): Data: Maternal mortality ratio (modeled estimate, per 100,000 live births). http://data.worldbank.org/indicator/SH.STA.MMRT/countries [zuletzt geprüft am 21.09.2014]

The World Bank (2013q): Data: Mortality rate, under-5 (per 1,000 live births).

http://data.worldbank.org/indicator/SH.DYN.MORT/countries [zuletzt geprüft am 21.09.2014]

The World Bank (2013r): Data: Population ages 0-14 (\% of total).

http://data.worldbank.org/indicator/SP.POP.0014.TO.ZS/countries [zuletzt geprüft am 21.09.2014]

The World Bank (2013s): Data: Poverty headcount ratio at $\$ 1.25$ a day (PPP) ( $\%$ of population). http://data.worldbank.org/indicator/SI.POV.DDAY [zuletzt geprüft am 21.09.2014]

The World Bank (2013t): Data: Pregnant women receiving prenatal care (\%).

http://data.worldbank.org/indicator/SH.STA.ANVC.ZS/countries [zuletzt geprüft am $21.09 .2014]$

The World Bank (2013u): Data: Prevalence of undernourishment (\% of population). http://data.worldbank.org/indicator/SN.ITK.DEFC.ZS [zuletzt geprüft am 21.09.2014]

The World Bank (2013v): Data: Proportion of seats held by women in national parliaments (\%). http://data.worldbank.org/indicator/SG.GEN.PARL.ZS/countries [zuletzt geprüft am 21.09.2014]

The World Bank (2013w): Data: Ratio of female to male secondary enrollment (\%). http://data.worldbank.org/indicator/SE.ENR.SECO.FM.ZS/countries [zuletzt geprüft am $21.09 .2014]$

The World Bank (2013x): Data: Ratio of female to male tertiary enrollment (\%). http://data.worldbank.org/indicator/SE.ENR.TERT.FM.ZS/countries [zuletzt geprüft am 21.09.2014]

The World Bank (2013y): Data: School enrollment, secondary (\% gross). http://data.worldbank.org/indicator/SE.SEC.ENRR/countries [zuletzt geprüft am 21.09.2014] The World Bank (2013z): Data: School enrollment, tertiary (\% gross). http://data.worldbank.org/indicator/SE.TER.ENRR/countries [zuletzt geprüft am 21.09.2014] 
Thomas J (2004): Unmet ethical concerns of the proposed preventive HIV vaccine trials in India. Indian J Med Ethics 1 (3), 87-88

UNAIDS: AIDS Info: Epidemiological Status. http://www.unaids.org/en/dataanalysis/datatools/aidsinfo/ [zuletzt geprüft am 21.09.2014] UNAIDS (2008): Fast facts about HIV.

http://www.unaids.org/en/media/unaids/contentassets/dataimport/pub/factsheet/2008/200805 19_fastfacts_hiv_en.pdf [zuletzt geprüft am 21.09.2014]

UNAIDS (2009): India: Country Situation. http://www.unaids.org/ctrysa/ASIIND_en.pdf [zuletzt geprüft am 21.09.2014]

UNAIDS (2010): Global Report: UNAIDS Report on the Global AIDS Epidemic. http://www.unaids.org/globalreport/documents/20101123_GlobalReport_full_en.pdf [zuletzt geprüft am 21.09.2014]

UNAIDS (2013): Global Report: UNAIDS Report on the Global AIDS Epidemic. http://www.unaids.org/en/media/unaids/contentassets/documents/epidemiology/2013/gr2013/ UNAIDS_Global_Report_2013_en.pdf [zuletzt geprüft am 21.09.2014]

UNAIDS (2012a): India: HIV and AIDS estimates (2012). http://www.unaids.org/en/regionscountries/countries/india/ [zuletzt geprüft am 21.09.2014] UNAIDS (2012b): Global AIDS Epidemic facts and figures. http://www.unaids.org/en/media/unaids/contentassets/documents/epidemiology/2012/gr2012/ 20121120_FactSheet_Global_en.pdf [zuletzt geprüft am 21.09.2014]

UNESCO: Über die UNESCO. http://www.unesco.de/ueber_die_unesco.html [zuletzt geprüft am 21.09.2014]

UNESCO: Allgemeine Erklärung über Bioethik und Menschenrechte, Paris 2005a

UNESCO: Universal Declaration on Bioethics and Human Rights, Paris 2005b

Unesco Institute for Statistics (2011): Universal Primary Education. http://www.uis.unesco.org/FactSheets/Documents/fs8-2011-en.pdf [zuletzt geprüft am 21.09.2014]

UNGASS: United Nations General Assembly Special Session (2010): Country Progress Report India.

http://www.unaids.org/en/dataanalysis/knowyourresponse/countryprogressreports/2010countrie s/india_2010_country_progress_report_en.pdf [zuletzt geprüft am 21.09.2014] 
United Nations Development Programme: Human Development Index (HDI). http://hdr.undp.org/en/statistics/hdi/ [zuletzt geprüft am 21.09.2014]

United Nations Development Programme (2013): Human Development Index (HDI) - 2012 Rankings. http://hdr.undp.org/en/statistics/ [zuletzt geprüft am 21.09.2014]

United Nations Statistics Division (2010a): The World's Women 2010: Trends and Statistics: Poverty. http://unstats.un.org/unsd/demographic/products/Worldswomen/wwPov2010.htm [zuletzt geprüft am 21.09.2014]

United Nations Statistics Division (2010b): The World's Women 2010: Trends and Statistics: Health. http://unstats.un.org/unsd/demographic/products/Worldswomen/wwhealth2010.htm [zuletzt geprüft am 21.09.2014]

United Nations Statistics Division (2010c): The World's Women 2010: Trends and Statistics: Violence against women.

http://unstats.un.org/unsd/demographic/products/Worldswomen/wwVaw2010.htm [zuletzt geprüft am 21.09.2014]

Vardas E, Kaleebu P, Bekker L, Hoosen A, Chomba E, Johnson PR, Anklesaria P, Birungi J, Barin B, Boaz M et al. (2010): A phase 2 study to evaluate the safety and immunogenicity of a recombinant HIV type 1 vaccine based on adeno-associated virus. AIDS Res Hum Retroviruses $\underline{26}$ (8), 933-942

Wertheimer A: Exploitation; Princeton University Press, Princeton, N.J 1996

Wertheimer A, Millum J, Schaefer GO (2010): Why Adopt a Maximin Theory of Exploitation? Am J Bioeth $\underline{10}$ (6), 38-39

Wessler HW (2007): Indien - Eine Einführung: Herausforderungen für die aufstrebende asiatische Großmacht im 21. Jahrhundert. http://www.bpb.de/internationales/asien/indien/44581/indieneine-einfuehrung?p=all [zuletzt geprüft am 21.09.2014]

WHO (2014): Glossary. http://www.who.int/ictrp/glossary/en/index.html\#TrialPhase [zuletzt geprüft am 21.09.2014]

Wild V (2007): Plädoyer für einen Einschluss schwangerer Frauen in Arzneimittelstudien. Ethik in der Medizin $\underline{19}$ (1), 7-23

World Medical Association: Declaration of Helsinki: Ethical Principles for Medical Research Involving Human Subjects, Seoul 2008

World Medical Association: Declaration of Helsinki: Ethical Principles for Medical Research Involving Human Subjects, Fortaleza 2013a 
World Medical Association (2013b): About the WMA.

http://www.wma.net/en/60about/index.html [zuletzt geprüft am 21.09.2014]

World Medical Association (2013c): Handbook of Declarations.

http://www.wma.net/en/30publications/10policies/10about/ [zuletzt geprüft am 21.09.2014] 


\section{Abkürzungsverzeichnis}

$\mathrm{AE} \quad$ adverse events

AIDS

ART

ATP

BUKO

CDSCO

CIN

CIOMS

CIS

CONSORT

$\mathrm{CRO}$

CTRI

DCGI

DHR

ELISA

ELISPOT

FUTURE

GSK

HDI

HIV

$\mathrm{HPV}$

IAVI

IBC

ICMR

IDU

IGBC

MOHFW

MSC

MSM acquired immunodeficiency syndrome

Antiretrovirale Therapie

according to protocol

Bundeskoordination Internationalismus

Central Drug Standard Control Organization

cervical intraepithelial neoplasia

Council for International Organizations of Medical Sciences

carcinoma in situ

Consolidated Standards of Reporting Trials

Clinical Research Organizations

Clinical Trials Registry India

Drugs Controller General of India

Department of Health Research

Enzyme-linked Immunosorbent Assays

IFN- $\gamma$ secretory Enzyme Linked Immuno Spot Assay

Females United to Unilaterally Reduce Endo/Ectocervical Disease

GlaxoSmithKline

buman development index

humanes Immundefizienz-Virus

humane Papillom-Viren

International AIDS Vaccine Initiative

International Bioethics Committee

Indian Council of Medical Research

intravenous drug users

Intergovernmental Bioethics Committee

Ministry of Health and Family Welfare

medically significant conditions

men who have sex with men 
NACO

NACP

NARI

NOCD

PATRICIA

SAE

STIKO

TVC

UNESCO

UNGASS

WHO

WMA
National AIDS Control Organisation

National AIDS Control Programm

National AIDS Research Institute

new-onset cbronical disorder

PApilloma TRIal against Cancer In young Adults

serious adverse events

Ständige Impfkommission am Robert Koch-Institut

total vaccinated cohort

United Nations Educational, Scientific and Cultural Organization

United Nations General Assembly Special Session

World Health Organization

World Medical Association 


\section{$\underline{\text { Lebenslauf }}$}

Ich, Helen Grete Orth, wurde am 15. Oktober 1985 als zweites Kind meiner Eltern Andrea Orth (geb. Burkart) und Hermann Orth in Neustadt an der Weinstraße geboren.

Ab 1995 besuchte ich das Leibniz Gymnasium in Neustadt an der Weinstraße. 2001 verbrachte ich sechs Monate bei einer Gastfamilie in Nashville, USA und besuchte dort die Beech High School. Im März 2005 schloss ich meine Schulausbildung mit der Allgemeinen Hochschulreife, einschließlich eines Zertifikates über die bilinguale (deutsch-französisch) mündliche Abiturprüfung in Gemeinschaftskunde, ab.

Nach der Schulzeit lebte ich ein Jahr in Cochabamba, Bolivien, und arbeitete dort als Freiwillige im Centro Cultural Ayopaymanta und im Straßenkinderheim Madre de Dios.

Zum Sommersemester 2007 begann ich das Studium der Humanmedizin an der Georg-August Universität in Göttingen. Das Studium schloss ich im Juni 2014 mit dem 2. Abschnitt der Ärztlichen Prüfung erfolgreich ab.

Nach Absolvierung der 1. Prüfung des Ärztlichen Abschnitts begann ich im Herbst 2010 mit ersten Recherchen für die vorliegende Dissertation mit dem Thema „Das Konzept der Vulnerabilität im Kontext transnationaler Biomedizin: Eine ethische Analyse am Beispiel von Forschung mit Frauen in Indien“ unter der Leitung von Frau Prof. Dr. Silke Schicktanz. 


\section{Danksagung}

Besonders danken möchte ich hiermit meiner Betreuerin, Frau Prof. Dr. Silke Schicktanz. Danken möchte ich ihr für die Inspiration, eine Doktorarbeit im medizinethischen Bereich zu schreiben, durch ein von ihr gehaltenes Seminar zu Bioethikrichtlinien im ersten klinischen Semester. Außerdem bin ich ihr nicht nur für das spannende Thema, sondern vor allem auch für die hervorragende Betreuung sehr dankbar.

Nicht unerwähnt lassen möchte ich auch Sabine Wöhlke, der ich sowohl für inhaltliche Diskussionen als auch für ihre persönliche Unterstützung dankbar bin.

Großer Dank gebührt zudem Torsten Kellner, Lotta König und Lea Fischer für inhaltliche Anregungen, für die Hilfe beim Korrekturlesen, insbesondere jedoch für das an meiner Seite sein während aller Höhen und Tiefen des Promovierens. Herzlichster Dank gilt zudem meiner Familie, ohne deren Unterstützung diese Arbeit nicht möglich gewesen wäre. 